Karsten J. Adamski

\title{
Mikroanalyse eines Grundrentenkonzeptes der Alterssicherung
}

Eine empirische Studie zur Wirkung einer Grundrente auf Basis des Sozioökonomischen Panels 


\section{Mikroanalyse eines Grundrentenkonzeptes der Alterssicherung}

Die konzeptionelle Ausgestaltung der Alterssicherung, das heißt die Gratwanderung zwischen Bismarck und Beveridge, wird wegen der sich abzeichnenden demografischen Veränderungen kontrovers diskutiert. Eine besondere Bedeutung erlangte hierbei die Idee einer Bürgerrente, in der pro Kopf einheitliche Leistungen mit lohnproportionalen Beiträgen verknüpft werden. Die mit einer Bürgerrente einhergehenden Kostenwirkungen und Einkommensveränderungen für Altersrentner werden quantitativ untersucht. In einer Erweiterung des Standardrentnerkonzeptes erfolgt die Analyse auf Grundlage von empirisch ermittelten Erwerbsbiografien, die für eine bestimmte Kohorte typisch sind. Hierdurch können die Folgen einer Systemumstellung auch für Personen mit unstetigen Lebensläufen abgebildet werden.

Karsten J. Adamski wurde 1973 in Albersdorf (Dithmarschen) geboren. Er studierte Betriebswirtschaftslehre an der Universität der Bundeswehr Hamburg sowie an der Arizona State University und wurde 1997 zum Berufssoldaten ernannt. Von 2001 bis 2004 war er als Wissenschaftlicher Mitarbeiter am Institut für Statistik und quantitative Ökonomik an der Universität der Bundeswehr tätig. Hierbei war er insbesondere für die Bearbeitung eines gemeinschaftlichen Forschungsprojektes des Freistaates Sachsen und des Hamburgischen Welt-Wirtschafts-Archivs (HWWA) und der Universität der Bundeswehr Hamburg verantwortlich. Die Promotion erfolgte 2004. 
Mikroanalyse eines Grundrentenkonzeptes der Alterssicherung 


\section{SCHRIFTEN ZUR \\ WIRTSCHAFTSTHEORIE UND WIRTSCHAFTSPOLITIK}

Herausgegeben von

Rolf Hasse, Jörn Kruse, Wolf Schäfer, Thomas Straubhaar und Klaus W. Zimmermann

Band 31

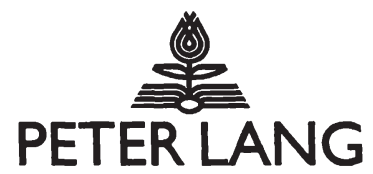

Frankfurt am Main - Berlin - Bern - Bruxelles - New York - Oxford - Wien 
Karsten J. Adamski

\section{Mikroanalyse eines Grundrentenkonzeptes derAlterssicherung}

Eine empirische Studie zurWirkung einer Grundrente auf Basis des

Sozioökonomischen Panels

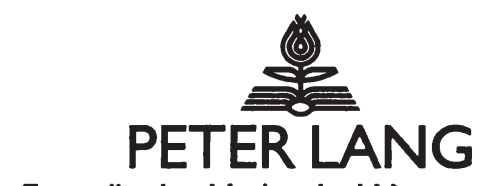

EuropäischerVerlag derWissenschaften 
Bibliografische Information Der Deutschen Bibliothek

Die Deutsche Bibliothek verzeichnet diese Publikation in der Deutschen Nationalbibliografie; detaillierte bibliografische Daten sind im Internet uber <http://dnb.ddb.de> abrufbar.

Zugl.: Hamburg, Univ. der Bundeswehr, Diss., 2004 Open Access: The online version of this publication is published on www.peterlang.com and www.econstor.eu under the international Creative Commons License CC-BY 4.0. Learn more on how you can use and share this work: http://creativecommons.org/licenses/ by/4.0.

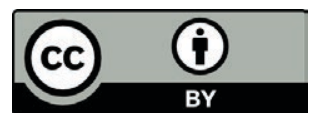

This book is available Open Access thanks to the kind support of ZBW - Leibniz-Informationszentrum Wirtschaft.

Gedruckt mit Unterstützung der Helmut-Schmidt-Universität, Universităt der Bundeswehr Hamburg.

Gedruckt auf alterungsbeständigem, săurefreiem Papier.

D705

ISSN $1433-1519$

ISBN3-631-52662-8

ISBN 978-3-631-75003-2 (eBook)

(C) Peter Lang GmbH

Europåischer Verlag der Wissenschaften

Frankfurt am Main 2004

Alle Rechte vorbehalten.

Das Werk einschließlich aller seiner Teile ist urheberrechtlich geschützt. Jede Verwertung außerhalb der engen Grenzen des Urheberrechtsgesetzes ist ohne Zustimmung des Verlages unzulăssig und strafbar. Das gilt insbesondere furr Vervielfaltigungen, Übersetzungen, Mikroverfilmungen und die Einspeicherung und Verarbeitung in elektronischen Systemen.

Printed in Germany 124567

www.peterlang.de 


\section{Unseren Zwillingen}


Karsten J. Adamski - 978-3-631-75003-2

Downloaded from PubFactory at 01/11/2019 09:24:00AM

via free access 


\section{Vorwort}

Die vorliegende Arbeit entstand während meiner dreijährigen Tätigkeit als wissenschaftlicher Mitarbeiter am Institut für Statistik und quantitative Ökonomik der Helmut-Schmidt-Universität, Universität der Bundeswehr Hamburg. Sie ist das Ergebnis eines gemeinschaftlichen Forschungsvorhabens des Freistaates Sachsen, des Hamburgischen Welt-Wirtschafts-Archivs und der Helmut-Schmidt-Universität, Universität der Bundeswehr Hamburg: „Umstellung des Rentensystems auf eine Grundrente".

Ich möchte mich insbesondere bei meinem akademischen Lehrer und Doktorvater Prof. Dr. Götz Uebe für die Betreuung und Unterstützung bedanken. Nur aufgrund seines persönlichen Engagements und seiner Fürsprache ist dieses Projekt ermöglicht worden. Zudem stand er mir während der gesamten Bearbeitungszeit stets zur Seite und hat mit unzähligen Anregungen die Arbeit maßgeblich geprägt.

Bedanken möchte ich mich auch bei Prof. Dr. Thomas Straubhaar für die persönliche Betreuung, die Übernahme der Begutachtung und die zahlreichen Anregungen. Erst durch seinen persönlichen Einsatz und durch die Mitförderung aus den Mitteln der Forschungsförderung des Hamburgischen WeltWirtschafts-Archivs konnte das Forschungsvorhaben begründet werden.

Einen ganz herzlichen Dank möchte ich der Staatskanzlei des Freistaates Sachsen aussprechen. Trotz einer allgemein knappen Haushaltslage hat sich der Freistaat entschieden, ein wissenschaftliches Projekt zur Neuordnung der Alterssicherungssysteme zu unterstützen. Ganz besonders hervorheben möchte ich hierbei den persönlichen Einsatz des Alt-Ministerpräsidenten, Herrn Prof. Dr. Kurt Biedenkopf. Mein Dank gilt auch dem fortwährend gezeigten Interesse und der stets freundlichen Betreuung durch Frau Dr. Jenschke.

Danken möchte ich auch der Helmut-Schmidt-Universität, Universität der Bundeswehr Hamburg für die Möglichkeit, als wissenschaftlicher Mitarbeiter am Institut von Prof. Dr. Götz Uebe tätig werden zu können. Zudem bedanke ich mich für die finanzielle Mitförderung des Vorhabens. Der Dank richtet sich insbesondere an den Kanzler der Helmut-Schmidt-Universität, Universität der Bundeswehr Hamburg, Herrn Eckhard Redlich.

Einen ebenfalls ganz besonderen Dank möchte ich an die Personalführung der Bundeswehr richten. Nur infolge der gewährten Freistellung vom aktiven militärischen Dienst war es mir als Berufssoldat möglich, das Projekt zu bearbeiten und meine Promotion zu betreiben. 
Ein besonderer Dank gilt auch der Hamburgischen Landesbank und der Gesellschaft der Freunde und Förderer der Universität der Bundeswehr Hamburg e.V. für die finanzielle Unterstützung, durch die ich an wissenschaftlichen Tagungen und Seminaren teilnehmen konnte.

Ebenso möchte ich mich bei meinen Kollegen Dr. Martin Schäfer und Dipl. Math. Frank Kubitschek für die stets geleistete Hilfe, Unterstützung und für lange, intensive Diskussionen bedanken, die den Inhalt der Arbeit maßgeblich geprägt haben.

Für das mir entgegengebrachte Vertrauen und die finanzielle Förderung des Forschungsvorhabens gilt allen ein ganz herzlicher Dank.

$\mathrm{Zu}$ guter Letzt möchte ich mich auch bei meiner Familie bedanken, die mich auf vielfältige Art und Weise unterstützt hat und die oftmals über die aktuellen Probleme der Rentensysteme diskutieren musste. Hervorzuheben ist insbesondere die Unterstützung durch meine Frau Michaela.

Karsten J. Adamski

Hamburg, im November 2003 


\section{Index}

Vorwort 7

0. Einführung und Überblick 13

1. Die Bürgerrente nach Biedenkopf..............................................19

1.1 Die Grundgedanken .......................................................................19

1.2 Leistungen und Anspruchsvoraussetzungen ........................................20

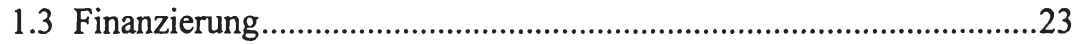

1.4 Der Systemübergang ...........................................................................24

1.5 Zusammenfassung..................................................................................25

\section{Grundtypen der Alterssicherung - Allgemeine Aussagen}

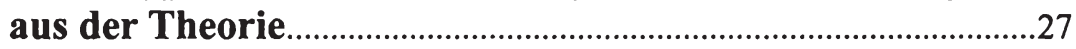

2.1 Umlage- versus Kapitaldeckungsverfahren ........................................27

2.2 Möglichkeiten eines pareto-effizienten Systemübergangs....................30

2.3 Zusammenfassung ...............................................................................

3. Die Kosten einer Bürgerrente ……………………........................35

3.1 Vorhandene Berechnungen - Ein Literaturüberblick.............................35

3.1.1 Vorbemerkungen ....................................................................35

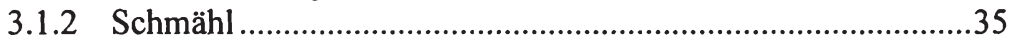

3.1.3 Buttler, Jäger, Rappl.............................................................36

3.1.4 Müller, Tautz …………………………………………….....37

3.1.5 Besendorfer, Borgmann, Raffelhüschen ...................................40

3.1.6 Burgert, Dicke, Glismann, Horn ...............................................41

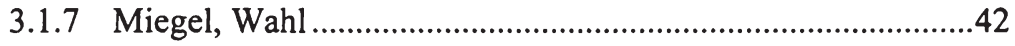

3.1.8 Zusammenfassung ..............................................................44

3.1.8.1 Wesentliche Ergebnisse der Modellrechnungen ........44

3.1.8.2 Implikationen der Modellgestaltung ............................45

3.2 Kosten einer Bürgerrente: Eine neue Berechnung auf der Grundlage von Mikrodaten ...........................................................................52

3.2.1 Vorbemerkungen ...................................................................52

3.2.2 Die Modelldarstellung ........................................................53

3.2.2.1 Bürgerrente wegen Alters ........................................55

3.2.2.2 Bürgerrente wegen Todes ...........................................55

3.2.2.3 Bürgerrente wegen einer Erwerbsminderung.............56

3.2.2.4 Die Leistungshöhe ........................................................56 
3.2.2.5 Das lebenslauf- und altersbezogene Territorialprinzip....................................................................... 58

3.2.3 Ein Kostenvergleich: Bestehende Systeme und Bürgerrente . 60

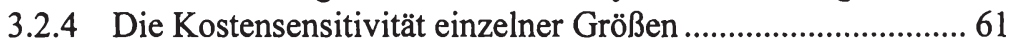

3.2.4.1 Die Regelaltersgrenze .............................................. 62

3.2.4.2 Die Einschränkungen bei der Hinterbliebenen-

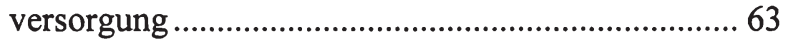

3.2.4.3 Das lebenslauf- und altersbezogene Territorial-

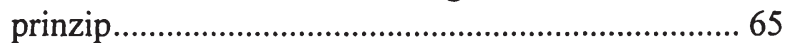

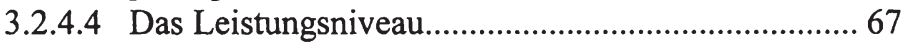

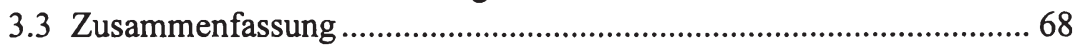

4. Die Einkommen der Altersrentner.......................................... 71

4.1 Die Identifikation repräsentativer Erwerbsbiographien .................... 72

4.1.1 Ermittlung individueller Tätigkeitsprofile .............................. 72

4.1.2 Optimal Matching Analysis - Eine Einführung ....................... 74

4.1.3 Ermittlung typischer Verlaufsmuster .................................... 78

4.1.3.1 Gruppierung der individuellen Tätigkeitsprofile...... 78

4.1.3.2 Reduktion auf einen typischen Verlauf .................... 81

4.1.4 Darstellung ausgewählter Ergebnisse ..................................... 84

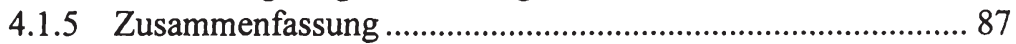

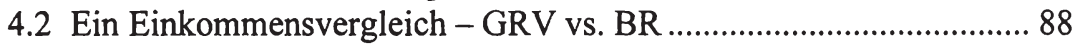

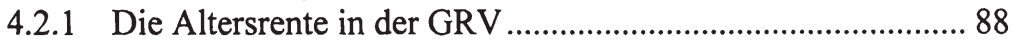

4.2.2 Die Einkommen der Altersrentner in einer Bürgerrente ........ 94

4.2.2.1 Die Beitragssätze in einem Grundrentensystem....... 95

4.2.2.2 Die vollständige Erwerbsbiographie ........................ 97

4.2.2.3 Die unvollständige Erwerbsbiographie .................. 100

4.2.2.4 Abweichen von einer Normalerwerbsbiographie... 105

4.2.3 Untersuchungsergebnisse .................................................. 111

4.2.3.1 Analyse der typischen Personen ............................. 111

4.2.3.2 Gewinner und Verlierer eines Systemwechsels ..... 121

4.2.4 Umverteilungen in den Rentensystemen ............................. 129

4.2.4.1 Intragenerationale Umverteilungen ....................... 130

4.2.4.2 Intergenerationale Umverteilungen ....................... 132

4.2.5 Zusammenfassung ........................................................... 136 
5. Kostenprojektion und Überführung der Rentensysteme ...139

5.1 Annahmen zur Projektion: Ökonomische und demographische Entwicklung bis 2040 ..................................................................140

5.2 Kostenentwicklung einer eingeführten Bürgerrente - Eine Vorausberechnung bis zum Jahr 2040....................................................142

5.3 Zusammenfassung .........................................................................147

5.4 Die Modellierung eines Systemübergangs..........................................148

5.4.1 Der Modellaufbau.................................................................149

5.4.1.1 Die Umstellung der gesetzlichen Rentenversicherung ............................................................149

5.4.1.2 Die Überführung der sonstigen Systeme...................153

5.4.2 Die Mehrbelastung im Übergang .............................................155

5.5 Zusammenfassung .............................................................................159

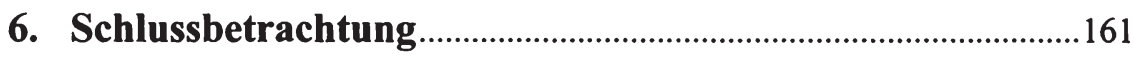

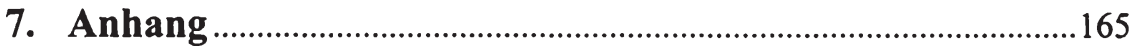

7.1 Tabellen und Abbildungen................................................................165

7.2 Überblick über ausgewählte Algorithmen .........................................171

7.4 Abkürzungsverzeichnis ..................................................................180

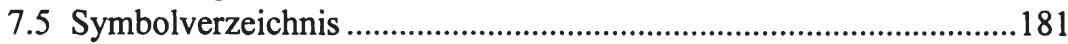

7.6 Tabellen- und Abbildungsverzeichnis..................................................185

7.7 Literaturverzeichnis......................................................................... 188 
Karsten J. Adamski - 978-3-631-75003-2

Downloaded from PubFactory at 01/11/2019 09:24:00AM

via free access 


\section{0. $\quad$ Einführung und Überblick}

Die demographischen Veränderungen bedeuten eine nachhaltige Belastungsprobe für die Finanzierbarkeit der bestehenden Alterssicherungssysteme. Dies ist lange bekannt und vielfach erörtert worden. Wie jüngst die „Kommission für die Nachhaltigkeit in der Finanzierung der Sozialen Sicherungssysteme“" („Rürup-Kommission“) und die Kommission „Soziale Sicherheit“ („HerzogKommission") erinnerten, ${ }^{1}$ wirken eine steigende Lebenserwartung in Kombination mit einer sinkenden Fertilitätsrate und mäßigen Wanderungsgewinnen unmittelbar auf die Bevölkerungsstruktur. Die Bevölkerung altert beträchtlich. Beträgt derzeit das Verhältnis der Personen in einem Lebensalter von 65 oder mehr Jahren zu den 20- bis 64-Jährigen rund 26,7 v.H. ${ }^{2}$ sind im Jahr 2040 zwischen 51 v.H. und 55 v.H. zu erwarten. ${ }^{3}$ Als unmittelbare Folge dieser Veränderungen steigen die Kosten der Sozialsysteme erheblich. Beispielsweise wird der Beitragssatz zur gesetzlichen Rentenversicherung von derzeit 19,3 v.H. bis zum Jahr 2030 auf rund 23,3 v.H. bis 25,9 v.H. ansteigen. ${ }^{4}$ Eine Fortführung des bestehenden Generationenvertrages führt folglich zu erheblichen Mehrbelastungen der Erwerbstätigen und zu einer Steigerung der Lohnnebenkosten.

Um eine finanzielle Tragfähigkeit und damit eine Stabilität und Verlässlichkeit der Sozialsysteme auch in Zukunft gewährleisten zu können, sind Veränderungen unausweichlich. Wenngleich die diskutierten Lösungsansätze im Detail signifikante Unterschiede aufweisen, zeigt die Neuordnung des Gesundheitswesens ${ }^{5}$ die grundsätzliche Zielvorstellung der aktuellen Reformbestrebungen in der Sozialpolitik auf:

1 Vgl. Kommission für die Nachhaltigkeit in der Finanzierung der Sozialen Sicherungssysteme (2003b), Kommission Soziale Sicherheit (2003).

Mit Stand vom Jahr 2000. Vgl. Statistisches Bundesamt (lfd. Jahre a).

Für Wanderungsgewinne in Höhe von 200000 Personen p.a. errechnet die Rürup-Kommission (2003b, S. 54f.) für das Jahr 2040 einen Altenquotienten in Höhe von 52,6 v.H. Die Herzog-Kommission (2003, S. 9) erwartet hingegen einen durchschnittlichen Wanderungssaldo in Höhe von 100000 Personen p.a., so dass der Altenquotient entsprechend höher ausfällt. $\mathrm{Zu}$ ähnlichen Ergebnissen gelangen auch (angegeben sind nur die Ergebnisse für Wanderungssalden in Höhe von 100000 Personen p.a.): Prognos (1998): 53,8 v.H.; Statistisches Bundesamt (2000c): 51,6 v.H. bzw. (2003b) 54,5 v.H. Siehe auch: Wissenschaftlicher Beirat beim BMWi (1998, S.6).

4 Vgl. z.B.: Prognos (1998): 23,3 v.H. bis 23,7 v.H.; Wissenschaftlicher Beirat beim BMWi (1998, S. 38, Abb. 4): 25,5 v.H.; Sozialbeirat (1999, S. 145): 23,9 bis 25,9 v.H.; RürupKommission (2003, S. 99): 24,2 v.H.; Herzog-Kommission (2003; S. 59): 24,8 v.H. Vgl. z.B. Süddeutsche Zeitung (2003c). 
Mehr Eigenverantwortlichkeit und weniger Solidarität.

Die von der Rürup- und der Herzog-Kommission geforderte Stärkung der kapitalgedeckten Komponenten und die Zurückführung der umlagefinanzierten Anteile bedeutet eine Umverteilung der Lasten. In dem „Drei-Säulen-Modell““ der Weltbank ${ }^{6}$ wird die Gewichtung der einzelnen Tragsäulen modifiziert und damit das Gesamtgefuige restrukturiert. Während sich die Bedeutung der ersten Tragsäule, d.h. der staatlichen Regelsicherungssysteme, deutlich verringert, wird die zweite und dritte Säule, das ist die betriebliche und private Altersvorsorge, gestärkt.

Zur Umsetzung dieses Leitgedankens wird eine Absenkung des Rentenniveaus, eine Anhebung der Regelaltersgrenze und eine Streichung von Anreizen für Frühverrentungen gefordert. Insofern stellen diese Vorschläge zwar eine konsistente Fortführung der in den Rentenreformen von 1999 und 2001 angelegten Weichenstellungen dar, die Wirkungen sind jedoch wesentlich gravierender als bislang erörtert. Als besonders schwerwiegend sind hierbei die Einschnitte beim Rentenniveau zu bezeichnen. Als Folge der Kommissionspapiere soll sich beispielsweise das Brutto-Rentenniveau eines Eckrentners ${ }^{7}$ von derzeit (Stand 2001) rund 48,1 v.H. des Durchschnittseinkommens bis zum Jahr 2030 auf nur noch 42 v.H. bzw. 41,9 v.H. verringern. ${ }^{8}$ Wenngleich hierdurch die Funktion der Lebensstandardsicherung der bestehenden Alterssicherungssysteme als Ganzes nicht unmittelbar in Frage gestellt wird, eröffnen sich in Anbetracht der dann resultierenden Nähe des durchschnittlichen Rentenniveaus der gesetzlichen Rentenversicherung zum Sozialhilfesatz ${ }^{9}$ auch andere, d.h. systemverändernde Reformoptionen.

Als ein Lösungsansatz zur Abwendung der drohenden Finanzprobleme wird seit Jahren eine Umstellung auf ein Grundrentensystem mit pro Kopf einheit-

\footnotetext{
Vgl. World Bank (1995).
}

Eine Person mit Einkommen in Höhe des durchschnittlichen Einkommens aller sozialversicherungspflichtig Beschäftigten, die zum Zeitpunkt des Renteneintritts 45 Versicherungsjahre nachweisen kann.

8 Vgl. Rürup-Kommission (2003b), S. 98 bzw. Herzog-Kommission (2003), S. 71.

9 Die Absenkung des Rentenniveaus bedingt, dass die Durchschnittsrente im Jahr 2030 nur 4,2 v.H. und im Jahr 2050 nur 3,2 v.H. oberhalb des Sozialhilfeniveaus liegen wird. Vgl. Herzog-Kommission (2003), S. 71. 
lichen Leistungen diskutiert. ${ }^{10}$ Ein solches Konzept, welches die grundlegenden Ideen des englischen Politikers William Beveridge (1942) aufgreift, lässt sich insbesondere dadurch charakterisieren, dass das Regelsicherungssystem entsprechend des Grundsatzes der Subsidiarität nur existenzielle Risiken abdeckt. Die Wahrung des in der Erwerbsphase erreichten Lebensstandards erfolgt in einem Zusammenwirken aller Tragsäulen der Alterssicherung. Dabei nimmt die erste Sicherungssäule einen bedeutend geringeren Anteil als in den bestehenden Systemen ein. ${ }^{11}$ Die wesentlichen Vorteile einer Grundrente bestehen also neben der weitgehenden Transparenz des Rentensystems vornehmlich in den geringeren gesamtwirtschaftlichen Kosten. ${ }^{12}$

Wenngleich eine solche Neuordnung des Gesamtgefüges der Alterssicherung, d.h. die Gewichtsverlagerung zwischen den Säulen, durchaus Parallelen zum Votum der Rürup- oder der Herzog-Kommission aufweist, sind die Wirkungszusammenhänge gänzlich verschieden. ${ }^{13}$ Das konstitutive Merkmal eines solchen Rentensystems, die pro Kopf einheitliche Rente, bedingt eine Abkehr vom Äquivalenzprinzip ${ }^{14}$ und begründet damit einen Systemwechsel. Steht beispielsweise die Rente in der gesetzlichen Rentenversicherung in einem proportionalen Verhältnis zur Höhe der geleisteten Beiträge, existiert eine derartige Verknüpfung in einer Grundrente per definitionem nicht. Die hiermit verbundenen Verzerrungen der individuellen Arbeitsangebotsentscheidungen, die angesichts einer a priori feststehenden Rente eventuell verstärkt in den Vordergrund rückende Moral-Hazard-Problematik ${ }^{15}$ und insbesondere das Fehlen eines finanziell vertretbaren Weges einer Systemumstellung, der zugleich die

10 Vgl. z.B. Besendorfer et al. (1998), Biedenkopf, Miegel (1997), Burger (1996, 1997), Burgert et al. (1999), Buttler et al. (1987), Breyer (1998), Clausing (1985), Hauser (1993, 1996 und 2000), Miegel, Wahl (1999), Müller, Tautz (1996), Ruland (1999), Schmähl (1974, 1986, 1993a, b und 2000a), Wahl (1995 und 1999) und Wissenschaftlicher Beirat beim BMWi (1998).

11 Konkrete Ausgestaltungen eines Grundrentenmodells sind beispielsweise von Miegel, Wahl (1985) oder Die Grünen (1985) für Deutschland formuliert worden.

12 Angemerkt sei zudem, dass die Entkoppelung der Erwerbseinkommen von der Höhe der Renten auch als ein Beitrag zur geschlechtlichen Gleichberechtigung bewertet werden kann.

13 Es sei ausdrücklich darauf hingewiesen, dass die Rürup- und die Herzog-Kommission systemimmanente Reformen favorisieren. Ebenso: z.B. Wissenschaftlicher Beirat beim BMWi (1998).

14 Der theoretische Fall einer einheitlichen Kopfprämie für alle Bürger ohne kompensatorische Maßnahmen für einkommensschwache Bevölkerungsschichten wird aus naheliegenden sozialpolitischen Erwägungen ausgeschlossen.

15 Moral-Hazard beschreibt ein Trittbrettfahrertum. Wer arbeitet noch, wenn die Höhe der Rente fixiert und insbesondere unabhängig von der individuellen Beitragsleistung ist. 
verfassungsrechtlich geschützten Rentenansprüche und -anwartschaften der bestehenden Systeme aufrechterhält, ${ }^{16}$ sind die Hauptgründe, die zu einer Ablehnung eines solchen Rentensystems führen. ${ }^{17}$ Während die allokativen Verzerrungen einer Grundrente unumstößlich sind, ${ }^{18}$ ist die Stichhaltigkeit der beiden zuletzt genannten Argumente, die Moral-Hazard Problematik und die Möglichkeit zur Wahrung des Eigentumscharakters der bestehenden Rentenanwartschaften, maßgeblich von der Gesamtkonzeption des Sozialsystems und von der Ausgestaltung des Rentensystems bzw. eines konkreten Übergangsszenarios abhängig.

Die Zielsetzung der vorliegenden Arbeit besteht in einer eingehenden Analyse eines Grundrentenmodells auf der Grundlage von Mikrodaten des Sozioökonomischen Panels des Deutschen Instituts für Wirtschaftsforschung.

Schwerpunkte hierbei sind die Kosten einer Bürgerrente und die Wirkungen eines solchen Rentenmodells auf die Ruhestandseinkommen der Altersrentner. $\mathrm{Da}$ die Frage einer Implementierung eng mit der Existenz eines durchführbaren, d.h. finanziell tragfähigen Systemübergangs verknüpft ist, besteht eine weitere Hauptaufgabe in der Formulierung und der Analyse eines Übergangsszenarios. Die Zielsetzung besteht hierbei darin, die durch einen Systemwechsel induzierten Mehrbelastungen zu quantifizieren und die zeitliche Ausdehnung der Mehrbelastungen zu bestimmen. Als Grundlage der Analyse dient dabei eine in Anlehnung an Biedenkopf, Miegel (1997) konzipierte Bürgerrente. Dieses Modell hat die wissenschaftliche und politische Diskussion in der Bundesrepublik Deutschland über Jahre maßgeblich geprägt. ${ }^{19}$ Seine Analyse erlaubt begründete Schlüsse zu den erwähnten Fragen zu ziehen.

16 Nach geltender Rechtsprechung besteht für Rentenansprüche ein Eigentumsschutz nach Artikel 14 GG. Vgl. hierzu Art. 14 GG i.V.m. BverfG, Urteil vom 05.10.1983 - 1 BvR $820 / 79$. Ergänzend siehe auch Breyer (1998a) und die dort angegebene Literatur.

17 Vgl. z.B. Bundesministerium für Arbeit (1997), S. 6, Herzog-Kommission (2003), S. 40. Eine umfassende Kritik findet sich in z.B. in Schmähl (1988). Vgl. z.B. Breyer (1990) oder Fenge (1997).

Für weitere Fassungen des Modells siehe Miegel (1981), Miegel, Wahl (1985), Biedenkopf, Miegel (1997) oder Miegel, Wahl (1999). 
Hierzu ist die Arbeit in fünf Kapitel gegliedert:

Das erste Kapitel beschreibt die Grundgedanken der Biedenkopf-Miegelschen Bürgerrente. Aus der Beschreibung der Leistungen und Anspruchsvoraussetzungen eines solchen Rentensystems wird dieses Konzept als eine Tragsäule innerhalb eines teilkapitalgedeckten Rentenmodells charakterisiert. Außerdem werden die zu analysierenden Probleme deutlich. ${ }^{20}$

Im zweiten Kapitel erfolgt eine allgemeine Betrachtung der Altersicherung. Ausgehend von den Extrempositionen, einem reinen Umlage- und einem Kapitaldeckungsverfahren, werden die beiden wesentlichen Finanzierungsformen gegeneinander abgegrenzt und der zu beobachtende Renditeunterschied analysiert. In einer Zusammenführung der Erkenntnisse der theoretischen Literatur werden die Möglichkeiten eines pareto-effizienten Systemwechsels am Beispiel einer kleinen offenen Volkswirtschaft dargestellt.

Im dritten Kapitel werden die Kosten eines Grundrentensystems ermittelt. In einem ersten Teil werden die wesentlichen Vorgehensweisen und Ergebnisse früherer Kostenschätzungen diskutiert. Die Zielsetzung besteht dabei neben einer Aufarbeitung des Literaturstandes auch in einer Identifikation grundlegender Anforderungen an eine Modellierung zur Quantifizierung der Kosten eines Grundrentensystems. In einem zweiten Teilabschnitt wird ein neuer, eigenständiger Modellansatz formuliert und durchgerechnet. Als Folge der im ersten Teil festgestellten Kumulationsproblematiken stützt sich dieser im Wesentlichen auf Mikrodaten des Sozioökonomischen Panels (SOEP) des Deutschen Instituts für Wirtschaftsforschung Berlin (DIW). Mit dem SOEP ist es möglich, dass nach einer Auswertung der individuellen Rentenansprüche einzelner Befragungspersonen die Gesamtkosten eines Rentensystems aus einer repräsentativen Hochrechnung, d.h. aus einer Aggregation auf die Makroebene, folgen.

Im vierten Kapitel wird die Leistungsseite eines Rentensystems betrachtet. Die Zielsetzung besteht dabei in einem unmittelbaren Vergleich der Ruhestandseinkommen der Altersrentner in den beiden Rentensystemen. Hierzu werden die Daten des SOEP zur Erwerbsbiographie in Tätigkeitsprofile überführt und die resultierenden Rentenansprüche in Rentenkonten zusammengefasst. Zur Systematisierung der Analyse werden hierzu in einem ersten Teilabschnitt typische Erwerbsbiographien identifiziert. In einem zweiten Teilabschnitt erfolgt dann die vergleichende Analyse der Ruhestandseinkommen.

Vorarbeiten hierzu finden sich in Adamski (1999). 
Im fünften Kapitel erfolgt aus den in Kapitel 3 erarbeiteten Zahlen des SOEP die Projektion der Kosten eines Rentensystems. Auf der Grundlage unterschiedlicher Szenarien zur ökonomischen und demographischen Entwicklung in Deutschland werden die Kosten einer Bürgerrente und die der bestehenden Systeme bis zum Jahr 2040 fortgeschrieben. Die Analyse ist dabei im Wesentlichen in zwei Teilabschnitte gegliedert: Nach einer Vorausberechnung der Kosten eines eingeführten Rentensystems erfolgt die Modellierung eines Systemübergangs. Hierdurch ergibt sich die Möglichkeit, die zukünftige Kostenentwicklung einer Bürgerrente detailliert zu analysieren. Außerdem kann die Systemüberführung, die mit wesentlichen größeren Freiheitsgraden verbunden ist, eingehend untersucht werden.

Die quantitative Auswertung der SOEP-Daten erfolgt durch ein komplexes, aufeinander aufbauendes Programmgeflecht, welches sich neben standardisierten Algorithmen (z.B. Clusteranalyse, Optimal Matching) vorwiegend auf eigenständig erstellte Algorithmen stützt. Angewendet werden hierzu im Wesentlichen die Sprachen beziehungsweise Softwarepakete Visual Basic for Application (VBA), SPSS-Syntax und Transitional Data Analysis (TDA). Ein Überblick über grundlegende Programmstrukturen ist im Anhang zu finden. 


\section{Die Bürgerrente nach Biedenkopf}

\subsection{Die Grundgedanken}

Als Alternative zum gegenwärtigen Rentensystem, das maßgeblich durch die gesetzliche Rentenversicherung (GRV) geprägt wird, schlagen Biedenkopf, Miegel (1997) ${ }^{21}$ den Wechsel zu einem allgemeinen, für alle Bürger obligatorischen Versorgungssystem ${ }^{22}$ mit ergänzender, privatwirtschaftlich organisierter Kapitaldeckung vor, eine sogenannte Bürgerrente.

Hierbei handelt es sich um ein in Anlehnung an den Beveridge-Report ${ }^{23}$ konzipiertes Rentenkonzept mit individualisierten Leistungen, in dem jeder Berechtigte, ohne Prüfung einer potenziellen Bedürftigkeit, Anspruch auf den Bezug einer pro Kopf einheitlichen Leistung im Versicherungsfall (Alter, Invalidität oder Hinterbliebener) besitzt. Da die erforderlichen Mittel durch das Steueraufkommen einer jeweiligen Periode (direkte und indirekte Steuern) gedeckt werden, erfolgt die Finanzierung des obligatorischen Teils wie in den bestehenden Systemen in einem Umlageverfahren. Aber eine Beitragsäquivalenz, d.h. ein proportionales Verhältnis der eingezahlten Beiträge zur Höhe der zu erwartenden Rentenleistungen, ist vom Grundsatz her nicht gegeben.

Die Höhe einer Bürgerrente orientiert sich an einem sozialen Existenzminimum, welches nach üblicher Auffassung durch den Bedarf nach dem Bundessozialhilfegesetz als definiert anzusehen ist. Die Zielsetzung der Bürgerrente besteht somit nicht in einer Lebensstandardsicherung, sondern in einer Sicherung eines sozio-kulturellen Existenzminimums. Die Fortführung des Lebensstandards im Ruhestand ist folglich nur in Kombination mit eigenständigen Vorsorgebestrebungen, d.h. durch privates Sparen in der Erwerbsphase, möglich. Das System der Bürgerrente zeichnet sich also insbesondere dadurch aus, dass den „Versicherten“ ein größeres $\mathrm{Ma} ß$ an Eigenständigkeit geboten beziehungsweise von diesen gefordert wird. Kurzum, das Konzept der Bürgerrente orientiert sich an dem Leitgedanken der Subsidiarität, wonach die Solidargemeinschaft nur das leisten soll, was der Einzelne nicht zu leisten vermag.

Für ältere Vorschläge siehe auch: Miegel (1981), Miegel, Wahl (1985) und (1999). Kritisch: Schmähl (1988).

In Anlehnung an z.B. Schmähl (1996, 23f.) sei unter einem „Versorgungssystem“ eine Alterssicherungskonzeption verstanden, in der eine Beitragsäquivalenz nicht gegeben ist. Im Unterschied hierzu lässt sich die gesetzliche Rentenversicherung als ein „Vorsorgesystem" beschreiben, da hier die Rentenhöhe prinzipiell durch die Vorleistungen in der Erwerbsphase bestimmt wird.

Vgl. Beveridge (1942). 
In dem Modellschema der Weltbank, dem Drei-Säulen-Modell ${ }^{24}$, bedeutet die Einführung einer Bürgerrente eine Verlagerung innerhalb des Gesamtgefüges der Alterssicherung auf die Säulen zwei und drei, d.h. weg von der ersten Säule, den staatlichen Regelsicherungsinstitutionen, hin zur betrieblichen und privaten Vorsorge. Die Trennlinie zwischen Eigenverantwortung und Solidarität wird im Vergleich zum Status quo neu definiert.

Die Bürgerrente lässt sich somit als ein steuerfinanziertes und im Wesentlichen von der individuellen Beitragsleistung unabhängiges Regelsicherungssystem mit ergänzender, privatwirtschaftlich organisierter Kapitaldeckung charakterisieren, welches die erste Säule der bestehenden Alterssicherungssysteme substituiert. Die wesentlichen Eckpunkte des Biedenkopf-Miegelschen Vorschlags sind in den nachfolgenden Abschnitten zusammengefasst.

\subsection{Leistungen und Anspruchsvoraussetzungen}

Die Bürgerrente garantiert jedem Berechtigten den Bezug eines Jahreseinkommens in vom Hundert des durchschnittlichen Volkseinkommens pro Kopf der Wohnbevölkerung. ${ }^{25}$ Im Falle des Biedenkopf-Miegelschen Vorschlags betragen die Leistungen 55 v.H. Die Koppelung der Bürgerrente mit dem Volkseinkommen bewirkt dabei, dass die Renten proportional zur allgemeinen Einkommensentwicklung, im Unterschied zur GRV auch der Kapitaleinkommen, wachsen. Nach Abzug der Beiträge zur Kranken- und Pflegeversicherung der Rentner (KVdR/PVdR), die in einer Abkehr von der paritätischen Finanzierung ausschließlich von den Rentnern zu tragen sind, errechnet sich beispielsweise für das Jahr 1999 ein Monatseinkommen in Höhe von rund $€ 695^{26}$ pro Person. Da auf Einkommen in Höhe der Bürgerrente keine Steuern zu entrichten sind, beschreibt diese Größe das Nettoeinkommen. Infolge der Ausgestaltung der Bürgerrente als einen individualisierten Leistungsanspruch kann ein empfangsberechtigtes Rentnerpaar über ein Gesamteinkommen in Höhe von $€ 1390$ verfügen.

24 Vgl. World Bank (1995).

25 Vgl. Biedenkopf, Miegel (1997), S. 12. Andere Vorschläge in einer Bandbreite von rund 40 v.H. bis 52,5 v.H. finden sich bei Miegel (1981, S. 103), Miegel, Wahl (1985, S. 67 und 1999, S. 127).

Annahmen: Volkseinkommen in Höhe von $€ 17844$. Beiträge zur KVdR / PVdR in Höhe von 15 v.H. 
Empfangsberechtigt ist jeder Bürger ${ }^{27}$, der im Leistungsfall, d.h. im Alter, als Hinterbliebener oder als Erwerbsgeminderter die in der Tabelle 1 aufgeführten Voraussetzungen erfüllt:

Voraussetzungen zum Bezug einer Bürgerrente

\begin{tabular}{cll}
\hline Anspruchsgrundlage & \multicolumn{1}{c}{ Voraussetzung für den Bezug } \\
\hline Altersrente & $\bullet$ & Vollendung des 65. Lebensjahres \\
& $\bullet$ & 25 Jahre Steuerpflichtigkeit nach Volljährigkeit \\
\hline $\begin{array}{c}\text { Erwerbsminderungs- } \\
\text { rente }\end{array}$ & $\bullet$ & 5 Jahre Steuerpflichtigkeit \\
\hline Witwen- oder & 5 Jahre Erwerbstätigkeit \\
Witwerrente & $\bullet$ & Vollendung des 55. Lebensjahres \\
& $\bullet$ & Mindestens fünfjährige Nichterwerbstätigkeit \\
& vor Eintritt des Versorgungsfalles \\
\hline Waisen- oder & $\bullet$ & 5 Jahre Steuerpflichtigkeit mindestens eines \\
Halbwaisenrente & Elternteils nach dessen Volljährigkeit und vor \\
& Eintritt des Versorgungsfalles \\
\hline Renten an Erzieher & $\bullet$ & Der zu Erziehende hat selbst Anspruch auf den \\
von Waisen oder & Bezug der Bürgerrente \\
Halbwaisen & Der zu Erziehende ist nicht älter als 12 Jahre \\
\hline
\end{tabular}

Tabelle 1: Voraussetzungen zum Bezug Bürgerrente

Eine Bürgerrente wegen Alters kann ab dem vollendeten 65. Lebensjahr bezogen werden, sofern eine mindestens 25-jährige Steuerpflichtigkeit in der Bundesrepublik Deutschland nachgewiesen werden kann. Die Leistungsgewährung orientiert sich folglich neben einer Festlegung eines frühesten Renteneintrittsalters an einem lebenslaufbezogenen Territorialprinzip. ${ }^{29}$ Nur derjenige, der innerhalb der Erwerbsphase langjährig der uneingeschränkten Steuerpflichtigkeit in der Bundesrepublik Deutschland unterliegt, d.h. seinen gewöhnlichen

27 D.h. es handelt sich um ein Rentensystem, das alle Personen, auch bislang nicht gesetzlich versicherte Selbstăndige oder Beamte, erfasst. Die Darstellung der Grundsätze einer Bürgerrente konzentriert sich jedoch auf die Unterschiede zur GRV. Biedenkopf, Miegel (1997) sprechen von einer Erwerbsunfähigkeit. Infolge der inzwischen geänderten Rechtslage wird von einer Erwerbsminderung nach § 43 SGB VI ausgegangen. Vgl. hierzu Hauser (1993), S.77f. 
Wohnsitz im Inland unterhält, verfügt im Alter über Ansprüche auf Leistungen des obligatorischen Alterssicherungssystems. Die Bindung des Anspruches an einen „Bürgerbegriff“ wirft allerdings definitorische Fragen auf. Wird dieser mit einem Staatsbürger nach Artikel 116 GG gleichgesetzt, bezöge sich der Leistungsanspruch nur auf Personen mit deutscher Staatsangehörigkeit. Dies erscheint jedoch vor dem Hintergrund der Formulierung eines obligatorischen Rentensystems und angesichts der großen Anzahl ausländischer Mitbürger mit dauerhaftem Wohnsitz in der Bundesrepublik Deutschland wenig sinnvoll. Geeigneter ist ein Verständnis eines „Bürgers“ im Sinne eines Inländers. Für die Gewährung einer Bürgerrente bedeutet dies, dass prinzipiell auch ein altersbezogenes Territorialprinzip zu erfüllen ist. ${ }^{30}$

Proratisierungen, wie sie z.B. in anderen Staaten mit Grundrentenmodellen zu finden sind, ${ }^{31}$ sind von Biedenkopf, Miegel (1997) nicht vorgesehen. Hierdurch sei sichergestellt, dass nur Personen, die zur Finanzierung des Systems beigetragen haben, Ansprüche erwirken können. Zusätzliche Anreize für eine Migration entstünden daher nicht.

Im Falle einer Erwerbsminderung besteht neben dem Nachweis einer mindestens fünfjährigen Steuerpflichtigkeit in der Bundesrepublik nur die Erfordernis, eine ebenso lange Erwerbstätigkeit zu belegen, um empfangsberechtigt zu sein. Im Vergleich zu den Regelungen in der gesetzlichen Rentenversicherung, nach denen ein Anspruch wegen einer Erwerbsminderung an eine mindestens dreijährige Erwerbstätigkeit in den letzten fünf Jahren unmittelbar vor dem Leistungsfall gekoppelt ist, ergeben sich kaum wesentliche Änderungen.

Bei Tod eines Ehepartners ist der Leistungsanspruch hingegen deutlich eingeschränkt. Lediglich Personen in einem Lebensalter von 55 bis unter 65 Jahren sind anspruchsberechtigt, sofern eine mindestens 25-jährige Steuerpflichtigkeit und eine fünfjährige Nichterwerbstätigkeit unmittelbar vor Eintritt des Leistungsfalles nachgewiesen werden kann. Die Bürgerrente orientiert sich folglich an einem Familienbild, in dem jeder „Versicherte" individualisierte Ansprüche besitzt und daher eigenständig für sich sorgen muss. Eine Absicherung über den Ehepartner ist nicht oder nur bedingt vorgesehen. ${ }^{32}$ Dies kann zumindest in Teilen als ein Beitrag zur geschlechtlichen Gleichberechtigung verstanden werden.

30

Vgl. Hauser (1993), S. 77f.

Vgl. z.B. Kanada "Old-Age-Security (OAS)". Ähnliches gilt auch für die Grundrentensysteme in Großbritannien, Finnland, Irland und Dänemark. Einen detaillierten Überblick bietet Bundesministerium für Gesundheit und Soziale Sicherung (2003).

Siehe auch: Schmähl (1993b), S. 279. 
Der Leistungsanspruch von Waisen und Halbwaisen ist im Wesentlichen in Anlehnung an die bestehenden Regelungen der gesetzlichen Rentenversicherung ausgestaltet. ${ }^{33}$ Die Zahlung der Bürgerrente wird bis zum Ende der Ausbildung oder alternativ bis zum vollendeten 27. Lebensjahr in Höhe des halben Regelsatzes gewährt. Als zusätzliche Anspruchsvoraussetzung ist auch hier der Nachweis einer mindestens fünfjährigen Steuerpflichtigkeit eines Elternteils erforderlich.

Ähnliches gilt für Erzieher von Waisen und Halbwaisen. Diese zählen zu den Empfangsberechtigten, sofern der zu Erziehende selbst einen Anspruch auf die Bürgerrente besitzt und das 12. Lebensjahr noch nicht vollendet hat. Die Leistungshöhe ist ebenfalls auf den halben Regelsatz der Bürgerrente begrenzt.

Die Leistungsgewährung einer Bürgerrente zeichnet sich also insbesondere dadurch aus, dass neben dem Nachweis eines „Versicherungsfalles“, hier z.B. die Vollendung des allgemeinen Verrentungsalters, die Feststellung einer Erwerbsminderung oder der Tod eines Angehörigen, generell die Erfüllung des lebenslaufbezogenen Territorialprinzips zu belegen ist.

\subsection{Finanzierung}

Die Bürgerrente ist ein ausschließlich steuerfinanziertes Rentenkonzept, welches die bislang bestehende Mischfinanzierung aus Steuern und / oder lohnabhängigen Beiträgen ersetzt. Die erforderlichen Mittel werden durch eine umfangreiche Umschichtung in der Beitrags- und Steuerbelastung der Haushalte bereitgestellt. Während einerseits eine signifikante Anhebung der direkten und indirekten Steuersätze erforderlich ist, entfallen im Gegenzug die Arbeitnehmeranteile zur Rentenversicherung vollständig. Darüber hinaus werden 70 v.H. der Arbeitgeberanteile an die Erwerbstätigen ausgezahlt, um diese zu entlasten. Zudem soll hierdurch den Individuen die Möglichkeit zur eigenständigen Vorsorge eingeräumt werden. Die übrigen 30 v.H. der Arbeitgeberanteile verbleiben bei den Unternehmen.

Zur Deckung des von Biedenkopf, Miegel berechneten Gesamtvolumens einer eingeführten Bürgerrente in Höhe von rund $€ 140 \mathrm{Mrd}$. (oder rund 8 v.H. des Bruttoinlandsproduktes) ${ }^{34}$ im Jahr 1995 sei zwar eine Steigerung der direkten

Sofern in Analogie zu den bestehenden Systemen ein Großteil der 60 bis 65-Jährigen zu den Empfangsberechtigten zählen, erhöhe sich das Finanzvolumen auf rund $€ 159$ Mrd. 
Steuersätze um 15 v.H. und der indirekten um 22 v.H. notwendig. ${ }^{35}$ Infolge der Entlastung verbleibe aber insgesamt eine erhöhte Sparfähigkeit, die für einen unverheirateten (verheirateten) Durchschnittsverdieners $€ 2045$ ( $€$ 2557) pro Jahr betrage. Sofern die zusätzliche Sparfähigkeiten zur Bildung eines Kapitalstockes verwendet werden, ergäbe sich bis zum Renteneintritt im Jahr 2035 (d.h. nach einer Dauer von 40 Jahren) ein Geldbetrag in Höhe von rund $€ 204517$ bzw. $€ 255646 .{ }^{36}$ Hieraus könnten die beispielhaft genannten Personen ergänzend zur Bürgerrente ein Einkommen in Höhe von rund $€ 1023$ bzw. $€ 1278$ erzielen. ${ }^{37}$

\subsection{Der Systemübergang}

Die Einführung einer Bürgerrente bedeutet im Modellschema der Weltbank eine Ablösung der ersten Säule der bestehenden Alterssicherungssysteme. Da bereits erworbenen Rentenanwartschaften ein Eigentumscharakter nach Artikel 14 GG zugesprochen wird, ${ }^{38}$ sind diese auch bei einer Systemveränderung aufrechtzuerhalten.

Hierzu werden alle fälligen und nichtfälligen Ansprüche (z.B. die zahlungswirksame Altersrente eines 70-Jährigen oder die Rentenanwartschaft eines Erwerbstätigen) eines Individuums auf Leistungen der bestehenden Rentensysteme zum Zeitpunkt der Systemumstellung (im Sinne eines Stichtagverfahrens) ermittelt und in einem Geldbetrag zusammengefasst. Dieser kann dann als Anteil des Regelsatzes einer Bürgerrente ausgedrückt werden. Sofern der Gesamtanspruch den Regelsatz einer Bürgerrente nicht übersteigt, wird dieser mit jährlich gleichbleibenden Raten in Höhe von 4 v.H. des Regelsatzes aufgestockt. Andernfalls bleiben die Ansprüche erhalten und werden mit der Wachstumsrate des Volkseinkommens dynamisiert. Da die Fähigkeit zur eigenständigen Vorsorge maßgeblich von der Zeitspanne bis zum Ruhestandseintritt bestimmt wird, werden bei Personen zwischen dem vollendeten 40 . und dem 65. Lebensjahr nichtfällige Ansprüche nach Erreichen des Regelsatzes mit dem halben Aufstockungsbetrag bis zum Ruhestandseintritt fortgeschrieben.

35 Hieraus ergäbe sich eine Mehrwertsteuer in Höhe von gut 18 v.H. und ein Spitzensteuersatz der Einkommenssteuer in Höhe von 52 v.H.

Bei Annahme eines konstanten Zinses in Höhe von 3 v.H. und einer Spardauer von 40 Jahren. Alle Angaben im Geldwert von 1995.

Alle Angaben im Geldwert von 1995.

Vgl. Art. 14 GG i.V.m. BverfG, Urteil vom 05.10.1983 - 1 BvR 820/79. 
Zur Illustration seien die folgenden Beispiele betrachtet ${ }^{39}$ :

I Zum Zeitpunkt der Umstellung verfüge eine Person im Ruhestand über eine Gesamtrente in Höhe von 80 v.H. des Regelsatzes einer Bürgerrente. Innerhalb der nächsten fünf Jahre wird dieser Betrag um jährlich 4 v.H. des Zahlbetrages auf den Regelsatz aufgestockt.

II Die nichtfälligen Ansprüche einer 45-jährigen Person mögen sich auf 60 v.H. des Regelsatzes summieren. Infolge der Aufstockungen wird der Regelsatz mit 55 Lebensjahren erreicht. Bis zum Renteneintritt mit 65 Jahren steigen die Ansprüche dann um jährlich 2 v.H., so dass sich insgesamt ein Niveau in Höhe von 120 v.H. des Regelsatzes ergibt.

III Eine erwerbstätige Person im Alter von 50 Jahren verfüge innerhalb der gesetzlichen Rentenversicherung über eine Hinterbliebenenversorgung in Höhe von 16 v.H. des Regelsatzes. Aufgrund eigener Berufstätigkeit hat die Person bereits Rentenanwartschaften in Höhe von 44 v.H. erwirkt, die erst mit Eintritt in den Ruhestand zahlungswirksam werden. $\mathrm{Da}$ die fälligen und nichtfälligen Ansprüche mit 4 v.H. pro Jahr aufgestockt werden, wird der Regelsatz einer Bürgerrente mit 60 Lebensjahren erreicht. Bis zum Renteneintritt mit 65 Jahren steigen die nichtfälligen Ansprüche mit 2 v.H. weiter an. Es ergibt sich somit ein Gesamtanspruch in Höhe von 110 v.H. der Bürgerrente.

Die Beispiele belegen, dass die Bürgerrente insbesondere die Empfänger kleiner Renten begünstigt. Diese werden innerhalb kurzer Zeit auf den Regelsatz aufgestockt. Da zudem die Altansprüche bestehen bleiben, ergibt sich in der Phase des Systemübergangs ein erhöhter Finanzbedarf. Biedenkopf, Miegel (1997) schätzen den zusätzlichen Bedarf auf rund $€ 4,1 \mathrm{Mrd}$. Die Finanzierung der Umstellungskosten erfolgt durch weitere Steuererhöhungen.

\subsection{Zusammenfassung}

Die Bürgerrente ist ein ausschließlich steuerfinanziertes Rentensystem, das auf dem Versorgungsgedanken aufbaut. Prinzipiell jeder Bürger hat im Leistungsfall, d.h. im Alter, bei einer Erwerbsminderung oder im Todesfall eines Angehörigen Anspruch auf den Bezug einer pro Kopf einheitlichen Leistung, ohne dass eine Bedürftigkeit nachgewiesen werden muss. Bei der Bürgerrente handelt es sich also um eine obligatorische Leistung des Staates, nicht um Al- 
mosen. Hierdurch ist das neue System in der Lage, Altersarmut wirkungsvoll zu bekämpfen.

Darüber hinaus stellt das Gesamtkonzept erhebliche Einsparungen im Vergleich zu einer Fortführung der bestehenden Systeme in Aussicht. Biedenkopf, Miegel (1997) sprechen von rund einem Drittel der derzeitigen Aufwendungen.

Diese Einsparpotentiale entstehen vornehmlich dadurch, dass die Aufgabe des obligatorischen Rentensystems auf die Sicherung eines sozio-kulturellen Existenzminimums beschränkt wird. Die Wahrung des erreichten Lebensstandards, einer zweiten Funktion der bestehenden Systeme, wird nur in einer Kombination mit sekundären Sicherungsinstrumenten, z.B. betrieblicher Alterssicherung oder privater Vorsorge ermöglicht. Der Leitgedanke der Subsidiarität wird somit eindrucksvoll umgesetzt.

Kurzum, das System der Bürgerrente lässt sich auch als ein umfassender Beitrag zur Deregulierung und Privatisierung der sozialen Sicherung auffassen. ${ }^{40}$ Der Staat beschränkt sich auf die Absicherung eines Mindestmaßes und überlässt den Bürgern die Möglichkeit, mehr Eigenverantwortlichkeit zu übernehmen. Das von Biedenkopf, Miegel (1997) vorgelegte Papier bietet einen interessanten Diskussionsansatz, der einer detaillierten Untersuchung bedarf. Dies gilt umso mehr, da das vorgestellte Konzept vielfältige Möglichkeiten zur praktischen Ausgestaltung beinhaltet.

In der vorliegenden Arbeit werden die Biedenkopf-Miegelschen Anregungen daher nicht als starres Korsett, sondern als Grundsatzpapier verstanden, in dem noch eine große Anzahl an Detailfragen zu klären oder zu regeln sind, ohne den Grundgedanken zu beeinträchtigen. Im Einzelnen wird in der nachfolgenden Analyse ein Grundrentensystem untersucht, welches auf den Leitgedanken Biedenkopf, Miegels (1997) aufbauend den Anschluss an die grundsätzlichen Überlegungen Beveridges (1942) findet, d.h. es wird ein beitragsfinanziertes System einer Bürgerrente untersucht. Hierdurch gelingt es, die Wirkungsweisen einer Bürgerrente auf die finanzielle Situation der Rentner aufzuzeigen und zugleich eine wesentlich höhere Vergleichbarkeit zu den bestehenden Gegebenheiten zu erreichen, als dies bei einer ausschließlich steuerfinanzierten Grundrente möglich wäre. 


\section{Grundtypen der Alterssicherung - Allgemeine Aussagen aus der Theorie}

Das Gesamtkonzept der Biedenkopf-Miegelschen Bürgerrente lässt sich als ein umlagefinanziertes Rentensystem mit pro Kopf einheitlichen Leistungen und ergänzender, privater Kapitaldeckung charakterisieren. Mit anderen Worten die Umstellung der bestehenden Rentensysteme, die vornehmlich durch die gesetzliche Rentenversicherung geprägt sind, auf eine Bürgerrente führt zu einer Veränderung des Finanzierungsverfahrens. An die Stelle eines reinen Umlageverfahrens ${ }^{41}$ tritt ein Mix aus Umlage- und Kapitaldeckungsverfahren. Ob ein derartiger Wechsel durchführbar ist, ohne Nachteile für eine Generation zu bewirken, wird in den folgenden Abschnitten untersucht.

\subsection{Umlage- versus Kapitaldeckungsverfahren}

Das Umlage- und das Kapitaldeckungsverfahren sind die grundlegenden Finanzierungsformen eines Rentensystems. Wenngleich die Bürgerrente eine intermediäre Stellung einnimmt, erscheint es aus methodischen Erwägungen sinnvoll, die Extrempositionen gegeneinander abzugrenzen. ${ }^{42}$

Ein Umlageverfahren (UV) zeichnet sich dadurch aus, dass die Mittel für die Zahlungen an die Rentnergeneration durch Beiträge der Erwerbstätigen der selben Periode aufgebracht werden. Grundsätzlich gelte in jeder Periode eine Identität von Einnahmen und Ausgaben. Ein Kapitalstock wird nicht akkumuliert. Die intergenerationale Umverteilung bedingt unvermeidliche Einführungsgewinne, da die Anfangsgeneration Leistungen erhält, ohne Beiträge entrichtet zu haben. ${ }^{43}$ Umgekehrt gäbe es im Falle einer Beendigung des UV eine Generation, die trotz einer Beitragsleistung keine Rentenzahlung zu erwarten hätte. Ein charakterisierendes Merkmal eines Umlageverfahrens ist folglich die implizite Staatsschuld, die den nachfolgenden Generationen aufgebürdet wird.

\footnotetext{
41 Im Hinblick auf die Bestimmungen des Altersvorsorgevermögensgesetzes gilt diese Aussage nur bedingt. Vgl. hierzu Deutsche Bundesregierung (200lc).

M.a.W. auch für das System der gesetzlichen Rentenversicherung erfolgt eine weitreichende Abstraktion.

43 Beispiele hierfür sind z.B. die Rentnergeneration zu Beginn der GRV ab dem Jahr 1957 oder die Rentner in den neuen Bundesländern, die im Zuge der deutschen Vereinigung in das System der GRV integriert worden sind.
} 
Die Rendite eines Umlageverfahrens, d.h. die Verzinsung der gezahlten Beiträge, wird von der Entwicklung der Beitragsleistungen und dem Bevölkerungswachstum bestimmt. Für den Fall einer gleichgewichtigen Ökonomie mit lohnproportionalen Beiträgen zum Rentensystem entspricht die Rendite mit Aaron (1966) der Summe der Wachstumsraten der Bevölkerung und der Löhne, d.h. der Lohnsummenwachstumsrate. ${ }^{44}$

In einem Kapitaldeckungsverfahren (KDV) hingegen wird ein Kapitalstock gebildet. Die Beiträge der Erwerbstätigen werden zum Kapitalmarktzins angelegt und in der Folgeperiode zur Finanzierung der Rentenzahlungen verwendet. Es kommt also zu einer intertemporalen Umverteilung innerhalb der selben Generation. Die Rendite eines KDV entspricht somit dem Kapitalmarktzins.

Wird nun mit z.B. Siebert (1998, S. 8) die Rendite eines Rentensystems als ein Bewertungskriterium betrachtet, gilt die Spremann-Regel (1984, S. 242f.): Ein Umlageverfahren ist gegenüber einem Kapitaldeckungsverfahren pareto-dominant, wenn die Lohnsummenwachstumsrate den Zinssatz übersteigt.

Die für Deutschland relevante Situation ergibt sich aus Abbildung 1. Im Einzelnen sind die realen Renditen der gesetzlichen Rentenversicherung (dünne Linien, linke Ordinate) und die Rendite eines alternativen Kapitaldeckungsverfahrens (dünne Linie mit Rauten, linke Ordinate), hier exemplarisch die reale jährliche Verzinsung zehnjähriger Bundesanleihen, für Standardrentner verschiedener Geburtskohorten eingezeichnet. Diese Rentner zeichnen sich dadurch aus, dass sie mit Vollendung des 20. Lebensjahres eine Erwerbstätigkeit beginnen und diese im Prinzip ${ }^{45}$ bis zum Renteneintritt im Alter von 65 Jahren ununterbrochen ausüben. ${ }^{46}$ Die interne Rendite der GRV ist in einer makroökonomischen Perspektive in Form der realen Lohnsummenwachstumsrate (dünne, unterbrochene Linie, linke Ordinate) und aus einer mikroökonomischen Sichtweise als reale Verzinsung der Beitragsleistungen (dünne, durchgezogene Linie), die der Standardrentner auf dem Kapitalmarkt erzielen

44 Dies gilt freilich nur dann, wenn die erste und die letzte Generation außer Acht bleiben. Samuelson (1958, S. 472) spricht für ein UV mit konstanten Löhnen von einem „biologischen Zinssatz".

45 Hierbei handelt es sich um einen Erwartungswert, da auch die Möglichkeit einer Erwerbsminderung berücksichtigt wird. Für eine detaillierte Beschreibung vgl. Wissenschaftlicher Beirat beim BMWi (1998, S. 20ff.).

46 Die Biographie des Standardrentners verdeutlicht, dass es sich hierbei offenkundig nicht um die Vorstellung einer qualifiziert ausgebildeten oder studierten Person handeln kann. Vielmehr wird ein Lebenslauf einer Person mit niedrigem Bildungsabschluss angenommen, der so nur für eine Minderheit in der Bevölkerung Relevanz besitzt. 
müsste, um ein Alterseinkommen in Höhe der zu erwartenden GRV-Rente zu beziehen, angegeben. Wie Abbildung 1 belegt, stimmen diese annähernd überein, liegen jedoch signifikant unterhalb der Kapitalmarktrendite.

\section{Renditen eines Rentensystems im Vergleich KDV versus GRV}

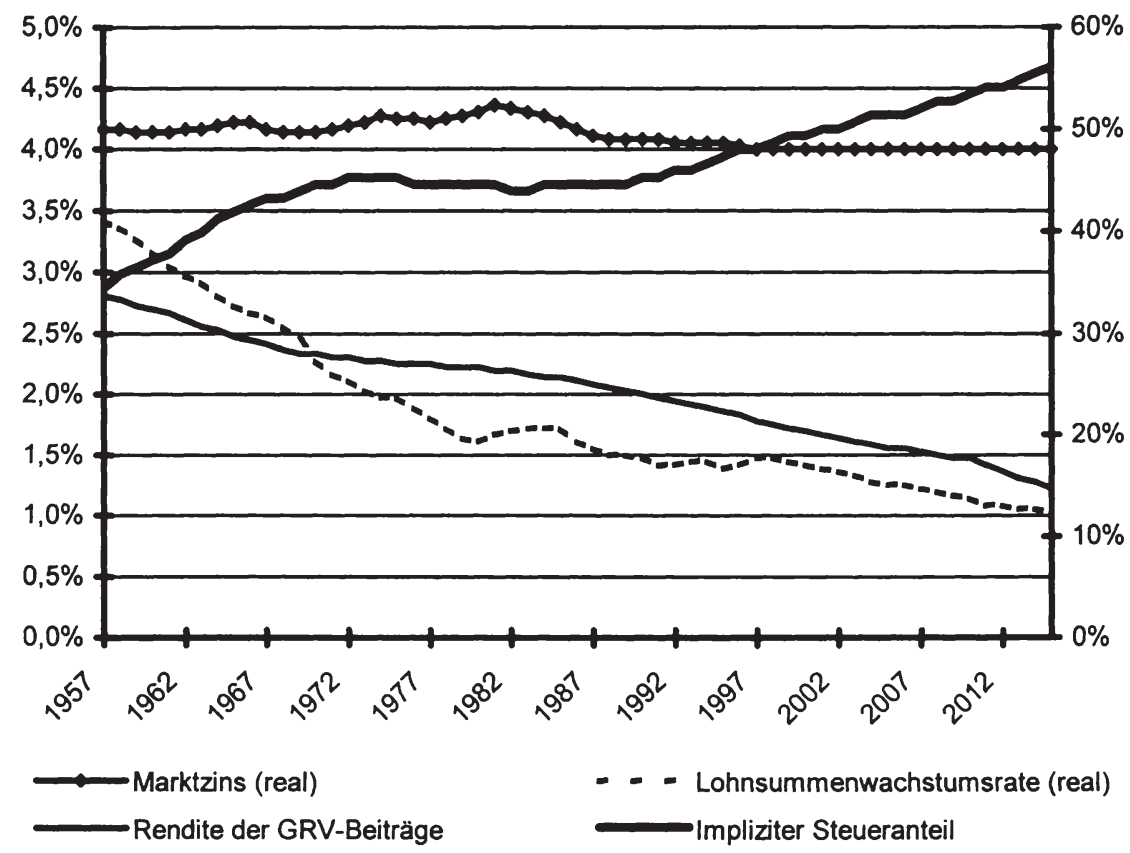

Abbildung 1: Renditen eines Rentensystems im Vergleich - KDV versus GRV ${ }^{47}$

Die GRV bietet einem Standardrentner, der im Gründungsjahr 1957 eine Erwerbstätigkeit aufnimmt, eine reale Verzinsung der Beitragsleistungen in Höhe von rund 2,8 v.H. Alle späteren Kohorten erzielen aufgrund der demographischen Veränderungen geringere Renditen. Für Standardrentner der Geburtskohorte 1980, die im Jahr 2000 eine Erwerbstätigkeit aufnehmen, beträgt die reale interne Rendite der gesetzlichen Rentenversicherung beispielsweise nur

47 Quelle: Wissenschaftlicher Beirat beim BMWi (1998), S. 21. Ähnliche Ergebnisse präsentieren auch Buslei, Kraus (1996), S. 63. 
rund 1,6 v.H. Im Vergleich hierzu ist die interne Systemrendite eines Kapitaldeckungsverfahren mit rund 4 v.H. bis 4,3 v.H. für alle Geburtskohorten deutlich höher. ${ }^{48}$

Kurzum, die interne Rendite eines Kapitaldeckungsverfahrens übersteigt die eines Umlageverfahrens, d.h. ein KDV pareto-dominiert ein UV. ${ }^{49}$ Fraglich ist jedoch, ob es ein intergenerationales Umverteilungsschema gibt, durch das die Übergangsgeneration entschädigt werden kann, ohne mindestens eine nachfolgende Generationen im Vergleich zu einem Verbleib im ursprünglichen Umlageverfahren $\mathrm{zu}$ benachteiligen, und durch das mindestens einer Generation Vorteile gewährt werden können. Fraglich ist also die Existenz eines pareto-effizienten Systemübergangs.

Notwendige Bedingung hierfür sind Ineffizienzen in der Ausgangssituation, die durch eine Abkehr vom UV abgebaut oder zumindest verringert werden können. Der folgende Abschnitt konzentriert sich dabei auf Denkansätze, bei denen die Ineffizienzen durch das Umlageverfahren selbst begründet sind, zum Beispiel infolge allokativer Verzerrungen des Arbeitsangebotes. ${ }^{50}$ Zudem gelte stets, dass die betrachtete Ökonomie relativ zum realen Zinssatz schrumpft. ${ }^{51}$

\subsection{Möglichkeiten eines pareto-effizienten Systemübergangs}

In der Ausgangssituation sei ein Umlageverfahren etabliert, in dem die Rentenbeiträge in Form einer lohnunabhängigen Pauschalsteuer (z.B. einer Kopfsteuer) von den Erwerbstätigen erhoben und als Pauschalrente an die Rentner ausgezahlt werden. Das Arbeitsangebot sei exogen. Um die Generation der Rentner bei einem Wechsel zu einem Kapitaldeckungsverfahren für die entgangenen Rentenzahlungen des ursprünglichen UV zu entschädigen, wird eine Staatsschuld aufgenommen. Für alle folgenden Generationen gilt das KDV, welches annahmegemäß eine größere Rendite und damit einen Nutzenvorteil

Angemerkt sei, dass die Bindung des realen Marktzinses an zehnjährige Bundesanleihen nicht zwangsläufig ist. Als Vergleichsmaßstab sind durchaus die Renditen anderer Anlageformen denkbar. Einen Überblick über reale Renditen auf dem Kapitalmarkt bietet z.B. Börsch-Supan (1998c), S. 412. Vgl. hierzu Balasko, Shell (1980) S. 286 und Spremann (1984), S. 243f.

so Ausführlichere Darstellungen der wissenschaftlichen Diskussion finden sich z.B. bei Buslei, Kraus (1996) oder Bach, Wiegard (2002).

51 Vgl. hierzu auch Wissenschaftlicher Beirat beim BMWi (1998), S. 29, insbesondere Anmerkung Nr. 7. 
bietet. Sofern es gelänge, die Schuld in endlicher Zeit zu tilgen, argumentiert Townley (1981), sei ein pareto-effizienter Übergang möglich.

Für eine kleine offene Volkswirtschaft können aber Spremann (1984) bzw. in einer Erweiterung Breyer (1989) zeigen, dass es einen solchen Weg nicht geben kann. Da der Renditevorsprung eines KDV ausschließlich durch die Verzinsung der Rentenbeiträge begründet wird, und sich die Staatsschuld in jeder Periode um eben diesen Zinssatz vergrößert, bleibt die Staatsschuld pro Kopf konstant. Ökonomisch ist dieser Sachverhalt eindeutig. ${ }^{52} \mathrm{Da}$ die Beiträge zum ursprünglichen UV lohnunabhängig sind, werden hierdurch keine Verzerrungen auf dem Arbeitsmarkt induziert. Das Umlageverfahren beinhaltet somit keine Ineffizienzen, die durch einen Wechsel zu einem KDV gemindert oder beseitigt werden können.

Wird hingegen ein Rentensystem mit lohnproportionalen Beiträgen und Pauschalrenten in einer kleinen offenen Volkswirtschaft betrachtet, dann induziert die Erhebung der Rentenbeiträge eine Verzerrung der Arbeitsangebotsentscheidung, da diese wie Lohnsteuern aufgefasst werden. Die Umstellung auf ein KDV bedeutet nun, dass die ursprünglichen Rentenbeiträge zum UV durch nichtverzerrendes Sparen, hier durch Beiträge an das KDV, substituiert werden. Die Erwerbstätigen in der Umstellungsperiode erfahren folglich einen Nutzengewinn, der besteuert werden kann, ohne das Nutzenniveau des ursprünglichen UV zu unterschreiten. Da dieses auch für die nachfolgenden Generationen gilt, kann Homburg (1990) nachweisen, dass die für Kompensationsleistungen in der Umstellungsperiode aufgenommene Schuld in endlicher Zeit getilgt werden kann. Sofern also ein (netto)lohnelastisches Arbeitsangebot vorausgesetzt wird, bestehen im ursprünglichen Umlageverfahren Ineffizienzen, die durch einen Wechsel zum KDV zunächst gemindert und letztlich beseitigt werden. Für den Fall einer Pauschalrente sind die hierdurch erreichten Nutzengewinne so groß, dass ein pareto-effizienter Übergang gelingt. Die Höhe der Elastizität ist dabei, wie Brunner (1994) und Fenge, Schwager (1995) in einem Modell mit unterschiedlichen Individuen aufzeigen, von entscheidender Bedeutung. Nur dann, wenn die induzierten Verzerrungen groß oder die Unterschiede zwischen den Individuen sehr klein sind, reichen die Nutzengewinne für eine Kompensation der Übergangsgeneration aus.

Die Annahme einer Pauschalrente besitzt in Bezug auf den Sachverhalt in der gesetzlichen Rentenversicherung wenig empirische Relevanz, da die Rentenhöhe hier im Wesentlichen proportional zu den Beitragsleistungen ist. D.h. die 
GRV bietet teilhabeäquivalente Renten. Ausgehend von einem solchen Umlageverfahren kann Fenge (1995 bzw. 1997) den Nachweis erbringen, dass ein pareto-effizienter Systemwechsel unmöglich ist. In einem Rentensystem mit lohnabhängigen Beiträgen und einer Pauschalrente ist mit Homburg (1990) bekannt, dass die gesamte Beitragsbelastung als Steuer zu interpretieren ist und daher verzerrende Wirkungen auf die Arbeitsangebotsentscheidung hervorruft. Werden nun teilhabeäquivalente Renten betrachtet, dann ist der Teil der Beitragsleistung, der bei einer Verzinsung in Höhe des realen Kapitalmarktzinses zu der Rente führt, die dem Individuum in einem Umlageverfahren zuteil wird, frei von allokativen Verzerrungen. Lediglich der Anteil der Beitragsleistung, dem keine Rentenansprüche gegenüberstehen, wird als Steuer interpretiert. M.a.W. in einem Rentensystem mit Teilhabeäquivalenz reduziert sich der implizite Steueranteil. $^{53}$

\subsection{Zusammenfassung}

In der wissenschaftlichen Diskussion herrscht weitgehende Einigkeit darüber, dass ein Kapitaldeckungsverfahren eine i.d.R. höhere interne Rendite als ein Umlageverfahren bietet. Insofern erscheint ein KDV einem UV überlegen und ein Systemwechsel wünschenswert. Ausgehend von einem etablierten Umlageverfahren gelingt ein pareto-effizienter Wechsel allerdings nur in sehr einfachen Modellen mit exogenem Arbeitsangebot und pauschalen Rentenbeiträgen und -leistungen oder bei Annahme eines stark (netto)-lohnelastischen Arbeitsangebotes in einer Kombination mit lohnproportionalen Beiträgen und Pauschalrenten. Die ökonomische Begründung hierfür ist im Wesentlichen trivial. Werden ausschließlich Pauschalrenten geleistet, so kann der Rentenbeitrag vollständig als Steuer aufgefasst werden, die entsprechende Verzerrungen auf dem Arbeitsmarkt begründet. Die Substitution der Rentenbeiträge bzw. Steuern in einem UV durch Sparleistungen, d.h. nicht verzerrende Beitragsleistungen in einem $\mathrm{KDV}$, begründet dann Effizienzgewinne.

Werden hingegen Systeme mit teilhabeäquivalenten Rentenzahlungen betrachtet, d.h. die Rentenhöhe steht in einem proportionalen Verhältnis zur Höhe der geleisteten Beiträge, dann kann nur ein Teil der Rentenbeiträge eines Umlageverfahrens als Steuer interpretiert werden. Sofern die Grundvoraussetzung, eine relativ zum Zinssatz schrumpfende Ökonomie, zutrifft, dann verursacht nur der Teil des Rentenbeitrages eines teilhabeäquivalenten Umlageverfahrens, dem 
keine Rentenleistungen gegenüberstehen, Verzerrungen auf dem Arbeitsmarkt. Der Rest ist als Versicherungsprämie für die Arbeitsangebotsentscheidung ohne Belang. Die durch einen Wechsel zu erzielenden Effizienzgewinne sind folglich kleiner als im Fall mit Pauschalrenten und reichen unter Ausschluss von Kopfsteuern nicht aus, um die eingangs aufgenommene Staatsschuld zu tilgen.

In Bezug auf die Umstellung der gesetzlichen Rentenversicherung auf eine Bürgerrente mit ergänzender, privater Kapitaldeckung folgt daraus zunächst, dass es im Zuge eines Systemwechsels - wie intuitiv zu erwarten war - Verlierer geben wird, welche die implizite Schuld eines Umlageverfahrens zu tragen haben. Zudem wird die Abkehr von dem im Grundsatz teilhabeäquivalenten System der gesetzlichen Rentenversicherung negative Wirkungen auf den Arbeitsmarkt induzieren.

Fraglich bleibt hingegen das Ausmaß dieser Effekte. Hierzu ist Folgendes festzustellen:

Die Verteilung von Entlastungen und Belastungen ist grundsätzlich die Aufgabe der Politik. Die Durchbrechung des Paretokriteriums, ein im Übrigen sehr restriktiver Bewertungsmaßstab, kann daher für politische Entscheidungsprozesse nicht als Ausschlussgrund verstanden werden. Wesentlich wichtiger sind hier die genauen Wirkungen und die Intensitäten. Buslei, Kraus (1996, S. 74) argumentieren beispielsweise, dass sich eine Gesellschaft durchaus dafür aussprechen kann, temporäre Mehrbelastungen zu akzeptieren, wenn diese nur moderat sind und die zu erwartenden Vorteile sehr hoch ausfallen und nach Möglichkeit in zeitlicher Nähe zu realisieren sind.

Die Wirkungen auf den Arbeitsmarkt sind hingegen nicht auszuräumen, da die Umstellung auf ein Grundrentenmodell eine implizite Erhöhung der Lohnsteuern begründet. Während in einem teilhabeäquivalenten UV die Beiträge nur teilweise als Steuern zu verstehen sind, kann in einer Bürgerrente der gesamte Betrag als Lohnsteuer aufgefasst werden. Entscheidend ist auch hier das Ausmaß. Wenngleich eine jegliche quantitative Abschätzung aufgrund der vorhandenen Unsicherheit über zukünftige Rentenleistungen und der enormen Detailfülle des Rentenrechts nicht gelingt, ist festzuhalten, dass der implizite Steueranteil der GRV-Beiträge, der in dem Renditeunterschied zum Ausdruck kommt (Vgl. dicke, durchgezogene Linie in Abbildung 1, S. 29), ebenfalls allokative Verschlechterungen hervorruft. Während dieser für die heutige Rentnergeneration nur rund ein Drittel der Beitragsleistung ausmacht, beträgt der 
implizite Steueranteil für die im Jahr 2003 ins Erwerbsleben Eintretenden rund 50 v.H. ${ }^{54}$ Zudem ist, wie Börsch-Supan (1998c) anmerkt, zu beachten, dass die Teilhabeäquivalenz der gesetzlichen Rentenversicherung nicht allen Versicherten klar und verständlich ist bzw. dass viele nicht an diese „glauben“. Es ist daher zu vermuten, dass die verzerrenden Wirkungen im bestehenden System der Rentenversicherung größer sind, als dies der Steueranteil erwarten lässt.

Kurzum, auch ein Umlageverfahren beinhaltet negative Wirkungen auf den Arbeitsmarkt, die sich infolge eines weiter steigenden impliziten Steueranteils in Form von Schwarzarbeit oder anderen Ausweichreaktionen ausweiten werden. ${ }^{55}$ Zwar lassen sich hierdurch die zusätzlichen Verzerrungen einer Bürgerrente nicht relativieren, allerdings erscheint eine detaillierte Analyse der Vorund Nachteile einer Systemveränderung durchaus sinnvoll. Im folgenden Abschnitt wird daher zunächst die Frage der Kosten eines solchen Systems diskutiert.

54 Vgl. hierzu Wissenschaftlicher Beirat beim BMWi (1998), S. 23 und insbesondere Abbildung 3 .

Zur Vermeidung von Missverständnissen sei betont, dass der Wissenschaftlicher Beirat beim BMWi (1998, S. 24) zwar ein KDV für vorziehenswürdig erachtet aber aufgrund der Altansprüche der GRV einen vollständigen Systemwechsel ablehnt. Ferner wird eine Einführung eines Grundrentenmodells nach Biedenkopf-Miegel (1997) ausdrücklich nicht befürwortet, da „dies keinen Beitrag zur Lösung der demographisch bedingten Krise der Rentenversicherung und zum Abbau der Fehlanreize leisten würde, die von dieser Versicherung [gemeint ist die GRV] ausgehen" (Wissenschaftlicher Beirat beim BMWi 1998, S. 14). Dieses Urteil bezieht sich allerdings nur auf den obligatorischen Teil eines solchen Rentensystems. Dass die Einführung einer Bürgerrente auch als ein partieller Wechsel zu einem KDV zu werten ist, wird nicht gesondert thematisiert. Eine Einbeziehung dieses Denkansatzes im Zuge einer quantitativen Analyse verschiedener Modelle mit partieller Kapitaldeckung unterbleibt. 


\section{Die Kosten einer Bürgerrente}

\subsection{Vorhandene Berechnungen - Ein Literaturüberblick}

\subsubsection{Vorbemerkungen}

Infolge der langjährigen politischen und wissenschaftlichen Diskussion und der kontinuierlichen Weiterentwicklung der unterschiedlichen Grundrentenmodelle ist ein lückenloser Überblick über die existierenden Berechnungen oder Schätzungen der zu erwartenden Rentenausgaben in dem System der BiedenkopfMiegelschen Bürgerrente nur schwer zu geben. Wenngleich die Frage der Kostenwirkungen eines Grundrentenmodells in der Literatur oftmals aufgegriffen worden ist, ist die Anzahl der quantitativen Analysen gering. Die folgenden Abschnitte bieten einen Überblick über die wichtigsten Berechnungen eines derartigen Rentensystems.

\subsubsection{Schmähl}

In einer Fortschreibung eines Berechungsansatzes zur Bestimmung der Zusatzkosten in einer Sockelrente (vgl. Schmähl 1974), d.h. einer bedarfsunabhängigen Mindestrente als Sicherungsinstrument innerhalb der bestehenden Alterssicherungssysteme, schätzt Schmähl (1988) die zusätzlichen Belastungen, die durch die Einführung einer Grundrente nach Miegel, Wahl (1985) induziert werden. In einer Abwandlung der vorgestellten Biedenkopf-Miegelschen Bürgerrente garantiert dieses Grundrentenkonzept eine Leistungshöhe von lediglich 40 v.H. des durchschnittlichen Nettoarbeitsentgeltes (rund $€ 405$ pro Monat) ab einem Verrentungsalter von 63 Jahren.

Unter der Annahme einer im Zeitfenster von 1968 bis 1985 proportional zur „Zuwachsrate des durchschnittlichen Nettoarbeitsentgelts“56 der abhängig Beschäftigten wachsenden Ökonomie ergäbe sich für das Jahr 1985 ein zusätzlichen Finanzbedarf in Höhe von $€ 10,2$ Mrd. $^{57}$ Die von Schmähl (1988) erwarteten Gesamtkosten einer Grundrente betragen somit rund $€ 88,6 \mathrm{Mrd}^{58}$ oder rund 9,5 v.H. des BIP.

\footnotetext{
56

Schmähl (1988), S. 299.

Vgl. Schmähl (1988), S. 299.

Der Betrag ergibt sich aus den von Schmähl (1988) genannten $€ 10,2$ Mrd. und den Ausgaben der GRV für Rentenzahlungen im Jahr 1985 in Höhe von € 78,3 Mrd. Quelle: VDR (2000), S. 221.
} 
Schmähl kann hierdurch den Mehraufwand einer Grundrente quantitativ für ein Jahr bestimmen. Die Berechnungsmethode ist allerdings kritisch zu betrachten, da eine Fortschreibung von Modellergebnissen, die im Jahr 1968 bei der Untersuchung eines Sockelrentenmodells erzielt worden sind, zur Bestimmung der Mehrkosten eines Bürgerrentenmodells im Jahr 1985 wenig beitragen kann.

\subsubsection{Buttler, Jäger, Rappl}

Auch Buttler et al. (1987) präsentieren eine Berechnung zur Abschätzung der Kosten bei Einführung einer Grundsicherung, die in Anlehnung an Miegel, Wahl (1985) ausgestaltet ist. Hierbei handelt es sich um ein Modell mit konstanten Preisen (Stand 1990) und ohne Berücksichtigung von realem Wachstum oder von Strukturveränderungen auf der Grundlage von Makrodaten.

Zur Vereinfachung begrenzen Buttler et al. den Kreis der Anspruchsberechtigten auf gesetzlich Rentenversicherte, weil Daten zur Ermittlung der Rentenanwartschaften der Selbständigen, Freiberufler oder der Beamten nur unzulänglich verfügbar seien. ${ }^{59}$ Zudem wird angenommen, dass alle Personen, die eine GRV-Rente beziehen, Anspruch auf eine Bürgerrente besitzen, d.h. es werden im Wesentlichen die Regelungen der gesetzlichen Rentenversicherung übernommen. Da keine Überführungsphase vorgesehen ist, werden die Aufstokkungsbeträge zum Stichtag der Systemumstellung am 1. Januar 1990 in voller Höhe fällig.

Die Höhe der erforderlichen Aufstockungsbeträge bestimmen Buttler et al. dadurch, dass ausschließlich die durchschnittlichen Ansprüche der Einfachrentner, d.h. Personen mit nur einer Rente (z.B. eine Altersrente), auf das Grundsicherungsniveau aufgestockt werden. Bei Mehrfachrentnern erfolgt hingegen keine Aufstockung.

Hierdurch ergäben sich im Umstellungsjahr bei einem Leistungsniveau in Höhe von alternativ $€ 409$ oder $€ 511$ pro Monat (rund 32,5 v.H. bzw. 40,6 v.H. des durchschnittlichen Volkseinkommens pro Kopf) Gesamtkosten in Höhe von $€ 108,4$ Mrd. bzw. $€ 116,6$ Mrd. Im Vergleich zu einer Beibehaltung der gesetzlichen Rentenversicherung wäre dies ein Mehrbetrag in Höhe von $€ 14,8 \mathrm{Mrd}$. beziehungsweise $€ 23,0 \mathrm{Mrd}$. Infolge eines sukzessiven Abschmelzens der Altansprüche zeigen Buttler et al., dass eine Grundrente in Höhe von 32,5 v.H. des Volkseinkommens pro Kopf ab dem Jahr 2010, d.h. 20 Jahre nach der Umstellung, zu Einsparungen führt. Eine Grundrente in Höhe von

$59 \quad$ Buttler, Jäger, Rappl (1987), S. 749. 
40,6 v.H. des Volkseinkommens pro Kopf führe hingegen dauerhaft zu höheren Ausgaben als eine Fortführung der GRV.

Buttler et al. zeigen, dass die Umstellung des Rentensystems zu Beginn mit erheblichen Mehrbelastungen verbunden ist. Deutlich wird aber auch, dass im Falle eines geringen Leistungsniveaus Kostenvorteile innerhalb der ersten 20 Jahre zu erwarten sind. Kritisch zu bewerten ist hingegen das sehr pauschale Modell zur Vermeidung von Leistungskumulationen. Da Buttler et al. lediglich Einfachrenten bis zum Grundsicherungsbetrag aufstocken, wird implizit angenommen, dass Personen, die mehrere Renten (z.B. aufgrund eigenständiger Berufstätigkeit und wegen des Todes eines Angehörigen) beziehen, einen Gesamtanspruch in Höhe des Grundsicherungsbetrages erreichen. Hierzu sei angemerkt, dass 10,9 v.H. (17,0 v.H.) aller weiblichen und rund 7,6 v.H. (11,0 v.H.) aller männlichen Doppelrentner mit der Kombination aus Versichertenund Witwenrente in den alten Bundesländern einen Gesamtzahlbetrag von 32,5 v.H. (40,6 v.H.) des durchschnittlichen Volkseinkommens pro Kopf der Wohnbevölkerung im Jahr 1999 nicht erreichen ${ }^{60}$.

Das von Buttler et al. angewendete Verfahren führt somit vermutlich zu Unterschätzungen der tatsächlichen Aufstockungsbeträge.

Wenngleich eine ex-post Betrachtung einer geleisteten Prognosearbeit nicht üblich ist, sei an dieser Stelle ein Vergleich erlaubt. Die Gesamtausgaben der gesetzlichen Rentenversicherung werden für das Jahr 1990 auf rund $€ 94$ Mrd. und für das Jahr 2000 auf $€ 102$ Mrd. geschätzt. Gegenüber den in den alten Bundesländern tatsächlich angefallenen $€ 110,1 \mathrm{Mrd}$. bzw. $€ 173,1 \mathrm{Mrd}$. beinhaltet die Prognose eine erhebliche Kostenunterschätzung, so dass die Validität der erzielten Prognoseergebnisse zumindest fraglich erscheint. ${ }^{61}$

\subsubsection{Müller, Tautz}

Eine „grobe Abschätzung “62 der Überführungskosten einer in Anlehnung an Miegel, Wahl (1985) konzipierten Grundrente bieten Müller, Tautz (1996). Die Berechnungen konzentrieren sich im Wesentlichen auf die alten Bundesländer

60

Quelle: VDR (Lfd. Jahre), hier (2000), S. 257. Der Vergleich erfolgt zum Jahr 1999, weil die VDR-Statistik umgestellt worden ist. Die Antelle für die neuen Bundesländer betragen bei Frauen 1,2 v.H. bzw. 3,0 v.H. und bei Männern 0,3 v.H. bzw. 0,7 v.H. Quelle: VDR (2003).

62 Vgl. Müller, Tautz (1996), S. 771. 
und auf eine Umstellung der gesetzlichen Rentenversicherung. Mittels einer proportionalen Hochrechnung der gesetzlich Versicherten auf die Gesamtanzahl der potentiellen Leistungsbezieher in einer Grundrente werden auch die Gruppen der Selbständigen und der Freiberufler sowie der nicht gesetzlich Versicherten mit Ausnahme der Beamten in die Prognose einbezogen. Ausgeschlossen bleiben folglich Personen in den neuen Bundesländern ${ }^{63}$ und Versorgungsempfänger des öffentlichen Dienstes. Die Bestimmung der Kosten der Hinterbliebenenversorgung erfolgt in der Grundrente pauschalisiert, indem die zu erwartenden Aufwendungen an Witwen bzw. Witwer unterhalb einer Altersgrenze von 63 Lebensjahren zur Hälfte als Kostenfaktor in einer Bürgerrente gewertet werden. ${ }^{64}$ Zur Fortschreibung der Rentenausgaben in der GRV werden Modelle des VDR zur Vorausberechnung der zu erwartenden Beitragssätze verwendet. Die Annahmen zur Demographie und zur Ökonomie folgen hierbei dem Gutachten des Sozialbeirates zum Rentenversicherungsbericht 1996 (Sozialbeirat 1996). Die Umstellung wird für das Jahr 2000 angenommen.

Bei einer Leistungshöhe der Grundsicherung von 40 v.H. des durchschnittlichen Nettoarbeitsentgeltes aller abhängig Beschäftigten betrage die monatliche Zahlung rund $€ 614$. Für ein allgemeines Verrentungsalter von 63 Lebensjahren folge hieraus ein Gesamtaufwand für Rentenausgaben in Höhe von rund $€ 147$ Mrd. Da die Rentenausgaben der GRV in den alten Bundesländern auf $€ 131$ Mrd. geschätzt werden, führt der Systemwechsel zu deutlichen Kostensteigerungen. ${ }^{65}$ Begründet seien die Mehrbelastungen durch die Aufstockungsbeträge und die Leistungen an die bislang nicht gesetzlich Rentenversicherten. Sofern konstante Preise angenommen werden, ergäbe sich bereits für das Jahr 2020 ein Kostenvorteil für die Bürgerrente in Höhe von $€$ 4,6 Mrd., der sich bis zum Jahr 2040 auf gut $€ 27$ Mrd. oder rund 15,4 v.H. der GRV-Kosten ausweitet.

Um auch die Wirkungsweisen einer veränderten Finanzierungsart aufzuzeigen, werden zudem hypothetische Beitragssätze für eine Grundrente bestimmt, die je zur Hälfte aus direkten und aus indirekten Steuern (Variante I) bzw. ausschließlich aus indirekten Steuern (Variante II) finanziert werden. Zur Deckung der Kosten werden in der Bürgerrente ausschließlich Einnahmen aus den Beiträgen der Pflichtversicherten berücksichtigt. Nach Müller, Tautz könnten

Wenngleich diese in dem Gesamtmodell zwar enthalten sein mögen, beziehen sich sämtliche Ergebnisse ausschließlich auf die alten Bundesländer.

64 Vgl. Müller, Tautz (1996), S. 777.

Müller, Tautz (1996), S. 776. 
„Pflichtbeiträge als hypothetischer Ersatz für eine Steuerfinanzierung [..] eines Grundrentensystems betrachtet werden" ${ }^{66}$ In der GRV hingegen werden neben den Beiträgen der Pflichtversicherten auch Beiträge aus Lohnersatzleistungen und Bundeszuschuss als Einnahmepositionen berücksichtigt.

Für die Beitragssätze der GRV ergeben sich im Wesentlichen die Ergebnisse des Sozialbeirates (Sozialbeirat 1996), d.h. im Ausgangsjahr 2000 ist ein Beitragssatz in Höhe von gut 20 v.H. zu erwarten, der bis zum Jahr 2030 auf 25,5 v.H. ansteigt und anschließend auf einem annähernd konstanten Niveau verbleibt. In einer Grundrente ist hingegen bereits im Jahr 2000 mit deutlich höheren Beiträgen zu rechnen. In der Variante I ergäben sich 31,2 v.H. und in der Variante II wegen eines einzubeziehenden Kaufkraftausgleiches 35,9 v.H. Infolge eines Abschmelzens der Altansprüche reduzieren sich diese zunächst, so dass im Jahr 2020 rund 29,6 v.H. bzw. 34,5 v.H. zu erwarten seien. Aufgrund der demographischen Veränderungen steigen die Belastungen bis 2030 wieder an und erreichen zum Ende des Prognosehorizontes im Jahr 2040 einen Wert von 30,2 v.H. bzw. 36,2 v.H. M.a.W. die Beitragsbelastungen verbleiben während des gesamten Prognosezeitraumes oberhalb der GRV-Werte.

Müller, Tautz zeigen, dass eine Grundrente bereits ab dem Jahr 2020 zu Kostenvorteilen gegenüber einer Fortführung der gesetzlichen Rentenversicherung führen kann. Die mitunter stark vereinfachenden Annahmen der Modellierung erscheinen aber zumindest fraglich. Als Beispiel hierfür sei die Vorgehensweise zur Einbeziehung der Beamten und Selbständigen oder die Bewertung der Hinterbliebenenversorgung genannt. Wesentliche Wirkungszusammenhänge - z.B. Leistungskumulationen - werden dadurch nicht ausreichend berücksichtigt.

Trotz der ausgewiesenen Kostenvorteile einer Bürgerrente erwarten Müller, Tautz, dass die Beiträge in einer Bürgerrente unabhängig von der Finanzierungsart durchgängig über den entsprechenden GRV-Beiträgen lägen. Dies ist zumindest für die Variante I, die paritätisch aus direkten und indirekten Steuern finanziert wird und in der im Unterschied zur Variante II kein Kaufkraftausgleich zu berücksichtigen ist, ein überraschendes Ergebnis. Die Nichtübereinstimmung der Ergebnisse beider Untersuchungsteile ist vermutlich durch die Definition der Beitragsbemessungsgrundlage eines Rentensystems begründet. Während sich die Einnahmen einer Grundrente nach Müller, Tautz ausschließ-

66 Müller, Tautz (1996), S. 780. 
lich aus Pflichtbeiträgen zusammensetzen, ergeben sich die Einnahmen der GRV aus der Summe von Pflichtbeiträgen, Beiträgen aus Lohnersatzleistungen und aus dem Bundeszuschuss ${ }^{67}$. Hieraus folgt eine deutlich „schmalere Basis ${ }^{668}$ für die Einnahmen einer Bürgerrente. Obwohl Müller, Tautz auch auf der Ausgabenseite erhebliche Veränderungen vornehmen und die Lohnsteuerquote in einer Bürgerrente reduzieren, wird dieser Effekt offenbar nicht kompensiert.

\subsubsection{Besendorfer, Borgmann, Raffelhüschen}

Auf der Grundlage einer Generationenbilanzierung untersuchen Besendorfer et al. (1998) neben den inter- und intragenerationalen Verteilungswirkungen die zu erwartende Entwicklung des Rentenbeitrages und des Versorgungsniveaus in einer Bürgerrente nach Biedenkopf-Miegel (1997). Im Unterschied zu den bereits vorgestellten Studien wird im Rahmen des „Generational Accounting“ von der Existenz einer intertemporalen Budgetrestriktion ausgegangen. D.h. eine verringerte Belastung einer Generation führt zwangsläufig zu einer erhöhten Belastung einer anderen Generation. Für ein umlagefinanziertes Rentensystem folgt hieraus (c.p.) ein direkter Zusammenhang zwischen der Höhe der Beitragssätze und dem Versorgungsniveau der Rentner der selben Periode.

Besendorfer et al. unterstellen einen Systemwechsel im Jahr 1996 und bestimmen die zu erwartenden Beiträge bzw. das zu erwartende Versorgungsniveau für ein Leistungsniveau in Höhe von 55 v.H. des Volkseinkommens pro Kopf (rund $€ 787$ pro Monat im Basisjahr). Rechtsstand der GRV ist in der Untersuchung alternativ der Status vor bzw. nach Einarbeitung der durch das Rentenreformgesetz 1999 (RRG'99) induzierten Veränderungen.

Es zeigt sich, dass die Belastung der Erwerbstätigen infolge des im Vergleich zur GRV ausgeweiteten Kreises der Anspruchsberechtigten kurzfristig leicht über das Niveau beider Varianten der gesetzlichen Rentenversicherung ansteigt. Bereits ab dem Jahr 2005 bzw. ab 2007 (d.h. rund neun bzw. elf Jahre nach der Umstellung) führe eine Bürgerrente hingegen zu geringeren Belastungen. In einer langfristigen Perspektive vergrößerten sich die Vorteile einer Bürgerente gegenüber der GRV vor bzw. nach Einwirkung des RRG'99 erheb-

67 Angemerkt sei hier, dass der Anteil des Bundeszuschusses im Jahr 1996 an den Gesamteinnahmen der GRV rund 21 v.H. beträgt. Unter Berücksichtigung der besonderen Bundeszuschüsse ergibt sich für das Jahr 2000 ein Anteil in Höhe von gut 23 v.H. (Quelle: VDR 2003).

Vgl. Müller, Tautz (1996), S. 780. 
lich und betrügen im Jahr 2040 rund 8,5 bzw. 6,2 Prozentpunkte. ${ }^{69}$ Gleichwohl merken Besendorfer et al. an, dass dies vornehmlich durch das vergleichsweise geringere Versorgungsniveau einer Bürgerrente begründet sei. Zwar läge das Nettorentenniveau infolge der Aufrechterhaltung der bereits erworbenen Ansprüche bis 2010 ungefähr auf dem Niveau der GRV in Höhe von 70 v.H falle aber dann annähernd linear $a b$ und erreiche langfristig einen Wert in Höhe von 55 v.H. ${ }^{70}$

Kurzum, Besendorfer et al. bestätigen im Wesentlichen die Ergebnisse der vorherigen Untersuchungen. Bereits nach einer mittelfristigen Zeitspanne, hier nach rund zehn Jahren, sind spürbare Entlastungen zu erwarten. Die angeführte Begründung für die Kostenvorteile, ein im Vergleich zu GRV verringertes Nettorentenniveau, ist jedoch nur in Bezug auf den Rechtsstand vor dem RRG 1999 sachlich richtig. Während Besendorfer et al. hier ein annähernd konstantes Versorgungsniveau in Höhe von rund 70 v.H. bis zum Jahr 2040 berechnen, zeigen die Autoren auch, dass dieses Niveau bei Anwendung des RRG'99 ebenfalls sinkt. Die Berücksichtigung eines Demographiefaktors in der Rentenformel führt nämlich dazu, dass sich das Nettorentenniveau zunächst stärker als in einer Bürgerrente verringert und erst ab 2030 eine höhere Absicherung bietet. $^{71}$

\subsubsection{Burgert, Dicke, Glismann, Horn ${ }^{72}$}

Burgert et al. (1999) präsentieren ein Modell zur Abschätzung der Kostenbelastungen in einem Übergangszenario auf der Grundlage von Makrodaten, welches durch die Annahme konstanter Preise sowie einer Nichtberücksichtigung realen Wachstums und realer Strukturveränderungen gekennzeichnet ist.

Die Umstellung der Rentensysteme erfolgt innerhalb einer langfristigen (25 Jahre) Überführungsphase und ist weitestgehend entsprechend der Biedenkopf-Miegelschen Regeln ausgestaltet. $\mathrm{Zu}$ beachten ist hierbei, dass Burgert et al. das allgemeine Verrentungsalter in der Bürgerrente in Anlehnung an ein durchschnittliches Renteneintrittsalter der Alters- und Erwerbsminderungsrentner in den bestehenden Systemen auf zunächst 59 Jahre gesenkt haben. Im

\footnotetext{
69 Vgl. Besendorfer, Borgmann, Raffelhüschen (1998), S. 13.

70 Besendorfer, Borgmann, Raffelhüschen (1998), S. 12.

71 Vgl. Besendorfer, Borgmann, Raffelhüschen (1998), Abb. 2, S. 10 und Abb. 3, S. 12.

72 Vgl. Burgert, Dicke, Glismann, Horn (1999).
} 
Verlauf der Prognose steigt dieses dann bis 2020 auf bis zu 62 Lebensjahre an. Im Vergleich zum Biedenkopf-Miegelschen Originalentwurf wird damit der gesicherte Personenkreis deutlich ausgeweitet. Bei einer Bürgerrente in Höhe von 52,5 v.H. des Volkseinkommens pro Kopf der Wohnbevölkerung (monatlich rund $€ 787^{73}$ pro Person) ergäben sich bei einer Umstellung zum 01. Januar $2000^{74}$ zunächst Mehrbelastungen. Im Jahr 2010 betrügen diese rund 0,7 v.H. bis 1,3 v.H. des Bruttoinlandsproduktes ${ }^{75}$. Infolge des Abschmelzens der Altansprüche verringere sich der Abstand zu den bestehenden Systemen sukzessive, so dass Burgert et al. für die Bürgerrente zum Prognosehorizont im Jahr 2040 Kostenvorteile von bis zu 1,4 v.H. des BIP erwarten.

Kurzum, Burgert et al. weisen in ihrer Modellierung die grundsätzliche Finanzierbarkeit eines Systemwechsels bei Wahrung der bislang erreichten Rentenansprüchen quantitativ nach. Sie können zeigen, dass die Umstellung der Alterssicherungssysteme auf eine Bürgerrente langfristig Kostenvorteile verspricht. Infolge des gewählten Modelldesigns sind Vergleiche zum Kontrollszenario, einer Fortführung der bestehenden Systeme, nur stichprobenartig zu bestimmten Zeitpunkten (hier in den Jahren 2000, 2010 und 2040) möglich. Hierdurch bleiben jedoch wesentliche Fragen, beispielsweise die Höhe der Mehrbelastungen am Anfang der Umstellungsphase oder die Entwicklung der Mehrbelastungen im Zeitablauf, im Unklaren.

\subsubsection{Miegel, Wahl ${ }^{76}$}

Miegel, Wahl (1999) betrachten ein eingeführtes System einer Bürgerrente und den Prozess der Systemüberführung in einem Modell mit konstanten Preisen sowie unter Ausschluss realen Pro-Kopf-Wachstums und Strukturveränderungen. Hierbei handelt es sich um ein Modell, in dem in einer Abwandlung des Biedenkopf-Miegelschen Originalvorschlages eine Hinterbliebenenversorgung

73 Vgl. Burgert, Dicke, Glismann, Horn (1999), S. 18. Angenommen wird eine jährliche Leistung von DM 18840 (rund $€$ 9449) im Jahr 2000. Dies entspricht einer monatlichen Zahlung in Höhe von $€ 787$.

74 Vgl. Burgert, Dicke, Glismann, Horn (1999), S. 2 und 19. In der Studie selbst bleibt offen, ob das als Umstellungsjahr 1995 oder 2000 angenommen worden ist. Mit Miegel, Wahl (1999, S. 157) wird klar, dass mit Ausnahme der Demographie die Strukturdaten und Preise des Jahres 1995 übernommen worden sind.

75 Im Vergleich zu den nach Prognos (1998) zu erwartenden Ausgaben der bestehenden Systeme (Bürgerente: Variante I, Prognos: altes Recht, oberes und unteres Wirtschaftsszenario). Vgl. Burgert, Dicke, Glismann, Horn (1999), insbesondere S. 13 ff. Vgl. Miegel, Wahl (1999). 
nur für Waisen und Erzieher von Waisen gegeben ist. Eheleute sind nicht mehr wechselseitig über das obligatorische Rentensystem abgesichert, d.h. sie erhalten im Todesfall des Ehepartners keine Leistungen. ${ }^{77}$

Wird zunächst die Problematik des Systemwechsels ausgeblendet, und ein eingeführtes System einer Bürgerrente betrachtet, dann ergibt sich Folgendes: Bei einem Leistungsniveau in Höhe von 52,5 v.H. des Volkseinkommens pro Kopf (d.h. monatlich rund $€ 818$ brutto im Jahr 2000) und einem auf 62 Jahre fixierten Verrentungsalter betragen die Aufwendungen für eine Bürgerrente im Jahr 2000 rund $€ 152,0 \mathrm{Mrd}^{78}$ Hiervon sind rund $€ 148,6 \mathrm{Mrd}$. durch die Zahlungen an die Personen in einem Alter von 62 oder mehr Lebensjahren erklärt. Der verbleibende Differenzbetrag wird zur Begleichung der Ansprüche aufgrund einer Erwerbsminderung oder eines Todes eines Angehörigen (hier also für Leistungen an Waisen sowie Erzieher von Waisen) angesetzt. Wie sich die Höhe dieses Pauschalbetrages ableitet, bleibt unklar. Da Miegel, Wahl (1999) die Aufwendungen für die bestehenden Systeme der Alterssicherung mit rund Mrd. $€ 210$ beziffern, bedeutet ein eingeführtes System einer Bürgerrente im Jahr 2000 einen Kostenvorteil in Höhe von rund $€ 52$ Mrd. (d.h. rund 2,5 v.H. des angenommenen BIP). Bis zum Jahr 2040 weiten sich die Einsparvolumina deutlich aus, so dass diese gegenüber einer Beibehaltung des "Status quo" rund $€ 89$ Mrd. $^{79}$ betrügen (d.h. rund 10,2 v.H. des BIP).

Für den Fall einer Systemüberführung präsentieren Miegel, Wahl ein Zahlenwerk, welches auf der Grundlage der Modellierungen Burgert et al. (vgl. Abschnitt 3.1.6) entstanden ist. Im Unterschied zu den dort getroffenen Annahmen wird das allgemeine Verrentungsalter im Jahr 2000 auf 60 Lebensjahre festgesetzt und in Preisen des Jahres 2000 gerechnet. ${ }^{80}$ Die Grundtendenz der Projektion ist daher identisch: Bereits im Jahr 2010 seien Kostenvorteile zu erwarten. Während die Aufwendungen für die bestehenden System auf rund $€ 256,3$ Mrd. geschätzt werden, erfordere eine Bürgerrente nur $€ 246,4$ Mrd. Das entspricht einem Kostenvorteil in Höhe von 0,5 v.H. des Bruttoinlandspro-

77 Vgl. Miegel, Wahl (1999), S.121. Begründet wird dies von Prof. Dr. Miegel auf Anfrage mit dem anderen Gesellschaftsbild, welches in einem Rentensystem mit individualisierten Leistungsansprüchen zu Ausdruck kommt. Anders hingegen in einem älteren Vorschlag für eine Grundsicherung. Vgl. hierzu Miegel, Wahl (1985), S. 66f.

Vgl. Miegel, Wahl (1999), S. 132.

Vgl. Miegel, Wahl (1999), S. 132.

Vgl. Miegel, Wahl (1999), S. 144 und 157. 
duktes. Aufgrund des Abschmelzens der Altansprüche vergrößere sich dieser Vorteil im Prognoseverlauf und erreiche im Jahr 2040 rund 4 v.H. des BIP.

In den Berechnungen von Miegel, Wahl (1999) wird aufgezeigt, dass ein eingeführtes System einer Bürgerrente erheblich günstiger als die bestehenden Systeme ist. Die Kostenvorteile betragen in den Jahren 2000 rund 2,5 v.H. des Bruttoinlandsproduktes und erweitern sich bis zum Jahr 2040 auf rund 10,0 v.H. Die Aussagefähigkeit der Berechnungen bleibt jedoch aufgrund einer Beschränkung der Hinterbliebenenversorgung auf die Sicherung der Waisen sowie deren Erzieher beschränkt, da eine entsprechende Korrektur der Aufwendungen für die bestehenden Systeme nicht vorgenommen wird.

Welche Auswirkungen die Einführung der Bürgerrente auf den einzelnen Berechtigten haben wird, ist auf Grundlage der Quelle kaum oder nicht zu übersehen. Insbesondere bleibt die zentrale Fragestellung ungeklärt, inwieweit eine Lebensstandardsicherung der Individuen auch in einer Bürgerrente gewährleistet ist.

\subsubsection{Zusammenfassung}

\subsubsection{Wesentliche Ergebnisse der Modellrechnungen}

Trotz oder gerade wegen der langjährigen Diskussion um ein Grundrentensystem sind die vorgestellten Analysen nur schwer miteinander vergleichbar. Begründet ist dies in den gewählten Vereinfachungen, hier insbesondere die Definition des zu sichernden Personenkreises (alle Bürger oder nur gesetzlich Versicherte) oder des zu versichernden individuellen Risikos (Alter, Erwerbsminderung, Tod eines Angehörigen). Die resultierenden Kostenschätzungen differieren dementsprechend stark. Eine Übersicht über die erzielten Ergebnisse und die wesentlichen Eckpunkte der betrachteten Rentensysteme bietet Tabelle 2 (Seite 49f.).

Hierbei zeigt sich das erwartete Bild. Während Biedenkopf, Miegel (1997) bei einem Leistungsniveau in Höhe von 55 v.H. des Volkseinkommens pro Kopf die Mehrbelastungen zu Beginn der Umstellungsphase auf rund 2 v.H. der bisherigen Ausgaben taxieren, erwarten beispielsweise Schmähl (1988) und Buttler et al. (1987) trotz eines geringeren Leistungsniveaus (40 v.H. des durchschnittlichen Einkommens aus unselbständiger Tätigkeit) Mehrkosten in Höhe von 11,6 v.H. bzw. 25 v.H. Ähnliches gilt auch in Bezug auf die weitere Kostenentwicklung einer Bürgerrente. Während Burgert et al. (1999) und Miegel, Wahl (1999) bei einem Leistungsniveau in Höhe von 52,5 v.H. des Volkseinkommens bereits nach rund zehn Jahren Kostenvorteile in Aussicht stellen, 
werden diese von Besendorfer et al. (1998) für Leistungen in Höhe von 55 v.H. erst nach rund 20 Jahren erwartet. Im Unterschied hierzu sind mit Buttler et al. (1987) für derartig hohe Leistungen keine Vorteile gegenüber einer Fortführung der bestehenden Systeme zu erreichen.

Wird trotz der bestehenden Unterschiede zwischen den Modellen der Versuch unternommen, die wesentlichen Eckpunkte der Analysen zusammenzufassen, lässt sich mit Einschränkungen Folgendes festhalten:

Die Einführung einer Bürgerrente ist infolge der Aufstockung kleiner Renten auf einen Mindestbetrag zunächst mit Mehrbelastungen verbunden. Mittelfristig hingegen sind die Kosten und damit die Belastung für die Erwerbstätigen geringer als bei einer Fortführung der bestehenden Systeme. Eine exakte Quantifizierung der Höhe und der Dauer der zu erwartenden Mehrbelastungen erscheint aber auf der Grundlage der bestehenden Berechnungen nicht möglich.

\subsubsection{Implikationen der Modellgestaltung}

Neben einer Detailbetrachtung ist auch eine grundsätzliche Kritik zu formulieren, die alle genannten Studien betrifft. Wenngleich die Hochrechnung der zu erwartenden Kosten für ein Rentensystem mit pro Kopf einheitlichen Leistungen trivial erscheint, bereitet die Ermittlung der Anzahl der Anspruchsberechtigten auf der Grundlage der Statistiken der Rentenversicherungsträger erhebliche Schwierigkeiten. Begründet ist dies darin, dass die Statistiken i.d.R. Rentenfälle, nicht aber Rentner ausweisen und eine Vielzahl der Rentner mehrere Leistungen einer oder verschiedener Trägerschaften beziehen. Da eine Bürgerrente grundsätzlich individualisierte Leistungen gewährt und ein Mehrfachbezug auch bei Erfüllung mehrerer Anspruchsgrundlagen ausgeschlossen ist, besteht die Schwierigkeit folglich in dem Rückschluss auf die Anzahl der Rentner. Auf der Grundlage von Makrodaten ist dies nur bedingt möglich und erfolgt beispielsweise bei Buttler et al. (1987) oder Müller, Tautz (1996) durch Vereinfachungen und Pauschalisierungen.

Als Beleg für die Bedeutung der Leistungskumulationen in den bestehenden Rentensystemen sei der folgende Exkurs erlaubt. Hierbei wird zwischen systeminternen und -externen Kumulationen, d.h. dem gleichzeitigen Empfang gleich- oder verschiedenartiger Leistungen einer oder mehrerer Trägerschaften differenziert. Die Betrachtung systeminterner Leistungstiberschneidungen beschränkt sich hierbei auf die gesetzlichen Rentenversicherung. 


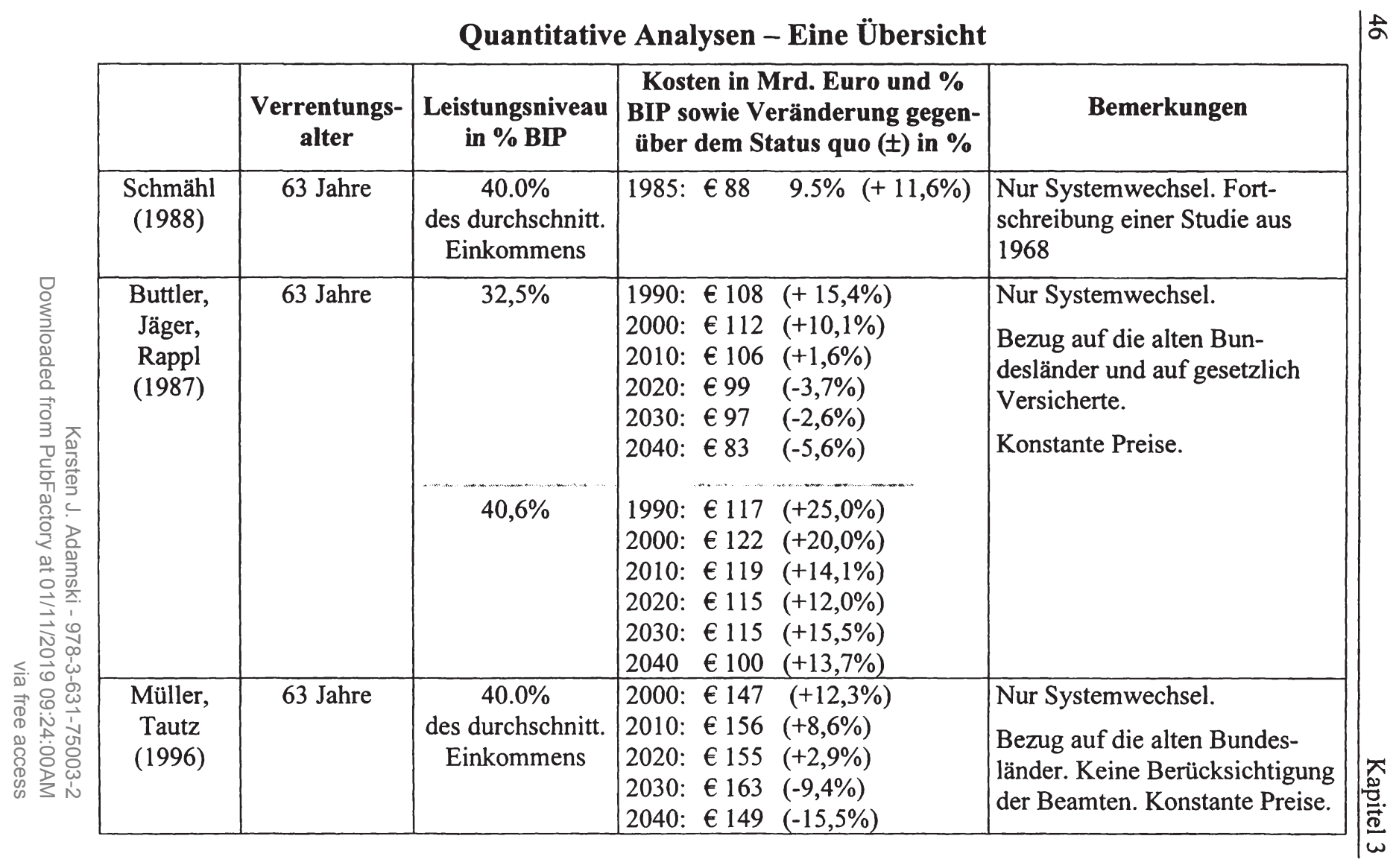




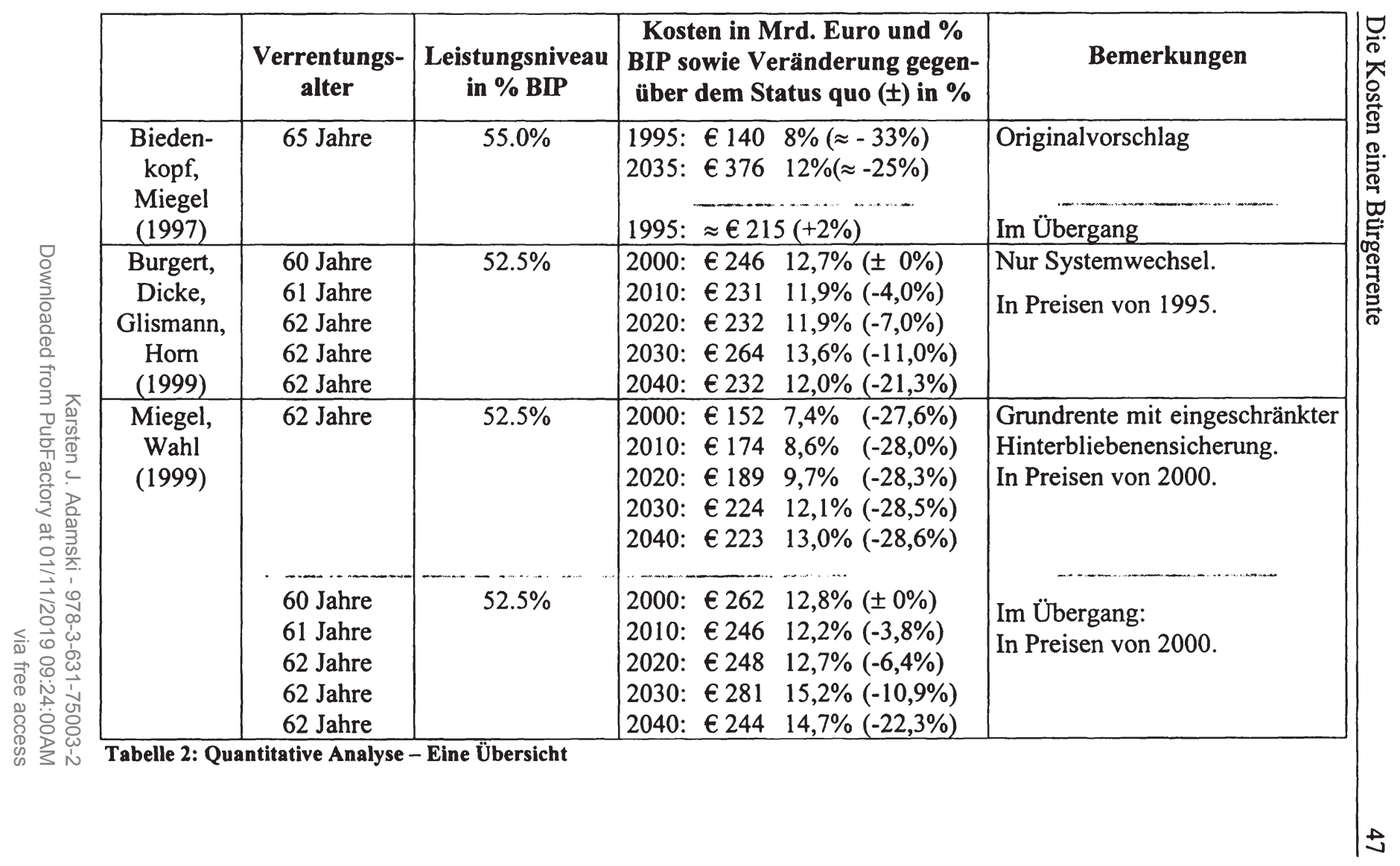




\section{Systeminterne Kumulationen am Beispiel der gesetzlichen Renten-} versicherung

In der gesetzlichen Rentenversicherung bezogen am 01. Juli 1999 rund 18,5 v.H. aller Rentner oder 3.444.885 Versicherte (davon 93 v.H. Frauen) mehr als eine Rente. Der durchschnittliche Gesamtrentenzahlbetrag beträgt rund $€ 984$ bei Frauen und $€ 1117$ bei Männern, der sich aus Leistungen aufgrund eigenständiger Erwerbstätigkeit und aus der Hinterbliebenenversorgung zusammensetzt. Von dem Gesamtbetrag entfallen bei Frauen rund 57 v.H. auf die Hinterbliebenenversorgung. Bei Männern ist dieser Anteil mit rund 16 v.H. erheblich geringer. ${ }^{81}$ Die Bedeutung der Hinterbliebenensicherung ist folglich stark geschlechtsspezifisch geprägt.

\section{Systeminterne Leistungskumulationen in der GRV}

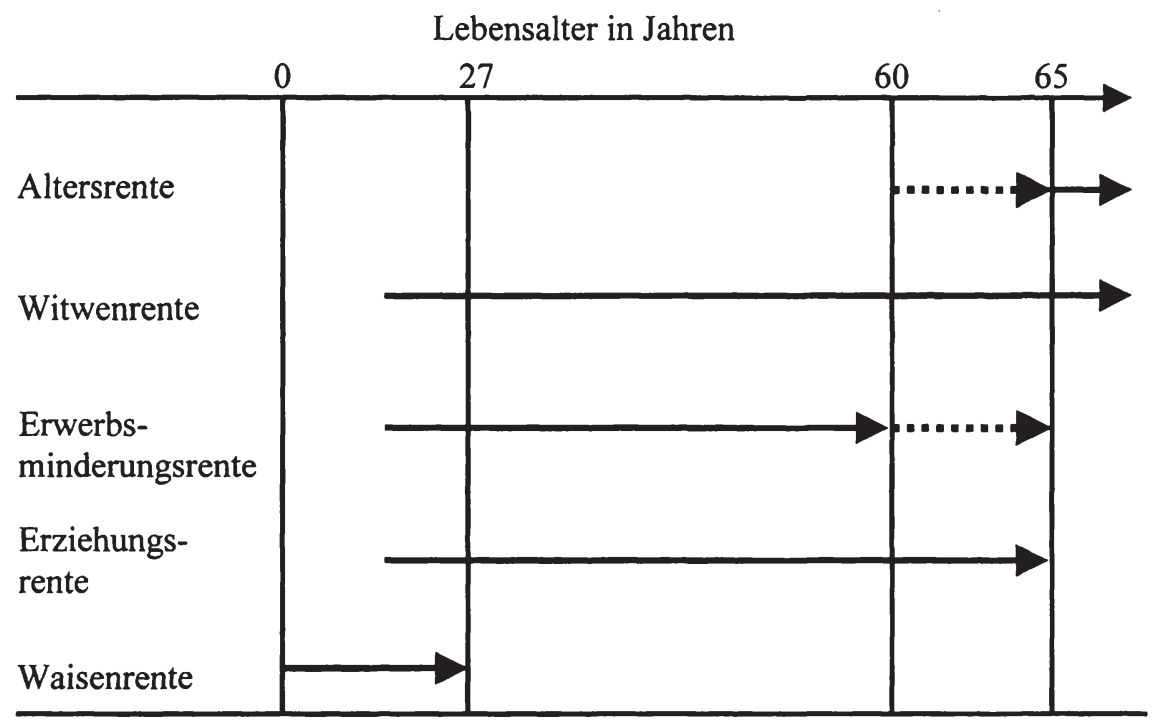

Abbildung 2: Systeminterne Leistungskumulationen in der GRV

81 Quelle: VDR (Lfd. Jahre), hier (2000), S. 244. 
Die Möglichkeiten eines Zusammentreffens verschiedener Leistungsarten ergeben sich aus der Abbildung 2, in welcher das Leben einer Person schematisch auf einem Zeitstrahl aufgetragen ist. Die Möglichkeiten zum Bezug einer Leistung der gesetzlichen Rentenversicherung, z.B. einer Regelaltersrente, sind entsprechend der gesetzlichen Rahmenbedingungen eingezeichnet. ${ }^{82}$

Bis zum vollendeten 60. Lebensjahr bestehen die Möglichkeiten für einen mehrfachen Rentenbezug nur im Falle einer Kombination aus einer Erwerbsminderungsrente und einer Rente wegen Todes eines Angehörigen, d.h. einer Witwen-, Erziehungs- oder bis zum vollendeten 27. Lebensjahr einer Waisenrente ${ }^{83}$.

Danach, d.h. bis vor Vollendung des 65. Lebensjahres, können Mehrfachrenten bei einem Zusammentreffen von Alters- oder Erwerbminderungsrenten ${ }^{84}$ und Renten wegen Todes vorkommen. Nach Erreichen der Regelaltersgrenze reduzieren sich die Kumulationsmöglichkeiten. Sie bestehen dann nur beim gleichzeitigen Bezug einer Alters- und einer Witwen- bzw. Witwerrente.

\section{Systemexterne Kumulationen}

Die Möglichkeiten zur Kombination von Leistungen verschiedener Einrichtungen des Sozialsystems sind weitreichend, da im Verlauf eines Erwerbslebens Anwartschaften in mehreren Rentensystemen erworben werden können. Beispielsweise beziehen im Jahr 1999 rund 12 Millionen Renten- oder Pensionsempfänger $\mathrm{ab}$ dem vollendetem 65. Lebensjahr insgesamt 18,9 Millionen Leistungen aus Alterssicherungssystemen, d.h. jeder Leistungsempfänger erhält im Durchschnitt rund 1,6 Leistungen. ${ }^{85}$ Auf systemübergreifende Kumu-

82 Die Darstellung bezieht sich auf eine Altersrente gemäß $\S 35$ bis $\S 38$ sowie $\S 40$ und $\S 236$ bis $\S 237$ a sowie Anlagen 19 bis 22 , für eine Erwerbsminderungsrente gemäß $\S 43$ und $\S 45$ sowie $\S 240$, für eine Witwenrente $\S 46$ und $\S 242 \mathrm{a}$ bis $\S 243 \mathrm{a}$, für eine Erziehungsrente gemäß $\S 47$ und für eine Waisenrente nach $\S 48$ SGB VI. Die Abbildung konzentriert sich auf Grundsätzlichkeiten. Weitestgehend unberücksichtigt bleiben die Regelungen für das Zusammentreffen von Renten mit Einkommen oder Einkommensäquivalenten zu berücksichtigen. Hierzu siehe $\S 89 \mathrm{ff}$. SGB VI.

83 Unbeachtet bleiben hier die Bestimmungen nach $\S 48$ (5).

84 Der gleichzeitige Bezug einer Alters- und einer Erwerbsminderungsrente ist ausgeschlossen. Vgl. § 89 SGB VI.

Vgl. Deutsche Bundesregierung (2001b), S. 81. Nicht berücksichtigt sind reine KLGLeistungen. 
lationen gleich- oder verschiedenartiger Leistungen entfallen in den alten Bundesländern dabei rund 28 v.H. ${ }^{86}$

Die wesentlichen Kumulationsformen bei Ansprüchen aufgrund eigenständiger Erwerbstätigkeit sind in Abbildung 3 dargestellt. Im Einzelnen sind die Überschneidungen von Leistungen der gesetzlichen Rentenversicherung (GRV), der Beamtenversorgung (BAV), der Berufsständischen Versorgungssysteme (BSV), der Alterssicherung der Landwirte (AdL), der Betrieblichen Altersversorgung (BV) und der Zusatzversorgung im öffentlichen Dienst (ZOED) wiedergegeben.

Es fällt auf, dass wesentliche Anteile der systemübergreifenden Kumulationen durch ein Zusammentreffen von Leistungen primärer und sekundärer Sicherungssysteme begründet werden. Beispielsweise beziehen 17 v.H. der über 65-Jährigen, die eigenständige Rentenansprüche in der GRV besitzen, Renten aus Systemen der betrieblichen Altersversorgung. Ähnliches gilt auch für die AdL-Versicherten, die zumeist auch über gesetzliche Renten verfügen können.

Für einen Systemübergang sind hingegen Kumulationen zwischen Systemen wichtiger, die im Modellschema der Weltbank zur ersten Säule der Alterssicherung zählen, weil diese durch eine Bürgerrente substituiert werden. Verstehen wir die BAV, BSV und die ZOED und mit Einschränkungen auch die AdL als Systeme der ersten Sicherungssäule, dann zeigt sich, dass der überwiegende Anteil der Personen ab 65 Jahren in diesen Systemen zugleich eine gesetzliche Rente bezieht.

Kurzum, die bestehenden Verflechtungen innerhalb des Leistungsspektrums der Alterssicherungssysteme sind aufgrund der nicht unerheblichen Wirkungen auf die Höhe der erforderlichen Aufstockungsbeträge in einer quantitativen Analyse einer Bürgerrente zu berücksichtigen. Da dies auf der Grundlage der zugänglichen Makrodaten, z.B. der Statistiken der einzelnen Versicherungsträger, nicht gelingt, bieten die bislang erstellten Studien nur sehr grobe Anhaltspunkte bezüglich der zu erwartenden Kosten oder Lasten eines Grundrentensystems.

Unmittelbare Schlüsse auf die Realität oder daraus abzuleitende Politikempfehlungen sind auf der Grundlage dieser Modellierungen nur sehr eingeschränkt möglich.

86 Systemexterne Kumulationen erfordern eine Systemvielfalt. Da diese Voraussetzung für die Gruppe der über 64-Jährigen in den neuen Bundesländern nicht gegeben war, sind dort mit Stand 1999 keine nennenswerten systemexternen Kumulationen vorhanden. 


\section{Systemexterne Kumulationen eigenständiger Leistungsansprüche}

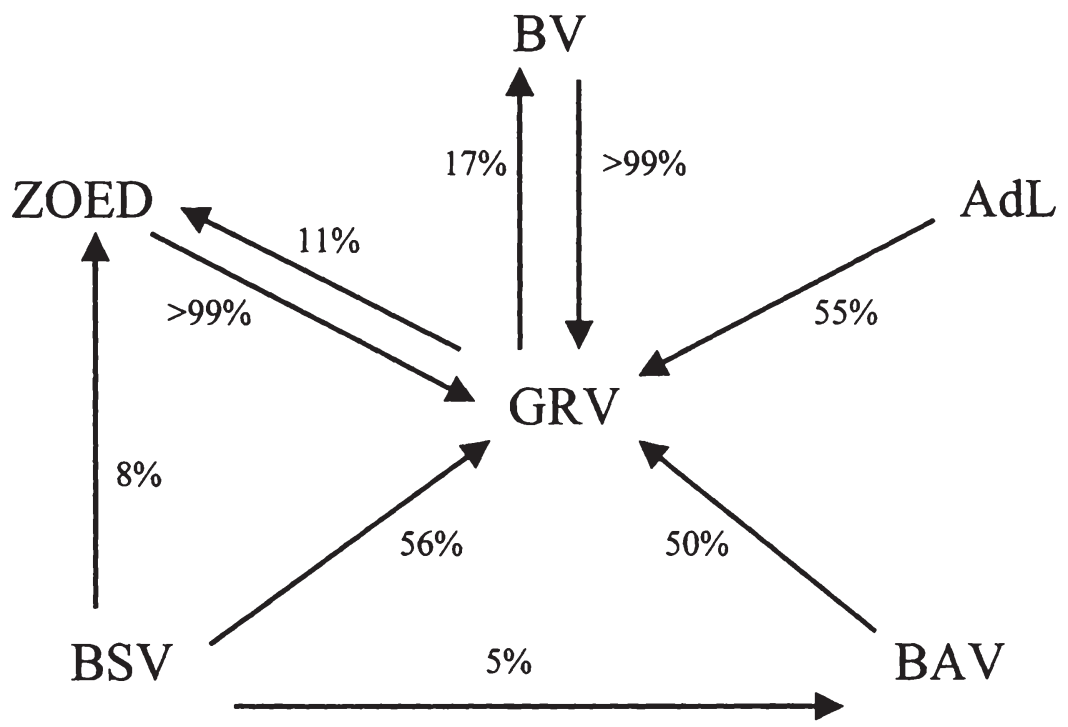

AdL : Alterssicherung der Landwirte

BAV :Beamtenversorgung

BV : Betriebliche Altersversorgung

BSV :Berufsständische Versorgungssysteme

GRV : Gesetzliche Rentenversicherung

ZOED :Zusatzversorgung im öffentlichen Dienst

Abbildung 3: Systemexterne Leistungskumulationen aufgrund eigener Ansprüche in den alten Bundeslăndern ${ }^{87}$.

Als ein Lösungsansatz, die bestehenden Leistungskumulationen zu berücksichtigen, ohne zugleich die Analyse auf bestimmte Personengruppen, z.B. die der gesetzlich Versicherten beschränken zu müssen, wird in dieser Arbeit ein grundsätzlich neuer Ansatz zur Analyse eines Grundrentensystems verwendet:

Ausgehend von der Individualebene, d.h. den Rentenansprüche einer einzelnen Person, wird durch eine Aggregation auf die resultierenden Wirkungen auf der

87 Quelle: Deutsche Bundesregierung (2001b), S. 146. Dargestellt sind Leistungskumulationen, die 5 v.H. oder mehr Personen mit Ansprüchen gegen ein Rentensystem betreffen. 
Makroebene geschlossen. Als Datengrundlage dient hierfür im Wesentlichen das Sozioökonomische Panel des Deutschen Instituts für Wirtschaftsforschung in Berlin.

\subsection{Kosten einer Bürgerrente: Eine neue Berechnung auf der Grundlage von Mikrodaten}

\subsubsection{Vorbemerkungen}

Die Identifikation der Anspruchsberechtigten und im weiteren Analyseverlauf die Aufstellung individueller Rentenkonten erfordert eine umfassende Datenquelle, die eine repräsentative Untersuchung ermöglicht. Der spezifische Charakter der erforderlichen Daten und die strikt mikroökonomische Herangehensweise lassen hierfür insbesondere Paneldaten geeignet erscheinen. Aufgrund der langen Laufzeit und der gezeigten Kontinuität des Paneldesigns, welches eine eingehende Analyse sozialer, ökonomischer und biographischer Inhalte sowie individueller Einstellungen erlaubt, stützt sich diese Arbeit im Wesentlichen auf die Daten des sozioökonomischen Panels (SOEP) des Deutschen Instituts für Wirtschaftsforschung in Berlin. ${ }^{88}$

Hierbei handelt es sich um eine seit 1984 durchgeführte jährliche, repräsentative Wiederholungsbefragung privater Haushalte in Deutschland. Die Stichprobe umfasst im Erhebungsjahr 2001 etwa 12.000 Haushalte mit mehr als 22.000 Personen. Die Inklusionswahrscheinlichkeit beträgt also rund 1/4000. Damit ist das SOEP bei der Erhebung individueller Merkmale führend. ${ }^{89}$ Die Schwerpunkte der Befragung, u.a. Erwerbs- und Familienbiographien sowie Einkommensverläufe, decken sich dabei mit den in dieser Arbeit erforderlichen Informationen.

Die quantitative Erfassung der Bürgerrente wird dabei zunächst auf der Grundlage gesicherter Daten im Sinne einer "Counterfactual Simulation“ für die Jahre 1995 bis 2001 durchgeführt. Hierbei wird unterstellt, dass eine Bürgerrente bereits implementiert worden sei. Dieses Verfahren ermöglicht einen unmittelbaren Vergleich zu den tatsächlich angefallenen und den simulierten Kosten eines Rentensystems. Hierzu ist im Folgenden zunächst ein umfangreicher Begriffsapparat einzuführen.

\footnotetext{
88 Vgl. SOEP Group (2001a).

89 Zum Vergleich: Mikrozensus 1/100; Allbus 1/21500. Quelle: Statistisches Bundesamt (Ifd. Jahre B), S. 9; Koch, Wasmer, Harkness, Scholz (2001), S. 7.
} 


\subsubsection{Die Modelldarstellung}

Die bestehenden Systeme der Alterssicherung werden mit $B S$ und eine Bürgerrente mit $B R$ bezeichnet. Die Gesamtkosten $\mathrm{TK}_{t}^{R}$ eines Rentensystems $R \in\{B S, B R\}$ in einem Jahr $t$ ergeben sich im Wesentlichen aus den Ausgaben für Rentenzahlungen $K_{t}^{R}$, d.h. aus der Summe der individuellen Leistungen $\mathrm{PE}_{B E_{t}^{R}, t}^{R}$ aller Leistungsempfänger $B E_{t}^{R}$ im Jahr $t$. Leistungsempfänger sind Altersrentner und Berechtigte, die wegen sonstiger Anspruchsgrundlagen, z.B. infolge des Todes eines Angehörigen oder einer Erwerbsminderung Rentenzahlungen erhalten. Neben den Aufwendungen für originäre Alterssicherungssysteme sind auch Ausgaben für weitere Sozialsysteme, die die Funktion einer finanziellen Sicherung (für z.B. ältere Menschen) erfüllen, in die Modellierung zu integrieren, da diese rentenäquivalente Leistungen $\mathrm{K}_{1}^{R A E}$ bieten. Zudem sind Ausgaben für Rehabilitationsmaßnahmen $\mathrm{K}_{t}^{\mathrm{Re}}$ und Verwaltungskosten $\mathrm{K}_{t}^{V_{w}}$ als weitere Kostenfaktoren zu berücksichtigen.

Für die bestehenden Systeme ist die Situation im Wesentlichen eindeutig: Die $\mathrm{K}_{l}^{B S}$ ergeben sich aus den Aufwendungen für Rentenzahlungen der Alterssicherungssysteme, die durch eine Bürgerrente substituiert werden. Dies sind zum Einen die Regelsicherungssysteme der ersten Sicherungssäule, hier die gesetzliche Rentenversicherung (GRV) und die Beamtenversorgung (BAV) und zum Anderen ausgewählte sekundäre Alterssicherungssysteme, hier exemplarisch die Zusatzversorgungssysteme im öffentlichen Dienst. Berücksichtigt werden die Ausgaben der Versorgungsanstalt des Bundes und der Länder (VBL), der Arbeitsgemeinschaft kommunale und kirchliche Altersversorgung (AKA) sowie die Bahnversicherungsanstalt Abteilung B (BVA Abt. B).

Exemplarisch für Systeme mit rentenäquivalenten Leistungen werden die Aufwendungen für laufende Hilfe zum Lebensunterhalt (HLU) und für Hilfe in besonderen Lebenslagen (HBL) nach dem BSHG an Rentner berücksichtigt. Für $\mathrm{K}_{t}^{\mathrm{Re}}$ und $\mathrm{K}_{t}^{V_{w}}$ werden vereinfachend die Werte der gesetzlichen Rentenversicherung übernommen.

Für eine Bürgerrente soll Folgendes gelten: Trotz der zu erwartenden Einsparpotentiale bei einer Reduktion der Systemvielfalt auf ein für alle Bürger obligatorisches Regelsicherungssystem werden die Verwaltungskosten $K_{t}^{V_{w}}$ in gleicher Höhe wie in den bestehenden Systemen angesetzt. Dies gewährleistet, 
dass potenzielle Kostendifferenzen nicht durch unterschiedliche Verwaltungsaufwendungen begründet werden. Die Ausgaben für Rehabilitationsmaßnahmen $\mathrm{K}_{t}^{\mathrm{Re}}$ bleiben ebenfalls aus naheliegenden inhaltlichen Erwägungen unverändert. ${ }^{90}$ Rentenäquivalente Leistungen werden in einer Bürgerrente nur als Hilfe in besonderen Lebenslagen gewährt, da ein Bedarf an Hilfe zum Lebensunterhalt nicht entstehen kann. Der entscheidende Parameter ist somit der Aufwand für Rentenzahlungen $K_{t}^{B R}$, die maßgeblich von der spezifischen Ausgestaltung einer Bürgerrente abhängig sind.

Um die Kostensensitivität einzelner Stellgrößen einer Bürgerrente messen zu können, werden die $K_{t}^{B R}$ in einem umfangreichen Teilmodell mit rund 800 typischen Varianten berechnet. Die Einzelheiten der Modellgestaltung ergeben sich aus den folgenden Teilabschnitten:

- Die Bürgerrente wegen Alters,

- die Bürgerrente wegen Todes,

- die Bürgerrente wegen einer Erwerbsminderung,

- die Leistungshöhe,

- und das lebenslauf- und altersbezogene Territorialprinzip.

Hierdurch wird eine Kostenbetrachtung eines solchen Rentensystems und die Einwirkung einzelner Stellgrößen möglich.

90 Die Einbeziehung dieser Kosten in beiden Rentensystemen erfolgt aus methodischen Gründen. Im weiteren Untersuchungsverlauf werden auf der Grundlage der Gesamtkosten die Beitragssätze in einer Bürgerrente bestimmt. 


\subsubsection{Bürgerrente wegen Alters}

Die Fixierung des Lebensalters, ab dem eine Altersrente bezogen werden kann, ist bereits in den bestehenden Systemen Thema zahlreicher Beiträge gewesen. Wenngleich das Rentenreformgesetz 1999 die Altersschwelle für einen Rentenbezug ohne Leistungskürzungen von z.B. 60 Jahren für Frauen mit langfristigen Übergangsregeln ${ }^{91}$ auf einheitlich 65 Jahre anhebt, mehren sich die Forderungen nach einer weiteren Ausweitung der Lebensarbeitszeit. Straubhaar (2000) oder jüngst die Rürup-Kommission ${ }^{92}$ fordern z.B. eine Anhebung der Altersgrenze auf 67 Jahre. Der finanzpolitische Sprecher der CDU-Bundestagsfraktion, Friedrich Merz, spricht sogar von 70 Jahren. ${ }^{93}$

Um den derzeitigen Sachstand und auch die sich abzeichnende Entwicklung in die Modellierung integrieren zu können, wird die Regelaltersgrenze in einer Bandbreite vom 60. bis zum 70. Lebensjahr variiert.

Da die Bürgerrente das obligatorische Alterssicherungssystem für alle Bürger ist, entspricht die Anzahl der Berechtigten wegen Alters der Anzahl der Personen, die die Regelaltersgrenze, hier zum Beispiel das 65. Lebensjahr, überschritten haben.

\subsubsection{Bürgerrente wegen Todes}

Die Hinterbliebenenversorgung ist in einer Bürgerrente deutlich eingeschränkt. Lediglich Eheleute in einem Abschnitt von zehn Jahren vor Erreichen der Regelaltersgrenze, hier in einem Lebensalter von 55 bis unter 65 Jahren, sind im Versicherungsfall anspruchsberechtigt, sofern eine mindestens fünfjährige Nichterwerbstätigkeit des Hinterbliebenen nachgewiesen werden kann. Der Biedenkopf-Miegelsche Vorschlag ist also durch eine Altersgrenze, ab der eine Hinterbliebenenversorgung grundsätzlich gewährt wird und durch eine begrenzte Einbeziehung der Witwen bzw. Witwer, nämlich der Nichterwerbstätigen, gekennzeichnet (Teilvariante SEL55). Der gesetzliche Rahmen der bestehenden Systeme, in dem grundsätzlich alle Hinterbliebene leistungsberechtigt sind, wird durch die Teilvariante ALL18 beschrieben.

\footnotetext{
91 Vgl. Anlage 20 SGB VI.

92 Vgl. Süddeutsche Zeitung (2003a).

93 Vgl. Süddeutsche Zeitung (2003b).
} 
Die Identifikation der Berechtigten erfolgt auf der Grundlage der Daten zur Einkommenssituation der Hinterbliebenenrentner des SOEP. ${ }^{94} \mathrm{Zu}$ beachten ist hierbei, dass die Daten eine Differenzierung zwischen Waisen- und Witwenbzw. Witwerrenten nicht zulassen. Die ausgewiesene Hinterbliebenenrentnerzahl umfasst daher in Teilen auch Waisenrentner. Da Renten an Waisen oder Halbwaisen ähnlich wie in den bestehenden Systemen bis zur Vollendung des 27. Lebensjahres gewährt werden, ${ }^{95}$ sind die Überschneidungsmöglichkeiten begrenzt.

Die Anzahl der Waisenrentner wird aus dem Mikrozensus des Statistischen Bundesamtes übernommen. ${ }^{96}$

\subsubsection{Bürgerrente wegen einer Erwerbsminderung}

Für eine Erwerbsminderung gilt der derzeit gültige Rechtsstand. Die Anzahl der Anspruchsberechtigten ergibt sich dabei direkt aus dem SOEP. Alle Personen unterhalb der Regelaltersgrenze, bei denen nach amtlicher Feststellung eine Erwerbsminderung oder eine Schwerbehinderung vorliegt und die daher keiner Vollzeitbeschäftigung nachgehen können, werden als anspruchsberechtigt angesehen. ${ }^{97}$

\subsubsection{Die Leistungshöhe}

Die Festlegung einer „richtigen“ oder ,angemessenen“ Höhe einer Bürgerrente ist nicht trivial, da sich hierin Wertevorstellungen einer Gesellschaft widerspiegeln. Der zentrale Aspekt ist hier die Trennlinie zwischen Eigenverantwortlichkeit und Solidarität, die eine Gesellschaft bereit ist, von den „Versicherten“ zu fordern bzw. diesen zu bieten ${ }^{98}$. Wenngleich die einzelnen Vorschläge stark variieren, ${ }^{99}$ herrscht weitgehender Konsens darüber, dass eine Bürgerrente ein soziales Existenzminimum garantieren soll. Beispielsweise fordern Breyer ${ }^{100}$

94 Vgl. SOEP GROUP (2000), Welle P, S. 16.

95 Ungeachtet der Bestimmungen des $\S 48(5)$ SGB VI.

96 Quelle: Statistisches Bundesamt, (Lfd. Jahre b), hier (1999), S. 291. Berücksichtigt sind alle Kinder verwitweter Elternteile, die nicht wieder geheiratet haben und noch im Hause der Eltern wohnen.

97 Vgl. SOEP GROUP, (2000), Welle P, S. 24.

98 Vgl. Hüther (1998), S. 270.

99 Vgl.Tabelle 2, S. 49.

100 Vgl. Breyer (1996), S. 127f. 
und Biedenkopf, Miegel ${ }^{101}$ eine Orientierung an den Sätzen des Bundessozialhilfegesetzes. Während Biedenkopf, Miegel wie auch Wahl ${ }^{102}$ dieses mit einer Wahrung des Subsidiaritätsprinzips begründen, weist Breyer zu Recht darauf hin, dass dies eine gewisse politische Stabilität impliziere. „Eine Grundrente auf dem Niveau der Sozialhilfe kann den Rentnern von den zukünftigen politischen Mehrheiten schwerlich verweigert werden “103. Klanberg, Prinz, die ein „rationales System der Grundsicherung“(104 als ein System mit einer Identität von Grundsicherungs- und Sozialhilfeleistungen charakterisieren, argumentieren, dass nur im Falle einer Leistungsgleichheit der erforderliche Spielraum für eigenständige, private Vorsorge gegeben sei. ${ }^{105}$

Die Leistungshöhe einer Bürgerrente kann nur abgegrenzt werden. Es erfolgt daher eine Variation der Leistungshöhe. Die untere Grenze wird in Anlehnung an die vorherrschende Meinung in der Literatur in Höhe der laufenden Hilfe zum Lebensunterhalt angenommen. Wird der durchschnittliche BSHG-Bedarf eines Alleinstehenden ohne Kinder zu Grunde gelegt, entspricht dies unter Berücksichtigung der in einer Bürgerrente zu entrichtenden Beiträge an die Kranken- und Pflegeversicherung der Rentner ungefähr 50 v.H. des Volkseinkommens pro Kopf oder rund $€ 744$ pro Monat. ${ }^{106}$

Die obere Begrenzung der zu betrachtenden Leistungen ist mit der Einschränkung der Finanzierbarkeit frei wählbar. Sie wird hier auf 65 v.H. des Volkseinkommens begrenzt. Eine Analyse der Kostensensitivität kann also in einer Bandbreite von 50 v.H. bis 65 v.H. des Volkseinkommens pro Kopf der Wohnbevölkerung erfolgen.

101 Vgl. Biedenkopf, Miegel (1997), S. 4.

102 Vgl. Wahl (1995), S. 76.

103 Breyer (1996), S. 127. Eine größere Instabilität sieht hingegen Clausing (1985, S. 484).

104 Klanberg (1988), S. 20.

105 Da eine Bürgerrente für die gesamte Bundesrepublik identisch sei, ergäbe sich nach Neumann ein mitunter erweiterter Bedarf an Hilfe in besonderen Lebenslagen, um auch in Ballungsräumen das soziale Existenzminimum gewährleisten zu können. Vgl. Deutsches Institut für Altersvorsorge (Hrsg.) (1998), S. 77.

Der durchschnittliche BSHG-Bedarf (Regelsatz, Kaltmiete, Heizkosten und periodisierte einmalige Leistungen) betrug zum 01.07.1999 rund $€ 604$. Werden die Beiträge an die $\mathrm{KVdR} / \mathrm{PVdR}$ in Höhe von 15 v.H. berücksichtigt, hat die Bürgerrente ein Nettoeinkommen in Höhe von $€ 710$ oder 47,8 v.H. des Volkseinkommens zu sichern. Quelle: Bundesministerium für Arbeit und Sozialordnung (1999), S. 25. 


\subsubsection{Das lebenslauf- und altersbezogene Territorialprinzip}

Die Bindung des Leistungsanspruches an eine langjährige Steuerpflichtigkeit in der Bundesrepublik Deutschland und damit an einen ständigen Wohnsitz im Inland in der Erwerbsphase und im Leistungsfall bedeutet eine erhebliche Verkleinerung des Kreises der potenziell Berechtigten. Neben einer Wartezeitreglung wird der Leistungsanspruch auch an den Ort des Leistungsbezuges gebunden. Eine vergleichbare Beschränkung existiert in den bestehenden Systemen nicht - auch nicht ansatzweise. Es handelt sich um ein Novum in der Sozialpolitik, welches im Hinblick auf z.B. ein zusammenwachsendes Europa kritisch zu sehen ist. Entsprechend der Koordinierungsvorschrift VO (EWG) 1408/71 darf der Anspruch „nicht deshalb gekürzt, geändert, zum Ruhen gebracht, entzogen oder beschlagnahmt werden, weil der Berechtigte im Gebiet eines anderen Mitgliedstaates [...] wohnt“107. Der Biedenkopf-Miegelsche Ansatz ist also rechtlich zumindest bedenklich.

Ungeachtet dieser Fragestellungen werden hier die finanziellen Wirkungen aufgezeigt. Hierzu werden die Kosten einer Bürgerrente ohne (Variante ALL) beziehungsweise mit (Variante SEL) Berücksichtigung einer langjährigen Steuerpflichtigkeit als Anspruchsvoraussetzung bestimmt. Als Variante (PRO) wird eine Proratisierung der Rentenansprüche zugelassen. In Anlehnung an z.B. das schwedische oder das kanadische Grundrentensystem steigt der Anspruch proportional zur Dauer der Systemzugehörigkeit. Nach z.B. 25 oder mehr Jahren besteht ein voller Anspruch.

Die Berechtigten ergeben sich aus dem SOEP. Da eine uneingeschränkte Steuerpflichtigkeit durch einen ständigen Wohnsitz oder zumindest einen gewöhnlichen Aufenthaltsort im Inland begründet wird ${ }^{108}$, ist diese bei in Deutschland geborenen Personen gegeben. Sofern keine temporären Auslandsaufenthalte vorliegen, ergibt sich hieraus die Anzahl der Personen mit einem Anspruch auf den vollen Regelsatz unmittelbar. Die Zahl und die Höhe der zu berücksichtigenden Ansprüche der Immigranten ergeben sich für die Varianten SEL und PRO aus der Aufenthaltsdauer in Deutschland. Die Gesamtanzahl der Leistungsempfänger $B E_{t}^{B R}$ einer Bürgerrente ergibt sich also aus den Personen, die die Vorraussetzungen mindestens einer Anspruchsgrundlage erfüllen. Infolge

107 Vgl. Artikel 10 (1) VO (EWG) 1408/71. Inwieweit die Biedenkopfsche Bürgerrente von der Exportpflichtigkeit nach Artikel 10 a der Verordnung befreit werden kann, ist z. Z. nicht zu übersehen. Vgl. hierzu: Ruland (1999).

Vgl. § $1 \mathrm{EStG}$. 
der differenzierten Betrachtung auf der Grundlage von Mikrodaten können Leistungskumulationen weitgehend vermieden werden. Die Modellierung umfasst die folgenden Variationen:

\section{Bezeichnung der Varianten}

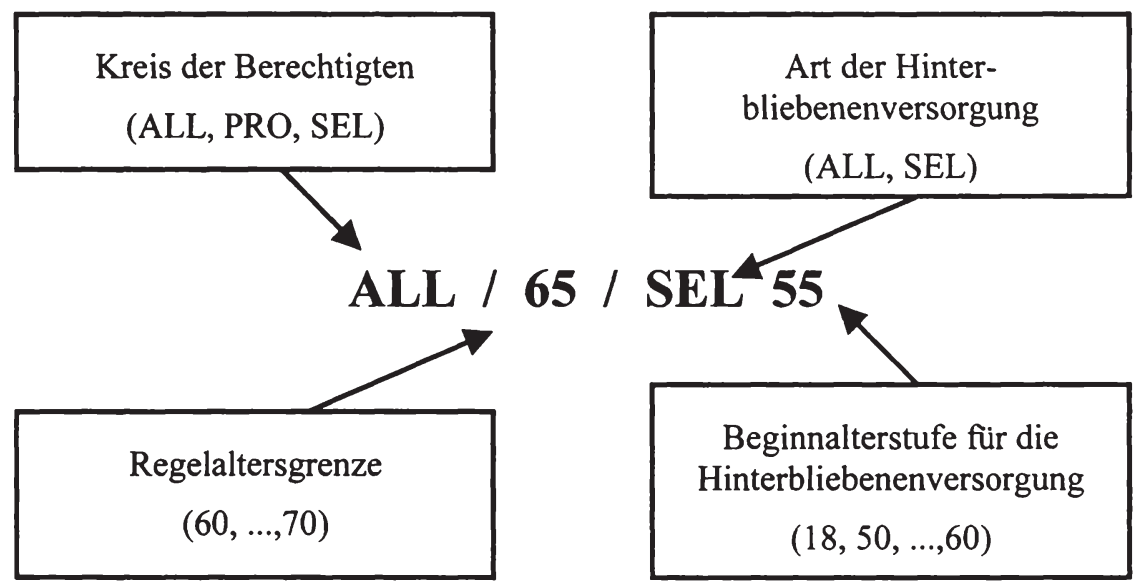

Abbildung 4: Bezeichnung der Varianten

Die exemplarisch dargestellte Form einer Bürgerrente gewährt allen Bürgern einen Leistungsanspruch, sofern diese das 65 . Lebensjahr vollendet bzw. als hinterbliebener Ehegatte das 55. Lebensjahr erreicht haben und zum Zeitpunkt des Leistungsfalles keiner Vollzeiterwerbstätigkeit nachgehen.

Die Biedenkopf-Miegelschen Vorschläge werden in Variante SEL/65/SEL55 abgebildet. Hier ist also der Leistungsanspruch im Unterschied zur Abbildung 4 an eine langjährige Steuerpflichtigkeit gebunden. Der derzeit gültige Rechtsstand der bestehenden Systeme wird in Variante ALL/65/ALL18 auf das System einer Bürgerrente übertragen. Diese Ausgestaltung bietet sich somit insbesondere für vergleichende Betrachtung an und wird als eine Hauptform einer Bürgerrente angesehen. Eine weitere Hauptausgestaltung ist naturgemäß die Variante SEL/65/SEL55. 


\subsubsection{Ein Kostenvergleich: Bestehende Systeme und Bürgerrente}

Mit Hilfe des vorgestellten Modells gelingt es, die hypothetischen Kosten einer Bürgerrente den tatsächlich angefallenen Kosten der bestehenden Systeme in einem Zeitraum von 1995 bis 2001 gegenüberzustellen. Abbildung 5 zeigt die Ergebnisse am Beispiel der weitergehenden Fassung einer Bürgerrente (ALL/ 65/ALL18), in der alle Bürger ab dem 65. Lebensjahr anspruchsberechtigt sind und in der die Hinterbliebenenversorgung wie in den bestehenden Systemen geregelt ist.

\section{Kosten der Rentensysteme im Vergleich}

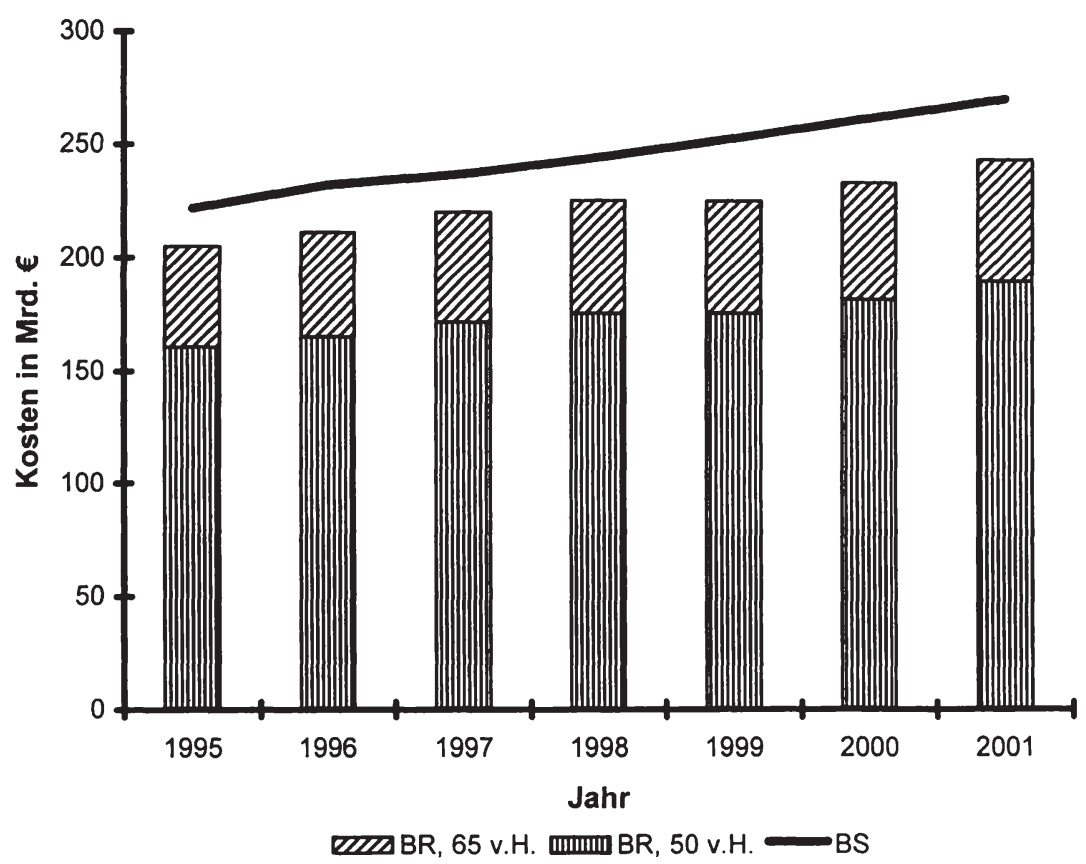

Abbildung 5: Kosten einer Bürgerrente im Vergleich zu den bestehenden Systemen

Die Kosten einer Bürgerrente sind als Balkendiagramm für ein Leistungsniveau in Höhe von 50 v.H. (vertikal gestreifte Balken) bzw. in Höhe von 65 v.H. (vertikal und quer gestreifte Balken) dargestellt. Da es sich im Hinblick 
auf die in der Literatur diskutierten Leistungshöhen um Extrempositionen handelt, wird hierdurch ein Korridor möglicher Kosten angegeben. In der Abbildung entspricht dieser der Länge der quer gestreiften Balken. Die tatsächlich angefallenen Kosten der bestehenden Systeme sind als Liniendarstellung eingezeichnet.

Das Ergebnis ist eindeutig: Anstatt der beispielsweise im Jahr 2001 tatsächlich angefallenen Gesamtkosten in Höhe von rund $€ 270$ Mrd. hätte eine Bürgerrente einen Betrag in Höhe von $€ 189$ Mrd. bis $€ 242$ Mrd. erfordert. Eine eingeführte Bürgerrente wäre also günstiger gewesen. Die Vorteile lägen je nach Leistungsniveau der Bürgerrente bei rund 10 v.H. bis 30 v.H. der tatsächlich angefallenen Kosten.

Wird die Biedenkopf-Miegelsche Fassung einer Bürgerrente (SEL/65/SEL55) betrachtet, sind die Kostenvorteile mit rund 16 v.H. bis 36 v.H. erwartungsgemäß größer.

Die Kostensensitivitäten verschiedener Stellgrößen einer Bürgerrente werden in den nachfolgenden Abschnitten untersucht.

\subsubsection{Die Kostensensitivität einzelner Größen}

Die Gesamtkosten einer Bürgerrente $\mathrm{TK}_{t}^{B R}$ ergeben sich bekanntlich aus der

Summe der Aufwendungen für Rentenzahlungen $K_{t}^{B R}$, Rentenäquivalente $\mathrm{K}_{t}^{R A E}$, Rehabilitationsmaßnahmen $\mathrm{K}_{t}^{\mathrm{Re}}$ und den Verwaltungskosten $\mathrm{K}_{t}^{V_{w}}$. Eine Modifikation der Anspruchsvoraussetzungen wirkt im Wesentlichen ${ }^{109}$ auf $K_{t}^{B R}$, da sich hierin bei einem gegebenen Regelsatz $B R$, und einem definierten Leistungstableau die Anzahl der Leistungsempfänger $B E_{t}^{B R}$ widerspiegelt. Die Veränderung der $B E_{t}^{B R}$ ist also eine Maßgröße zur Beschreibung der Wirkungsweisen einzelner Determinanten.

Ausgangspunkt für die nachfolgende Betrachtung ist die weitergehende Fassung einer Bürgerrente in Form der Variante ALL/65/ALL18.

109 Die Veränderungen der $\mathrm{K}_{t}^{R A E}$ können aufgrund der geringen Wirkungen auf die $\mathrm{TK}_{t}^{B R}$ vernachlässigt werden. 


\subsubsection{Die Regelaltersgrenze}

Die Regelaltersgrenze $a^{*}$ (gemessen in Lebensjahren) besitzt offenkundig einen maßgeblichen Einfluss auf die Anzahl der Anspruchsberechtigten und damit auf die Kosten einer Bürgerrente. Eine Anhebung der Regelaltersgrenze auf $\tilde{a}>a^{*}$ reduziert $B E_{t}^{B R}$ um:

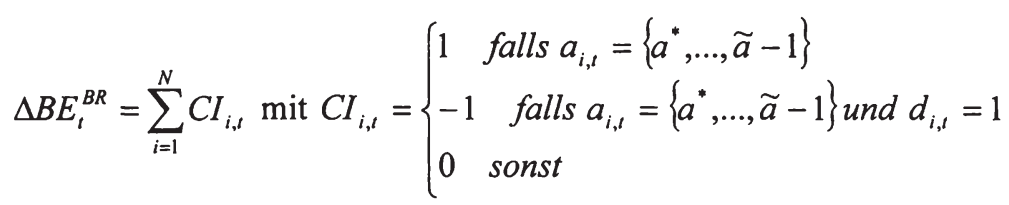

Hierbei bezeichnet $i$ eine beliebige Person, $a_{i, t}$ ihr Alter im Jahr $t$ und $d_{i, t}$ eine Dummyvariable, die den Wert 1 annimmt, wenn $i$ im Jahr $t$ einen Anspruch auf eine Erwerbsminderungsrente bzw. eine Hinterbliebenenrente besitzt. Die Erhöhung der Regelaltersgrenze führt also zu einer Verkleinerung des Personenkreises mit Ansprüchen wegen Alters und zugleich zu einer (begrenzten) Erweiterung des Kreises mit sonstigen Ansprüchen. ${ }^{110}$

Zur Quantifizierung der Kostenwirkungen werden die Gesamtkosten einer Bürgerrente für Regelaltersgrenzen in einer Bandbreite vom 60. bis zum 70 . Lebensjahr in den Jahren 1995 bis 2001 bestimmt und in einem Diagramm aufgetragen. Die Steigung einer Regressionsgeraden, die die Abhängigkeit der Gesamtkosten $\mathrm{TK}_{\imath}^{B R}$ von der gewählten Regelaltersgrenze $a^{*}$ ausdrückt, misst dann den Kosteneffekt einer um ein Jahr verschobenen Regelaltersgrenze. Die Ergebnisse sind in der Tabelle 3 zusammengestellt.

Eine Anhebung der Regelaltersgrenze um ein Lebensjahr bedeutet ausgehend von der Hauptvariante ALL/65/ALL18 im Jahr 1995 eine Verringerung der Gesamtkosten um 2,82 v.H. Im Jahr 2001 sind es 3,54 v.H. Begründet ist dies im Wachstum des Volkseinkommens pro Kopf und des Altenquotienten. Das arithmetisches Mittel ist $-3,11$ v.H. Hierbei ist jedoch zu beachten, dass die Standardabweichung mit 1,58 groß ist.

110 Eine Herabsetzung der Regelaltersgrenze führt analog zu einer Erhöhung der Zahl der Anspruchsberechtigten um:

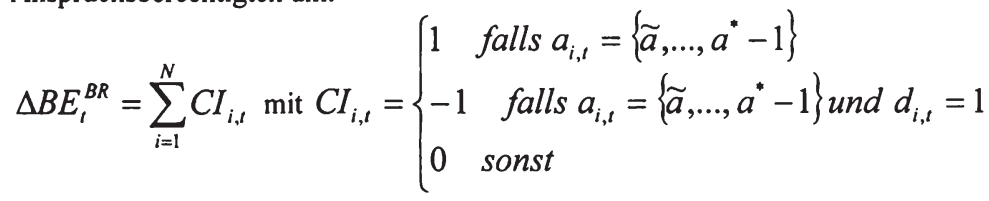


Kostensensitivität einer marginalen Veränderung der Regelaltersgrenze

\begin{tabular}{cccccccc}
\hline & 1995 & 1996 & 1997 & 1998 & 1999 & 2000 & 2001 \\
\hline $\begin{array}{c}\text { Steigung } \\
\text { in \% }\end{array}$ & $-2,82$ & $-2,91$ & $-2,78$ & $-2,99$ & $-3,24$ & $-3,49$ & $-3,54$ \\
$\left(r^{2}\right)$ & $(99,71)$ & $(99,78)$ & $(99,46)$ & $(99,05)$ & $(98,02)$ & $(98,67)$ & $(98,68)$ \\
\hline$\varnothing$ & \multicolumn{7}{c}{$-3,11$} \\
\hline
\end{tabular}

Tabelle 3: Kostensensitivität einer marginalen Veränderung der Regelaltersgrenze.

\subsubsection{Die Einschränkungen bei der Hinterbliebenenversorgung}

Eine Beschränkung des wechselseitigen Leistungsanspruches im Todesfall eines Ehegatten auf Personen, die ein bestimmtes Lebensalter $\tilde{a}<a^{*}$ vollendet oder überschritten haben ${ }^{111}$, besitzt ebenfalls einen maßgeblichen Einfluss auf die Anzahl der Anspruchsberechtigten. Wird das Lebensalter, ab dem grundsätzlich ein Leistungsanspruch gewährt wird, auf $18 \leq \tilde{a}<a^{*}$ angehoben $^{112}$, reduziert sich $B E_{t}^{B R}$ um

$$
\Delta B E_{t}^{B R}=\sum_{i=1}^{N} C I_{i, t} \text { mit } C I_{i, t}=\left\{\begin{array}{ll}
1 & \text { falls } a_{i, t}=(18, \ldots, \tilde{a}-1) \text { und } d_{i, t}=1 \\
0 & \text { sonst }
\end{array} .\right.
$$

Dabei bezeichnet $d_{i, t}$ eine Dummyvariable, die den Wert 1 annimmt, wenn $i$ im Jahr $t$ einen Anspruch auf eine Hinterbliebenenrente besitzt. Die Anzahl der im Leistungsfall Berechtigten wird also deutlich eingeschränkt.

Der resultierende Kosteneffekt wird in Analogie zum vorherigen Teilabschnitt durch die Steigung einer Regressionsgeraden, die die Abhängigkeit der Gesamtkosten $\mathrm{TK}_{t}^{B R}$ von der gewählten Altersgrenze $\widetilde{a}$ misst, ausdrückt. $\widetilde{a}$ ist

111 Biedenkopf, Miegel sprechen von dem 55. Lebensjahr.

112 Zur Abbildung des gegenwärtigen Rechtstandes in der GRV wird angenommen, dass ein wechselseitiger Anspruch auf Hinterbliebenensicherung erst mit der Vollendung 18. Lebensjahres begründet werden kann. 
hierbei in einer Bandbreite vom 18. bis zum 64. Lebensjahr variabel. Tabelle 4 illustriert die Ergebnisse.

\section{Kostensensitivität einer marginalen Veränderung des Startalters der Hinterbliebenensicherung}

\begin{tabular}{cccccccc}
\hline & 1995 & 1996 & 1997 & 1998 & 1999 & 2000 & 2001 \\
\hline $\begin{array}{c}\text { Steigung } \\
\text { in \% } \\
\left(r^{2}\right)\end{array}$ & $-0,11$ & $-0,10$ & $-0,10$ & $-0,09$ & $-0,08$ & $-0,08$ & $-0,09$ \\
\hline$\varnothing$ & $(85,14)$ & $(79,36)$ & $(79,86)$ & $(80,70)$ & $(76,47)$ & $(77,69)$ & $(81,97)$ \\
\hline$\varnothing$
\end{tabular}

Tabelle 4: Kostensensitivität einer marginalen Veränderung des Startalters der Hinterbliebenensicherung

Eine Anhebung des Startalters, ab der ein Ehepaar wechselseitig im Todesfall des Partners abgesichert ist, um ein Lebensjahr bedeutet beispielsweise im Jahr 1995 ausgehend von der Hauptvariante ALL/65/ALL18 eine Verringerung der Gesamtkosten um 0,11 v.H. Im Jahr 2001 sind es 0,09 v.H.

Begründet ist dies vermutlich in Schwankungen der Anzahl der Hinterbliebenenrentner. Der durchschnittliche Kosteneffekt beträgt für die Jahre 1995 bis 2001 rund $-0,09$ v.H. Die Einschränkung der Hinterbliebenensicherung vom Status quo auf die Biedenkopf-Miegelschen Vorschläge reduziert also die Gesamtkosten einer Bürgerrente um rund 3,5 v.H.

In einer weiteren Einschränkung knüpfen Biedenkopf, Miegel (1997) den Leistungsanspruch an die Nicht-Erwerbstätigkeit des Hinterbliebenen zum Zeitpunkt des Leistungsfalles. Zur Abbildung dieser zusätzlichen Anspruchsvoraussetzung nehme die Dummyvariable $d_{i, t}$ in Gleichung (2) dann den Wert eins an, wenn $i$ im Jahr $t$ einen Anspruch auf eine Hinterbliebenenrente besitzt und zugleich nicht vollzeitbeschäftigt ist.

Die Wirkungen auf die Gesamtkosten $\mathrm{TK}_{t}^{B R}$ sind klar: Die weitere Verkleinerung der Anzahl der Anspruchsberechtigten reduziert die Gesamtkosten. Ausgehend von der Variante ALL/65/ALL18 verringert das Erfordernis einer Nichterwerbstätigkeit die Aufwendungen um durchschnittlich 1,12 v.H. Aufgrund einer geringeren Anzahl potenziell berechtigter Hinterbliebenenrentner ist die Wirkung einer um ein Jahr verschobenen Altersgrenze $\tilde{a}$, ab der ein 
wechselseitiger Sicherungsanspruch besteht, kleiner. Der durchschnittliche Kosteneffekt beträgt dann nur $-0,07$ v.H., und die Umstellung des Status quo auf die Biedenkopf-Miegelschen Vorschläge vermindert die Gesamtkosten nur um rund 2,6 v.H.

\subsubsection{Das lebenslauf- und altersbezogene Territorialprinzip}

Die Koppelung des grundsätzlichen Leistungsanspruches an einen ständigen Wohnsitz oder einen gewöhnlichen Aufenthaltsort in der Bundesrepublik Deutschland während Erwerbsphase und im Alter wirkt unmittelbar auf die Anzahl der potenziell Anspruchsberechtigten und damit auf die Kosten einer Bürgerrente. Wird zunächst der Biedenkopf-Miegelsche Ansatz betrachtet, in dem eine Wartezeit $W Z$ formuliert wird, ab der ein Anspruch auf den Bezug der Bürgerrente begründet wird, dann reduziert sich $B E_{t}^{B R}$ um:

$$
\Delta B E_{t}^{B R}=\sum_{i=1}^{N} C I_{i, t} \text { mit } C I_{i, t}= \begin{cases}1 & \text { falls } w z_{i, t}<W Z \text { und } d_{i, t}=1 \\ 0 & \text { sonst }\end{cases}
$$

Hierbei ist $w z_{i, t}$ die individuelle Wartezeit einer Person $i$ im Jahr $t$ und $d_{i, t}$ eine Dummyvariable, die den Wert 1 annimmt, wenn $i$ im Jahr $t$ einen Anspruch auf die Bürgerrente z.B. wegen Alters, einer Erwerbsminderung oder im Falle eines Todes eines Angehörigen besitzt.

Zur Bestimmung der Wirkungen auf die Gesamtkosten werden Wartezeiten von bis zu 24 Jahren betrachtet. Die Steigung einer Regressionsgeraden, die die Abhängigkeit der Gesamtkosten $\mathrm{TK}_{\imath}^{B R}$ von der Wartezeit $W Z$ ausdrückt, misst dann den Kosteneffekt einer um ein Jahr erhöhten Wartezeitregelung.

Eine Ausdehnung der Wartezeit um ein Jahr verringert die Gesamtkosten der Hauptvariante ALL/65/ALL18 beispielsweise im Jahr 1995 um 0,28 v.H. Wird wiederum ein arithmetisches Mittel als Maß zur Beschreibung des durchschnittlichen Kosteneffektes zugelassen, ergeben sich $-0,27$ v.H. Die Umstellung des Status quo auf die Biedenkopf-Miegelsche Fassung einer Bürgerrente, die eine Wartezeit von 24 Jahren vorsieht, führt also zu Einsparungen in Höhe von rund 6,48 v.H. 


\section{Kostensensitivität einer marginalen Veränderung der Wartezeitregelung}

\begin{tabular}{cccccccc}
\hline & 1995 & 1996 & 1997 & 1998 & 1999 & 2000 & 2001 \\
\hline $\begin{array}{l}\text { Steigung } \\
\text { in \% }\end{array}$ & $-0,28$ & $-0,28$ & $-0,27$ & $-0,27$ & $-0,27$ & $-0,24$ & $-0,28$ \\
$\left(r^{2}\right)$ & $(92,87)$ & $(94,28)$ & $(94,27)$ & $(95,24)$ & $(95,46)$ & $(95,23)$ & $(96,07)$ \\
\hline$\varnothing$ & \multicolumn{7}{c}{$-0,27$} \\
\hline
\end{tabular}

Tabelle 5: Kostensensitivität einer marginalen Veränderung der Wartezeitregelung

Wird hingegen eine Proratisierung der Leistungsansprüche zugelassen, sind die Kosteneffekte offenkundig geringer. In diesem Fall reduziert sich die Anzahl der Berechtigten $B E_{t}^{B R}$ um:

$$
\Delta B E_{t}^{B R}=\sum_{i=1}^{N} C I_{i, t} \cdot\left(1-\frac{w z_{i, t}}{W Z}\right) \text { mit } C I_{i, t}= \begin{cases}1 & \text { falls } d_{i, t}=1 \\ 0 & \text { sonst }\end{cases}
$$

Wird also eine z.B. zugewanderte Person $i$ mit $w z_{i, t}<W Z$ betrachtet, ist im Falle einer Proratisierung nur ein anteiliger $\left(\frac{w z_{i, t}}{W Z}\right)$ Bürgerrentenanspruch auszuzahlen, oder genauer: Die Person wird im Zuge der Ermittlung der Anspruchsberechtigten nur anteilig gewertet.

Die Steigung der Regressionsgraden ist folglich flacher. Tabelle 6 zeigt die Ergebnisse für den Fall proratisierter Rentenansprüche.

Eine Ausdehnung der Wartezeit um ein Jahr führt im Falle proratisierter Rentenansprüche im Jahr 1995 zur Einsparungen in Höhe von 0,18 v.H. (Basis sind die Gesamtkosten in der Hauptvariante ALL/65/ALL18). Im Jahr 2001 sind es 0,15 v.H. Als durchschnittlicher Wert der Jahre 1995 bis 2001 ergibt sich ein Effekt in Höhe von $-0,15$ v.H. Wird in Anlehnung an die Biedenkopf-Miegelsche Fassung angenommen, dass ein voller Anspruch nach dem vollendeten 24. Jahr einer Systemzugehörigkeit entsteht, ergeben sich Einsparungen in Höhe von rund 3,6 v.H. 
Kostensensitivität einer marginalen Veränderung der Wartezeitregelung bei einer Proratisierung der Rentenansprüche

\begin{tabular}{cccccccc}
\hline & 1995 & 1996 & 1997 & 1998 & 1999 & 2000 & 2001 \\
\hline $\begin{array}{c}\text { Steigung } \\
\text { in \% }\end{array}$ & $-0,18$ & $-0,17$ & $-0,16$ & $-0,15$ & $-0,14$ & $-0,13$ & $-0,15$ \\
$\left(r^{2}\right)$ & $(97,60)$ & $(98,59)$ & $(98,85)$ & $(98,79)$ & $(98,58)$ & $(99,13)$ & $(99,27)$ \\
\hline$\varnothing$ & & \multicolumn{7}{c}{$-0,15$} & & & \\
\hline
\end{tabular}

Tabelle 6: Kostensensitivität einer marginalen Veränderung der Wartezeitregelung bei einer Proratisierung der Rentenansprüche

\subsubsection{Das Leistungsniveau}

Neben der Anzahl der Bezugsberechtigten besitzt auch das Leistungsniveau $b r_{\text {, }}$ einen erheblichen Einfluss auf die Gesamtkosten. Hierbei ist klar, dass sich die Gesamtkosten $\mathrm{TK}_{t}^{B R}$ annähernd proportional zu $b r$, entwickeln.

\section{Kostensensitivität einer marginalen Veränderung des Leistungsniveaus}

\begin{tabular}{cccccccc}
\hline & 1995 & 1996 & 1997 & 1998 & 1999 & 2000 & 2001 \\
\hline $\begin{array}{l}\text { Steigung } \\
\text { in \% }\end{array}$ & 1,95 & 1,95 & 2,00 & 2,00 & 2,00 & 1,99 & 1,99 \\
$\left(r^{2}\right)$ & $(100)$ & $(100)$ & $(100)$ & $(100)$ & $(100)$ & $(100)$ & $(100)$ \\
\hline$\varnothing$ & & & 1,98 & & & \\
\hline
\end{tabular}

Tabelle 7: Kostensensitivität einer marginalen Veränderung des Leistungsniveaus

Die Wirkungen eines marginal erhöhten Leistungsniveaus auf $\mathrm{TK}_{t}^{B R}$ sind in der Tabelle 7 zusammengestellt. Eine Anhebung des Leistungsniveaus um einen Prozentpunkt steigert die Gesamtkosten um durchschnittlich 1,98 v.H. 


\subsection{Zusammenfassung}

Mit Hilfe eines mikroökonomischen Modells auf der Grundlage des SOEP werden die Kosten einer Bürgerrente und die Wirkungsweisen der zusätzlichen Anspruchsvoraussetzungen des Biedenkopf-Miegelschen Ansatzes untersucht und berechnet. Im Rahmen einer "Counterfactual Simulation“ wird gezeigt, dass eine Bürgerrente zu z.T. erheblichen Kostenvorteilen führen kann.

Werden die grundsätzlichen Regelungen der gesetzlichen Rentenversicherung auch in einer Bürgerrente beibehalten, so dass die gesicherten Personenkreise und die versicherten individuellen Risiken in einer Bürgerrente mit denen in den bestehenden Systemen übereinstimmen (hier in Variante ALL/65/ALL18), betragen die Einsparpotentiale gemessen an den Aufwendungen der bestehenden Systeme je nach Leistungsniveau zwischen 10 v.H. und 30 v.H. Als Leistungsniveau werden dabei 50 v.H. bis 65 v.H. des Volkseinkommens pro Kopf angenommen. In Größen des Jahres 2001 folge hieraus beispielsweise Nettoeinkommen im Ruhestand in Höhe von $€ 655$ bis $€ 852$ pro Kopf.

Werden hingegen die Biedenkopf-Miegelschen Vorschläge umgesetzt, ergeben sich wesentliche Änderungen im Leistungsbereich. Aufgrund einer Verknüpfung des Leistungsanspruches mit einem lebenslauf- und altersbezogenem Territorialprinzip verkleinert sich der Kreis der gesicherten Personen signifikant. Zudem bedingt die Betonung des Subsidiaritätsprinzips im Bereich der Hinterbliebenensicherung, dass auch die gesicherten Risiken nicht in vollem Umfang dem Status quo der bestehenden Systeme entsprechen. Die Kosten eines solchen Rentensystems sind folglich geringer, so dass sich die Einsparpotentiale um rund sechs Prozentpunkte vergrößern.

Wird zur Analyse der Kostenwirkungen einzelner Determinanten einer Bürgerrente die weitergehende Variante zugrundegelegt, ergeben sich die in der Tabelle 8 zusammengestellten Ergebnisse.

Als kostenwirksame Stellgrößen können hierbei im Wesentlichen bekannte Faktoren identifiziert werden: Die Regelaltersgrenze und die Rentenhöhe.

Ausgehend von der Variante ALL/65/ALL18, die den rechtlichen Rahmen der gesetzlichen Rentenversicherung auf eine Bürgerrente überträgt, führt die Erhöhung der Regelaltersgrenze um ein Jahr zu einer Verringerung der Kosten um 3,11 v.H. Die Erhöhung des Leistungsniveaus einer Bürgerrente um einen Prozentpunkt steigert die Kosten um 1,98 v.H. 


\section{Kostenelastizitäten einzelner Determinanten}

\begin{tabular}{|c|c|}
\hline Erhöhung der Regelaltersgrenze um ein Jahr & $-3,11$ v.H. \\
\hline $\begin{array}{l}\text { Erhöhung des Startalters in der } \\
\text { Hinterbliebenensicherung um ein Jahr }\end{array}$ & $\begin{array}{c}-0,09 \text { v.H. } \\
\text { (BM: }-3,5 \text { v.H.) }\end{array}$ \\
\hline $\begin{array}{l}\text { Nichterwerbstätigkeit als } \\
\text { Anspruchsvoraussetzung in der } \\
\text { Hinterbliebenensicherung }\end{array}$ & $-1,12$ v.H. \\
\hline $\begin{array}{l}\text { Erhöhung des Startalters in der } \\
\text { Hinterbliebenensicherung um ein Jahr }\end{array}$ & $\begin{array}{c}-0,07 \text { v.H. } \\
\text { (BM: }-2,6 \text { v.H.) }\end{array}$ \\
\hline Erhöhung der Wartezeit um ein Jahr & $\begin{array}{c}\text {-0,27 v.H. } \\
\text { (BM: }-6,48 \text { v.H.) }\end{array}$ \\
\hline $\begin{array}{l}\text { Erhöhung der Wartezeit um ein Jahr unter } \\
\text { Annahme einer Proratisierung der Ansprüche }\end{array}$ & $\begin{array}{c}-0,15 \text { v.H. } \\
(\mathrm{BM}:-3,6 \text { v.H.) }\end{array}$ \\
\hline $\begin{array}{l}\text { Erhöhung des Leistungsniveaus um ein } \\
\text { Prozentpunkt }\end{array}$ & 1,98 v.H. \\
\hline
\end{tabular}

Tabelle 8: Kostenelastizitäten einzelner Determinanten

Im Biedenkopf-Miegelschen Ansatz sind zudem der versicherte Personenkreis und das Startalter der Hinterbliebensicherung wichtige Stellgrößen.

Wird der Leistungsanspruch an die Erfüllung einer Wartezeit gebunden, reduziert eine einjährige Wartezeit die Kosten um 0,27 v.H. In der BiedenkopfMiegelschen Ausgestaltung, die eine Wartezeit von 25 Jahre vorsieht, verringern sich die Kosten folglich um 6,48 v.H. Falls eine Proratisierung der Ansprüche implementiert wird, reduziert sich dieser Effekt entsprechend.

Eine ähnliche Wirkung - wenn auch in abgeschwächter Form - zeigt eine Verschiebung des Alters in der Hinterbliebenensicherung, ab dem ein Sicherungsanspruch im Todesfall eines Ehepartners besteht. Eine Erhöhung des Startalters um ein Jahr verringert die Kosten einer Bürgerrente um 0,09 v.H. Der Biedenkopf-Miegelsche Vorschlag (Hinterbliebenenversorgung ab dem 55. Lebensjahr) führt somit zu Einsparungen in Höhe von 3,6 v.H.

Das System einer eingeführten Bürgerrente beinhaltet also generell Kostenvorteile. Allerdings ist dieses Ergebnis vorsichtig zu interpretieren, da die be- 
stehenden Rentensysteme die Zielsetzung einer Lebensstandardsicherung anstreben, während eine Bürgerrente lediglich eine finanzielle Grundabsicherung gewährt. Eine Lebensstandardsicherung kann in einem solchen Rentenmodell nur in einem Zusammenwirken mit ergänzender, privatwirtschaftlich organisierter Vorsorge erreicht werden. Ein entscheidender Parameter, der eine Vorteilhaftigkeit einer Bürgerrente begründen oder widerlegen kann, ist also das Gesamteinkommen der Rentner.

In dem nachfolgenden Kapitel wird daher das Gesamteinkommen eines Altersrentners in einem Rentensystem auf der Grundlage der Mirkodaten des SOEP erhoben. Hierzu werden die Angaben einzelner Befragungspersonen mit Hilfe eines Modells zur Kategorisierung beruflicher Tätigkeiten in standardisierte Erwerbsverläufe überführt, anhand derer individuelle Rentenkonten erstellt werden können. In einer Anwendung der wesentlichen Bestimmungen des SGB VI bzw. der grundlegenden Regelungen einer Bürgerrente lassen sich dann die Rentenansprüche einer Person unmittelbar aus den Rentenkonten ableiten. 


\section{Die Einkommen der Altersrentner}

Die Altersrente in der gesetzlichen Rentenversicherung wird maßgeblich durch den Verlauf der individuellen Erwerbsbiographie bestimmt. Dieser Zusammenhang ist als Grundsatz der Beitragsäquivalenz ${ }^{113}$ der Rentenleistung in der Rentenformel festgeschrieben. In einer Bürgerrente hingegen existiert eine derartige Verbindung nur partiell, da nur eine Komponente, nämlich das Einkommen aus privater Vorsorge, den Einwirkungen der Erwerbsbiographie ausgesetzt ist. Nur dann, wenn ein ausreichendes Erwerbseinkommen erzielt wird, kann für das Alter zusätzlich vorgesorgt werden.

Für einen Einkommensvergleich ist es also erforderlich, den Verlauf einer Erwerbsbiographie auszuwerten. Hierzu werden die Informationen zum beruflichen Werdegang eines Individuums in einem Alter vom 15. bis maximal zum 65. Lebensjahr erhoben und mit Hilfe eines Verfahrens zur Kategorisierung beruflicher Tätigkeiten in standardisierte Tätigkeitsprofile überführt. Die Ermittlung der Tätigkeitsprofile erfolgt exemplarisch für die 16. Welle des SOEP (Jahr 1999) mit rund 20400 Befragungspersonen.

Zur Systematisierung der Untersuchung werden aus der Menge der individuellen Profile Verlaufsmuster, d.h. Erwerbsbiographien, generiert, die für eine bestimmte Kohorte typisch sind.

Hierzu ist das Kapitel in zwei Teile gegliedert:

Im ersten Teil wird die Methodik zur Ermittlung typischer Erwerbsbiographien beschrieben. Schwerpunkt dieses Abschnittes ist die Darstellung des im Zuge einer Clusteranalyse verwendeten Verfahrens zur Messung der Ähnlichkeit beziehungsweise der Distanz zwischen zwei standardisierten Tätigkeitsprofilen.

In zweiten Teil wird ein Vergleich der Einkommen der Altersrentner in den beiden Rentensystemen durchgeführt. Hierbei erfolgt nach einer allgemeinen Darstellung des Modells zur Bestimmung der Einkommen der Altersrentner eine Präsentation der empirischen Ergebnisse.

113 Es ist klar, dass in den bestehenden Systemen eine Beitragsäquivalenz nur im Prinzip umgesetzt ist. Man denke hier z.B. an die Diskussion über die „versicherungsfremden Leistungen". 


\subsection{Die Identifikation repräsentativer Erwerbsbiographien}

\subsubsection{Ermittlung individueller Tätigkeitsprofile}

Ein Tätigkeitsprofil beschreibt eine Folge oder eine Sequenz von nominal skalierten Tätigkeiten $\lambda_{i, t} \in \Lambda$. Dabei ist $\lambda_{i, t}$ die Art der beruflichen Beschäftigung, die ein Individuum $i$ in einem Jahr $t$ überwiegend ausübt. Hieraus folgen individuelle Sequenzen mit bis zu 51 Elementen. Die Menge der möglichen Tätigkeiten $\Lambda$ enthält die folgenden Elemente:

\section{Menge der Tätigkeiten}

\begin{tabular}{cc}
\hline Tätigkeit $\lambda_{i, t}$ & Beschreibung \\
\hline 1 & Schule, Studium \\
\hline 2 & Ausbildung, Lehre \\
\hline 3 & Wehr- oder Zivildienst \\
\hline 4 & Vollzeitbeschäftigung \\
\hline 5 & Teilzeitbeschäftigung \\
\hline 6 & Arbeitslosigkeit \\
\hline 7 & $\begin{array}{c}\text { Hausfrau / -mann } \\
\text { (keine Berufstätigkeit) }\end{array}$ \\
\hline 8 & Ruhestand \\
\hline 9 & Schwangerschaft, Sonstige Tätigkeit \\
\hline 10 & Keine Information
\end{tabular}

Tabelle 9: Menge der Tätigkeiten

Die grobgefasste Kategorisierung der beruflichen Tätigkeiten einer Person $i$ ermöglicht es, ungeachtet der vielfältigen individuellen Besonderheiten standardisierte Tätigkeitsprofile zu erstellen.

Werden die $\lambda_{i, t}$ auf der Ordinate und die Zeit $t$ auf der Abszisse abgebildet, ergibt sich für eine Beispielperson das in Abbildung 6 dargestellte Profil. Diese Person besucht vom 15. bis zum 20. Lebensjahr eine Schule $\left(\lambda_{t}=1\right)$ und absol- 
viert anschließend bis zum 23. Lebensjahr eine Lehre oder eine Ausbildung $\left(\lambda_{1}=2\right)$. In der Zeit vom 24. bis zum 42. Lebensjahr übt die Person eine Vollzeitbeschäftigung $\left(\lambda_{t}=4\right)$ aus, die im Alter von 26 bis 28 Jahren durch eine Schwangerschaft oder eine sonstige Tätigkeit $\left(\lambda_{t}=9\right)$ und im Anschluss daran durch eine zweijährige Periode einer Hausfrauen- bzw. Hausmannstätigkeit $\left(\lambda_{t}=7\right)$ unterbrochen wird. Im 43. und 44. Lebensjahr ist die Person arbeitslos $\left(\lambda_{t}=6\right)$ und nimmt dann eine Teilzeitbeschäftigung $\left(\lambda_{t}=5\right)$ auf. Mit 60 Jahren tritt die Beispielperson in den Ruhestand $\left(\lambda_{t}=8\right)$ ein.

\section{Tätigkeitsprofil einer Beispielperson}

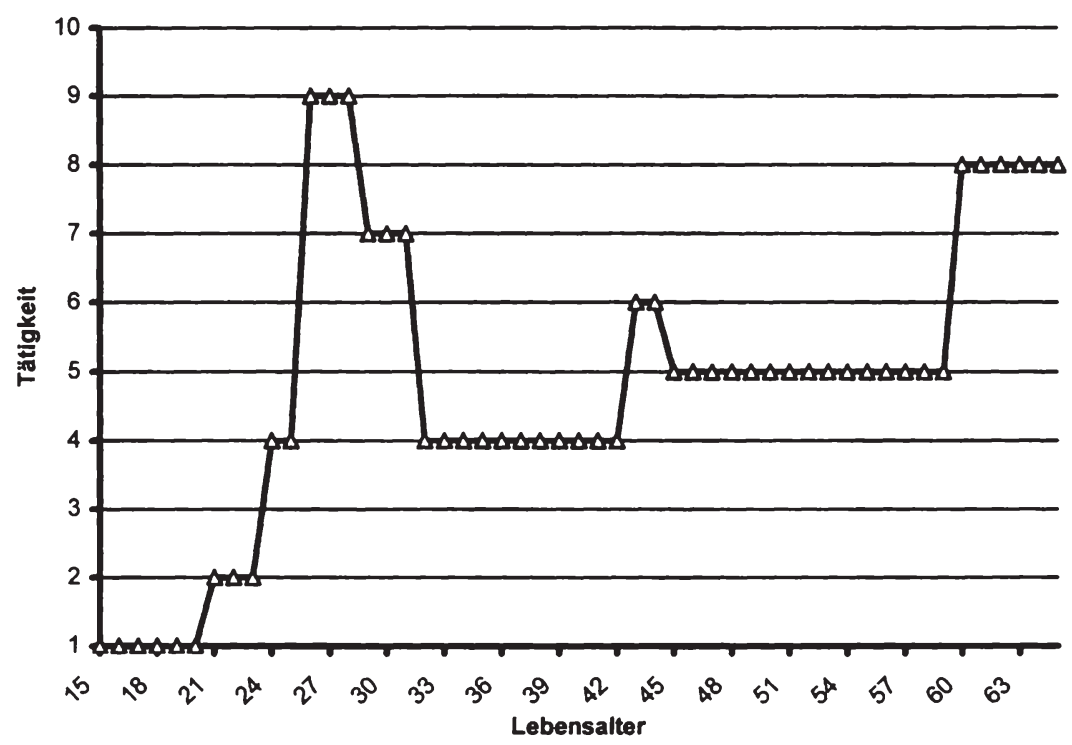

\section{Tätigkeiten}
(1) Schule, Studium
(2) Ausbildung, Lehre
(3) Wehr- / Zivildienst
(4) Vollzeitbeschäft.
(5) Teilzeitbeschäft.
(6) Arbeitslosigkeit
(7) Hausfrau /-mann
(8) Ruhestand
(9) Schwangerschaft, Sonstige Tätigkeit

(10) Keine Information

Abbildung 6: Tätigkeitsprofil einer Beispielperson ${ }^{14}$

114 Die Verbindungslinien bei Tätigkeitswechseln dienen ausschließlich der Illustration. 
Diese Vorgehensweise erlaubt es, die individuellen Erwerbsbiographien zu beschreiben und miteinander $\mathrm{zu}$ vergleichen. Zur Identifikation typischer Verlaufsmuster wird zunächst eine Clusteranalyse durchgeführt. Da es sich bei den Erwerbsbiographien um Folgen nominal skalierter Elemente handelt, wird die Ähnlichkeit zweier Sequenzen durch die „Optimal Matching Analysis“(OMA) bestimmt. Hierbei handelt es sich um ein Verfahren zur Sequenzmusteranalyse auf der Grundlage der Levenshtein-Distanz. ${ }^{115}$ Der folgende Abschnitt gibt einen Überblick über die wesentlichen Grundzüge dieses Distanzmaßes. ${ }^{116}$

\subsubsection{Optimal Matching Analysis ${ }^{117}$ - Eine Einführung}

Eine Sequenz ist eine Folge von Elementen eines endlichen Zustandsraumes $Z R .{ }^{118}$ Die Gesamtheit aller endlichen Sequenzen in $Z R$ sei mit $G S$ bezeichnet. Für eine endliche Sequenz es $\in G S$ mit $e s=\left(e s_{1}, \ldots, e s_{n}\right)$ gilt dann $e s_{1}, \ldots, e s_{n} \in Z R$.

Das Ziel besteht nun darin, die Ähnlichkeit zweier beliebiger Sequenzen im Sinne eines metrischen Distanzmaßes zu ermitteln. Hierzu wird eine Quellsequenz qs sukzessive durch Anwendung von elementaren Operationen $\omega \in \Omega$ in eine Zielsequenz zs überführt. Die Menge $\Omega$ der möglichen Operationen beinhaltet Einfügen, Löschen und Ersetzen einzelner Elemente einer Sequenz. Zudem sei angenommen, dass die Durchführung einer Operation $\omega$ mit Kosten in Höhe von $c o(\omega) \geq 0$ verbunden ist. Die Kosten werden nur von der Art der Operation (Einfügen, Löschen oder Ersetzen) bestimmt, nicht aber z.B. von der Position des Elementes in einer Sequenz. Hierbei sind $s b$ die Kosten für Er-

is Vgl. Levenshtein (1966). Ursprünglich wurde das Verfahren zur Ordnungsfindung innerhalb von Molekülketten in der Genforschung genutzt. Seit den Arbeiten von Abbott (1995), Abbott, DeViney (1992), Abbott, Hyrcak (1990) und Abbott, Forrest (1986)) ist dieses Verfahren auch in den Sozialwissenschaften zu finden. Hier wird OMA wie auch z.B. bei Erzberger, Prein (1997), Han, Moen (1999), Schaeper (1999), Aisenbrey (2000) oder Erzberger (2001) zur Bestimmung eines Distanzmaßes zwischen zwei Erwerbsprofilen verwendet. Für andere Anwendungen siehe z.B. Chan (1995) oder Halpin, Chan (1998).

116 Eine detaillierte Beschreibung findet sich bei Sankoff, Kruskal (1999).

117 Der folgende Abschnitt orientiert sich an Rohwer, Pötter (1999). Ausführlicher: Sankhof, Kruskal (1999), Abbott, Forrest (1990) und Abbott, Hrycak (1990).

118 Vgl. Abbott, Forrest (1990, S. 472). Mit Erzberger (2001, S. 136) kann eine Sequenz auch als „Abfolge von mindestens zwei aufeinanderfolgenden Übergängen in einer Prozesszeit verstanden" werden. 
setzen und id die Kosten für Einfügen oder Löschen. Das Ersetzen eines Elementes durch dasselbe Element bleibt kostenfrei.

Sei nun $q s\left[\omega_{1}, \omega_{2}, \ldots, \omega_{k}\right]$ die neue Sequenz, die sich nach der Anwendung der elementaren Operationen $\omega_{1}, \omega_{2}$ bis $\omega_{k}$ ergibt. Dann beschreibt der Ausdruck

$$
d_{\Omega}(q s, z s)=\min \left\{\sum_{i=1}^{k} c o\left(\omega_{i}\right) \mid z s=q s\left[\omega_{1}, \omega_{2}, \ldots, \omega_{k}\right], \omega_{i} \in \Omega, k \geq 0\right\}
$$

die kostenminimale Distanz zwischen den Sequenzen $q s$ und $z s$. Da diese Distanz streng positiv ist, nur dann den Wert Null annimmt, wenn Quell- und Zielsequenz übereinstimmen, und zudem die Dreiecksungleichung

$$
d_{\Omega}(q s, z s)+d_{\Omega}(z s, x s) \geq d_{\Omega}(q s, x s)
$$

erfüllt ist, handelt es sich um ein metrisches Distanzmaß.

Zur Ermittlung der kostenminimalen Distanz $d_{\Omega}(q s, z s)$ wird eine iterative Suchprozedur auf eine Distanzmatrix $D \in R^{n+1, n+1}$ angewendet. ${ }^{119}$ Innerhalb der Matrix erfolgt hierzu eine Bewegung von links oben nach rechts unten. Ein Schritt nach rechts bedeutet das Einfügen, ein Schritt nach unten steht für das Löschen, und ein Schritt nach rechts unten bedeutet das Ersetzen eines Elementes. Beschreibe $d_{p, q}: p, q=1, \ldots, n$ die minimalen Kosten, die anfallen, um in die Zelle in der $p$-ten Zeile und der $q$-ten Spalte zu gelangen und sei $d_{0,0}=0$. Die Distanzwerte in der nullten Zeile und Spalte der Matrix ergeben sich aus:

$$
\begin{aligned}
& d_{0, q}=d_{0, q-1}+i d \\
& d_{p, 0}=d_{p-1,0}+i d
\end{aligned}
$$

Sei die i-te Komponente einer Sequenz $q s$ mit $q s_{i}$ notiert, dann ergeben sich die Distanzwerte der übrigen Zellen in $D$ aus:

119 Hier seien nur Sequenzen mit einer identischen Anzahl von Elementen betrachtet. Sonst ergibt sich offenkundig eine Matrix $\operatorname{der}$ Form $D \in R^{n+1, m+1}$. 


$$
d_{p, q}=\min \left\{\begin{array}{l}
d_{p-1, q}+i d \\
d_{p-1, q-1}+\left\{\begin{array}{cc}
s b & \text { falls } q s_{p} \neq z s_{q} \\
0 & \text { sonst }
\end{array}\right. \\
d_{p, q-1}+i d
\end{array}\right.
$$

$d_{p, q}$ sind folglich die minimalen Kosten, die bei einer Überführung der Quellsequenz $q s$ zu einer Sequenz anfallen, die sich aus den ersten $p$ Elementen der Zielsequenz $z s$ und den letzten $n-q$ Elementen von $q s$ zusammensetzt. Die minimalen Kosten der kompletten Überführung einer Quell- in eine Zielsequenz sind $d_{n, n}$. Diese Kosten werden auch als Levenshtein-Distanz von Quell- und Zielsequenz bezeichnet.

Zur Verdeutlichung der oben beschriebenen Zusammenhänge wird die Levensthein-Distanz für ein einfaches Bespiel bestimmt, in dem zugleich die besondere Eignung der OMA herausgestellt werden kann. ${ }^{120}$

\subsubsection{Beispiel Distanzermittlung:}

Sei die Quellsequenz $(4,1,2,3)$ und die Zielsequenz $(1,2,3,4)$ gegeben. Die Kosten für elementare Operationen werden mit Schaeper (1999) auf 0,5 für Einsetzen und Löschen beziehungsweise auf 1,0 für Ersetzen festgelegt. ${ }^{121}$ Für die Distanzmatrix $D$ gilt Abbildung 7 .

Der Zellaufbau sei für den Fall der umrandeten Zelle erläutert: In der oberen linken Ecke werden die Substitutionskosten $s b$ notiert. Da im betrachteten Fall die Elemente der Quell- und der Zielsequenz übereinstimmen $q s_{2}=z s_{1}$, ist das Ersetzen kostenfrei. Die Kosten für Einfügen und Löschen sind in der oberen rechten Ecke beziehungsweise in der unteren linken Ecke eingetragen ${ }^{122}$;

120 Vgl. auch Abbildung 23, S. 169 für ein Beispiel zweier Tätigkeitsprofile.

121 Vgl. Schaeper (1999), S. 14. Denkbar ist auch, dass die Kosten bei einem Wechsel von einer Vollzeitberufstätigkeit zu einer Teilzeitbeschäftigung geringer sind, als bei einem Wechsel von einer Vollzeitbeschäftigung in die Arbeitslosigkeit. Vgl. hierzu z.B. Abbott, Forrest (1986), Halpin, Chan (1998) oder Chan (1995).

122 In der Literatur findet sich i.d.R. eine spiegelbildliche Notation der Kosten. Da ein Schritt nach rechts mit dem Einfügen und ein Schritt nach unten mit dem Löschen eines Elementes aus der Zielsequenz bzw. aus der Quellsequenz verbunden wird, ergibt hiermit sich eine intuitivere Zuordnung. Inhaltliche Unterschiede existieren nicht. Vgl. hierzu z.B. Abbot, Hrycak (1990), S. 178. 
annahmegemäß betragen diese stets $i d=0,5$. Der kostenminimale Distanzwert $d_{2,1}$ befindet sich in der unteren rechten Ecke und beträgt hier 0,5 Einheiten. Zusätzlich ist in jeder Zelle auch die Sequenz $q s\left[\omega_{1}, \omega_{2}, \ldots, \omega_{k}\right]$ angegeben, die den Verlauf nach Anwendung einer Folge von elementaren Operationen beschreibt, die die geringsten Kosten verursacht. Diese Sequenz besteht aus den ersten $p=1$ Elementen der Zielsequenz und den letzten $n-q=4-2$ Elementen der Quellsequenz, hier also die Zeichenfolge $(1,2,3)$.

Distanzermittlung mit OMA

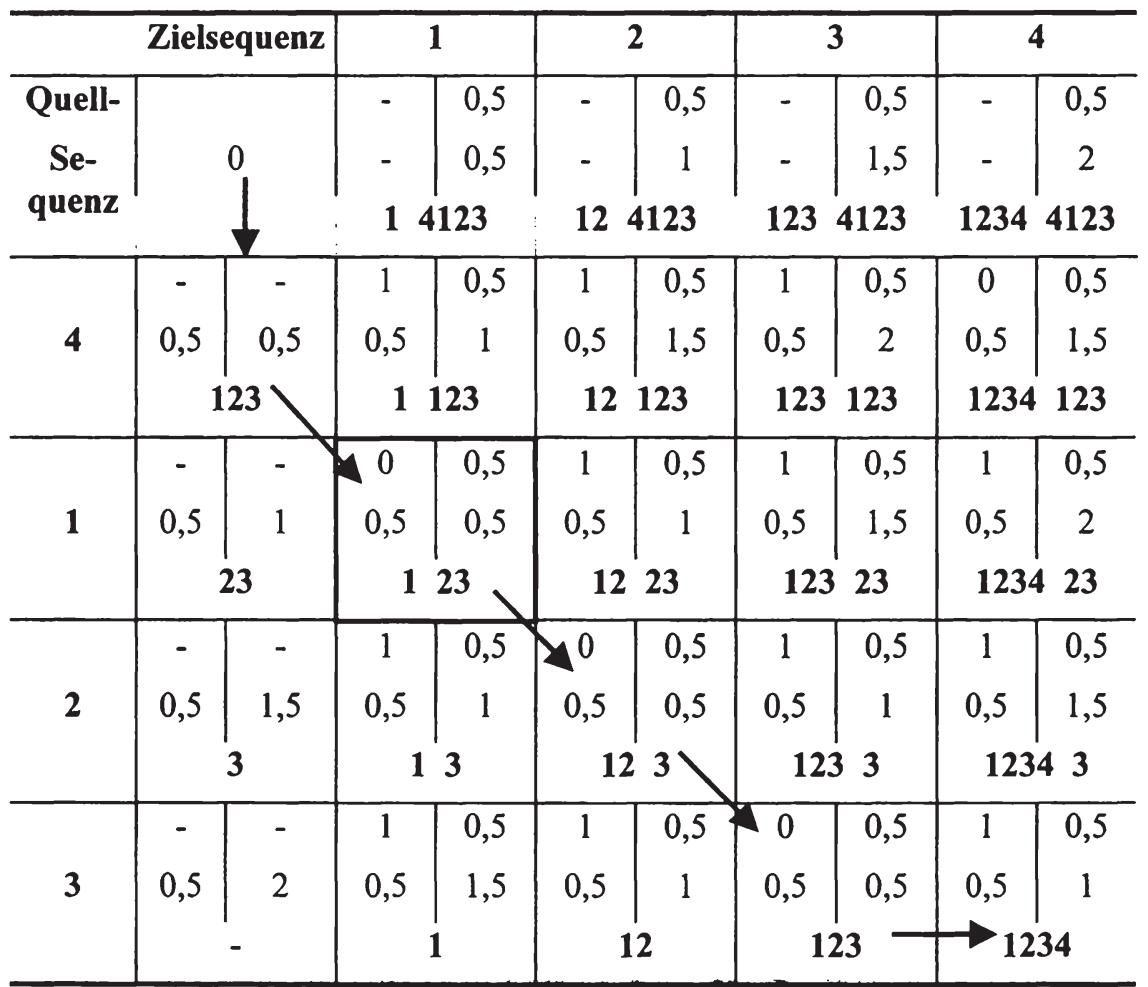

Abbildung 7: Distanzermittlung mit OMA 
Nach einer Division durch die Anzahl der Elemente in der Sequenz ergibt sich ein standardisiertes Distanzmaß $0<d_{n, n}<1$, die Levenshtein-Distanz. ${ }^{123}$ Stimmen Quell- und Zielsequenz überein, ergibt sich eine Distanz von Null, sind diese einander unähnlich, d.h. $q s_{i} \neq z s_{j} \forall i, j$, beträgt die Distanz eine Einheit. Im Beispiel ergibt sich also eine Distanz in Höhe von $\frac{d_{n, n}}{n}=0,25$.

Im Unterschied zu anderen Ähnlichkeits- oder Distanzmaßen nominal skalierter Variablen, die auf dem Prinzip des Paarvergleichs der Elemente in einer Sequenz basieren und somit eine vollkommene Unähnlichkeit ausweisen, ${ }^{124} \mathrm{er}-$ gibt sich bei Anwendung der „Optimal Matching Analysis“ eine geringe Distanz. Da die betrachteten Sequenzen nur um eine Periode gegeneinander verschoben sind, ist dies ein intuitiv leicht nachvollziehbares Ergebnis.

\subsubsection{Ermittlung typischer Verlaufsmuster}

\subsubsection{Gruppierung der individuellen Tätigkeitsprofile}

Zur Identifikation typischer Verlaufsmuster wird die Menge der individuellen Tätigkeitsprofile IT einer Geburtskohorte durch eine hierarchisch-agglomerative Clusteranalyse in disjunkte Cluster $I T_{j}: 1 \leq j \leq k$ zerlegt. ${ }^{125}$ Als Fusionsalgorithmus wird das Ward-Verfahren ${ }^{126}$ verwendet, und die Clustergröße folgt aus dem Elbow-Kriterium. Hierdurch werden die Profile beziehungsweise Gruppen von Profilen zu einer neuen Gruppe zusammengeschlossen, die die gruppeninterne Streuung am Wenigsten ansteigen lassen. Die optimale Clusteranzahl ergibt sich heuristisch, indem die Clusterzahl gegen die Streuungszuwächse in einem Diagramm abgetragen werden. Im Falle eines sprunghaften Anstiegs des Heterogenitätsmaßes ergibt sich ein Entscheidungshinweis. Am Beispiel der Geburtskohorte 1934 ergibt sich Abbildung 8.

123 Vgl. hierzu Abbott, Hrycak (1990), S. 154. Bei unterschiedlich langen Sequenzen wird durch die Anzahl der Elemente in der längeren Sequenz dividiert. Schaeper (1999, S. 14) und Windizio (2001, S. 183, Fußnote 13) teilen hingegen durch die Zahl der Elemente in der kürzeren Sequenz.

Vgl. z.B. der Tanimoto- oder Jaccard-, der Russel \& Rao- und der Simple Matching-Koeffizient. Siehe hierzu z.B. Backhaus (2003).

Es gilt somit $I T=\bigcup_{j=1}^{k} I T_{j}, I T_{p} \cap I T_{q}=\varnothing \forall p \neq q$.

Vgl. z.B. Aisenbrey (2000) oder Erzberger (2001). 


\section{Bestimmung der Clusterzahl mit dem „Ellbow-Kriterium“}

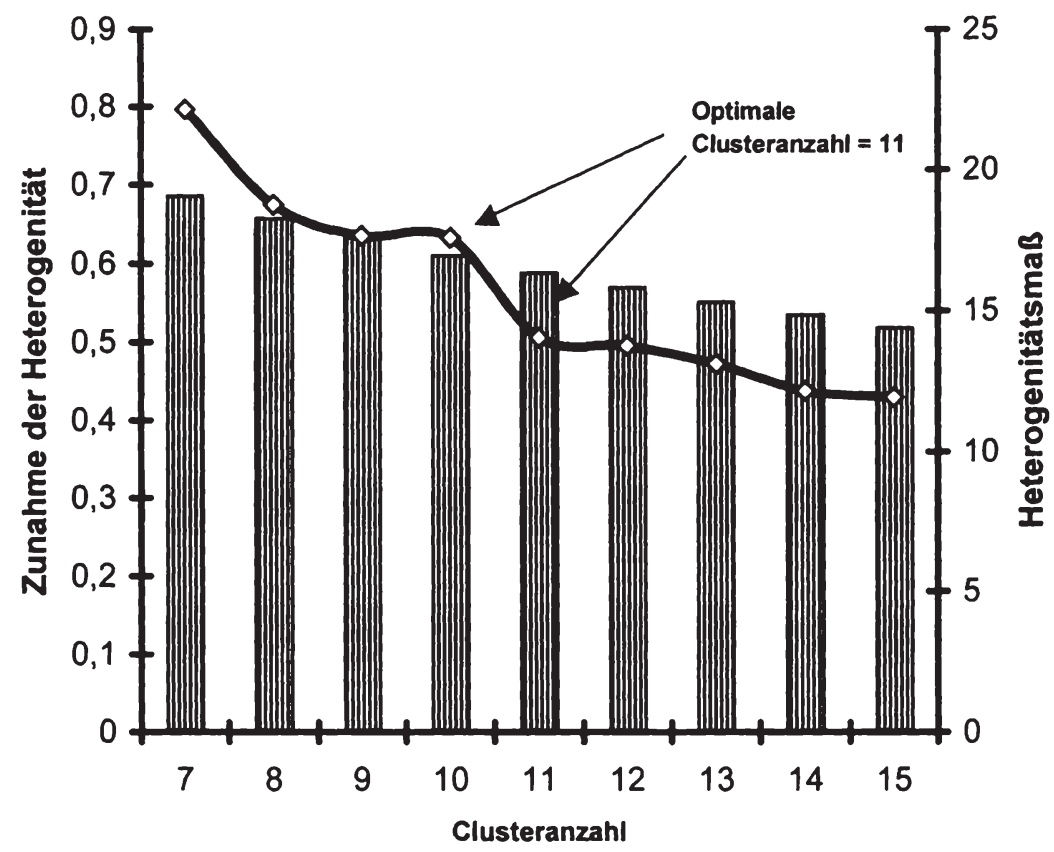

mmm Heterogenitätsmaß $\approx$ Zunahme des Heterogenitätsmaßes

Abbildung 8: Clusteranzahl nach dem „Ellbow“-Kriterium am Beispiel der Geburtskohorte 1934

In der Abbildung ist ein Ausschnitt aus dem Elbow-Diagramm zu sehen, in dem die absoluten Werte (gestreifte Säulen) und die Zuwächse (Liniendarstellung) des Heterogenitätsmaßes für verschiedene Clusteranzahlen abgebildet sind. Hier zeigt sich, dass die gruppeninterne Streuung besonders stark ansteigt, wenn zehn an Stelle von elf Gruppen gebildet werden.

Zur Beschreibung der Clusterungsergebnisse werden Maßzahlen bestimmt. Der Unterschiedsbetrag zwischen der durchschnittlichen Distanz $\overline{d_{\Omega}^{I T}}$ zweier be- 
liebiger Quell- und Zielsequenzen in der Menge individueller Tätigkeitsprofile $I T$ und der durchschnittlichen Distanz $\overline{d_{\Omega}^{I T_{j}}}$ zwischen zwei beliebigen Sequenzen innerhalb einer Gruppe $I T_{j}$ ist mit Abbot, Hrycak (1990) ${ }^{127}$ ein Gütemaß der Gruppierung. In Analogie hierzu beschreibt der Unterschied zwischen $\overline{d_{\Omega}^{I T_{j}}}$ und der durchschnittlichen Distanz $\overline{d_{\Omega}^{I T_{i} I T_{j}}}$ einer Sequenz in einer Gruppe $I T_{i}: 1<i<k$ zu einer beliebigen Sequenz in einer Gruppe $I T_{j}: 1<j<k, i \neq j$ die Trennschärfe zwischen diesen Gruppen.

\section{Güte der Gruppierung am Beispiel der Geburtskohorte 1934}

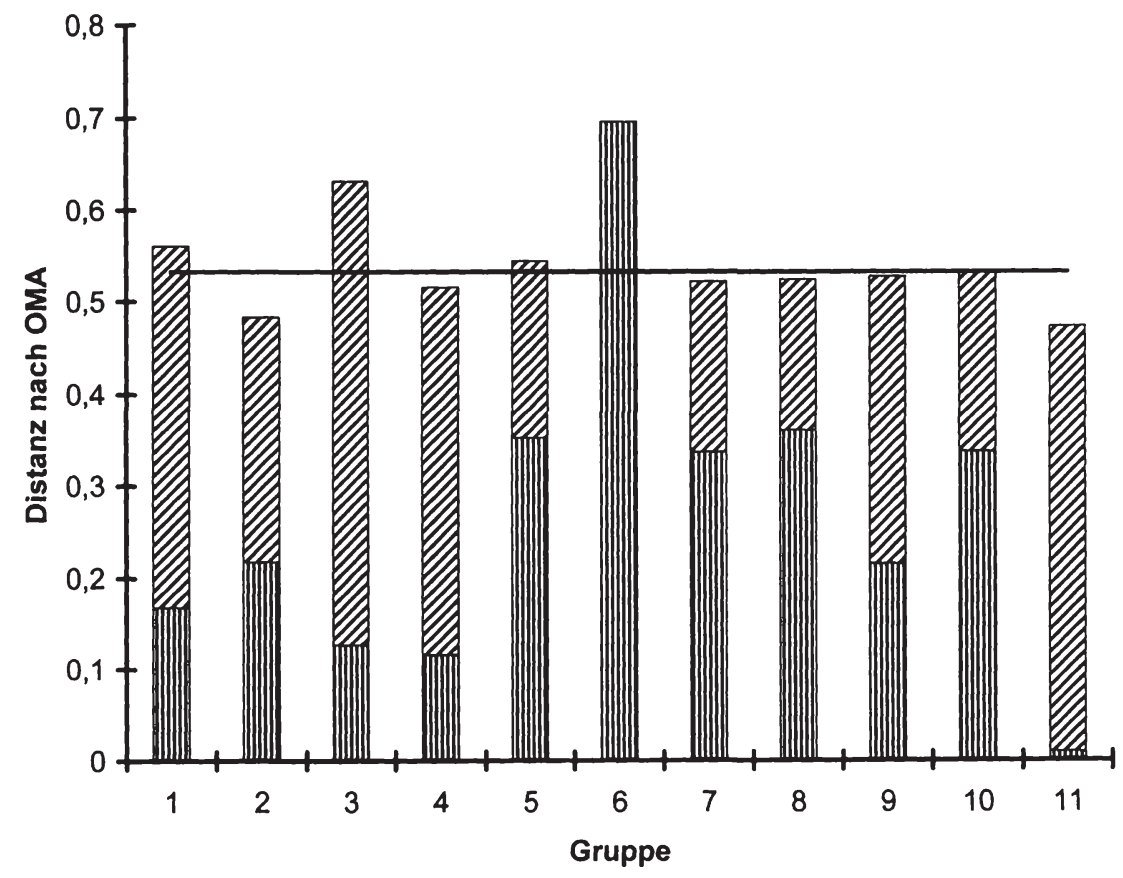

שa Distanz zu anderen Cluster m m Gruppeninterne Distanz —Distanz in der Grundgesamtheit Abbildung 9: Güte der Gruppierung am Beispiel der Kohorte 1934

127 Vgl. z.B. Abbot, Hrycak (1990), S. 165. 
Sofern $\overline{d_{\Omega}^{I T}}>\overline{d_{\Omega}^{I T_{j}}}$ und $\overline{d_{\Omega}^{I T_{j}}}<\overline{d m_{\Omega}^{I T_{i}, I T_{j}}}$ ergibt sich ein Hinweis auf eine sinnvolle Clusterung.

Abbildung 9 illustriert die Ergebnisse am Beispiel der Geburtskohorte 1934. In der Grafik sind die durchschnittlichen gruppeninternen Distanzen (vertikal gestreifte Balken) und die durchschnittlichen Distanzen einer beliebigen Sequenz in einer Gruppe zu einer Sequenz in einer anderen Gruppe (vertikal und quer gestreifte Balken) angegeben. Als Vergleichsmaßstab ist die durchschnittliche Distanz (Liniendarstellung) zweier beliebiger Sequenzen in der Menge der individuellen Tätigkeitsprofile eingezeichnet.

Am Beispiel der Geburtskohorte 1934 zeigt sich eine sinnvolle Clusterung. Während die durchschnittliche Distanz in der Menge der individuellen Tätigkeitsprofilen bei 0,53 Einheiten liegt, ergibt sich z.B. für die dritte Gruppe eine gruppeninterne Distanz in Höhe von rund 0,13 und eine durchschnittliche Dis$\operatorname{tanz} \mathrm{zu}$ Elementen in anderen Gruppen in Höhe von $0,63 .{ }^{128}$

\subsubsection{Reduktion auf einen typischen Verlauf}

Ausgehend von der in disjunkte Gruppen $I T_{j}$ zerlegten Menge individueller Tätigkeitsprofile werden typische Verlaufsmuster für eine Gruppe $I T_{j}$ bestimmt. Diese ergeben sich als Folge typischer Tätigkeiten $\lambda \in \Lambda$. Typisch sei die Tätigkeit $\lambda_{i, t}$, die von der überwiegenden Anzahl der Personen, die die Individuen $i$ in einer Gruppe $I T_{j}$ repräsentieren, zum Zeitpunkt $t$ ausgeübt wird.

Sei $G E_{i}$ das Gewicht eines Individuums $i$ in der Grundgesamtheit, d.h. der für eine repräsentative Betrachtung erforderliche Hochrechnungsfaktor und $I M_{I T_{j}}$ die Menge der Individuen in Gruppe $I T_{j}$. Die Wahrscheinlichkeit $P_{i, r}(\lambda)$, dass ein beliebiges Individuum $i$ zu einem Zeitpunkt $t$ eine bestimmte Tätigkeit $\lambda$ ausübt unter der Bedingung, dass $i$ zur Gruppe $I T_{j}$ zählt (d.h. $i \in I M_{I T_{j}}$ ), ergibt sich aus:

128 Eine Ausnahme bildet hier die sechste Gruppe, in der $\overline{d_{\Omega}^{I T}}>\overline{d_{\Omega}^{I T_{j}}}$ gilt. Begründet ist dies vermutlich in der geringen Anzahl der Elemente in dieser Gruppe. 
(9)

$$
P_{i, r}(\lambda)=\frac{\sum_{i: \lambda_{i, j}=\lambda} G E_{i}}{\sum_{i} G E_{i}}: i \in I M_{I T,}
$$

Hierbei beschreibt der Nenner die Summe der Gewichte der Personen in $I T_{j}$ der Zähler die Summe der Gewichte der Personen in Gruppe $I T_{j}$, die zum betrachteten Zeitpunkt die Tätigkeit $\lambda$ ausüben. Gleichung (9) beschreibt also den Quotienten aus der Anzahl der Personen in der Grundgesamtheit, die durch $I T_{j}$ repräsentiert werden und der Personenanzahl, die zur Gruppe $I T_{j}$ zählen und zum betrachteten Zeitpunkt Tätigkeit $\lambda$ ausüben. Insofern ist $P_{i, r}(\lambda)$ auch die Wahrscheinlichkeit, dass eine Person in der Bevölkerung der Bundesrepublik, die zur Gruppe $I T_{j}$ zählt, zum Zeitpunkt $t$ Tätigkeit $\lambda$ ausübt.

Die typische Tätigkeit zum Zeitpunkt $t$ ergibt sich dann aus dem Modalwert $P_{i, t}(\lambda)_{\bmod }$. Im Falle einer nicht eindeutigen Zuordnung wird die typische Tätigkeit der Vorperiode beibehalten bzw. im Sonderfall $t=15$, d.h. zu Beginn der Erwerbsbiographie, auf $\lambda=1$ (Schule, Studium) gesetzt.

Wird zur Illustration die Grundgesamtheit exemplarisch auf Personen der ersten Gruppe in der Geburtskohorte $1974^{129}$ beschränkt, ergeben sich die Wahrscheinlichkeiten $P_{i, t}(\lambda)$ aus Tabelle 10.

Die Wahrscheinlichkeit, dass eine Person, die zur ersten Gruppe der Geburtskohorte 1974 zählt, im Alter von z.B. 15 Jahren Tätigkeit $\lambda=1$ (Schule oder Hochschule) ausübt, beträgt 56 v.H. Die typischen Tätigkeiten für Personen in diesem Cluster zu einem Zeitpunkt $t=15, \ldots, 25$ sind umrahmt. Es resultiert der in der Tabelle 10 abgebildete Verlauf.

Im Anschluss an eine Schul- oder Studienzeit wird vom 20. bis zum 22. Lebensjahr eine Ausbildung oder eine Lehre absolviert. Danach üben die Individuen des ersten Clusters eine Vollzeitbeschäftigung aus. Bezüglich der Tätigkeiten im Alter von 25 Jahren liegen zum Zeitpunkt der Erhebung noch keine Daten vor. ${ }^{130}$

129 Diese Kohorte eignet sich aufgrund der geringen Anzahl der Elemente in einer Sequenz besonders für illustrative Zwecke.

130 Dies ist vermutlich auf den Zeitpunkt der Befragung zurückzuführen, die i.d.R. im ersten Quartal eines Jahres erfolgt. 


\section{Ermittlung der typischen Tätigkeiten am Beispiel des ersten} Clusters in der Geburtskohorte 1974

\begin{tabular}{|c|c|c|c|c|c|c|c|c|c|c|c|}
\hline & $t=15$ & $t=16$ & $t=17$ & $t=18$ & $t=19$ & $t=20$ & $t=21$ & $t=22$ & $t=23$ & $t=24$ & $t=25$ \\
\hline$P_{i, s}(\lambda=1)$ & 0,56 & 1,00 & 0,99 & 0,98 & 0,53 & 0,26 & 0,16 & 0,11 & 0,00 & 0,01 & 0,09 \\
\hline$P_{i, r}(\lambda=2)$ & 0,00 & 0,00 & 0,01 & 0,01 & 0,18 & 0,28 & 0,42 & 0,39 & 0,38 & 0,27 & 0,08 \\
\hline$P_{i, \lambda}(\lambda=3)$ & 0,00 & 0,00 & 0,00 & 0,00 & 0,06 & 0,23 & 0,20 & 0,09 & 0,02 & 0,00 & 0,00 \\
\hline$P_{i, \lambda}(\lambda=4)$ & 0,00 & 0,00 & 0,00 & 0,01 & 0,01 & 0,03 & 0,01 & 0,13 & 0,39 & 0,36 & 0,07 \\
\hline$P_{i, t}(\lambda=5)$ & 0,00 & 0,00 & 0,00 & 0,00 & 0,02 & 0,00 & 0,00 & 0,10 & 0,00 & 0,16 & 0,00 \\
\hline$P_{i, t}(\lambda=6)$ & 0,00 & 0,00 & 0,00 & 0,00 & 0,00 & 0,08 & 0,11 & 0,07 & 0,14 & 0,12 & 0,00 \\
\hline$P_{i, s}(\lambda=7)$ & 0,00 & 0,00 & 0,00 & 0,00 & 0,14 & 0,00 & 0,02 & 0,00 & 0,02 & 0,01 & 0,00 \\
\hline$P_{i, 1}(\lambda=8)$ & 0,00 & 0,00 & 0,00 & 0,00 & 0,00 & 0,00 & 0,04 & 0,04 & 0,04 & 0,00 & 0,00 \\
\hline$P_{i, \lambda}(\lambda=9)$ & 0,00 & 0,00 & 0,00 & 0,00 & 0,07 & 0,11 & 0,06 & 0,00 & 0,01 & 0,07 & 0,00 \\
\hline$P_{i, \lambda}(\lambda=10)$ & 0,44 & 0,00 & 0,00 & 0,00 & 0,00 & 0,00 & 0,00 & 0,06 & 0,00 & 0,00 & 0,77 \\
\hline
\end{tabular}

Tabelle 10: Ermittlung der typischen Tätigkeiten am Beispiel des ersten Clusters in der Geburtskohorte 1974

Anhand der Tabelle 10 wird auch klar, dass im Einzelfall marginale Unterschiede zwischen den Wahrscheinlichkeiten einzelner Tätigkeiten den Verlauf der typischen Erwerbsbiographie bestimmen. Während beispielsweise im Alter von 16 bis 18 Jahren eine beliebige Person, die in dieses Cluster fiele, die als typisch identifizierte Tätigkeit mit einer Wahrscheinlichkeit in Höhe von z.T. mehr als 98 v.H. ausübt, wird die typische Tätigkeit im Alter von 20 oder 23 Jahren mit einer Wahrscheinlichkeit von lediglich 28 v.H. bzw. 39 v.H. durchgeführt. Zudem ist die Differenz zur Tätigkeit, die mit der zweitgrößten Wahrscheinlichkeit ausgeübt wird, mit zwei Prozentpunkten beziehungsweise einem Prozentpunkt denkbar gering. 
Zusammenfassend ermöglicht dieses Vorgehen, die in disjunkte Gruppen zerlegte Menge individueller Tätigkeitsprofile auf wesentliche Verlaufsmuster, d.h. typische Erwerbsbiographien, für eine Gruppe zu konzentrieren. Da die Datenbasis nur jährliche Angaben beinhaltet, ist zu vermuten, dass Tätigkeiten, die gewöhnlich nur kurzfristig, d.h. unterjährig, ausgeübt werden, unterrepräsentiert werden. Ein Indiz hierfür ist z.B. die geringe Anzahl der Personen mit Wehr- und Zivildienstzeiten. Zur Untersuchung der Wirkungsweisen dieser Zeiten wird daher auf das Kapitel 4.2.2.4 verwiesen.

\subsubsection{Darstellung ausgewählter Ergebnisse}

Zur Illustration werden exemplarisch fünf typische Erwerbsbiographien der Geburtskohorte 1934 vorgestellt. Der Verlauf dieser Biographien ist in der Abbildung 10 dargestellt.

Das Cluster 1 enthält Biographien, die infolge einer kurzen Schul- und Ausbildungszeit (bis zum 19. Lebensjahr) und einer sich daran anschließenden langfristigen Vollzeitberufstätigkeit (20. bis 62. Lebensjahr) an den Tätigkeitsverlauf eines Arbeiters oder eines Angestellten erinnern. Mit 63 Jahren beginnt der Ruhestand. Die Personen in diesem Cluster sind überwiegend (86,8 v.H.) männlich und leben zusammen mit ihrem Ehegatten ( 71,1 v.H.) in einem Haushalt beziehungsweise unterhalten als Hinterbliebene (20,8 v.H.) einen eigenständigen Haushalt und haben durchschnittlich 1,3 Kinder.

Die erreichte Schulbildung (Hauptschulabschluss: 45,6 v.H.; Realschulabschluss: 46,7 v.H.) und der berufliche Bildungsabschluss (Lehre: 43,2 v.H.; Ausbildung: 10,5 v.H.; ohne Berufsabschluss: 36,8 v.H.) unterstreichen das Bild eines einfachen Arbeiters oder Angestellten. Insgesamt repräsentiert dieser Verlauf 18,8 v.H. des Geburtsjahrganges. Im Hinblick auf die Option einer Proratisierung der Grundrentenansprüche ist die durchschnittliche Aufenthaltsdauer der Zuwanderer von Interesse. Hierbei ist festzustellen, dass alle Personen in diesem Cluster die von Biedenkopf-Miegel (1997) genannte Schwelle in Höhe von 25 Jahren erfüllen. Die durchschnittliche Aufenthaltsdauer der Immigranten (11,5 v.H.) liegt hier bei rund 30 Jahren.

Der Verlauf der zweiten Gruppe zeigt eine Mischung aus beruflicher und Hausfrauentätigkeit. Nach einer kurzen Schul- und Ausbildungszeit wird bereits im Alter von 17 Jahren eine Vollzeitbeschäftigung eingegangen. Ab dem 27. Lebensjahr wird eine Hausfrauentätigkeit ausgeübt und im Alter von 63 Jahren beginnt der Ruhestand. 


\section{Ausgewählte typische Erwerbsbiographien der Geburtskohorte 1934}

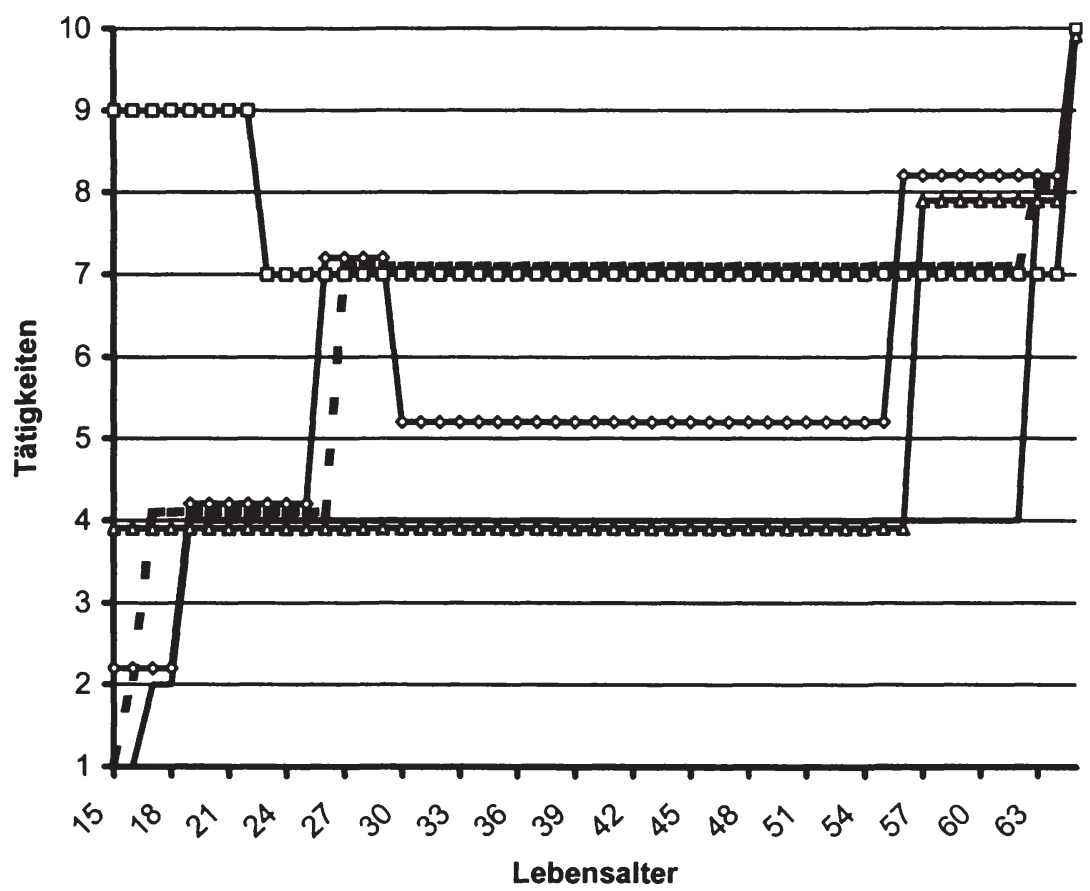

$\longrightarrow$ Cluster $1-$ Cluster $2 \longrightarrow$ Custer $3 \longrightarrow$ Cluster $4 \longrightarrow$ Cluster 7
(1) Schule, Studium
(2) Ausbildung, Lehre
(3) Wehr- / Zivildienst
(4) Vollzeitbeschäft.
(5) Teilzeitbeschäft.
(6) Arbeitslosigkeit
(7) Hausfrau / -mann
(8) Ruhestand
(9) Schwangerschaft, Sonstige Tätigkeit
(10) Keine Information

Abbildung 10: Ausgewählte typische Erwerbsbiographien der Geburtskohorte 1934

Die Gruppe besteht ausschließlich aus Frauen, die in einer Ehe (63,6 v.H.) einen gemeinschaftlichen Haushalt oder als Alleinstehende $(32,8$ v.H.) einen 
eigenständigen Haushalt unterhalten. ${ }^{131}$ Die durchschnittliche Kinderanzahl beträgt hier 0,8 Kinder pro Person. Im Bereich der Schul- und Berufsbildung zeigt sich hier ein gemischtes Bild. Zwar ist der Anteil der Personen mit allgemeiner Hochschulreife (10,7 v.H.) deutlich größer als im ersten Cluster (1,9 v.H.), jedoch verfügen immerhin 25,7 v.H. über keinen Schulabschluss. Entsprechend gering sind die beruflichen Qualifikationen (Lehre: 52,3 v.H.; ohne berufliche Bildungsabschluss: 28,6 v.H.). Die Gruppe bildet 21,0 v.H. des Jahrgangs $1934 \mathrm{ab}$. Hiervon leben 4,5 v.H. weniger als 25 Jahre in Deutschland. Die durchschnittliche Verweildauer der Immigranten liegt in dieser Gruppe bei 18,5 Jahren.

Die dritte Gruppe besteht zu gleichen Teilen aus beiden Geschlechtern und ist vom Verlaufsmuster mit der Biographie eines ungelernten Arbeiters in Verbindung zu bringen, weil vom 15. bis zum 56. Lebensjahr durchgängig eine Vollzeitbeschäftigung ausgeübt wird. Danach erfolgt der Renteneintritt. Die Gruppenmitglieder sind überwiegend (57,9 v.H.) verheiratet und haben durchschnittlich 1,1 Kinder. Die erreichte Schul- bzw. Berufsbildung betont das Bild eines ungelernten Arbeiters, da die Personen mehrheitlich über keinen Schulabschluss (61,2 v.H.) und keinen beruflichen Bildungsabschluss (60,3 v.H.) verfügen. Diese Gruppe repräsentiert die Erwerbsbiographie von 33,8 v.H. der Personen in der Geburtskohorte. Die durchschnittliche Aufenthaltsdauer der Zuwanderer beträgt hier 31,8 Jahre, d.h. es handelt sich überwiegend um langfristig in Deutschland lebenden Personen.

Mit nur 4,3 v.H. bildet die vierte Gruppe nur einen geringen Anteil der Geburtskohorte ab. Nach einer Schwangerschaft oder sonstigen Tätigkeit im Alter von 15 bis 22 Jahren wird eine Haufrauentätigkeit bis zum 64. Lebensjahr ausgeübt. Über die Tätigkeit im Alter von 65 Jahren liegen noch keine Daten vor. Die Gruppe besteht ausschließlich aus Frauen, die alle mit dem Ehepartner in einem gemeinschaftlichen Haushalt leben und durchschnittlich 0,3 Kinder erziehen. Die schulische und berufliche Qualifikationen ist unterdurchschnittlich (Hauptschulabschluss: 100 v.H.; Lehre: 26,4 v.H.; ohne berufliche Bildungsabschluss: 73,6 v.H.). Die Gruppenmitglieder sind zu 45,4 v.H. Zuwanderer, die sich seit durchschnittlich 30,3 Jahren in Deutschland aufhalten.

Abschließend wird das Cluster 7 vorgestellt. Bei dieser Gruppe handelt es sich um eine typische Ehefrauenbiographie. Nach einer Ausbildung oder Lehre wird im Alter von 19 Jahren eine Vollzeitbeschäftigung eingegangen. Von 26 bis 29

131 Hier ist zu vermuten, dass das Merkmal „ledig“ z.T. mit „nicht verheiratet“ gleichgesetzt worden ist. 
Jahren wird eine Hausfrautätigkeit ausgeübt. Im 30. Lebensjahr erfolgt der Wiedereinstieg ins Berufsleben durch die Aufnahme einer Teilzeitbeschäftigung, und im Alter von 56 Jahren treten die Personen in diesem Cluster in den Ruhestand ein. Das Cluster besteht ausschließlich aus Frauen, die durchschnittlich 0,3 Kinder gebären. Die erreichte schulische (52,3 v.H. Sonstiges; 32,4 v.H. Realschule) und berufliche Bildung (32,4 v.H. Ausbildung; 15,3 v.H. Studium) ist in diesem Cluster überdurchschnittlich gut. Zuwanderer gibt es hier nicht.

\subsubsection{Zusammenfassung}

Aus den Daten zum beruflichen Werdegang von 20400 Befragungspersonen des SOEP können mit Hilfe eines Modells zur Kategorisierung beruflicher Tätigkeiten individuelle Tätigkeitsprofile erstellt werden, die die Erwerbsbiographie eines Individuums in einem Alter von 15 bis maximal 65 Jahren beschreiben. Aus der resultierenden Menge individueller Tätigkeitsprofile werden im Zuge einer Anwendung der „Optimal Matching Analysis“ als Distanzmaß einer hierarchisch-agglomerativen Clusteranalyse Tätigkeitsverläufe ermittelt, die für die Individuen einer Geburtskohorte der Jahre 1934 bis 1974 als typisch zu bezeichnen sind. Typisch sind hierbei die Tätigkeiten, die zu einem beliebigen Zeitpunkt innerhalb einer Erwerbsbiographie von der überwiegenden Anzahl der Gruppenmitglieder ausgeübt wird.

Im zweiten Teil dieses Kapitels werden nun die Ruhestandeinkommen der Altersrentner am Beispiel der typischen Erwerbsverläufe analysiert. Vor dem Hintergrund des grundlegenden Prinzips einer Bürgerrente, die verstärkte Betonung der betrieblichen und insbesondere der eigenständigen Vorsorge, d.h. der privaten Ersparnis, ergibt sich die Möglichkeit, biographische Besonderheiten adäquat zu berücksichtigen. Beispielsweise können Zeiten einer Ausbildung oder Lehre, eines Wehr- oder Zivildienstes oder zum Beispiel einer Schwangerschaft zu den Zeitpunkten innerhalb eines Erwerbslebens empirisch begründet angenommen werden, zu denen diese gewöhnlich oder "typisch“ durchgeführt oder ausgeübt werden.

Im Vergleich zu dem in der Literatur oftmals verwendeten, in der Realität aber zunehmend seltener anzufindenden Konstrukt eines Standardrentners, der sich durch eine ununterbrochene Vollzeitbeschäftigung und eine 45-jährige Versicherungsdauer auszeichnet, ergeben sich offenkundig Vorteile. Anstatt die Analyse auf einen Typus zu beschränken, gelingt die Ausweitung auf eine An- 
zahl typischer Erwerbsverläufe. Die bestehende Vielfältigkeit der Biographien kann folglich empirisch fundiert abgebildet werden.

\subsection{Ein Einkommensvergleich - GRV vs. BR}

Nach Ermittlung typischer Erwerbsbiographien wird die finanzielle Situation der Altersrentner in den bestehenden Systemen, hier am Beispiel der gesetzlichen Rentenversicherung und in einer Bürgerrente untersucht.

Die Analyse orientiert sich am derzeit gültigen Rechtsstand der GRV und erfolgt in Anlehnung an die ab Januar 2004 zu erstellenden Rentenauskünfte bzw. Renteninformationen der Rentenversicherungsträger. ${ }^{132}$ Auf Grundlage der typischen Erwerbsbiographien wird mit Annahmen bezüglich der Einkommensverläufe in der Erwerbsphase und einem pauschalisierten Verfahren zur Bestimmung der persönlichen Entgeltpunkte für rentenrechtliche Anrechnungszeiten ${ }^{133}$ auf die Höhe der bisher erworbenen und auf die bei Renteneintritt zu erwartenden Rentenansprüche geschlossen.

\subsubsection{Die Altersrente in der GRV}

Der Monatsbetrag einer Rente $P E_{i, t}^{G R V}$ einer gesetzlich versicherten Person $i$ ergibt sich aus der Rentenformel ${ }^{134}$ :

$$
P E_{i, t}^{G R V}=R F_{i} \cdot Z F_{i} \cdot R W_{i} \cdot E P_{i}^{G E S}
$$

Dabei ist $R F_{i}$ der Rentenartfaktor, $Z F_{i}$ der Zugangsfaktor einer Person $i, R W_{t}$ der aktuelle Rentenwert in einem Jahr $t$ und $E P_{i}^{G E S}$ die Gesamtanzahl an persönlichen Entgeltpunkten. Die $E P_{i}^{G E S}$ ergeben sich aus der Erwerbsbio-

132 Ab dem 01. Januar 2004 werden den Pflichtversicherten in der GRV in einem Alter ab dem vollendetem 27. Lebensjahr jährliche Rentenauskünfte erteilt. Vgl. hierzu $\S 109$ (1-3) SGB VI. Wesentlicher Inhalt der Bescheide ist neben den Informationen zu den bereits erzielten Rentenpunkten auch eine Prognose über die mit Vollendung des 65. Lebensjahres zu erwartende Rente. Als Grundlage der Hochrechnung dient dabei der Versicherungsverlauf in einem fünfjährigen Zeitintervall unmittelbar vor dem Stichtag. Die in diesem Zeitabschnitt erzielten Rentenpunkte werden bis zum Ruhestandseintritt fortgeschrieben. Vgl. Langen (2001), S. Iff.

134 Vgl. § 64 SGB VI. 
graphie $E P_{i}^{B I O}=\sum_{a=0}^{A} E P_{i}^{(a)}(a \in\{0, \ldots, A\}$ ist das Alter in Lebensjahren $)$ und aus der Bewertung der Kindererziehungszeiten $E P_{i}^{K I N D}$. Da sich die Betrachtung auf eine Regelaltersrente ${ }^{135}$ konzentriert, ist der individuelle Rentenart- und der Zugangsfaktor stets gleich Eins. ${ }^{136}$

Ein Entgeltpunkt beschreibt den Anteil des individuellen Einkommens am Durchschnittsentgelt $Y_{t}$ aller sozialversicherungspflichtig Beschäftigten. Da die Einkommen nicht oder nicht in geeigneter Form aus dem SOEP gewonnen werden können, werden diese modelliert. ${ }^{137}$ Hierzu wird mit Annahmen bezüglich einer durchschnittlichen Entwicklung auf das Einkommen $Y_{k, K}^{(a)}$ einer typischen Person $k$ einer Kohorte $K$ im Alter von $a$ Lebensjahren geschlossen. ${ }^{138} \mathrm{Y}_{k, K}^{(a)}$ ergebe sich dabei aus $\mathrm{Y}_{1}$ (mit $t=K+a$ ), gewichtet mit einer Anpassungsfunktion $\kappa(a)$. Diese Funktion beschreibe den durchschnittlichen Anteil des Einkommens $Y_{t, i(a)}$ aller ganzjährig sozialversicherungspflichtig Beschäftigten $i$ in einem Lebensalter von $a$ Jahren am Durchschnittseinkommen aller ganzjährig sozialversicherungspflichtig Beschäftigten $Y_{t}$. Das Einkommen $Y_{k, K}^{(a)}$ ergibt sich aus:

$$
\mathrm{Y}_{k, K}^{(a)}=\mathrm{Y}_{K+a} \cdot \kappa(a)
$$

Die resultierenden Einkommen einer typischen Person $k$ eines Jahrganges $K$ im Alter von $a$ Jahren sind in der Abbildung 11 als Anteil am Durchschnittsentgelt $Y_{t}$ aller sozialversicherungspflichtig Beschäftigten in einem Jahr $t=K+a$ angegeben.

Eine typische Person im Alter von $a=23$ Jahren erzielt also ein Einkommen in Höhe von 82,10 v.H. des Durchschnittsentgeltes, im Alter von $a=44$ Jahren sind es 109,58 v.H. Zur Illustration ist die Abbildung in drei Abschnitte eingeteilt. Im ersten Abschnitt, d.h. bis zum vollendeten 30. Lebensjahr, steigt das Einkommen stark an und verhart dann, im zweiten Abschnitt, auf einem

\footnotetext{
135 Vgl. $\$ 35$ Nr.1 SGB VI.

136 Vgl. $\$ 67 \mathrm{Nr} .1$ und $\S 77$ (2) Nr.l.

137 Die Daten zur Einkommenshöhe sind bei rund 51 v.H. der Befragungspersonen auf volle 138 Hundert DM-Beträge und bei immerhin 15 v.H. auf volle Tausend DM-Beträge gerundet.

Vgl. § 70 (1) Satz 1.
} 
annähernd konstanten Niveau. Im dritten Abschnitt ergibt sich eine weitere Steigerung der Bezüge um rund fünf Prozentpunkte. Aus diesem Ansatz resultiert also ein plausibler, intuitiv leicht nachvollziehbarer Einkommenspfad.

\section{Durchschnittlicher Einkommensverlauf}

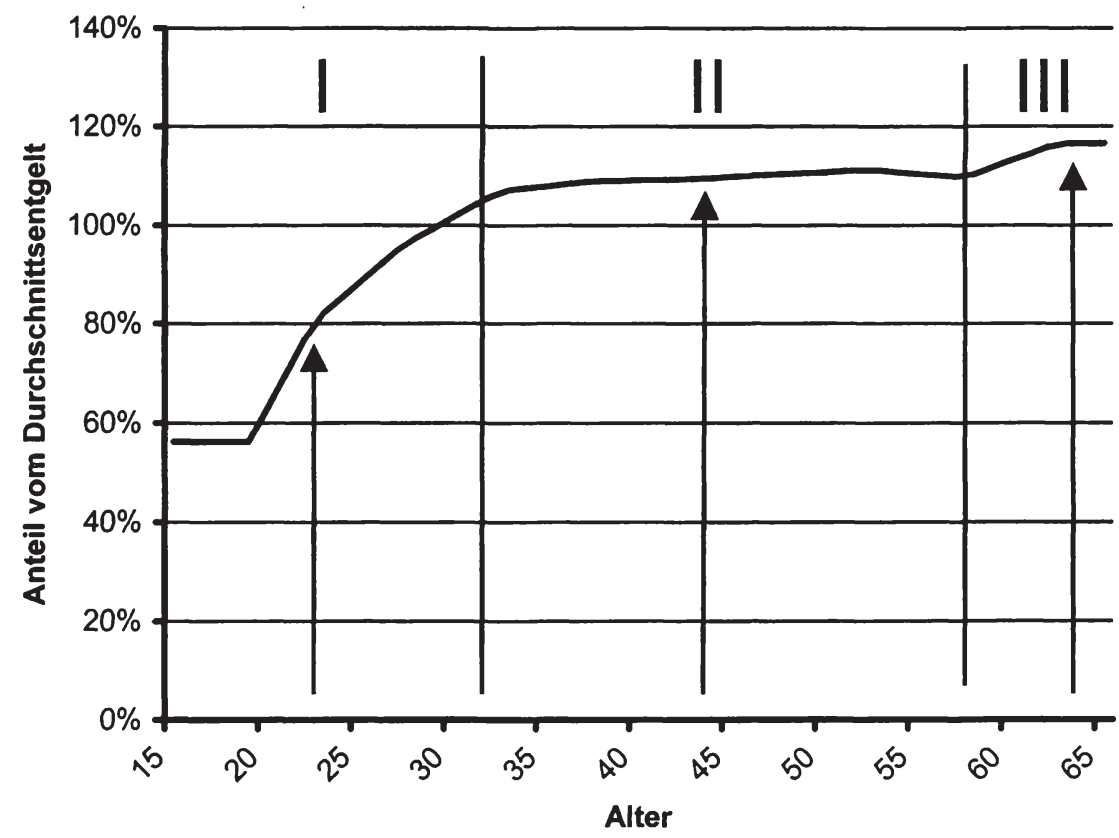

Abbildung 11: Durchschnittlicher Einkommensverlauf eines typischen Individuums ${ }^{139}$

Zur Bestimmung der Rentenansprüche aus einer Erwerbsbiographie werden die $\mathrm{Y}_{k, K}^{(a)}$ mit einem Tätigkeitsartfaktor $0 \leq \beta_{\lambda_{K+a}} \leq 1$ gewichtet, um die Abhängigkeit der Einkommen von der in einem Jahr $K+a$ ausgeübten Tätigkeit $\lambda_{K+a}$ auszudrücken. ${ }^{140}$

139 Quelle: Statistisches Bundesamt (lfd. Jahre E), S. 18f. Die angegebene Kurve ergibt sich nach einer Glättung der klassifizierten Daten. Im Alter von unter 19 Jahren ist die Kurve auf einem konstanten Niveau ergänzt worden.

140

Die Menge der möglichen Tätigkeiten ergibt sich aus Tabelle 9, S. 72. 
Wird bei einer Vollzeitbeschäftigung $\beta_{4}=1$ gesetzt, folgen beispielsweise im Alter von $a=23$ Jahren 0,8210 persönliche Entgeltpunkte beziehungsweise für $a=44$ Jahre 1,0958 Entgeltpunkte. Mit anderen Worten, die persönlichen Entgeltpunkte $E P_{k, K}^{(a)}$, die eine typische Person $k$ der Kohorte $K$ im Alter von $a$ Jahren bei einer Vollzeitbeschäftigung erzielt, ergeben sich unmittelbar aus der Abbildung 11. Wenn analog bei einer Teilzeitbeschäftigung $\beta_{5}=0,5$ angenommen wird, folgen die rentenrechtlichen Entgeltpunkte ebenso.

Im Falle einer Arbeitslosigkeit werden in Anlehnung an die gesetzlichen Bestimmungen 80 v.H. des jeweiligen Einkommens gewertet, ${ }^{141}$ d.h. es gilt $\beta_{6}=0,8$. Zur Ermittlung der Entgeltpunkte für Zeiten der beruflichen oder schulischen Ausbildung, des Wehr- oder Zivildienstes sowie für Zeiten der Schwanger- oder Mutterschaft wird ein pauschalisiertes Verfahren angewendet. Da die im SGB VI festgelegte Durchschnittsbewertung der Anrechnungszeiten $^{142}$ bei unvollständigen Biographien kaum sinnvoll sein kann, werden hier konstante, erwerbsverlaufunabhängige Werte angenommen.

Eine schulische Bildungsmaßnahme ab dem 17. Lebensjahr oder eine berufliche Maßnahme, hier die Tätigkeiten $\lambda=1$ oder $\lambda=2$, werden mit 0,75 Entgeltpunkten pro Versicherungsjahr berücksichtigt. Die Festlegung orientiert sich somit an den Maximalwerten für schulische oder berufliche Bildungsmaßnahmen. ${ }^{143}$

Für die Ableistung eines Wehr- oder Zivildienstes nach dem 01. Januar 2000 werden Einkommen in Höhe von 60 v.H. der Bezugsgröße gewertet. ${ }^{144}$ Im Vergleich zu den Regelungen früherer Jahre bedeutet dies offenkundig einen erheblichen Einschnitt. Da hier die Kohorten 1934 bis 1974 betrachtet werden, orientiert sich die Bewertung an einem Durchschnittswert in Höhe von 0,75 persönlichen Entgeltpunkten. ${ }^{145}$

\footnotetext{
141 Vgl. § $166(1)$ Nr. 2 SGB VI.

142 Vgl. § $71-\S 74$ SGB VI.

143 Vgl. $\$ 58(1)$ Nr. 4 und $\S 71$ i.V.m. $§ 74$ SGB VI.

144 Vgl. 18 (1) i.V.m. § 166 (1) Nr. 1 SGB VI.

145 Vgl. $\$ 256$ (3) SGB VI. Es wird also der Rechtsstand, der für die Ableistung von Wehrund Zivildienstzeiten vom 01. Januar 1982 bis zum 31. Dezember 1991 gültig war, zugrundegelegt. Sofern die tatsächliche Ableistung vor dem 31. Dezember 1981 erfolgte, ergibt sich eine geringfügige Unterschätzung der Rentenansprüche.
} 


\section{Berechnungsgrundsätze der GRV-Renten}

\begin{tabular}{|c|c|c|c|}
\hline $\begin{array}{c}\text { Tätigkeitsart } \\
\lambda_{t}\end{array}$ & Tätigkeit & $\begin{array}{c}\text { Entgeltpunkte oder } \\
\text { Tätigkeitsartfaktor } \\
\beta_{\lambda_{t}}\end{array}$ & $\begin{array}{c}\text { Rechtliche } \\
\text { Grundlage }^{146}\end{array}$ \\
\hline 1 & Schule, Studium & $\begin{array}{l}\text { Ab dem 17. Lebens- } \\
\text { jahr } 0,75 \mathrm{EP}\end{array}$ & $\begin{array}{c}\S 58(1) \text { Nr. } 4 \\
\text { und } \S 71 \text { i.V.m. } \\
\S 74 \text { SGB VI }\end{array}$ \\
\hline 2 & Ausbildung, Lehre & $0,75 \mathrm{EP}$ & $\begin{array}{c}\S 58(1) \text { Nr. } 4 \\
\text { und } \S 71 \text { i.V.m. } \\
\S 74 \text { SGB VI }\end{array}$ \\
\hline 3 & $\begin{array}{c}\text { Wehr- oder Zivil- } \\
\text { dienst }\end{array}$ & $0,75 \mathrm{EP}$ & $\begin{array}{l}\text { § } 256(3) \\
\text { SGB VI }\end{array}$ \\
\hline 4 & Vollzeitbeschäftigung & 1,0 & $\S 70$ (1) SGB VI \\
\hline 5 & Teilzeitbeschäftigung & 0,5 & $\S 70$ (1) SGB VI \\
\hline 6 & Arbeitslosigkeit & 0,8 & $\begin{array}{c}\S 166(1) \mathrm{Nr} .2 \\
\text { i.V.m. } \\
\S 70 \text { (1) SGB VI }\end{array}$ \\
\hline 7 & Hausfrau / -mann & 0,0 & $\begin{array}{c}\text { Folgt aus } \S 54 \\
\text { SGB VI }\end{array}$ \\
\hline 8 & Ruhestand & 0,0 & $\begin{array}{c}\text { Folgt aus } \S 54 \\
\text { SGB VI }\end{array}$ \\
\hline 9 & $\begin{array}{l}\text { Schwangerschaft } \\
\text { oder } \\
\text { Sonstige Tätigkeit }\end{array}$ & $0,0^{147}$ & $\begin{array}{c}\S 56(1) \\
\text { i.V.m. } § 249(1) \\
\text { SGB VI }\end{array}$ \\
\hline 10 & Keine Information & 0,0 & - \\
\hline
\end{tabular}

Tabelle 11: Berechnungsgrundsätze der GRV-Renten

Die Bewertung der Kindererziehung ist ein zentrales Leistungsmerkmal der gesetzlichen Rentenversicherung. Da die zur Bewertung erforderlichen Daten

\footnotetext{
146 Generell unberücksichtigt bleiben die Jährlichen Beitragsbemessungsgrenzen bzw. die Jährlichen Höchstwerte an Entgeltpunkten nach Anlage 2 bzw. 2b SGB VI.

147 Die Bewertung der Kindererziehungszeiten erfolgt separat.
} 
i.d.R. nicht aus dem Verlauf einer Erwerbsbiographie zu ermitteln sind, ${ }^{148}$ erfolgt eine pauschalisierte Einbeziehung. Die $E P_{k, K}^{K I N D}$ ergeben sich hierbei aus einer Bewertung der durchschnittlichen Kinderanzahl in einem Cluster mit jeweils einem Entgeltpunkt in den ersten drei Lebensjahren des Kindes. ${ }^{149}$ Der Tätigkeitsart $\lambda=9$ sind daher keine Entgeltpunkte oder Einkommen zugeordnet. ${ }^{150}$ Schließlich verbleiben die Tätigkeiten „Hausfrau oder Hausmann“, „Ruhestand“ sowie „Keine Information“ ohne Bewertung.

Ein Überblick über die Gesetzesquellen und Berechnungsgrundsätze, gegliedert nach Tätigkeitsarten, bietet die Tabelle 11. ${ }^{151}$

Auf Grundlage dieser Annahmen ist es möglich, die bisher erworbenen Rentenansprüche in der gesetzlichen Rentenversicherung zu bestimmen. Am Beispiel der typischen Person des ersten Clusters der Geburtskohorte 1934 sei die Bestimmung der Zahlbeträge der gesetzlichen Rentenversicherung in der Tabelle 12 exemplarisch dargestellt:

Die Person übt im Laufe des Erwerbslebens drei verschiedene Tätigkeiten aus. Im 15. und 16. Lebensjahr besucht die Person (vermutlich) eine Schule, so dass hierfür keine Entgeltpunkte gutgeschrieben werden. Während der sich anschließenden zweijährigen Ausbildungs- oder Lehrzeit werden insgesamt 1,50 persönliche Entgeltpunkte angerechnet. In der Vollzeitberufstätigkeit vom 19. bis zum 62. Lebensjahr erzielt die Person weitere 45,21 Punkte, so dass sich insgesamt 46,71 Entgeltpunkte aus der Erwerbsbiographie ergeben. Da die Personen in diesem Cluster durchschnittlich 1,31 Kinder erziehen, folgen insgesamt 50,64 persönliche Entgeltpunkte. In Größen des Jahres 1999 führt dies mit Gleichung (10) zu einer Bruttorente in Höhe von $€ 1248 .{ }^{152}$ Nach Abzug der Beiträge zur Kranken- und Pflegeversicherung der Rentner (KVdR und

148 Man denke hier z.B. an eine Familie mit nur einem Einkommen.

149 Diese Festlegung orientiert sich an dem Rechtsstand, der für Neugeborene ab dem 01. Januar 1992 maßgeblich ist. Hiernach werden in den ersten drei Lebensjahren des Kindes 0,9996 Entgeltpunkte pro Jahr gutgeschrieben. Vgl. § 56 (1) i.V.m. $§ 70$ (2) und $\S 249$ (1) SGB VI.

150 Vgl. $§ 57$ i.V.m. $§ 71$ (3), 72 (1), 73 SBG VI. Eine Begrenzung nach Anlage 2b SGB VI erfolgt nicht.

151 Vgl hierzu auch Tabelle 9, S. 72.

152 Bei einem Rentenwert in Höhe von $€$ 24,69. Quelle: VDR (2000), S. 240. 
PVdR) ergibt sich eine in beiden Systemen vergleichbare Nettozahlung in Höhe von $€ 1139$. $^{153}$

\section{Exemplarische Berechnung der GRV-Rentenansprüche}

\begin{tabular}{ccccc}
\hline Tätigkeit & Beginnalter & Endalter & $\begin{array}{c}\text { Dauer in } \\
\text { Jahren }\end{array}$ & $\begin{array}{c}\text { Entgelt- } \\
\text { punkte }\end{array}$ \\
\hline Schule, Studium & 15 & 16 & 2 & 0,00 \\
\hline Ausbildung, Lehre & 17 & 18 & 2 & 1,50 \\
\hline Vollzeitbeschäftigung & 19 & 62 & 44 & 45,21 \\
\hline Ruhestand & 63 & 64 & 2 & 0,00 \\
\hline Keine Information & 65 & 65 & 1 & 0,00 \\
\hline & & & Summe & 46,71 \\
\hline
\end{tabular}

Tabelle 12: Berechnung der persönlichen Entgeltpunkte

Der entsprechende Betrag in einer Grundrente ergibt sich aus den Zahlungen des obligatorischen Rentensystems zuzüglich der Einkommen aufgrund eigenständiger Vorsorge. Zur Quantifizierung dieser Zahlungen wird im folgenden Abschnitt die Sparfähigkeit einer typischen Person $k$ bestimmt.

\subsubsection{Die Einkommen der Altersrentner in einer Bürgerrente}

In einem System einer Bürgerrente ergeben sich die Einkommen der Altersrentner aus den obligatorischen Leistungen der Bürgerrente und den Einkommen, die aus privater Vorsorge resultieren.

Während die Bürgerrente vom Grundsatz her eine pro Kopf einheitliche Leistung bietet, die hier in Anlehnung an die Ausgestaltung anderer Grundrentensysteme proportional zur „Versicherungszeit“ angenommen wird, sind die Einkommen aus privater Vorsorge maßgeblich vom Verlauf der individuellen Erwerbsbiographie, d.h. der Fähigkeit zum Sparen, abhängig.

153 Die KVdR-/PVdR-Beiträge werden in Anlehnung an den Sachstand des Jahres 1999 in Höhe von 15 v.H. angenommen und im System der gesetzlichen Rentenversicherung nur zur Hälfte gewertet. Vgl. hierzu VDR(2000), S. 242. Von steuerlichen Aspekten wird abgesehen. 
Die Sparfähigkeit eines Individuums sei durch die Differenz der Beitragssätze $\tau_{t}^{R}$ in einem Rentensystem $R=\{B S, B R\}$ definiert. Sofern $\tau_{t}^{B S}>\tau_{t}^{B R}$ gilt, verfügen die Individuen in einer Bürgerrente über die Möglichkeit zur eigenständigen Vorsorge, ohne dass das verfügbare Einkommen in der Erwerbsphase geschmälert wird.

Im folgenden Abschnitt wird daher die Beitragsbelastung in einer Bürgerrente bestimmt, um mit Annahmen über die zu erzielenden Kapitalmarktrenditen auf die Höhe der Einkommen aus privater Vorsorge schließen zu können. Zur Einbeziehung der Auswirkungen unterschiedlicher Leistungshöhen einer Bürgerrente erfolgt die Untersuchung in dem bekannten Leistungsspektrum in Höhe von 50 v.H. bis 65 v.H. des Volkseinkommens pro Kopf. Zudem wird zwischen den beiden Hauptvarianten einer Bürgerrente, der Biedenkopf-Miegelschen Ausgestaltung (SEL/65/SEL65) und der weitergehenden Fassung (ALL/ 65/ALL18) differenziert.

\subsubsection{Die Beitragssätze in einem Grundrentensystem}

In Anlehnung an den institutionellen Rahmen der gesetzlichen Rentenversicherung ergibt sich der Beitragssatz in einem Grundrentensystem $\tau_{1}^{B R}$ aus:

$$
\tau_{t}^{B R} \cdot \mathrm{EM}_{t} \cdot \mathrm{Y}_{t}=\mathrm{TK}_{t}^{B R}-\mathrm{Z}_{t}^{B R}
$$

Hierbei beschreibt EM, die Anzahl der Erwerbstätigen, das sind die sozialversicherungspflichtig Beschäftigten, die Selbständigen, die Beamten und 80 v.H. der Arbeitslosen. Die Gesamtkosten eine Bürgerrente in einem Jahr $t$ werden mit $\mathrm{TK}_{t}^{B R}$ bezeichnet. Analog zur GRV sind die Kosten durch Beitragseinnahmen oder durch Zuschüsse des Bundes $Z_{t}^{B R}$ aus dem allgemeinen Steueraufkommen zu finanzieren. Die $Z_{t}^{B R}$ umfassen die Aufwendungen für die Versorgungsempfänger und die allgemeinen bzw. besonderen Zuschüsse an die gesetzliche Rentenversicherung. Hierbei werden Zuschüsse an die GRV proportional zur Reduktion der Gesamtkosten $\frac{\mathrm{TK}_{t}^{B R}}{\mathrm{TK}_{t}^{B S}}$ in einer Bürgerrente angepasst, um etwaige Mehrbelastungen in anderen Teilbereichen des Sozialsystems zu kompensieren. Man denke hier zum Beispiel an eine u.U. vermehrte Nachfrage nach BSHG-Leistungen in einer Biedenkopf-Miegelschen Ausgestaltung (SEL/65/SEL55). Somit beschreibt die linke Seite der Gleichung (12) 
die Beitragseinnahmen und die rechte Seite die durch Beitragszahlungen zu finanzierende Nettolast in einer Bürgerrente. Am Beispiel der beiden Hauptvarianten ALL/65/ALL18 ergeben sich die folgenden Beitragssätze:

\section{Beitragssätze in einer Bürgerrente (Variante ALL/65/ALL18)}

\begin{tabular}{c|c|c|c|c|c|c|c}
\hline & $\mathbf{1 9 9 5}$ & $\mathbf{1 9 9 6}$ & $\mathbf{1 9 9 7}$ & $\mathbf{1 9 9 8}$ & $\mathbf{1 9 9 9}$ & $\mathbf{2 0 0 0}$ & $\mathbf{2 0 0 1}$ \\
\hline $\mathbf{G R V}$ & $18,6 \%$ & $19,2 \%$ & $20,3 \%$ & $20,3 \%$ & $19,5 \%$ & $19,3 \%$ & $19,1 \%$ \\
\hline $\mathbf{5 0 , 0} \%$ & $10,5 \%$ & $10,5 \%$ & $10,6 \%$ & $10,2 \%$ & $10,0 \%$ & $10,2 \%$ & $10,3 \%$ \\
\hline $\mathbf{5 2 , 5} \%$ & $11,1 \%$ & $11,1 \%$ & $11,2 \%$ & $10,8 \%$ & $10,5 \%$ & $10,8 \%$ & $10,9 \%$ \\
\hline $\mathbf{5 5 , 0} \%$ & $11,7 \%$ & $11,7 \%$ & $11,8 \%$ & $11,4 \%$ & $11,1 \%$ & $11,4 \%$ & $11,5 \%$ \\
\hline $\mathbf{5 7 , 5} \%$ & $12,3 \%$ & $12,3 \%$ & $12,4 \%$ & $12,0 \%$ & $11,7 \%$ & $12,0 \%$ & $12,1 \%$ \\
\hline $\mathbf{6 0 , 0} \%$ & $12,9 \%$ & $12,9 \%$ & $13,0 \%$ & $12,7 \%$ & $12,3 \%$ & $12,6 \%$ & $12,7 \%$ \\
\hline $\mathbf{6 5 , 0} \%$ & $14,0 \%$ & $14,1 \%$ & $14,3 \%$ & $13,8 \%$ & $13,5 \%$ & $13,8 \%$ & $13,9 \%$ \\
\hline
\end{tabular}

Tabelle 13: Beitragssätze in der Bürgerrente am Beispiel der Variante ALL/65/ALL18

Im Jahr 1995 resultiert bei einem Leistungsniveau in Höhe von 50 v.H. des Volkseinkommens pro Kopf in der Variante ALL/65/ALL18 ein Beitragssatz in Höhe von 10,5 v.H. Bei Leistungen in Höhe von 65 v.H. sind es 14,0 v.H. des sozialversicherungspflichtigen Einkommens. Wird die Bürgerrente entsprechend der Biedenkopf-Miegelschen Anforderungen ausgestaltet, folgen geringere $\tau_{t}^{B R}$. Der Abschlag zur Variante SEL/65/SEL55 beträgt durchschnittlich 10,8 v.H. ${ }^{154}$ Kurzum, aufgrund der geringeren Kosten einer Bürgerrente verbleiben auch die Beiträge für alle hier betrachteten Leistungsniveaus beider Hauptvarianten unterhalb der Sätze der gesetzlichen Rentenversicherung, d.h. die Beitragssatzdifferenz ist stets positiv, $\tau_{t}^{G R V}-\tau_{t}^{B R}>0$.

Die Beitragssatzdifferenz ist ein Maß für die individuelle Sparfähigkeit. Infolge der reduzierten Beitragsbelastung verfügen die Individuen in den Jahren 1995 bis 2001 über zusätzliche Einkommen, welche zur eigenständigen Altersvorsorge eingesetzt werden können. Wird ein proportionales Verhältnis der $\tau_{t}^{B R} \mathrm{zu}$

154 Die Beitragssätze für die Variante SEL/65/SEL55 finden sich in Tabelle 22, S. 165. 
den Beiträgen in der GRV angenommen, gilt dies auch in allen anderen Jahren, d.h. die Individuen verfügen in der Erwerbsphase stets über eine Fähigkeit zur eigenständigen Vorsorge. Die Beiträge für ein bestimmtes Leistungsniveau einer Bürgerrente in einem beliebigen Jahr $t$ folgen dann aus:

$$
\tau_{t}^{B R}=\frac{\overline{\tau^{B R}}}{\tau_{t}^{G R V}}
$$

Hierbei beschreibt $\overline{\tau^{B R}}$ das arithmetische Mittel der Beiträge in den Jahren 1995 bis 2001.

Um Gewinner und Verlierer eines Systemwechsels festzustellen, wird nun untersucht, ob die Sparfähigkeit der Individuen ausreicht, um ein Gesamteinkommen in Höhe (bzw. oberhalb) der gesetzlichen Rente zu erzielen.

\subsubsection{Die vollständige Erwerbsbiographie}

Sei $P E_{k, K}^{R}$ der Monatsbetrag einer Rente an eine typischen Person $k$ der Kohorte $K$ in einem Rentensystem $R$ und $\tau^{K V}$ der Beitragssatz der Kranken- und Pflegeversicherung der Rentner.

Werden die Altersrenten der gesetzlich Versicherten als Maßstab zur Beschreibung der Ruhestandseinkommen in den bestehenden Alterssicherungssystemen verwendet, ergibt sich die durch eigenständige Vorsorge zu finanzierende Rentenlücke $P E_{k, K}^{G A P}$ für Personen, die sich bereits im Ruhestand befinden und daher über vollständige Erwerbsbiographien verfügen, aus:

$$
P E_{k, K}^{G A P}= \begin{cases}\left(1-\frac{1}{2} \tau^{K V}\right) \cdot P E_{k, K}^{G R V}-\left(1-\tau^{K V}\right) \cdot P E_{k, K}^{B R} & , \text { falls } P E_{k, K}^{G R V}>P E_{k, K}^{B R} \\ 0 & \text {, sonst }\end{cases}
$$

In Anlehnung an die Grundsätze des Altersvorsorge-Zertifizierungsgesetzes ${ }^{155}$ und einer durchschnittlichen weiteren Lebenserwartung der 65-Jährigen in Höhe von 23,5 Jahren für Frauen bzw. 15,6 Jahren für Männer hat der anzusparende Kapitalstock einen monatlichen Rentenbezug in Höhe von $P E_{k, K}^{G A P}$ bis

155 Vgl. $\$ 1(1)$ Nr. 5 AltZertG. 
zum 85 . Lebensjahr zu ermöglichen. ${ }^{156}$ Das hierzu notwendige Gesamtkapital $T C_{k, K}$ ergibt sich aus finanzmathematischen Grundsätzen.

Zur Bildung eines Kapitalstocks sei angenommen, dass die Individuen nur im Falle einer Vollzeitbeschäftigung eigenständig für das Alter vorsorgen können. Bei $\lambda \in\{1, . ., 3,5, \ldots, 10\}$ sei keine oder nur eine unzureichende Möglichkeit zum Sparen gegeben. Die mögliche Sparleistung $S_{k, K}^{(a)}$ einer typischen Person $k$ der Kohorte $K$ im Alter von $a$ Jahren ergibt sich aus:

$$
S_{k, K}^{(a)}=\left\{\begin{array}{lll}
Y_{k, K}^{(a)} \cdot\left(\tau_{t}^{G R V}-\tau_{t}^{B R}\right) & , \text { falls } \lambda_{k, K}^{(a)}=4 & \text { Sparleistung } \\
0 & , \text { sonst }
\end{array}\right.
$$

mit $t=K+a$

Hierbei sei $\lambda_{k, K}^{(a)} \in \Lambda$ die Tätigkeit, die eine typische Person $k$ im Alter von $a$ Lebensjahren ausübt. Werden nachschüssige Zahlungen angenommen, folgt die bis zu einem beliebigen Lebensjahr $a \in\left\{0, \ldots, a^{*}-1\right\}$ in der Erwerbsphase mögliche Gesamtsparleistung $T S_{k, K}^{(a)}$ aus:

$$
\begin{aligned}
& T S_{k, K}^{(0)}=S_{k, K}^{(0)} \\
& T S_{k, K}^{(1)}=\left(1+i_{K+1}^{s}\right) \cdot S_{k, K}^{(0)}+S_{k, K}^{(1)}
\end{aligned}
$$

(16) $T S_{k, K}^{(2)}=\left(1+i_{K+2}^{s}\right) \cdot\left[\left(1+i_{K+1}^{s}\right) \cdot S_{k, K}^{(0)}+S_{k, K}^{(1)}\right]+S_{k, K}^{(2)}$ Mögliches Sparkapital

$$
T S_{k, K}^{\left(a^{-}-1\right)}=\sum_{a=0}^{a^{*}-1}\left(S_{k, K}^{(a)} \cdot \prod_{j=a+1}^{a^{-}-1}\left(1+i_{K+j}^{s}\right)\right)
$$

In Analogie zur Systematik der Altersvorsorgeverträge kann $T C_{k, K}$ auch als durchschnittlicher Preis interpretiert werden, zu dem ein Versicherungsunternehmen - unter Ausschluss besonderer individueller Risiken - in der Lage ist, einer Person eine Leibrente in Höhe von $P E_{k, K}^{G A P}$ anzubieten. 
Hierbei bezeichnet $i_{K+a}^{s}$ den Żins in der Sparphase im Kalenderjahr $K+a .^{157}$

Das zusätzliche Einkommen, das aus $T S_{k, K}^{\left(a^{*}-1\right)}$ bis zum 85. Lebensjahr in konstanten Raten bezogen werden kann, wird mit $P E_{k, K}^{P R / V}$ bezeichnet.

Zur Identifikation von Gewinnern und Verlierern des fiktiven Systemwechsels wird folgendes Kennzifferkonzept eingeführt:

Wird (16) um einen Faktor $\mu_{k, K}>0 \quad \forall k, K$ in der Art erweitert, dass sich zum Zeitpunkt des Ruhestandseintritts ein Geldbetrag in Höhe des erforderlichen Gesamtkapitals $T C_{k, K}$ ergibt, d.h.

$$
T C_{k, K} \stackrel{!}{=} \sum_{a=0}^{a^{-}-1}\left(\mu_{k, K} \cdot S_{k, K}^{(a)} \cdot \prod_{j=a+1}^{a^{-}-1}\left(1+i_{K+j}^{s}\right)\right),
$$

dann folgt:

$$
T C_{k, K} \stackrel{!}{=} \mu_{k, K} \cdot T S_{k, K}^{\left(a^{\circ}-1\right)} .
$$

Der Faktor $\mu_{k, K}$ beschreibt also den Anteil an der möglichen Gesamtsparleistung $T S_{k, K}^{\left(a^{\cdot}-1\right)}$ eines typischen Individuums $k$ einer Kohorte $K$, der zum erforderlichen Gesamtkapital $T C_{k, K}$ führt. Falls $\mu_{k, K}<1$, ist das Individuum ein Gewinner in einer Bürgerrente, weil das Gesamteinkommen im Ruhestand der gesetzlichen Rente entspricht und das verfügbare Einkommen in der Erwerbsphase höher als in der GRV ist. Es handelt sich um einen Verlierer, falls $\mu_{k, K}>1$. In diesem Fall müssen in der Erwerbsphase zusätzliche Sparleistungen erbracht werden, die das verfügbare Nettoeinkommen schmälern.

157 Die Festlegung der Kapitalrendite ist offenkundig eine entscheidende Stellgröße in der Modellierung. Da die erzielbare Rendite neben der gesamtwirtschaftlichen Situation maßgeblich von der individuellen Risikopräferenz determiniert wird, orientiert sich die Festlegung an einem langfristigen Mittel. Vgl. hierzu: Das Deutsche Institut für Altersvorsorge (1998b, S. 50f). Unter der Berücksichtigung eines Risikoabschlages werden einheitlich 4,0 v.H. angenommen. Damit handelt es sich um eine zurückhaltende Schätzung der erzielbaren Renditen. Zur Rendite festverzinslicher Wertpapiere vgl. z.B. Sachverständigenrat zur Begutachtung der Gesamtwirtschaftlichen Entwicklung (1998), S. 384. 
Als eine zweite Kennziffer wird die Zeitdauer $D R_{k, K}$ in Jahren bestimmt, über die eine Person $k$ die Rentenlücke $P E_{k, K}^{G A P}$ aus eigenständiger Vorsoge $T S_{k, K}^{\left(a^{\circ}\right)}$ schließen kann. Hierbei ist $D R_{k, K}$ so definiert, dass der Mindestbezug bis zum 85. Lebensjahr den Nullpunkt markiert. Hierdurch ergibt sich eine leicht interpretierbare Größe, die Vorteile (Nachteile) einer Person $k$ in der Bürgerrente anzeigt, falls $D R_{k, K}>0\left(D R_{k, K}<0\right)$.

In einer weiteren Kennziffer wird ein direkter Einkommensvergleich ausgedrückt. Hierbei wird das Gesamteinkommen einer Person $k$ in einer Bürgerrente, hier also die Zahlungen des Rentesystems $P E_{k, K}^{B R}$ zuzüglich der Einkommen aus eigenständiger Vorsorge $P E_{k, K}^{P R I V}$, ins Verhältnis zur gesetzlichen Rente gesetzt. $P E_{k, K}^{P R I V}$ beschreibt dabei die maximal mögliche Rente, die bis zum 85 . Lebensjahr einer Person $k$ in konstanten Teilzahlungen aus $T S_{k, K}^{\left(a^{\circ}\right)}$ bezogen werden kann. $\pi_{k, K}$ ergibt sich aus:

$$
\pi_{k, K}=\left\{\begin{array}{ll}
\frac{P E_{k, K}^{B R}+P E_{k, K}^{P R V}}{P E_{k, K}^{G R V}} & , \text { falls } P E_{k, K}^{G R V}>0 \\
0 & , \text { sonst }
\end{array}\right. \text { Einkommensquotient }
$$

Der Einkommensquotient $\pi_{k, K}>1\left(\pi_{k, K}<1\right)$ beschreibt einen Gewinner (Verlierer).

\subsubsection{Die unvollständige Erwerbsbiographie}

Der Vorabschnitt untersucht die finanzielle Situation eines Altersrentners mit einer abgeschlossenen Erwerbsbiographie. Damit die Analyse auch auf jüngere Personen ausgeweitet werden kann, wird das Modell angepasst. In Anlehnung an die Renteninformationen ${ }^{158}$ der Rentenversicherungsträger wird bei Personen mit unvollständigen Erwerbsbiographien ein zweifacher Vergleich der finanziellen Vorteilhaftigkeit durchgeführt.

Zunächst erfolgt der Einkommensvergleich ausschließlich auf der Grundlage der bereits gesicherten Daten aus der Teilbiographie. In einem zweiten Ansatz

158 Vgl. § 109 i.d.F. ab 01.01.2004. In einer Ausweitung der Bestimmungen werden hier auch Personen in einem Alter von 25 Jahren einbezogen. 
wird dann mit Annahmen bezüglich der zukünftigen Entwicklung der Erwerbstätigkeit einer Person eine Prognose über die zum Zeitpunkt des Ruhestandeintritts zu erwartende Rente in beiden Systemen abgegeben. Insofern folgt dieser Ansatz unmittelbar dem methodischen Konzept der Renteninformationen der Rentenversicherungsträger. Die Festlegung des Verrentungsalters der Individuen orientiert sich dabei am derzeit gültigen Sachstand des SGB VI.

\section{Einkommen der Altersrentner zum Zeitpunkt der Erhebung}

Die bislang erzielten GRV-Rentenansprüche ergeben sich aus der Teilerwerbsbiographie und aus der Bewertung der Kindererziehungszeiten. Das Lebensalter zum Zeitpunkt der Erhebung sei mit $a^{\prime}$ bezeichnet. Die persönlichen Entgeltpunkte aus der Teilbiographie $E P_{k, K}^{B I O}$, d.h. die innerhalb des Intervalls $\left[0, a^{\prime}\right]$ erzielten Rentenpunkte, ergeben sich in Analogie zum Abschnitt 4.2.1. ${ }^{159}$ Zusammen mit den bis zum Alter von $a^{\prime}$ Jahren erreichten $E P_{k, K}^{K I N D}$ ergeben sich die $E P_{k, K}^{G E S}$. Die resultierende GRV-Rente folgt aus Gleichung (10).

In der Bürgerrente ist es erforderlich, einen anteiligen Grundrentenanspruch zu modellieren, um zu einer adäquaten Vergleichsgröße zu gelangen. In Anlehnung an die Ausgestaltung bestehender Grundrentensysteme (z.B. das kanadische OAS) wird eine Proratisierung der Rentenansprüche angenommen. Hierzu wird ein Proportionalitätsfaktor $\frac{1}{50} \leq \varepsilon_{k, K} \leq 1$ eingeführt, der den Bruttobetrag einer Bürgerrente proportional zur Dauer der bislang bekannten Erwerbsbiographie einer Person $k$ anpasst. Die durch eigenständige Vorsorge

$$
\begin{aligned}
& E P_{k, K}^{b i o}=\sum_{a=0}^{a^{\prime}-1} E P_{k, K}^{(a)} \text { mit } \\
& E P_{k, K}^{(a)}=\left\{\begin{array}{l}
0,75 \quad, \text { falls }\left(\lambda_{k, K}^{(a)}=1: a \geq 17\right) \text { und } \lambda_{k, K}^{(a)}=\{2,3\} \\
\beta_{\lambda_{1}} \cdot \frac{Y_{t}^{(a)}}{Y_{t}}, \text { falls } \quad \lambda_{k, K}^{(a)}=\{4,5,6\} \\
0 \quad, \text { sonst }
\end{array}\right. \\
& \text { und } t=K+a .
\end{aligned}
$$


zu finanzierende Rentenlücke $P E_{k, K}^{G A P}$ ergibt sich für Personen mit unvollständigen Erwerbsbiographien aus:

$$
P E_{k, K}^{G A P}= \begin{cases}\left(1-\frac{1}{2} \tau^{K V}\right) \cdot P E_{k, K}^{G R V}-\left(1-\tau^{K V}\right) \cdot \varepsilon_{k, K} \cdot P E_{k, K}^{B R} & , \text { falls } P E_{k, K}^{G R V}>\varepsilon_{k, K} \cdot P E_{k, K}^{B R} \\ 0 & \text {, sonst }\end{cases}
$$

Wird eine Mindestbezugsdauer in Höhe von 20 Jahren angenommen, ergeben sich das notwendige Gesamtkapital $T C_{k, K}$, das mögliche Sparkapital $T S_{k, K}^{\left(a^{\prime}-1\right)}$ und das mögliche Einkommen aus privater Vorsorge $P E_{k, K}^{P R I V}$ in Analogie zum Abschnitt 4.2.2.2.

Die Vergleichsgröße zum Zeitpunkt der Erhebung in einer Grundrente ist also

$$
\left(1-\tau^{K V}\right) \cdot \varepsilon_{k, K} \cdot P E_{k, K}^{B R}+P E_{k, K}^{P R V} \text {. }
$$

\section{Einkommen der Altersrentner zum Zeitpunkt des Renteneintritts}

In einem zweiten Ansatz erfolgt ein Vergleich zum Zeitpunkt des Renteneintritts. Hierfür ist es erforderlich, Annahmen bezüglich der Art der Erwerbstätigkeit und der Höhe der Einkommen in dem verbleibendem Zeitraum bis zum Renteneintritt zu treffen. Der Methodik der Renteninformationen der Rentenversicherungsträger folgend werden die Jahre $K+a^{\prime}$ bis $K+a^{*}$ mit Entgeltpunkten in Höhe eines Durchschnittswertes aus den letzten fünf Jahren der Erwerbstätigkeit bewertet. ${ }^{160}$ Implizit wird damit angenommen, dass eine Person $k$ bis zum Eintritt in den Ruhestand in jedem Jahr ein Einkommen in Höhe von

$$
\mathrm{Y}_{k, K}^{(a)}=\mathrm{Y}_{t} \cdot E P_{k, K}^{P R O G}: t=K+a, a^{\prime} \leq a<a^{*}
$$

erzielt.

Hierbei sei vereinbart, dass die Einkommen generell aus einer Vollzeitbeschäftigung resultieren, ${ }^{161}$ so dass eine Fähigkeit zum Sparen gegeben ist.

\footnotetext{
160 Im Unterschied zu den Renteninformationen wird eine Hochrechnung auch dann durchgeführt, wenn die Person in dem relevanten Fünfjahreszeitraum ausschließlich Beitragsgeminderte Zeiten aufweisen kann. Infolge der pauschalen Einbeziehung der Anrechungszeiten erscheint diese Verallgemeinerung möglich.

Es sei betont, dass dies eine starke Annahme ist.
} 
Die Wirkungsweise der Fortschreibungsregeln sei an einem Beispiel illustriert: Hierzu wird eine Beispielperson betrachtet, die vom 15. bis zum 65. Lebensjahr eine Vollzeitbeschäftigung ausübt. Die $E P_{k . K}^{(a)}$ folgen dann unmittelbar aus der Abbildung 11. Die Anpassung der hochgerechneten an die tatsächlich erreichten Entgeltpunkte kann dann für Erhebungen zu einem beliebigen Lebensalter $a^{\prime}$ durch das Verhältnis

$$
q^{\left(a^{\prime}\right)}=\frac{\left(a^{*}-1-a^{\prime}\right) \cdot E P^{P R O G}+\sum_{a=0}^{a^{\prime}-1} E P^{(a)}}{\sum_{a=0}^{a^{\prime}-1} E P^{(a)}}
$$

ausgedrückt werden. Aufgrund der stilisierten Erwerbsbiographie der Beispielperson, hier insbesondere die Annahme einer ununterbrochenen Ausübung einer Vollzeitbeschäftigung, nähert sich $q^{\left(a^{\prime}\right)}$ rasch dem Wert Eins. Bereits ab dem 31. Lebensjahr, d.h. mit Beginn des zweiten Abschnittes in der Abbildung 11 , beträgt die Anpassung über 90 v.H.

Anders verhält es sich, wenn in der Zeit bis zum Renteneintritt Tätigkeitswechsel zu berücksichtigen sind. Man denke hier beispielsweise an eine bis zum Alter von $a^{\prime}$ durchgängig vollzeitbeschäftigte Person, die in den Folgejahren die Erwerbstätigkeit (freiwillig oder unfreiwillig) einschränkt oder einstellt. Derartige Veränderungen können innerhalb der Prognose nicht abgebildet werden. Zur Illustration seien reale Erwerbsbiographien betrachtet. Hierzu werden für die Personen der Geburtskohorte 1934 zu verschiedenen Zeitpunkten Hochrechnungen über zum Zeitpunkt des Renteneintritts zu erwartenden Entgeltpunkte durchgeführt. Ist der tatsächliche Renteneintritt bekannt, ergibt sich die durchschnittliche Anpassung aus Abbildung 12.

Der Anpassungsverlauf ist stark geschlechtsspezifisch. Während sich für Männer im Falle einer Hochrechnung zum Zeitpunkt $a^{\prime}=35$ eine Anpassung von 99,6 v.H. ergibt, beläuft sich der entsprechende Wert für Frauen auf 122,9 v.H. Die Entgeltpunkte der Frauen werden also i.d.R. überschätzt. Vor dem Hintergrund eines oftmals anzutreffenden vorzeitigen Rückzugs der Frauen aus dem Erwerbsleben, z.B. durch einen Wechsel in eine Teilzeitbeschäftigung oder eine Hausfrauentätigkeit, ist dieses Ergebnis intuitiv leicht nachvollziehbar. Aufgrund der gleichförmigeren, weil ununterbrochenen Erwerbsbiographien sind die Abweichungen bei Männern deutlich geringer. Die angewandten Fort- 
schreibungsregeln können also durchaus Abweichungen beinhalten, die insbesondere bei sehr frühen Prognosezeitpunkten bedeutsam sind.

\section{Anpassung der Prognosewerte an die tatsächlichen Entgeltpunkte}

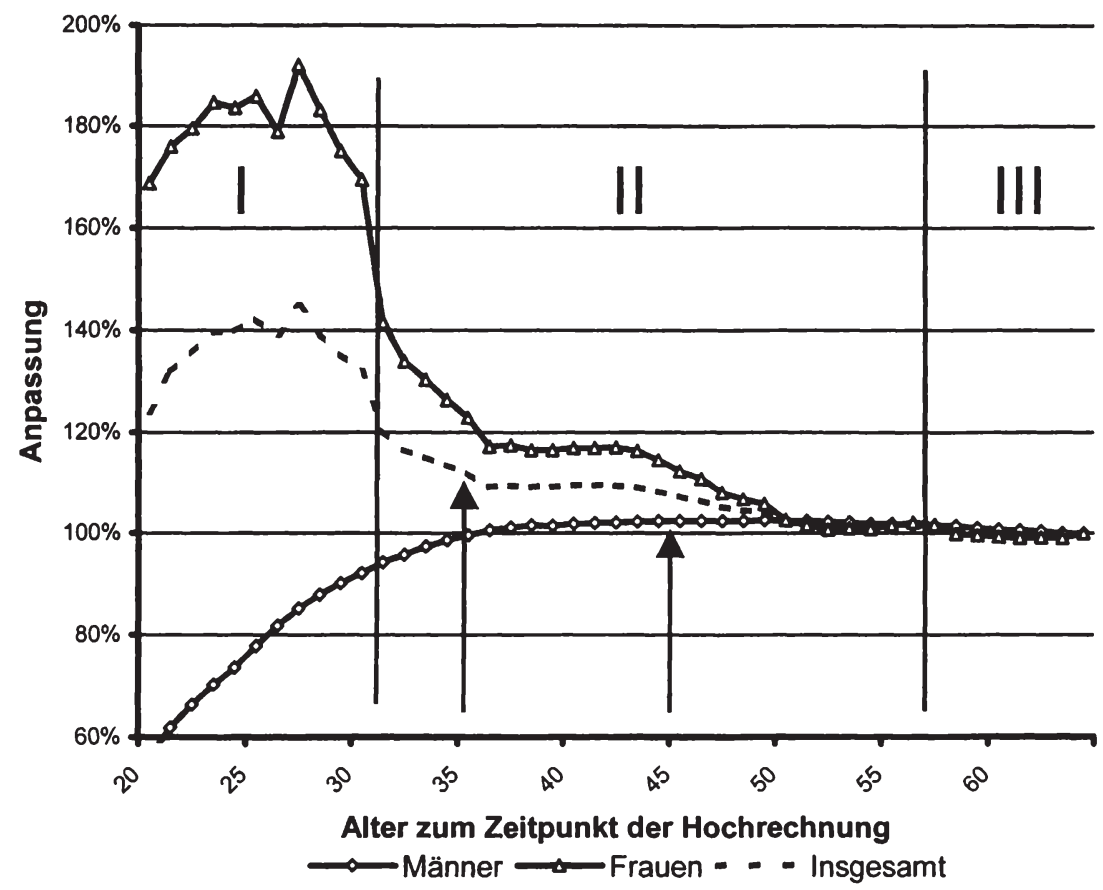

Abbildung 12: Anpassung der Prognosewerte an die tatsächlichen Entgeltpunkte am Beispiel der Geburtskohorte 1934

Zur Bestimmung der entsprechenden Einkommen in einer Bürgerrente werden die Volkseinkommen in Anlehnung an Prognos (1998) mit einem Wachstum in Höhe von 3 v.H. p.a. fortgeschrieben. Die Rentenlücke zum Zeitpunkt des Renteneintritts ergibt sich dann aus Gleichung (14). 


\subsubsection{Abweichen von einer Normalerwerbsbiographie}

Die Einkommen der Altersrentner werden bekanntlich durch den Verlauf der individuellen Erwerbsbiographie bestimmt. Während dieser Zusammenhang in der gesetzlichen Rentenversicherung in der Rentenformel klar zum Ausdruck kommt, besteht eine derartige Verknüpfung in einer Bürgerrente nur mittelbar, da nur die Einkommen aus der privaten Vorsorge den Einwirkungen der Erwerbsbiographie unterliegen. Die Bürgerrente ist eine per definitionem pro Kopf einheitliche Leistung.

Zur Beschreibung der Auswirkungen und der damit verbundenen Anreize werden vor der Präsentation der empirischen Ergebnisse die Wirkungen eines temporären Abweichens ${ }^{162}$ von einer Normalerwerbsbiographie auf die Einkommen der Altersrentner untersucht.

Hierzu wird die Erwerbsbiographie eines stilisierten Standardrentners $s$ betrachtet, der prinzipiell vom 15. bis zum 64. Lebensjahr eine Vollzeitbeschäftigung ausübt. Zur Beschreibung der Folgen eines temporären Abweichens von einer solchen Normalerwerbsbiographie wird zu einem beliebigen Lebensalter $15 \leq a<65$ die Ausübung einer sonstigen Tätigkeit angenommen. Die resultierenden Wirkungen auf die Gesamteinkommen eines Altersrentners beschreiben dann den Preis einer temporären Abweichung von einer Normalerwerbsbiographie in einem Rentensystem.

\section{Die gesetzliche Rentenversicherung}

In der GRV führt ein temporäres Abweichen von einer Normalerwerbsbiographie zu einer Variation der persönlichen Entgeltpunkte $E P_{s, K}^{(a)}$, die einem Standardrentner $s$ einer Kohorte $K$ in einem Lebensalter von $a$ angerechnet werden.

Vor dem Hintergrund der in Tabelle 11 festgelegten Bewertungsgrundsätze bewirkt eine schulische oder berufliche Bildungsmaßnahme nach Vollendung des 17. Lebensjahres oder die Ausübung eines Wehr- oder Zivildienstes eine Veränderung $\Delta E P_{s, K, \lambda_{1}}^{(a)}$ der persönlichen Entgeltpunkte in Höhe von

162 Hier die vorübergehende Ausübung einer Tätigkeit $\lambda \in\{1,2,3,5,6,7,9\}$. Vgl. Tabelle 9. 


$$
\Delta E P_{s, K, \lambda_{t}}^{(a)}=0,75-\frac{\mathrm{Y}_{t}^{(a)}}{\mathrm{Y}_{t}}: \lambda_{t} \in\{1,2,3\}, t=K+a, a \geq 17 .{ }^{163}
$$

Unter Bezugnahme auf den durchschnittlichen Einkommensverlauf eines typischen Individuums in Abbildung 11 führt dies bei einem Lebensalter von bis zu 21 Jahren zu einer Steigerung der gesetzlichen Rentenansprüche. Aufgrund des steilen Anstiegs der $Y_{t}^{(a)}$ ergeben sich für alle späteren Zeitpunkte innerhalb einer Erwerbsbiographie Einbußen.

Eine temporäre Ausübung einer Teilzeiterwerbstätigkeit $\left(\lambda_{t}=5\right)$ oder einer Arbeitslosigkeit $\left(\lambda_{t}=6\right)$ bedeutet eine Veränderung der persönlichen Entgeltpunkte in Höhe von

$$
\Delta E P_{s, K, \lambda_{t}}^{(a)}=0,5 \cdot \frac{\mathrm{Y}_{t}^{(a)}}{\mathrm{Y}_{t}}: \lambda_{t}=5, t=K+a, a \geq 17
$$

beziehungsweise

$$
\Delta E P_{s, K, \lambda_{t}}^{(a)}=0,2 \cdot \frac{\mathrm{Y}_{t}^{(a)}}{\mathrm{Y}_{t}}: \lambda_{t}=6, t=K+a, a \geq 17 .
$$

Die Rentenansprüche verringern sich also generell. Das Ausmaß der Reduktion wird vom Lebensalter, zu dem eine Tätigkeit $\lambda_{t}=5$ oder $\lambda_{t}=6$ ausgeübt wird, bestimmt.

Ähnliches gilt auch bei der Ausübung einer Hausfrauen- oder Hausmannstätigkeit. In diesem Fall reduzieren sich die persönlichen Entgeltpunkte offenkundig um $\frac{Y_{t}^{(a)}}{Y_{t}}$.

Zur Beschreibung der Einkommenswirkungen einer Kindeserziehung ist zu unterscheiden, ob die Erwerbstätigkeit über den Mutterschaftsurlaub hinaus unterbrochen wird. Falls innerhalb der Kindererziehungszeiten, das sind die ersten drei Jahre nach der Geburt des Kindes, keine Erwerbstätigkeit ausgeübt wird, folgen die $\Delta E P_{s, K, \lambda,}^{(a)}$ aus: $:^{164}$

\footnotetext{
163 Vgl. Kapitel 4.2.1 oder Fußnote 159.

164 Vgl. hierzu Fußnote 149, S. 93.
} 


$$
\Delta E P_{s, K, \lambda_{t}}^{(a)}=3-\sum_{a^{\prime}=a}^{a+2} \frac{Y_{K+a^{\prime}}^{\left(a^{\prime}\right)}}{Y_{K+a^{\prime}}}: \lambda_{t}=9, t=K+a^{\prime}, a^{\prime} \geq 17
$$

Die Wirkung auf die Renteneinkommen wird dabei maßgeblich durch das Lebensalter $a^{\prime}$, zu dem die Kindererziehungszeit beginnt, determiniert. Während sich aufgrund des angenommenen Einkommensverlaufs in jungen Jahren signifikante Einkommensverbesserungen ergeben, führt eine Kindererziehung nach dem vollendeten 29. Lebensjahr trotz der Kompensationsregelungen zu geringeren Ansprüchen. Falls die Berufstätigkeit nicht über die Zeit des Mutterschutzes hinaus unterbrochen wird, ist klar, dass eine Kindererziehung die Rentenansprüche deutlich erhöht. Vor dem Hintergrund des durchschnittlichen Lebensalters der Mütter zum Zeitpunkt der Geburt des ersten Kindes beinhaltet die gesetzliche Rentenversicherung folglich positive Anreize, Kinder zu erziehen. ${ }^{165}$

Zur Illustration der Wirkungsweisen sind die Folgen einer einjährigen Abweichung von einer Normalerwerbsbiographie auf die Alterseinkommen eines Standardrentners in der Abbildung 13 dargestellt. ${ }^{166}$

Die Darstellung belegt, dass die Ausübung einer sonstigen Tätigkeit anstelle einer Vollzeitbeschäftigung die Alterseinkommen i.d.R. negativ beeinflusst. Infolge des hier unterstellten durchschnittlichen Einkommensverlaufs resultieren Einbußen in einer Bandbreite von 0,4 v.H. bis 1,1 v.H. Deutlich wird auch, dass in der gesetzlichen Rentenversicherung die Ausbildungs- bzw. die Lehrzeiten und die Schwanger- bzw. Mutterschaftszeiten einen besonderen Schutz erfahren. Infolge der Bewertung der Anrechungszeiten mit Entgeltpunkten wirkt die Ausübung einer solchen „Tätigkeit“ für junge Personen rentensteigernd. Die Unterbrechung einer Normalerwerbsbiographie wegen einer Kindererziehung führt beispielsweise im Alter von 23 Jahren zu einer Erhöhung der Ruhestandseinkommen in Höhe von 0,84 v.H.; im Alter von 35 Jahren sind es hingegen Einbußen in Höhe von 0,47 v.H. Ähnliches gilt auch für die Durchführung einer Lehre oder einer Ausbildung. Der Zeitpunkt, ab dem sich Nachteile ergeben, beginnt hier bereits mit dem 22. Lebensjahr.

\footnotetext{
165 Angemerkt sei hier, dass im Zuge des anhaltenden Trends, Kinder erst später zu bekommen, diese Vorteile rückläufig sind.

166 Der hierbei angenommene Einkommensverlauf folgt der Abbildung 11, S. 90.
} 


\section{Wirkungen einer einjährigen Unterbrechung einer Vollzeiterwerbstätigkeit in der GRV}

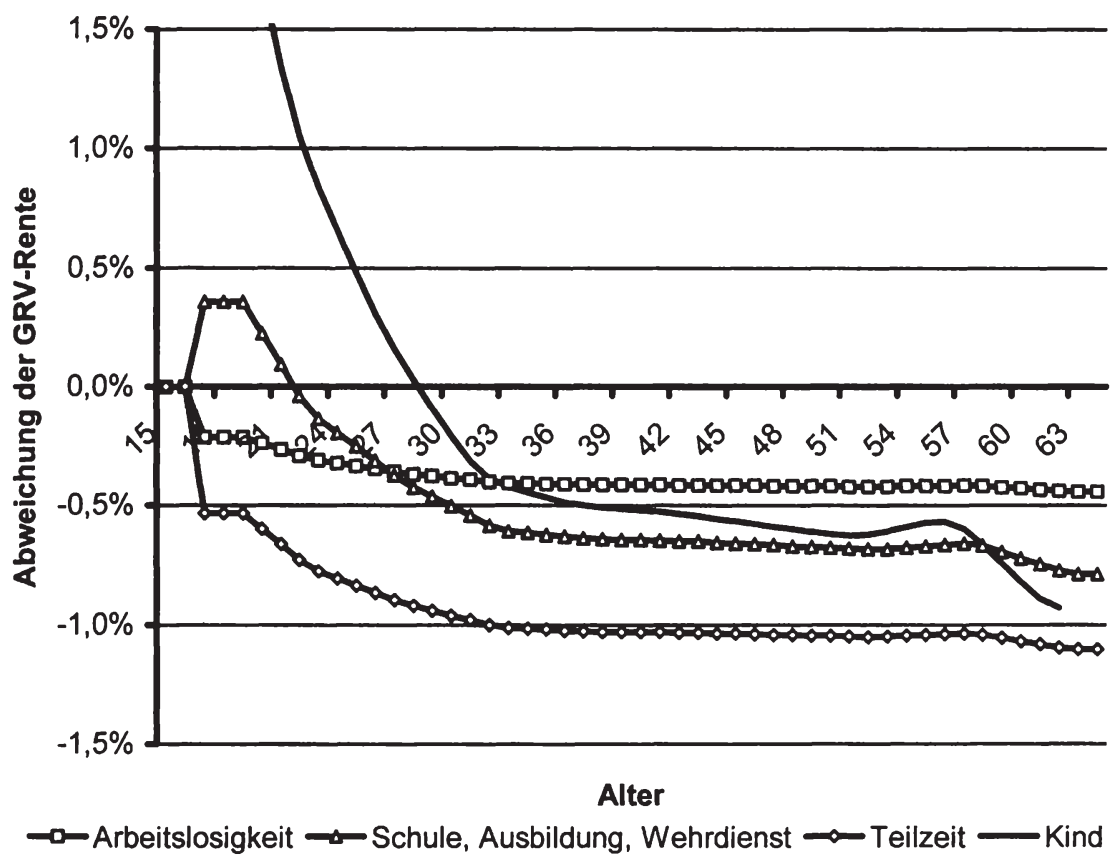

Abbildung 13: Preis eines temporären Abweichens von einer Normalerwerbsbiographie in der GRV $\mathbf{~}^{167}$

\section{Die Bürgerrente}

In der Bürgerrente besitzt ein temporäres Abweichen nur mittelbare Wirkungen auf die Altereinkommen, weil nur die Einkommen aus der eigenständigen Vorsorge $P E_{s, K}^{P R I V}$ den Einwirkungen der Erwerbsbiographie unterliegen. Infolge der hier angenommenen Verknüpfung der Sparfähigkeit mit der Ausübung einer Vollzeitbeschäftigung bedingt ein Abweichen von einer Normalerwerbs-

167 Die angegebenen Daten sind zum Teil nur theoretischer Natur. Dies gilt insbesondere für die Tätigkeiten $\lambda=\{1,2,3,9\}$. 
biographie generell eine Verringerung der Alterseinkommen, da sich die Höhe des möglichen Kapitalstocks $T S_{k, K}$ verringert. Der Betrag, um den sich $T S_{k, K}$ reduziert, ergibt sich mit Gleichung (16) aus:

$$
\Delta T S_{s, K}=\left\{\begin{array}{l}
\sum_{a=0}^{2}\left(S_{s, K}^{(a)} \cdot \prod_{j=a+1}^{a^{-}-1}\left(1+i_{K+j}^{s}\right)\right) \quad \text { falls } \lambda_{K+a}=9 \text { und } a \geq 17 \\
S_{s, K}^{(a)} \cdot \prod_{j=a+1}^{a^{-1}-1}\left(1+i_{K+j}^{s}\right) \quad \text { falls } \lambda_{K+a} \in\{1,2,3,5,6,7\} \text { und } a \geq 17 \\
0 \quad \text { sonst }
\end{array}\right.
$$

Bei einer Kindererziehung wird die Vollzeiterwerbstätigkeit für die Dauer des Mutterschaftsurlaubes, d.h. für drei Jahre unterbrochen. Ansonsten wird die Sparfähigkeit des Individuums nur im Jahr der Ausübung einer sonstigen Tätigkeit ( $\lambda \in\{1,2,3,5,6,7\})$ beeinflusst. Die Verringerung der Gesamtsparleistung und der hieraus resultierenden Einkommen $P E_{s, K}^{P R I V}$ ist offenkundig vom Einkommen zum Zeitpunkt der Unterbrechung und vom Zinsniveau abhängig. Am Beispiel eines Standardrentners der Kohorte 1934 ist die Wirkung auf die Gesamteinkommen im Ruhestand in Abbildung 14 illustriert.

Hierfür sind die Wirkungen auf die Ruhestandseinkommen eines Altersrentners in einer Bürgerrente bei einem Leistungsniveau in Höhe von 50 v.H. (durchgezogene Linien) bzw. 65 v.H. des Volkseinkommens pro Kopf (unterbrochene Linien) abgebildet. Da in einer Bürgerrente eine Bewertung von Anrechnungszeiten nicht erfolgt, sind die Einbußen infolge einer Ausübung einer Tätigkeit $\lambda \in\{1,2,3,5,6,7\}$ (Linien mit Rauten) und infolge einer Kindererziehung (Linien mit Dreiecken) eingezeichnet.

Wird die Betrachtung auf die Folgen einer einjährigen Schul- oder Hochschulzeit, einer Lehre oder Ausbildung, eines Wehr- oder Zivildienstes, einer Teilzeittätigkeit, einer Arbeitslosigkeit oder einer Hausfrauen bzw. -mannstätigkeit konzentriert, verbleiben die Einbußen gegenüber dem Einkommen eines Standardrentners zunächst moderat.

Im Alter von 30 Jahren betragen diese fur ein Leistungsniveau in Höhe von 50 v.H. des Volkseinkommens pro Kopf rund $-0,55$ v.H. Da die Bedeutung der eigenständigen Vorsorge bei höheren Leistungsstufen einer Bürgerrente suk- 
zessive in den Hintergrund tritt, reduziert sich das Gesamteinkommen im Ruhestand bei Leistungen in Höhe von 65 v.H. nur um rund $-0,35$ v.H. Infolge des unterstellten Einkommens- und Zinsverlaufes steigen die Einbußen bei späteren Zeitpunkten einer Unterbrechung an und verharren ab einem Lebensalter von rund 40 Jahren auf einem annähernd konstanten Niveau in Höhe von 1,5 v.H. bzw. 0,9 v.H. des Ruhestandseinkommens eines Standardrentners.

\section{Wirkungen einer einjährigen Unterbrechung einer Vollzeiter- werbstätigkeit in der Bürgerrente}

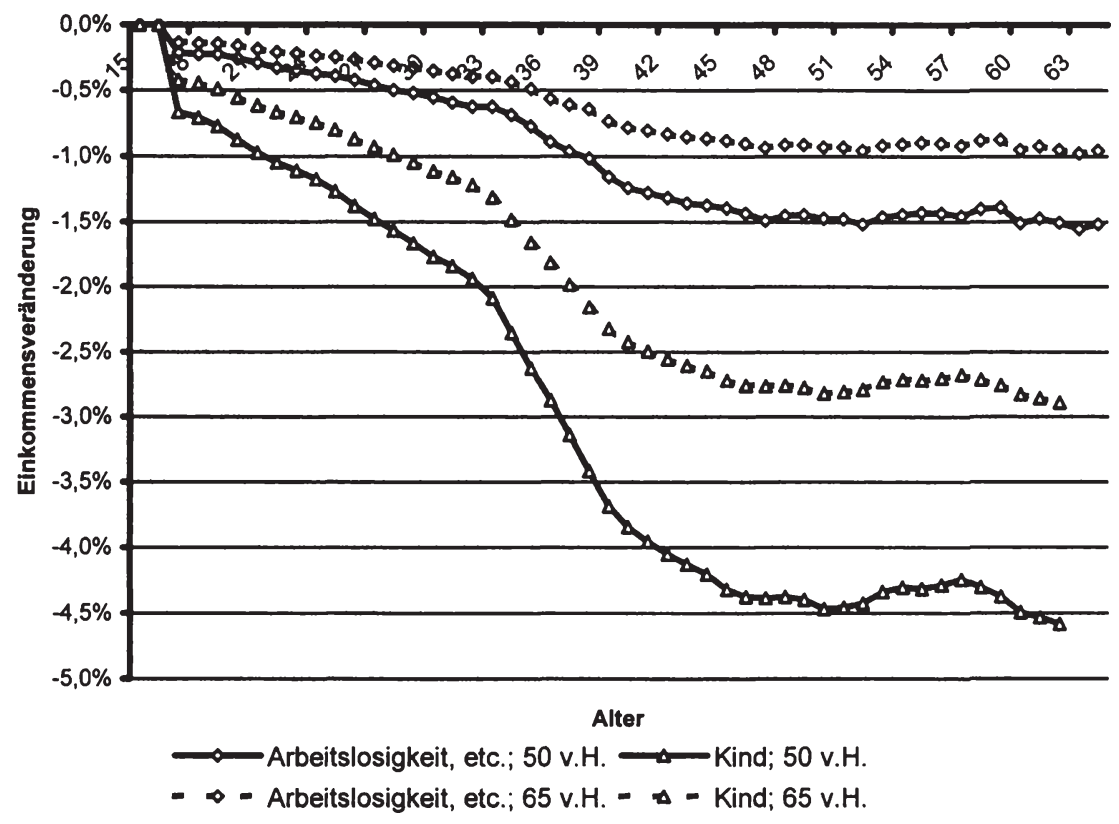

Abbildung 14: Wirkungen eines temporären Abweichens von einer Normalerwerbsbiographie in der Bürgerrente

Bedeutend größer sind die resultierenden Einbußen im Falle einer Unterbrechung aufgrund einer Kindererziehung, da hierfür annahmegemäß die Vollzeiterwerbstätigkeit und damit die Sparfähigkeit für einen Zeitraum von drei Jahren unterbrochen wird. In Ermangelung kompensatorischer Regelungen betragen die Einkommenskürzungen bei Leistungen in Höhe von 50 v.H. des 
Volkseinkommens pro Kopf bis zu 4,5 v.H. Hierbei ist allerdings anzumerken, dass dieser Wert mehr von theoretischer Relevanz ist. Innerhalb des für eine Kindererziehung entscheidenden Lebensalterabschnittes (18 bis 35 Jahren) ergibt sich eine fast lineare Entwicklung der Einkommenseinbußen. Diese betragen in Abhängigkeit vom Zeitpunkt der Unterbrechung zwischen 0,7 v.H. und 2,6 v.H. Für höhere Leistungsstufen einer Bürgerrente sind die Kürzungen entsprechend geringer.

Nach der Analyse der grundsätzlichen Wirkungsweisen biographischer Besonderheiten auf das Gesamteinkommen eines Altersrentners werden im folgenden Teilabschnitt die empirischen Ergebnisse präsentiert, die aus einer Auswertung der SOEP-Daten resultieren.

\subsubsection{Untersuchungsergebnisse}

\subsubsection{Analyse der typischen Personen}

\section{Vollständige Erwerbsbiographien}

Als ein Beispiel vollständiger Erwerbsbiographien wird das erste Cluster der Geburtskohorte 1934 betrachtet, welches 18.8 v.H. des Jahrganges repräsentiert und überwiegend (86,8 v.H.) männlich ist. Die Erwerbsbiographie ist in der Abbildung 10 illustriert.

Die Umstellung der Rentensysteme ist für die typische Person mit einer nachhaltigen Kürzung der Renten aus Systemen der ersten Sicherungssäule verbunden. In Abhängigkeit vom Leistungsniveau einer Bürgerrente beträgt die Rentenlücke bis zu $€ 525$ pro Monat. Werden die aus der Beitragssatzdifferenz resultierenden Beträge zum Zwecke der Altersvorsorge verzinslich angelegt, ergibt sich zum Zeitpunkt des Rentenbeginns das in Tabelle 14 dargestellte Bild.

Die Sparfähigkeit reicht in der Variante ALL/65/ALL18 bei allen betrachteten Leistungsstufen einer Bürgerrente nicht aus, um einen Kapitalstock in Höhe des erforderlichen Kapitals zu akkumulieren (z.B. für Leistungen in Höhe von 50 v.H. des Volkseinkommens ergibt sich: Mögliches Kapital $€ 83993$, Notwendiges Kapital $€$ 92605). Die Person ist also trotz einer langjährigen Vollzeitbeschäftigung und einer damit einhergehenden Sparfähigkeit ein Verlierer einer Systemveränderung. 
Kapitel 4

\section{Einkommen der Altersrentner in einer Bürgerrente (Erstes Cluster der Kohorte 1934)}

\begin{tabular}{|c|c|c|c|c|c|c|c|}
\hline $\begin{array}{l}\text { Leistungs- } \\
\text { niveau }\end{array}$ & Variante & $\mathbf{5 0 , 0 \%}$ & $52,5 \%$ & $55,0 \%$ & $57,5 \%$ & $60,0 \%$ & $65,0 \%$ \\
\hline $\begin{array}{c}\mathrm{PE}_{k . K}^{G A P} \\
\text { Rentenlücke }\end{array}$ & - & 525 & 493 & 461 & 429 & 398 & 335 \\
\hline $\begin{array}{c}T C_{k, K} \\
\text { Notw. } \\
\text { Kapital }\end{array}$ & - & 92605 & 87025 & 81446 & 75867 & 70287 & 59128 \\
\hline$T S_{k, K}^{\left(a^{\circ}\right)}$ & $\begin{array}{l}\text { ALL/65/ } \\
\text { ALL18 }\end{array}$ & 3992 & 78528 & 73063 & 67598 & 62133 & 51204 \\
\hline $\begin{array}{l}\text { Mög. } \\
\text { Kapital }\end{array}$ & $\begin{array}{l}\text { SEL65/ } \\
\text { SEL55 }\end{array}$ & 94349 & 89402 & 84455 & 79508 & 74561 & 64667 \\
\hline \multirow{2}{*}{$\begin{array}{c}D R_{k, K} \\
\text { Bezugs- } \\
\text { dauer }\end{array}$} & $\begin{array}{l}\text { ALL/65/ } \\
\text { ALL18 }\end{array}$ & ,1 & , &, 4 & 3,6 & 3,8 & $-4,3$ \\
\hline & $\begin{array}{l}\text { SEL/65/ } \\
\text { SEL55 }\end{array}$ & 0,7 & 1,0 & 1,3 & 1,7 & 2,2 & 3,5 \\
\hline \multirow{2}{*}{$\begin{array}{c}\mu_{k, K} \\
\text { Anteil an } \\
T S_{k, K}^{\left(a^{*}\right)}\end{array}$} & $\begin{array}{l}\text { ALL/65/ } \\
\text { ALL18 }\end{array}$ & 110,3 & 110,8 & 111,5 & 112,2 & 113,1 & 115,5 \\
\hline & $\begin{array}{l}\text { SEL/65/ } \\
\text { SEL55 }\end{array}$ & 98,2 & 97,3 & 96,4 & 95,4 & 94,3 & 91,4 \\
\hline \multirow{2}{*}{$\begin{array}{c}\pi_{k, K} \\
\text { Einkom- } \\
\text { mens- } \\
\text { quotient }\end{array}$} & $\begin{array}{l}\text { ALL/65/ } \\
\text { ALL18 }\end{array}$ & 95,8 & 95,8 & 95,9 & 96,0 & 96,0 & 96,1 \\
\hline & $\begin{array}{l}\text { SEL/65/ } \\
\text { SEL55 }\end{array}$ & 100,9 & 101,2 & 101,5 & 101,8 & 102,1 & 102,7 \\
\hline
\end{tabular}

Tabelle 14: Einkommen der Altersrentner (Cluster 1, Kohorte 1934)

In der Variante SEL/65/SEL55 ist das Ergebnis erwartungsgemäß positiver. Infolge der geringeren Beitragsbelastung ist die Sparfähigkeit entsprechend größer, so dass trotz der erheblichen Einschnitte bei den Leistungen der ersten Sicherungssäule das Versorgungsniveau der gesetzlichen Rentenversicherung erreicht werden kann. In der Biedenkopf-Miegelschen Ausgestaltung einer 
Bürgerrente zählt diese typische Person also zu den Gewinnern eines Systemwechsels.

Dieses Ergebnis ist auch anhand des Kennzifferkonzepts ersichtlich: Wird wiederum ein Leistungsniveau in Höhe von 50 v.H. betrachtet, dann zeigt eine Bezugdauer in Höhe von z.B. $D R_{1,1934}=-3,1$ in der weitergehenden Variante ALL/65/ALL18, dass die Rentenlücke $P E_{1,1934}^{G A P}$ nur bis ins 81. Lebensjahr geschlossen werden kann. Zur Abdeckung der Mindestbezugsdauer sind Sparbeträge erforderlich, die die mögliche Sparleistung um 10,3 v.H. übersteigen und daher das verfügbare Einkommen in der Erwerbsphase schmälern. Das maximale Einkommen in einer Bürgerrente, das über die Mindestbezugsdauer in konstanten Teilzahlungen bezogen werden kann, erreicht folglich das $\mathrm{Ni}$ veau der gesetzlichen Rente nicht $\left(\pi_{1,1934}=95,8\right)$. Auffällig ist, dass eine Erhöhung des Leistungsniveaus einer Bürgerrente keine wesentliche Verbesserung der Einkommenssituation eines Altersrentner bewirkt. Die mit einer Leistungssteigerung verbundene Anhebung der Beitragsbelastung in einer Bürgerrente führt also zu einer Verdrängung der privaten Vorsorge.

Ein anderes Bild ergibt sich in einer Bürgerrente nach Biedenkopf-Miegel (SEL/65/SEL55): Hier signalisieren die Kennziffern, dass die typische Person des ersten Clusters ein Gewinner einer Systemveränderung ist, wenngleich die Vorteile gering sind. Wird ein Rentenniveau in Höhe von 50 v.H. des Volkseinkommens pro Kopf betrachtet, kann die typische Person ein Einkommen in Höhe der gesetzlichen Rente bis ins 85. Lebensjahr beziehen $\left(D R_{1,1934}=0,7\right)$ bzw. es verbleiben in der Erwerbsphase oder im Alter geringfügig höhere verfügbare Einkommen $\left(\mu_{1,1934}=98,2, \pi_{1,1934}=100,9\right)$ als im bestehenden Alterssicherungssystem. Wenngleich auch hier eine Anhebung der Bürgerrente zu Substitutionseffekten führt, zeigt die Aufstellung, dass die typische Person hiervon zumindest marginal profitieren kann.

Als ein zweites Beispiel dient das dritte Cluster der Geburtskohorte 1934. Diese Gruppe setzt sich annähernd paritätisch aus beiden Geschlechtern zusammen und repräsentiert 33,8 v.H. der Geburtskohorte. ${ }^{168}$ Da der Renteneintritt hier mit dem vollendeten 57. Lebensjahr erfolgt, handelt es sich um ein Beispiel zur Illustration der Wirkungen von Frühverrentungen. Die Ergebnisse der Einkommensanalyse ergeben sich aus Tabelle 15.

Der Verlauf der Erwerbsbiographie ist in der Abbildung 10 dargestellt. 


\section{Einkommen der Altersrentner in einer Bürgerrente (Drittes Cluster der Kohorte 1934)}

\begin{tabular}{cccccccc}
\hline $\begin{array}{c}\text { Leistungs- } \\
\text { niveau }\end{array}$ & Variante & $\mathbf{5 0 , 0 \%}$ & $\mathbf{5 2 , 5 \%}$ & $\mathbf{5 5 , 0 \%}$ & $\mathbf{5 7 , 5 \%}$ & $\mathbf{6 0 , 0 \%}$ & $\mathbf{6 5 , 0 \%}$ \\
\hline $\begin{array}{c}D R_{k, K} \\
\begin{array}{c}\text { Bezugs- } \\
\text { dauer }\end{array}\end{array}$ & $\begin{array}{c}\text { ALL65/ } \\
\text { ALL18 }\end{array}$ & $-11,2$ & $-10,7$ & $-10,0$ & $-9,2$ & $-8,1$ & $-4,5$ \\
& $\begin{array}{c}\text { SEL/65/ } \\
\text { SEL55 }\end{array}$ & $-8,1$ & $-7,0$ & $-5,6$ & $-3,8$ & $-1,3$ & 8,4 \\
\hline$\mu_{k, K}$ & ALL/65/ & 138,2 & 135,2 & 131,8 & 127,8 & 123,1 & 110,8 \\
$\begin{array}{c}\text { Anteil an } \\
T S_{k, K}^{\left(a^{\circ}\right)}\end{array}$ & $\begin{array}{c}\text { SEL/65/ } \\
\text { SEL55 }\end{array}$ & 123,0 & 118,8 & 114,0 & 108,7 & 102,6 & 87,7 \\
\hline $\begin{array}{c}\pi_{k, K} \\
\begin{array}{c}\text { Einkom- } \\
\text { mens- } \\
\text { quotient }\end{array}\end{array}$ & $\begin{array}{c}\text { ALL/65/ } \\
\text { ALL18 }\end{array}$ & 89,8 & 91,2 & 92,6 & 94,0 & 95,4 & 98,2 \\
\hline
\end{tabular}

Tabelle 15: Einkommen der Altersrentner (Cluster 3, Kohorte 1934)

Infolge des frühzeitigen Renteneintritts können die Personen der dritten Gruppe i.d.R. nicht von einer Umstellung der Rentensysteme profitieren.

In der weitergehenden Variante einer Bürgerrente (ALL/65/ALL18) kann die Rentenlücke beispielsweise bei einem Leistungsniveau in Höhe von $50 \mathrm{v}$. H. des Volkseinkommens pro Kopf nur bis ins 73. Lebensjahr $\left(D R_{3,1934}=-11,2\right)$ geschlossen werden. Soll nur die Mindestbezugsdauer gedeckt werden, verfügen die Individuen dieser Gruppe entweder in der Erwerbsphase oder im Ruhestand $\left(\mu_{3,1934}=138,2, \pi_{3,1934}=89,8\right)$ über geringere Einkommen als im System der gesetzlichen Rentenversicherung. Verbesserungen der Finanzsituation ergeben sich nur dann, wenn das Leistungsniveau einer Bürgerrente steigt. Werden z.B. Zahlungen in Höhe von 65 v.H. gewährt, reichen die Einkommen aus eigenständiger Vorsorge bis ins 80 . Lebensjahr, bzw. die typische Person kann in der Erwerbsphase oder im Alter über Einkommen verfügen, die annähernd an das Niveau der Einkommen in der GRV heranreichen. 
Ähnliches gilt auch in der Biedenkopf-Miegelschen Variante SEL/65/SEL55: Während bei geringen und mittleren Leistungsstufen einer Bürgerrente die Sparfähigkeit nicht ausreicht, die Rentenlücke bis zum vollendeten 85 . Lebensjahr zu schließen, zählt die typische Person bei höheren Bürgerrentenzahlungen zu den Gewinnern einer Systemumstellung. Werden beispielsweise Renten in Höhe von 65 v.H. des Volkseinkommens gewährt, kann die typische Person ein Gesamteinkommen in Höhe der gesetzlichen Rente bis ins 92. Lebensjahr beziehen oder in der Erwerbsphase bzw. im Ruhestand über entsprechend höhere Einkommen als im System der gesetzlichen Rentenversicherung verfügen $\left(\mu_{3,1934}=87,7, \pi_{3,1934}=102,5\right)$.

\section{Unvollständige Erwerbsbiographien}

Die Analyse unvollständiger Erwerbsbiographien erfolgt stets zu zwei Zeitpunkten, da hierdurch die Einwirkung der Fortschreibungsregel veranschaulicht werden kann. Im Einzelnen werden die Berechnungen zum Zeitpunkt der Erhebung und des Renteneintritts durchgeführt.

In einem ersten Beispiel wird das achte Cluster der Geburtskohorte 1944 betrachtet. Hierbei handelt es sich um eine Erwerbsbiographie einer fast durchgängig vollzeitbeschäftigten Person. ${ }^{169}$ Die Gruppenmitglieder sind überwiegend männlich (71,9 v.H.) und repräsentieren 23,3 v.H. der Geburtskohorte. Zum Zeitpunkt der Erhebung ergeben sich die in Tabelle 16 zusammengefassten Kennziffern.

Das Finanztableau zeigt ein uneinheitliches Bild. In Variante ALL/65/ALL18 ist die typische Person i.d.R. nicht in der Lage, ausreichend vorzusorgen, d.h. die Rentenlücke kann nicht über den erforderlichen Zeitraum geschlossen werden bzw. die Einkommen in der Erwerbs- oder in der Ruhestandsphase verbleiben unterhalb der entsprechenden Einkommen in der gesetzlichen Rentenversicherung. Während sich für Leistungen in Höhe von 50 v.H. noch marginale Vorteile ergeben, führt bereits ein Rentenniveau in Höhe von 55 v.H. zu Einbußen. Für Leistungen in Höhe von 65 v.H. des Volkseinkommens pro Kopf betragen diese rund 4 v.H. Diese typische Person kann also nicht von höheren Leistungen einer Bürgerrente profitieren. Im Gegenteil: Die Gesamt-

169 Nach einer Ausbildung oder Lehre $(\lambda=2)$ wird ab dem 18. Lebensjahr eine Vollzeitberufstätigkeit ( $\lambda=4$ ) ausgeübt. Der Renteneintritt erfolgt mit 65 Jahren. 
einkommen in einer Bürgerrente sind hier negativ mit dem Leistungsniveau korreliert.

\section{Einkommen der Altersrentner in einer Bürgerrente - Zum Zeitpunkt der Erhebung - (Achtes Cluster der Kohorte 1944)}

\begin{tabular}{|c|c|c|c|c|c|c|c|}
\hline $\begin{array}{l}\text { Leistungs- } \\
\text { niveau }\end{array}$ & Variante & $50,0 \%$ & $52,5 \%$ & $55,0 \%$ & $57,5 \%$ & $60,0 \%$ & $65,0 \%$ \\
\hline \multirow{2}{*}{$\begin{array}{c}D R_{k, K} \\
\text { Bezugs- } \\
\text { dauer }\end{array}$} & $\begin{array}{l}\text { ALL/65/ } \\
\text { ALL18 }\end{array}$ & 0,5 & 0,0 & $-0,5$ & $-1,1$ & $-1,8$ & $-3,3$ \\
\hline & $\begin{array}{l}\text { SEL/65/ } \\
\text { SEL55 }\end{array}$ & 4,7 & 4,6 & 4,5 & 4,4 & 4,2 & 3,9 \\
\hline \multirow{2}{*}{$\begin{array}{c}\mu_{k, K} \\
\text { Anteil an } \\
T S_{k, K}^{\left(a^{\circ}\right)}\end{array}$} & $\begin{array}{l}\text { ALL/65/ } \\
\text { ALL18 }\end{array}$ & 98,3 & 99,9 & 101,7 & 103,9 & 106,3 & 112,9 \\
\hline & $\begin{array}{l}\text { SEL/65/ } \\
\text { SEL55 }\end{array}$ & 87,5 & 87,8 & 88,0 & 88,3 & 88,6 & 89,4 \\
\hline \multirow{2}{*}{$\begin{array}{c}\pi_{k, K} \\
\text { Einkom- } \\
\text { mens- } \\
\text { quotient }\end{array}$} & $\begin{array}{l}\text { ALL/65/ } \\
\text { ALL18 }\end{array}$ & 100,9 & 100,0 & 99,2 & 98,4 & 97,6 & 96,0 \\
\hline & $\begin{array}{l}\text { SEL/65/ } \\
\text { SEL55 }\end{array}$ & 107,1 & 106,6 & 106,1 & 105,6 & 105,1 & 104,2 \\
\hline
\end{tabular}

Tabelle 16: Einkommen der Altersrentner, Finanztableau zum Zeitpunkt der Erhebung (Cluster 8, Kohorte 1944)

Wird hingegen die Biedenkopf-Miegelsche Fassung einer Bürgerrente (Varianten SEL/65/SEL55) betrachtet, zählt die typische Person zu den Gewinnern. Infolge der geringeren Beitragsbelastung kann die Person einen Kapitalstock akkumulieren, durch den die Rentenlücke über das erforderliche Maß hinaus geschlossen werden kann. Alternativ hierzu ist die Person in der Lage, Einkommensverbesserungen zu realisieren. Wie auch in der weitergehenden Variante zeigt sich, dass für diese typische Person geringe Leistungen einer Bürgerrente vorteilhafter sind.

In einem weiteren Analyseschritt wird die finanzielle Situation zum Zeitpunkt des Eintritts in den Ruhestand untersucht. Die Ergebnisse sind in der Tabelle 17 zusammengefasst. 


\section{Einkommen der Altersrentner in einer Bürgerrente - Zum Zeitpunkt des Eintritts in den Ruhestand - (Achtes Cluster der Kohorte 1944)}

\begin{tabular}{|c|c|c|c|c|c|c|c|}
\hline $\begin{array}{l}\text { Leistungs- } \\
\text { niveau }\end{array}$ & Variante & $50,0 \%$ & $52,5 \%$ & $55,0 \%$ & $57,5 \%$ & $60,0 \%$ & $65,0 \%$ \\
\hline \multirow{2}{*}{$\begin{array}{c}D R_{k, K} \\
\text { Bezugs- } \\
\text { dauer }\end{array}$} & $\begin{array}{l}\text { ALL/65/ } \\
\text { ALL18 }\end{array}$ & 7,1 & 6,5 & 5,8 & 5,0 & 4,1 & 2,2 \\
\hline & $\begin{array}{l}\text { SEL/65/ } \\
\text { SEL55 }\end{array}$ & 13,94 & 13,9 & 13,9 & 13,8 & 13,8 & 13,7 \\
\hline \multirow{2}{*}{$\begin{array}{c}\mu_{k, K} \\
\text { Anteil an } \\
T S_{k, K}^{\left(a^{*}\right)}\end{array}$} & $\begin{array}{l}\text { ALL/65/ } \\
\text { ALL18 }\end{array}$ & 83,0 & 84,1 & 85,5 & 87,0 & 88,8 & 93,6 \\
\hline & $\begin{array}{l}\text { SEL/65/ } \\
\text { SEL55 }\end{array}$ & 73,9 & 73,9 & 73,9 & 74,0 & 74,0 & 74,1 \\
\hline \multirow{2}{*}{$\begin{array}{c}\pi_{k, K} \\
\text { Einkom- } \\
\text { mens- } \\
\text { quotient }\end{array}$} & $\begin{array}{l}\text { ALL/65/ } \\
\text { ALL18 }\end{array}$ & 110,1 & 108,8 & 107,5 & 106,2 & 104,9 & 102,3 \\
\hline & $\begin{array}{l}\text { SEL/65/ } \\
\text { SEL55 }\end{array}$ & 117,3 & 116,4 & 115,5 & 114,5 & 113,6 & 111,8 \\
\hline
\end{tabular}

Tabelle 17: Einkommen der Altersrentner, Finanztableau zum Zeitpunkt des Eintritts in den Ruhestand (Cluster 8, Kohorte 1944)

Zum Zeitpunkt des mit 65 Jahren angenommenen Renteneintritts zeigen sich die Einwirkungen der langjährigen Vorsorgefähigkeit: Die typische Person kann in beiden Hauptvarianten von einer Umstellung der Rentensysteme profitieren. In Analogie zum vorherigen Abschnitt bestätigt sich dabei, dass ein geringes Leistungsniveau vorteilhaft sein kann.

Wird zunächst Variante ALL/65/ALL18 betrachtet, kann die Rentenlücke bei einer Bürgerrente in Höhe von 50 v.H. des Volkseinkommens pro Kopf bis ins 91. Lebensjahr geschlossen werden. Alternativ dazu ermöglicht die Systemveränderung der typischen Person ein im Vergleich zu den bestehenden Systemen höheres Gesamteinkommen in der Erwerbsphase $\left(\mu_{k, K}=83,0\right)$ beziehungsweise im Ruhestand $\left(\pi_{k, K}=110,1\right)$. Gewährt die Bürgerrente Renten in Höhe von 65 v.H. des Volkseinkommens, reduziert sich die Sparfähigkeit der typi- 
Kapitel 4

schen Person signifikant, so dass bedeutend geringere Vorteile gegenüber dem Sachstand in der GRV verbleiben $\left(\mu_{k, K}=93,6, \pi_{k, K}=102,3\right)$.

In der Biedenkopf-Miegelschen Ausgestaltung (SEL/65/SEL55) sind die Ergebnisse erwartungsgemäß positiver. In Analogie zur weitergehenden Variante sind die Vorteile gegenüber den bestehenden Systemen bei geringen Leistungsstufen einer Bürgerrente am größten.

Für den Fall einer langjährig vollzeitbeschäftigten Person bestätigt sich also das intuitiv erwartete Ergebnis: Die Umstellung der Rentensysteme auf eine Bürgerrente ist für die typische Person mit Vorteilen verbunden. Diese sind dann maximal, wenn sich das System der Bürgerrente auf die Absicherung existentieller Risiken beschränkt und den Individuen eine größtmögliche Eigenverantwortlichkeit überlässt.

\section{Einkommen der Altersrentner in einer Bürgerrente - Zum Zeitpunkt der Erhebung - (Neuntes Cluster der Kohorte 1944)}

\begin{tabular}{cccccccc}
\hline $\begin{array}{c}\text { Leistungs- } \\
\text { niveau }\end{array}$ & Variante & $\mathbf{5 0 , 0 \%}$ & $\mathbf{5 2 , 5 \%}$ & $\mathbf{5 5 , 0} \%$ & $\mathbf{5 7 , 5 \%}$ & $\mathbf{6 0 , 0 \%}$ & $\mathbf{6 5 , 0 \%}$ \\
\hline $\begin{array}{c}D R_{k, K} \\
\text { Bezugs- } \\
\text { dauer }\end{array}$ & $\begin{array}{c}\text { ALL/65/ } \\
\text { ALL18 }\end{array}$ & 6,0 & 6,6 & 7,3 & 8,3 & 9,4 & 13,0 \\
\hline $\begin{array}{c}\text { SEL/65/ } \\
\text { SEL55 }\end{array}$ & 12,3 & 14,1 & 16,4 & 19,5 & 23,9 & 43,5 \\
\hline $\begin{array}{c}\mu_{k, K} \\
\text { Anteil an } \\
T S_{k, K}^{\left(a^{*}\right)}\end{array}$ & $\begin{array}{c}\text { ALL/65/ } \\
\text { SEL18 }\end{array}$ & 85,0 & 83,9 & 82,6 & 81,2 & 79,4 & 74,9 \\
\hline $\begin{array}{c}\pi_{k, K} \\
\text { SEL55 }\end{array}$ & ALL/65/ & 75,7 & 73,7 & 71,5 & 69,1 & 66,2 & 59,3 \\
$\begin{array}{c}\text { ALL18 } \\
\text { quotiens- }\end{array}$ & SEL/65/ & 106,9 & 107,0 & 107,0 & 107,0 & 107,0 & 107,9 \\
\hline
\end{tabular}

Tabelle 18: Einkommen der Altersrentner, Finanztableau zum Zeitpunkt der Erhebung (Cluster 9, Kohorte 1944) 
Zur Illustration der finanziellen Auswirkungen einer Systemumstellung auf eine Person, die infolge von z.B. beruflichen Veränderungen oder einer Kindererziehung eine Vollzeitbeschäftigung temporär unterbricht, wird exemplarisch das neunte Cluster der Kohorte 1944 betrachtet. Diese Gruppe repräsentiert ein überwiegend weibliches (94,3 v.H.) Profil, welches durch eine kurze Schul- und Ausbildungsphase mit einer sich daran anschließenden Vollzeitbeschäftigung geprägt wird. Vom 30 . bis zum 36. Lebensjahr wird eine Teilzeitbeschäftigung ausgeübt. Die Gruppenmitglieder repräsentieren 8,9 v.H. der Kohorte. Zum Zeitpunkt der Erhebung ergeben sich die in Tabelle 18 zusammengestellten Kennziffern.

Auf der Grundlage der Daten der Teilbiographie ist die typische Person trotz der Unterbrechung der Vollzeiterwerbstätigkeit und einer damit einhergehenden temporären Sparunfähigkeit ein Gewinner der Bürgerrente.

In der Variante ALL/65/ALL18 kann die typische Person die Rentenlücke gut sechs Jahre länger als erforderlich schließen oder höhere verfügbare Einkommen in der Erwerbsphase erzielen. Bei einem Rentenniveau einer Bürgerrente in Höhe von 50 v.H. des Volkseinkommens pro Kopf genügen beispielsweise 85,0 v.H. der möglichen Sparfähigkeit zur Akkumulation des erforderlichen Kapitalstocks. Alternativ dazu kann ein Ruhestandseinkommen erreicht werden, das die zu vergleichende gesetzliche Rente um 6,9 v.H. übersteigt. Für höhere Leistungsstufen einer Bürgerrente weiten sich die Vorteile aus. Im Unterschied zu einem durchgängig Beschäftigten zeigt sich bei einer Person mit einer unterbrochenen Erwerbsbiographie, dass ein erhöhtes Leistungsniveau einer Bürgerrente positiv wirken kann. Ein entscheidendes Kriterium hierfür ist - wie intuitiv leicht nachvollziehbar - die Anzahl der in der gesetzlichen Rentenversicherung erreichten Rentenpunkte und im Hinblick auf die Sparfähigkeit eines Individuums die Dauer einer Vollzeiterwerbstätigkeit in Relation zu der Dauer sonstiger Tätigkeiten. Ist die Anzahl der Entgeltpunkte gering und damit meist einhergehend die Dauer einer Vollzeiterwerbstätigkeit kurz, ist ein gesteigertes Bürgerrentenniveau mit Vorteilen für die typische Person verbunden. Ein ähnliches Bild ergibt sich auch in der Variante SEL/65/SEL55. Infolge der geringeren Belastungen sind die Vorteile hier größer.

Zum Zeitpunkt des Renteneintritts bestätigt sich im Wesentlichen das bekannte Bild: Die typische Person des neunten Clusters zählt in beiden Hauptausgestaltungen zu den Gewinnern einer Systemumstellung. Die finanzielle Situation zum Zeitpunkt des Ruhestandeintritts ergibt sich aus der Tabelle 19. 


\section{Einkommen der Altersrentner in einer Bürgerrente \\ - Zum Zeitpunkt des Eintritts in den Ruhestand - (Neuntes Cluster der Kohorte 1944)}

\begin{tabular}{cccccccc}
\hline $\begin{array}{c}\text { Leistungs- } \\
\text { niveau }\end{array}$ & Variante & $\mathbf{5 0 , 0 \%}$ & $\mathbf{5 2 , 5 \%}$ & $\mathbf{5 5 , 0 \%}$ & $\mathbf{5 7 , 5 \%}$ & $\mathbf{6 0 , 0 \%}$ & $\mathbf{6 5 , 0 \%}$ \\
\hline $\begin{array}{c}D R_{k, K} \\
\begin{array}{c}\text { Bezugs- } \\
\text { dauer }\end{array}\end{array}$ & $\begin{array}{c}\text { ALL/65/ } \\
\text { ALL18 }\end{array}$ & 16,0 & 16,9 & 18,0 & 19,4 & 21,2 & 27,1 \\
& $\begin{array}{c}\text { SEL/65/ } \\
\text { SEL55 }\end{array}$ & 28,3 & 32,2 & 37,7 & 58,7 & 63,1 & $\infty$ \\
\hline $\begin{array}{c}\mu_{k, K} \\
\text { Anteil an } \\
T S_{k, K}^{\left(a^{\circ}\right)}\end{array}$ & $\begin{array}{c}\text { ALL65/ } \\
\text { ALL18 }\end{array}$ & 71,9 & 71,0 & 70,2 & 69,1 & 67,8 & 64,5 \\
\hline $\begin{array}{c}\text { SEL/65/ } \\
\pi_{k, K}\end{array}$ & SEL55 & 64,0 & 62,4 & 60,7 & 58,7 & 56,5 & 51,1 \\
$\begin{array}{c}\text { Einkom- } \\
\text { mens- } \\
\text { quotient }\end{array}$ & $\begin{array}{c}\text { ALL18 } \\
\text { SEL/65/ }\end{array}$ & 115,6 & 115,0 & 114,4 & 113,8 & 113,3 & 112,0 \\
\hline
\end{tabular}

Tabelle 19: Einkommen der Altersrentner, Finanztableau zum Zeitpunkt des Eintritts in den Ruhestand (Cluster 9, Kohorte 1944)

In der Variante ALL/65/ALL18 kann die Rentenlücke bei Leistungen in Höhe von 50 v.H. pro Kopf bis ins 100 . Lebensjahr geschlossen werden beziehungsweise es können erhebliche Einkommensvorteile $\left(\mu_{k, K}=71,9, \pi_{k, K}=115,6\right)$ realisiert werden.

Ähnliches gilt auch im Falle der Biedenkopf-Miegelschen Ausgestaltung (SEL/ 65/SEL55). Infolge der geringeren Beitragsbelastungen sind die Vorteile entsprechend höher $\left(D R_{k, K}=28,3, \mu_{k, K}=64,0, \pi_{k, K}=122,5\right)$. Im Unterschied zur Situation zum Zeitpunkt der Systemumstellung kann die typische Person von einem erhöhten Leistungsniveau einer Bürgerrente hier nicht profitieren. Werden beispielsweise Renten in Höhe von 65 v.H. des Volkseinkommens gewährt, sinken die Einkommensquotienten in der weitergehenden Fassung auf $\pi_{k, K}=112,0$ bzw. in der Biedenkopf-Miegelschen Ausgestaltung einer Bürgerrente auf $\pi_{k, K}=120,9$. Begründet ist dies in der Formulierung der Fort- 
schreibungsregel, die in der Zeit bis zum Renteneintritt eine Vollzeitberufstätigkeit und damit eine Fähigkeit zum Sparen annimmt.

Die Analyse der finanziellen Situation zum Zeitpunkt des Renteneintritts belegt, dass auch Personen mit unterbrochenen Erwerbsbiographien in der Bürgerrente Vorteile erfahren können.

\subsubsection{Gewinner und Verlierer eines Systemwechsels}

\section{Gewinner-Verlierer-Verhältnis}

Der Vorabschnitt konzentriert sich auf die Analyse der Finanzsituation einer als typisch identifizierten Person. Um ein Gewinner-Verlierer-Verhältnis ermitteln zu können, wird die Analyse auf alle Befragungspersonen einer Kohorte ausgedehnt.

Zur Integration zentraler Leistungsmerkmale der bestehenden Systeme, hier vor allem der gesetzlichen Rentenversicherung, wird grundsätzlich die durchschnittliche Kinderzahl der Individuen einer Geburtskohorte bei der Bestimmung der rentenrechtlichen Entgeltpunkte berücksichtigt. ${ }^{170}$ Die Identifikation eines Gewinners bzw. eines Verlierers einer Systemveränderung erfolgt durch die Anwendung des im Abschnitt 4.2 eingeführten Kennziffernkonzepts. Dementsprechend ist eine Person $i$ einer Kohorte $K$ ein Gewinner (Verlierer), falls für den Einkommensquotienten $\pi_{i, K}>1\left(\pi_{i, K}<1\right)$ gilt.

Das Gewinner-Verlierer-Verhältnis ist in der Abbildung 15 am Beispiel der weitergehenden Variante einer Bürgerrente für die Kohorte 1934 illustriert.

In der Darstellung werden die Gewinner einer Systemveränderung für unterschiedliche Leistungsstufen einer Bürgerrente (50 v.H. bis 65 v.H. des Volkseinkommens pro Kopf) angegeben. Dies erfolgt einerseits in einer Säulendarstellung differenziert nach der Höhe der gesetzlichen Rente und andererseits in einer Liniendarstellung, die den Prozentsatz der Gewinner bei einer bestimmten Leistungsstufe einer Bürgerrente insgesamt angibt.

170 Hierdurch werden die gesetzlichen Renten tendenziell überschätzt, weil der Mutter und dem Vater die persönlichen Entgeltpunkte aus der Bewertung der Kindererziehungszeiten gutgeschrieben werden können. 


\section{Anteil der Gewinner in der Kohorte 1934}

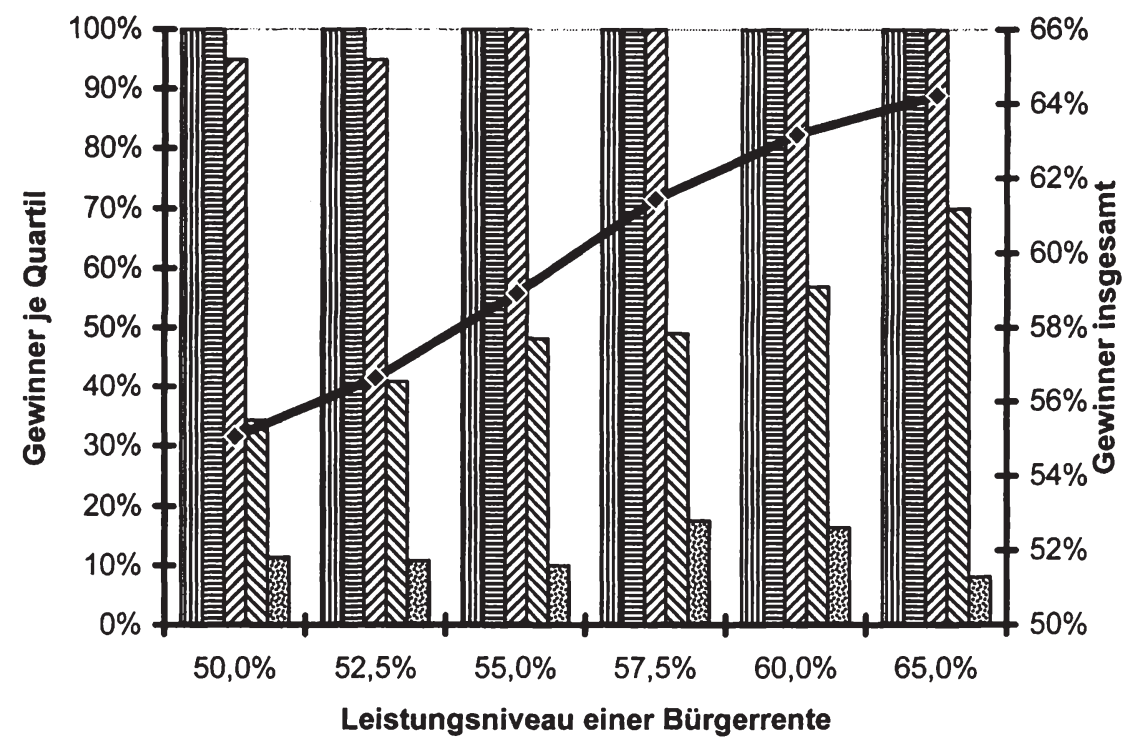

\footnotetext{
메메III) $<=25 \%$

ए $>25 \%$ bis $<=50 \%$

Em $>50 \%$ bis $<=75 \%$

and $>75 \%$ bis $<=100 \%$

Fis. $>100 \%$

$\leadsto-$ Gesamt
}

Anteile der Teilgruppen in der Kohorte:
[I] $13,2 \%$
日 $18,6 \%$
$11,7 \%$
$\boldsymbol{\Delta} 27,0 \%$
国 $30,1 \%$

Abbildung 15: Einkommen und Anteil der Gewinner in der Kohorte 1934

Die Säulendarstellung unterscheidet zwischen Personengruppen, die über unterschiedlich hohe Renten in der gesetzlichen Rentenversicherung verfügen können. Die Höhe der gesetzlichen Renten wird dabei in Relation zum Eckrentenniveau angegeben, welches in Zahlen des Jahres 1999 zu einer Rente in Höhe von netto rund $€ 1028$ führt. Die horizontal gestreifte Säule beinhaltet beispielsweise Personen, deren Rentenansprüche unterhalb von 25 v.H. der Eckrente verbleiben, die gepunktete Säule umfasst hingegen Personen mit Ansprüchen, die die Eckrente übersteigen. Kurzum, die Säulendarstellung differenziert zwischen Quartilen des Eckrentenniveaus. 
Für eine Bürgerrente in Höhe von 50 v.H. des Volkseinkommens pro Kopf ergibt sich beispielsweise, dass - wie intuitiv leicht nachvollziehbar - alle Personen mit geringen Rentenansprüchen, hier mit Ansprüchen in Höhe von bis zu 50 v.H. des Eckrentenniveaus, zu den Gewinnern einer Systemveränderung zählen. Darüber hinaus können auch 34,4 v.H. der Personen mit gesetzlichen Rentenansprüchen in Höhe von über 75 v.H. bis unter 100 v.H. der Eckrente und immerhin 11,4 v.H. der Personen mit Ansprüchen oberhalb des Eckrentenniveaus von einer Systemveränderung profitieren. Neben den Kleinrentnern können also auch Personen mit mittleren und in Teilen auch Personen mit hohen Rentenansprüchen zu den Gewinnern zählen.

Aus der Liniendarstellung ergibt sich, dass eine Umstellung des Rentensystems auf eine Bürgerrente mit Leistungen in Höhe von 50 v.H. des Volkseinkommens pro Kopf für einen überwiegenden Anteil der Individuen $(55,0$ v.H) in der Kohorte 1934 mit Vorteilen verbunden ist.

Werden andere Leistungsstufen einer Bürgerrente angenommen, bestätigt sich der intuitiv erwartete Zusammenhang: Der Anteil der Gewinner ist positiv mit dem Leistungsniveau einer Bürgerrente korreliert.

Erhöht sich die Bürgerrente beispielsweise auf 65 v.H. des Volkseinkommens pro Kopf, können hiervon vor allem Personen mit geringen gesetzlichen Rentenansprüchen profitieren, so dass der Anteil der Gewinner auf 64,2 v.H. ansteigt. Wenngleich die Analyse typischer Erwerbsbiographien zeigt, dass höhere Leistungen aufgrund der damit verbundenen zusätzlichen Beitragsbelastungen im Einzelfall nachteilig wirken können, ergeben sich im Durchschnitt positive, d.h. einkommenssteigernde Effekte. Eine Anhebung der obligatorischen Leistungen einer Bürgerrente bedeutet also vornehmlich eine Besserstellung der Kleinrentner.

Als Beleg für diesen Zusammenhang sind in der Abbildung 16 die aus einer Systemumstellung resultierenden Einkommensveränderungen in einem Diagramm am Beispiel der weitergehenden Varianten für die Kohorte 1934 aufgetragen. Im Einzelnen sind die Wirkungen auf die Einkommen der Altersrentner für Leistungen einer Bürgerrente in einer Bandbreite von 50 v.H. bis 65 v.H. des Volkseinkommen abgebildet. Zur Messung der Einkommensveränderungen wird der durchschnittliche Einkommensquotient bestimmt. In Fortführung einer nach Einkommensklassen gegliederten Betrachtung sind die durchschnittlichen Einkommensquotienten klassifiziert angegeben. Hierbei beschreiben die Linien die Veränderung des Ruhestandseinkommens der Personen mit gesetz- 
lichen Rentenrentenansprüchen in Höhe von bis zu 25 v.H. der Eckrente (Linie mit Rauten) bis über 100 v.H. der Eckrente (Linie mit Dreiecken). Personen mit geringen Rentenansprüchen sind - wie erwartet - die Hauptgewinner einer Systemveränderung. Für Leistungen einer Bürgerrente in Höhe von 50 v.H. ergeben sich für diese Gruppe Vorteile in Höhe von durchschnittlich 342 v.H. Steigen die obligatorischen Leistungen weiter an, erhöhen sich offenkundig auch die Vorteile für die Mitglieder dieser Gruppe. Im Extrem, d.h. für eine Bürgerrente in Höhe von 65 v.H. des Volkseinkommens pro Kopf erreicht der durchschnittliche Einkommensquotient einen Wert in Höhe von 438 v.H. Die Steigung der Einkommensquotientenkurve ist also positiv.

\section{Einkommensveränderungen in der Kohorte 1934}

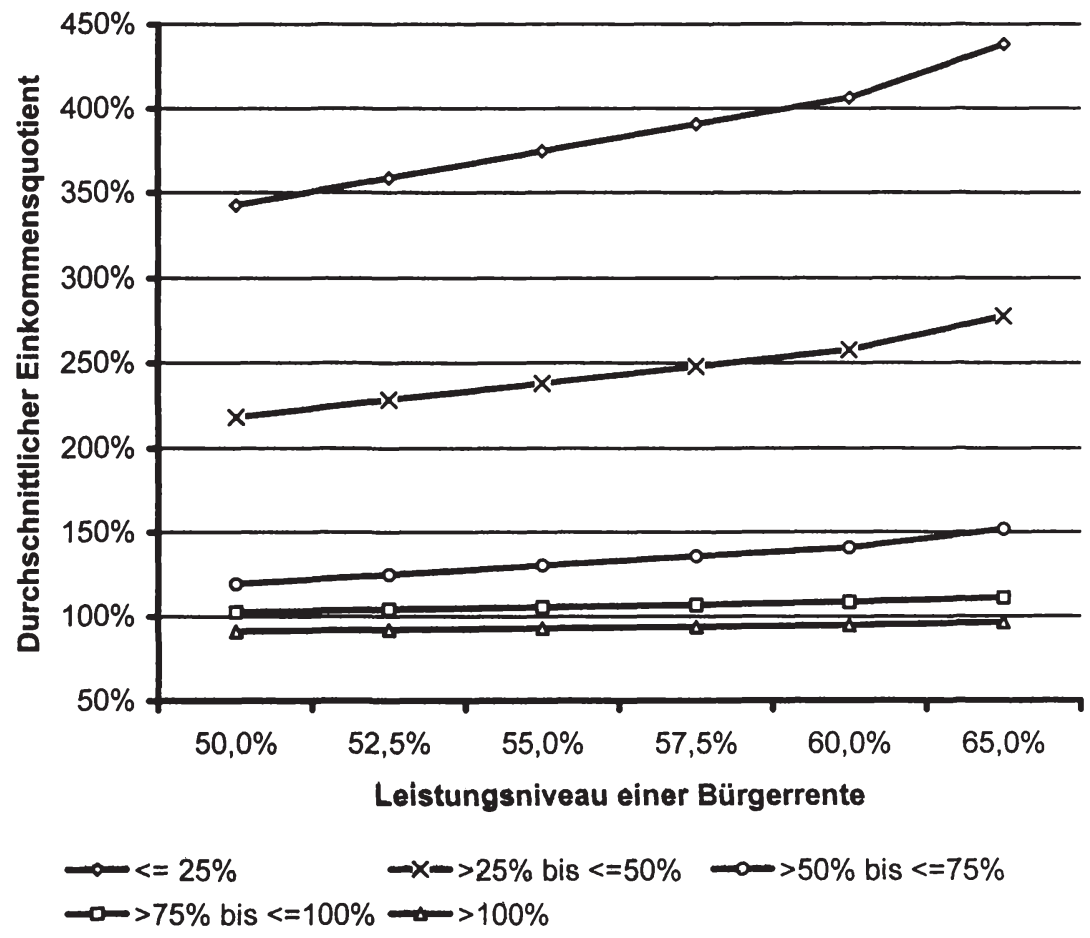

Abbildung 16: Einkommensveränderungen in der Kohorte 1934 
Werden Personengruppen mit höheren Rentenansprüchen betrachtet, ergeben sich geringere Werte für $\pi_{i, K}$. Bei einem Leistungsniveau in Höhe von 50 v.H. des Volkseinkommens pro Kopf können beispielsweise Personen mit Rentenansprüchen in Höhe von 75 v.H. bis unter 100 v.H. der Eckrente (Linie mit Quadraten) noch leichte Verbesserungen erfahren $\left(\pi_{K}=102,5\right)$, während sich Personen mit Rentenansprüchen oberhalb der Eckrente (Linie mit Dreiecken) schlechter als in den bestehenden Systemen der Alterssicherung stellen. Die Einbußen betragen durchschnittlich rund 9 v.H. der gesetzlichen Rente.

Wenngleich höhere Leistungen einer Bürgerrente auch für diese Gruppen vorteilhaft sind, wird anhand der Abbildung 16 klar, dass die einkommenssteigernden Wirkungen begrenzt bleiben. Eine Anhebung der Bürgerrente von 50 v.H. auf 65 v.H. des Volkseinkommens führt beispielsweise für Personen mit Ansprüchen oberhalb der Eckrente zu einer Einkommensverbesserung in Höhe von nur knapp fünf Prozentpunkten.

Wird anstatt der weitergehenden Variante die Biedenkopf-Miegelsche Ausgestaltung einer Bürgerrente betrachtet, bestätigen sich die aufgezeigten $\mathrm{Zu}$ sammenhänge. Infolge einer geringeren Beitragsbelastung sind die Anteile der Gewinner höher und die durchschnittlichen Einkommensveränderungen gröBer. Einzelheiten ergeben sich aus den Abbildungen 24 und 25 im Anhang.

Die Umstellung der bestehenden Rentensysteme auf eine Bürgerrente ist also für den überwiegenden Anteil der Personen in der Kohorte 1934 mit Vorteilen verbunden. Wie erwartet erfahren hierbei insbesondere Personen mit geringen oder mittleren Rentenansprüchen (bis ca. 75 v.H. der Eckrente) signifikante Steigerungen des Ruhestandseinkommens. Aufgrund einer sukzessiv stärker werdenden Substitution individueller Ersparnis durch solidarische Vorsorge des Rentensystems können Personen, die in den bestehenden Systemen über hohe Rentenansprüche verfügen, nur in einem begrenzten Umfang von einer Anhebung des Leistungsniveaus einer Bürgerrente profitieren.

Wird eine Bürgerrente nach Biedenkopf-Miegel implementiert, können aufgrund der damit verbundenen geringeren Beitragsbelastung tendenziell mehr Personen Vorteile erfahren. 


\section{Die Grenze zwischen Gewinnern und Verlierern}

Nach der Analyse des Gewinner-Verlierer-Verhältnisses wird die Einkommensgrenze ermittelt, ab der ein Gewinner zu einem Verlierer wird. Da diese Grenze maßgeblich durch den Verlauf der individuellen Erwerbsbiographie determiniert wird, sei hierfür ein stilisierter Tätigkeitsverlauf eines Standardrentners $s$ unterstellt.

Dieser übe im 15. und 16. Lebensjahr eine schulische Bildungsmaßnahme und im Anschluss daran eine ununterbrochene Vollzeiterwerbstätigkeit bis zum 64 . Lebensjahr aus. Im Alter von 65 Jahren trete $s$ in den Ruhestand ein. Es wird also eine Biographie zugrundegelegt, die z.B. für einen Arbeiter charakteristisch ist. Zur Integration der Kindererziehungszeiten werden dem Ansatz des Vorabschnittes folgend eine durchschnittliche Kinderanzahl in Höhe von 1,4 Kinder pro Person nach den Grundsätzen des § 56(1) SGB VI bewertet.

Wird in einer Modifikation der Berechnungsgrundlagen ${ }^{171}$ die Bewertung des Tätigkeitsartfaktors $\beta_{4}$ variiert, kann das Einkommensniveau, ab dem ein Gewinner $\left(\pi_{s, K}>1\right)$ zu einem Verlierer $\left(\pi_{s, K}<1\right)$ wird, iterativ bestimmt werden. ${ }^{172}$ Die Erwerbseinkommen $Y_{s, K}^{(a)}$ des betrachteten Standardrentners $s$ in einem Lebensalter $a$ ergeben sich also aus einer parallelen Verschiebung des in Abbildung 11 dargestellten Einkommensverlaufs. Für einen Tätigkeitsartfaktor in Höhe von beispielsweise $\beta_{4}=1$, d.h. der Standardrentner erzielt in jedem Jahr ein Einkommen in Höhe des Durchschnittseinkommens aller sozialversicherungspflichtig Beschäftigten der gleichen Altersklasse, folgen gesetzliche Rentenansprüche in Höhe von 117,47 v.H. der Eckrente. ${ }^{173}$ In Zahlen des Jahres 1999 sind das rund $€ 1207$ netto.

Zur Illustration der Wirkungen veränderter Einkommen in der Erwerbsphase auf die Vorteilhaftigkeit einer Systemumstellung für $s$ werden die resultierenden Ruhestandseinkommen, ausgedrückt in Anteilen der Eckrente und die Einkommensquotienten $\pi_{s, K}$ in einem Diagramm aufgetragen. Für die weitergehende Variante (Linen mit Rauten) bzw. die Biedenkopf-Miegelsche Ausgestaltung (Linien mit Dreiecken) einer Bürgerrente ergibt sich am Beispiel eines

\footnotetext{
171 Vgl. Tabelle 11, S. 92.

172 Beitragsbemessungsgrenzen gem. Anlage 2 und 2b SGB VI werden im Zuge der Betrachtung eines stilisierten Erwerbsverlaufes nicht berücksichtigt.

173 Aufgrund des angenommenen Einkommensverlaufs (vgl. Abbildung 11, S. 90) und der Bewertung der Kindererziehungszeiten resultiert ein Rentenanspruch oberhalb der Eckrente.
} 
Standardrentners der Geburtskohorte 1934 der in der Abbildung 17 dargestellte Zusammenhang.

\section{Wirkung der Erwerbseinkommen auf die Vorteilhaftigkeit einer Bürgerrente am Beispiel eines Standardrentners der Kohorte 1934}

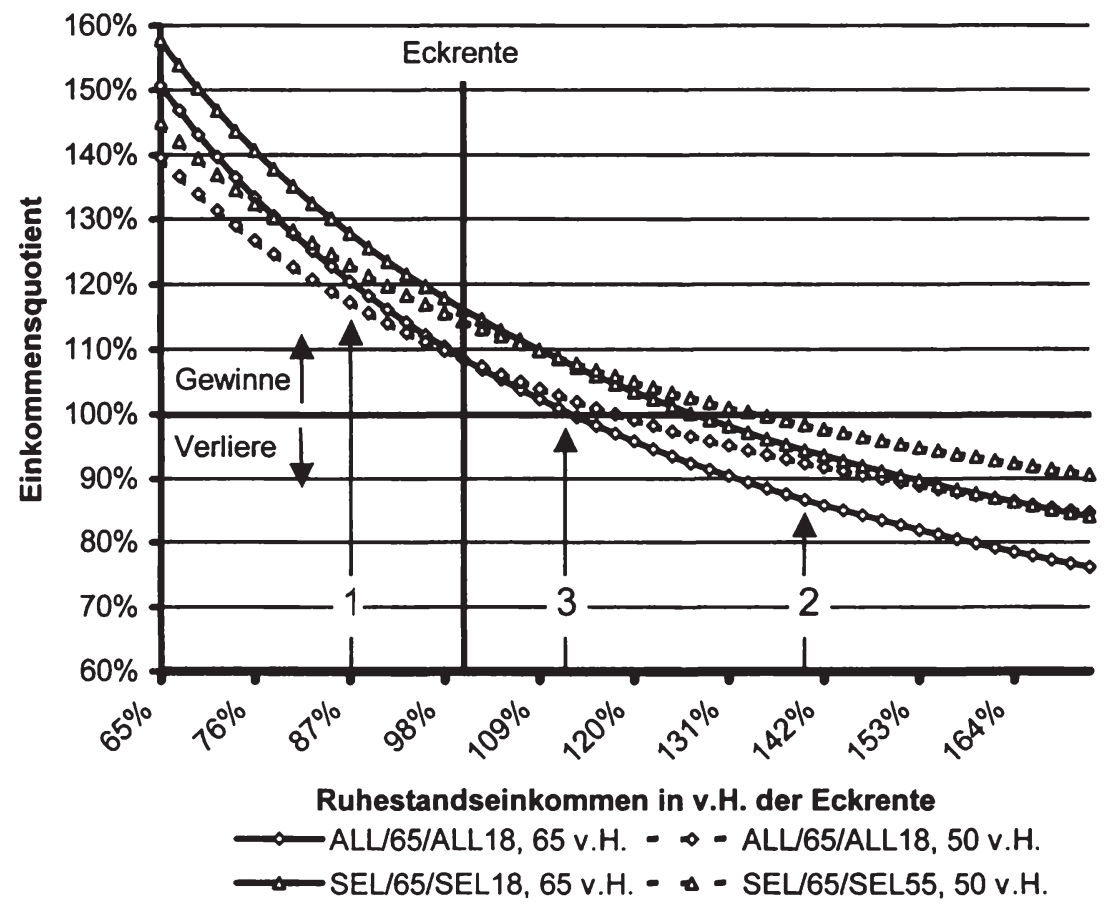

Abbildung 17: Wirkung der Erwerbseinkommen auf die Vorteilhaftigkeit einer Bürgerrente am Beispiel eines Standardrentners der Kohorte 1934

Die Einkommensquotienten als Maßgröße der Vor- bzw. Nachteile sind exemplarisch für Leistungsstufen einer Bürgerrente in Höhe von 50 v.H. (unterbrochene Linien) und 65 v.H. (durchgezogene Linien) des Volkseinkommens 
pro Kopf eingezeichnet, d.h. es wird ein Korridor möglicher Werte für $\pi_{s, 1934}$ durch eine Markierung von Ober- und Untergrenzen angegeben.

Ein Standardrentner, der in der Erwerbsphase stets ein Einkommen in Höhe von 70 v.H. des Durchschnittsentgelts aller sozialversicherungspflichtig Beschäftigten einer Altersklasse erzielt, verfügt in der gesetzlichen Rentenversicherung über ein Ruhestandseinkommen in Höhe von rund 87 v.H. der Eckrente (Markierung Nr. 1). Eine Umstellung des Rentensystems bedeutet für diesen Rentner eine signifikante Besserstellung. In Abhängigkeit vom betrachteten Leistungsniveau einer Bürgerrente übersteigen die Gesamteinkommen im Ruhestand die gesetzliche Rente in der weitergehenden Variante (ALL/65/ALL18) um 17 v.H. bzw. 20 v.H. In der Biedenkopf-Miegelschen Fassung (SEL/65/SEL55) sind die Vorteile mit 23 v.H. bis 28 v.H. entsprechend größer. In einer Bestätigung der wesentlichen Ergebnisse des Vorabschnittes reduzieren sich diese Vorteile bei steigendem Einkommen in der Erwerbsphase. Werden beispielsweise Einkommen in Höhe von 120 v.H. des Durchschnittsentgelts aller Sozialversicherungspflichtigen einer Altersklasse angenommen, folgen hieraus gesetzliche Ansprüche in Höhe von 142 v.H. der Eckrente (Markierung Nr. 2). Eine Systemumstellung führt hier zu Nachteilen. Je nach Höhe der Bürgerrente belaufen sich diese auf 8 v.H. bis 14 v.H. (ALL/65/ALL18) bzw. auf 2 v.H. bis 6 v.H. (SEL/65/SEL55) der gesetzlichen Rente.

Die (Einkommens-)Grenzen, ab der ein Gewinner zu einem Verlierer wird, sind zwischen den Markierungen Eins und Zwei zu finden. In der weitergehenden Fassung einer Bürgerrente liegen diese für den betrachteten Standardrentner je nach Leistungshöhe der Bürgerrente bei Erwerbseinkommen in Höhe von 98 v.H. bzw. 94 v.H. des Durchschnittsentgelts aller sozialversicherungspflichtig Beschäftigten der gleichen Altersklasse bzw. bei Rentenansprüchen in Höhe von rund 118 v.H. bzw. 113 v.H. der Eckrente (Markierung Nr. 3). In der Biedenkopf-Miegelschen Ausgestaltung verläuft die Trennlinie bei Einkommen in Höhe von 114 v.H. bzw. 106 v.H. des Durchschnittsentgelts aller Sozialversicherungspflichtigen einer Altersklasse oder anders ausgedrückt bei Rentenansprüchen in Höhe von 135 v.H. bzw. 127 v.H. der Eckrente. Oberhalb (unterhalb) dieser Grenze handelt es sich um einen Verlierer (Gewinner).

$\mathrm{Zu}$ betonen ist, dass Veränderungen in der Erwerbsbiographie Verschiebungen dieser Grenzen zur Folge haben. Wird anstatt eines Arbeiters beispielsweise eine Erwerbsbiographie eines Akademikers betrachtet, der erst nach Abschluss der Schul- und Studienzeit sowie der Ableistung des Wehr- oder Zivildienstes 
eine Vollzeitbeschäftigung aufnimmt, verlaufen die $\pi_{s, K}$ infolge der geringeren Sparmöglichkeit steiler.

Zur Analyse der mit einem Rentensystem verbundenen Anreizstrukturen werden im folgenden Abschnitt intra- und intergenerationale Umverteilungen in der gesetzlichen Rentenversicherung und in einer Bürgerrente untersucht. Die Analyse konzentriert sich dabei auf die Regelaltersrente als Anspruchsgrundlage in einem Rentensystem.

\subsubsection{Umverteilungen in den Rentensystemen}

Die Umstellung des Sozialsystems auf eine Bürgerrente, d.h. auf ein Versorgungssystem mit einkommensproportionalen Beiträgen und pro Kopf einheitlichen Leistungen, ist inhaltlich eng mit intra- und intergenerationalen Umverteilungen verbunden. ${ }^{174}$ Zur Messung der Umverteilungen wird der grundlegende Ansatz des „Generational Accounting “6175 verwendet. Hiermit können Disparitäten zwischen gezahlten Beiträgen und empfangenen Leistungen einzelner Personen oder ganzer Geburtskohorten und die hieraus resultierenden Anreizstrukturen aufgezeigt werden.

Dem Grundgedanken der Generationenbilanzierung folgend werden die mit dem Preisindex diskontierten Einzahlungen mit den Barwerten der zu erwartenden Leistungen eines Rentensystems $R \in\{B R, G R V\}$ verglichen. ${ }^{176}$ Der Diskontfaktor in einem Jahr $t$ sei $v_{l}$. Der Barwert der Beitragszahlungen $C V_{i, K}^{R}$ einer Person $i$ einer Kohorte $K$ an ein Rentensystem $R$ ergibt sich aus:

$$
C V_{i, K}^{R}=\sum_{a=0}^{A} \mathrm{Y}_{i, K}^{(a)} \cdot \tau_{t}^{R} \cdot v_{t}: t=K+a
$$

Der Barwert der zu erwartenden Rentenzahlungen $P V_{i, K}^{R}$ eines Alterssicherungssystems $R$ an eine Person $i$ einer Kohorte $K$ folgt dementsprechend aus:

174 Zur allgemeinen Analyse der Umverteilungen in den bestehenden Sozialsystemen vgl. Simon (2000).

175 Vgl. z.B. Auerbach et al. (1999) oder Kitterer (1995).

176 Ähnliche Berechnungen finden sich auch z.B. bei Schulenburg (1990), Eitenmüller (1996), Ohsmann, Stolz (1997) oder Glismann, Hom (1998). 


$$
P V_{i, K}^{R}=\sum_{a=0}^{A} P E_{i, K}^{R} \cdot v_{t}
$$

Die Festsetzungen der Parameter folgen dabei zunächst, d.h. bis zum Zeitpunkt der Systemumstellung, den tatsächlichen Daten. Für spätere Zeitpunkte ergeben sich die Einkommen $Y_{i, K}^{(a)}$, die Beitragssätze $\tau_{t}^{R}$ und die Leistungen $P E_{i, K}^{R}$ eines Rentensystems sowie die Diskontfaktoren $v_{t}$ aus dem im Abschnitt 5.1 dargestellten Projektionsmodell.

Falls die Differenz $P V_{i, K}^{R}-C V_{i, K}^{R}$ positiv (negativ) ist, handelt es sich bei der Person $i$ in dem System $R$ um einen Nettoempfänger (Nettozahler) von Transfers. Person $i$ erzielt also eine positive (negative) reale Rendite auf die eingezahlten Beiträge.

Der Verlauf der Salden aus empfangenen Leistungen und entrichteten Beiträgen mehrerer Geburtskohorten $\sum_{i: i \in K} P V_{i, K}^{R}-C V_{i, K}^{R}$ ist dann ein $\mathrm{Ma} ß$ zur Beschreibung der intergenerationalen Redistributionen. Sofern der Saldo einer Kohorte positiv (negativ) ist, werden die Lasten des Rentensystems anderen, i.d.R. nachfolgenden Jahrgängen aufgebürdet (genommen).

\subsubsection{Intragenerationale Umverteilungen}

In der gesetzlichen Rentenversicherung sind die Rentenleistungen infolge des Grundsatzes der Teilhabeäquivalenz im Wesentlichen proportional zu den gezahlten Beiträgen. ${ }^{177}$ Für eine durchgängig vollzeitbeschäftigte Person bedeutet dies, dass die Differenz aus Aus- und Einzahlungen maßgeblich durch externe Faktoren, das sind insbesondere ökonomische und demographische Einflüsse, bestimmt werden. Während eine aufgrund von Produktivitätssteigerungen wachsende Ökonomie infolge einer Bindung der Renten an die Nettolöhne c.p. zu $P V_{i, K}^{R}>C V_{i, K}^{R}$ führt, wirken aktuelle demographische Veränderungen, hier z.B. der Umstand einer alternden Bevölkerung, tendenziell gegenteilig.

Durch das Rentensystem induzierte Umverteilungen und die hieraus abzuleitenden Anreizstrukturen werden in einem solchen Fall nur dann begründet, wenn für einzelne Gruppen von Versicherten die starre Verknüpfung der Beitrags- und der Leistungsseite modifiziert wird. Für die gesetzliche Rentenver- 
sicherung erfolgt dies bekanntlich durch eine Bewertung der rentenrechtlichen Anrechnungszeiten. Hierdurch erfahren Personen, die beispielsweise Ausbildungs-, Wehrdienst- oder Kindererziehungszeiten nachweisen können, eine mitunter signifikante Aufwertung der individuellen Renten, ohne das hierfür eine gesonderte (monetäre) Leistung zu erbringen ist. Es werden folglich intraoder intergenerationale Umverteilungen begründet. Ähnliches gilt im Grundsatz auch für den Umstand der Frühverrentung. Sofern eine Rente abschlagsfrei zu einem früheren Zeitpunkt bezogen werden kann, verringert sich offenkundig die Dauer und damit die Höhe der Einzahlungen. Da sich hierdurch zugleich die Dauer des Rentenbezugs verlängert, werden Umverteilungen begründet. $\mathrm{Ob}$ der im Sozialgesetzbuch festgelegte Abschlag in Höhe von 0,3 v.H. je Monat eines vorzeitigen Bezugs diese Vorteile eliminiert, wird die quantitative Betrachtung zeigen.

Im Unterschied hierzu ist eine Beitragsäquivalenz in einer Bürgerrente i.d.R. nicht gegeben. Ein Rentensystem mit pro-Kopf einheitlicher Rente und lohnproportionalen Beitragsleistungen bedingt grundsätzlich Umverteilungen zwischen den Individuen einer Kohorte. Personen mit geringen oder ohne eigenständige Einkommen zählen offenkundig zu den Empfängern von Nettotransfers, während Personen mit hohen Einkommen mit einer impliziten Steuer belegt werden. ${ }^{178}$ Hierdurch werden offenkundig negative Anreize in Bezug auf die individuelle Arbeitsangebotsentscheidung begründet. Da eine gesonderte Bewertung der Ausbildungs- oder Kindererziehungszeiten nicht erfolgt, sind die hieraus abzuleitenden Anreize offenkundig. Wenngleich der Biedenkopf-Miegelsche Originalentwurf einen vorzeitigen Rentenbezug nicht vorsieht, soll dieser hier modelliert werden, um einerseits eine Vergleichbarkeit zur ermöglichen und andererseits auch, um einen Beitrag zur Bewertung eines Abschlags zu leisten. Hierzu werden die Zahlungen der Bürgerrente als Teilentnahmen aus einem Kapitalstock interpretiert. Bei einer Frühverrentung gilt es nun die Teilentnahmen der verlängerten Laufzeit adäquat anzupassen. Unter der Annahme eines konstanten Kapitalstocks und eines konstanten Kalkulationszinsfußes - hier in Höhe von 4 v.H. - folgen die Abschläge unmittelbar aus der Finanzmathematik.

Die quantitative Analyse der Umverteilungen erfolgt exemplarisch anhand der Kohorte 1934. Hierzu werden die Differenzen $P V_{i, K}^{R}-C V_{i, K}^{R}$ einer Person $i$ auf 
der Grundlage des im Abschnitt 3.2 vorstellten Verfahrens in der gesetzlichen Rentenversicherung und in einer Bürgerrente ermittelt und zur Bewertung der durchschnittlichen Umverteilungen aggregiert. Die Berechnung erfolgt exemplarisch für die weitergehende Variante einer Bürgerrente (ALL/65/ALL18) und ist im Geldwert von 1999 (umgerechnet in Euro) angegeben. Einzelheiten ergeben sich aus der Tabelle 20.

\section{Umverteilungen in den Rentensystemen am Beispiel der Kohorte 1934}

\begin{tabular}{cc}
\hline Rentensystem & Durchschnittlicher Saldo in EUR \\
\hline Gesetzliche Rentenversicherung & 86919 \\
\hline ALL/A65/ALL18, 50 v.H. & 86984 \\
\hline ALL/A65/ALL18, 65 v.H. & 109995 \\
\hline
\end{tabular}

Tabelle 20: Durchschnittlicher Pro-Kopf Saldo am Beispiel der Kohorte 1934

Die resultierenden durchschnittlichen Differenzen sind für die Geburtskohorte 1934 stets positiv, d.h. die Individuen dieser Kohorte sind im Durchschnitt Nettoempfänger von Transferleistungen. Zudem zeigt sich, dass das Ausmaß der Umverteilungen in einer Bürgerrente positiv mit dem Leistungsniveau korreliert ist. Während die Höhe der durchschnittlichen Umverteilungen bei einem Leistungsniveau in Höhe von 50 v.H. des Volkseinkommens pro Kopf ungefähr dem Zahlenwert der gesetzlichen Rentenversicherung entspricht, ergeben sich für ein Leistungsniveau in Höhe von 65 v.H. deutlich höhere Werte. Vor dem Hintergrund, dass ein gesteigertes Leistungsniveau grundsätzlich mit Vorteilen für Personen mit geringen Renten oder im Extrem ohne eigenständige Renten verbunden ist, ist dieses Ergebnis leicht nachvollziehbar.

\subsubsection{Intergenerationale Umverteilungen}

Die Analyse der intergenerationalen Redistributionen erfolgt anhand von drei Referenzszenarien: Ein Standardrentner $s$ mit Kindern, ein Standardrentner ohne Kinder und ein Frührentner. ${ }^{179}$ Hierdurch können die unterschiedlichen

179 Hierbei werden 1,4 Kinder nach den Grundsätzen des $\S 56$ SGB VI bewertet. Eine Unterbrechung der Erwerbstätigkeit erfolgt nicht. 
Bewertungen der Kindererziehungsleistungen und die Folgen einer vorzeitigen Verrentung untersucht werden.

Ein Standardrentner sei hierbei durch eine ununterbrochene Erwerbsbiographie von 20. bis zum 64. Lebensjahr charakterisiert. Im Unterschied hierzu ist ein Frührentner eine Person, die bereits im Alter von 62 Jahren in den Ruhestand eintritt. ${ }^{180}$ Die Vorzeitigkeitsabschläge der Rentenzahlungen werden in der GRV entsprechend der gesetzlichen Regelungen ${ }^{181}$ bestimmt, und in einer Bürgerrente modelliert, weil der Biedenkopf-Miegelsche Entwurf einen vorzeitigen Rentenbezug nicht vorsieht. Hierzu werden die $P E_{i, K}^{B R}$ versicherungsmathematisch fair an die verlängerte Laufzeit der Rentenzahlung angepasst. ${ }^{182}$

Die Ergebnisse sind in Abbildung 18 illustriert. Hierbei werden die Salden in einer Bürgerente (ALL/65/ALL18) für Leistungen in Höhe von 50 v.H. und 65 v.H. sowie für die Situation in der gesetzlichen Rentenversicherung angegeben. Die Darstellung erfolgt exemplarisch für das obere Wirtschaftsszenario und ist im Geldwert von 1999 (umgerechnet auf Euro) angegeben.

Die betrachteten Kohorten sind Nettoempfänger von Transfers, d.h. die realen Werte der Auszahlungen übersteigen die der Einzahlungen.

Für die gesetzliche Rentenversicherung gilt dabei Folgendes: Der Verlauf der Saldenkurven ist in der gesetzlichen Rentenversicherung regressiv. Während sich beispielsweise für einen Standardrentner der Kohorte 1940 ein Saldo in Höhe von $€ 87776$ ergibt, beträgt der Saldo für einen Standardrentner, der im Jahr 1970 geboren worden ist, nur $€ 34053$. Begründet ist dies offenkundig in den Einwirkungen der demographischen Veränderungen, die einen Anstieg des

180 Die Regelaltersgrenze wird mit dem 65. Lebensjahr erreicht (Vgl. § 35 SGB VI). Das durchschnittliche Rentenzugangsalter für Regelaltersrenten beträgt 62,6 Jahre bei Frauen bzw. 62,4 Jahre bei Männern (aBL, Stand 1998, Deutscher Bundestag (1999a, S. 5f.). Hiervon zu trennen ist das durchschnittliche Zugangsalter für Versichertenrenten, das sind Alters- und Erwerbsminderungsrenten. Dies betrug im Jahr 1998 für Frauen in den aBL (nBL) 60,7 (58,2) bzw. für Männern 59,7 (59,9) Jahre (Deutscher Bundestag (1999b, S. 96).

181 Die Zugangsfaktoren $Z F_{s}$ bei vorzeitigem Rentenbezug werden nach den Grundsätzen des $\S 77(2)$ Nr. 2a SGB VI bestimmt, d.h. die gesetzlichen Rentenansprüche werden pro Monat eines Rentenbezugs vor dem vollendeten 65 . Lebensjahr um 0,3 v.H. reduziert.

182 Als Kalkulationszinsfuß wird hierbei der unterstellte langfristige Kapitalmarktzins in Höhe von 4 v.H. verwendet. 
Beitragssatzes und zugleich ein nur mäßiges Wachstum des Rentenwertes erwarten lassen.

\section{Ergebnisse der Referenzszenarien}

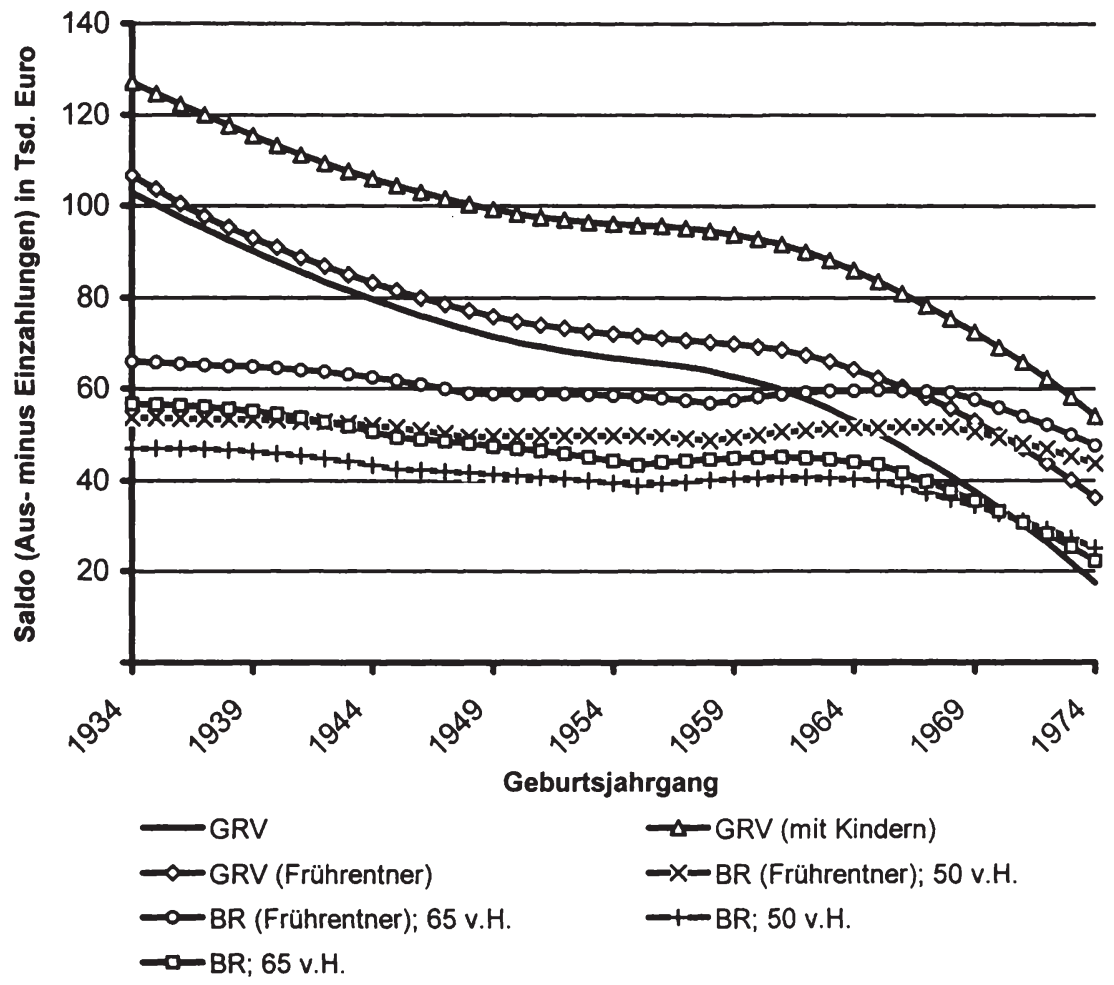

Abbildung 18: Umverteilungen in den Rentensystemen

Zudem zeigt sich, dass die Kindererziehung in der GRV positiv auf die Höhe des Saldos wirkt. Da die Kindererziehungszeiten mit Entgeltpunkten bewertet werden, obwohl diesen keine monetäre Leistung gegenübersteht, sind die Salden im Vergleich zu einem Standardrentner nach oben verschoben.

Wird der Zugangsfaktor bei einem vorzeitigen Rentenbezug stets um 0,3 v.H. pro Monat für einen Rentenbezug vor dem vollendeten 65. Lebensjahr ver- 
ringert, zeigt sich, dass ein Frührentner in der gesetzlichen Rentenversicherung nur geringe Vorteile gegenüber einem Standardrentner besitzt. Für eine im Jahr 1940 geborenen Person beträgt dieser beispielweise nur $€ 3091$.

In einer Bürgerrente zeigt sich ein anderes Bild. Zwar sind auch hier alle betrachteten Kohorten Nettoempfänger von Transfers, jedoch schmelzen hier die Salden aus Aus- und Einzahlungen nur langsam ab. Beträgt der Saldo eines Standardrentners der Kohorte 1940 für ein Leistungsniveau in Höhe von z.B. 50 v.H. (65 v.H.) rund $€ 45934$ ( $€ 54535$ ), ergeben sich für eine Person der Kohorte 1970 rund $€ 32821$ ( $€ 35544$ ). Aufgrund einer Bindung der Rentenhöhe an das Volkseinkommen partizipieren die Individuen auch vom Wachstum der Kapitaleinkommen. Die resultierenden Wirkungen auf den Saldo sind in einem Szenario wirtschaftlicher Stärke folglich geringer als in der gesetzlichen Rentenversicherung.

Werden die Renten im Falle eines vorzeitigen Rentenbezugs in einer Bürgerrente als Auszahlung aus einem Kapitalstock interpretiert und die Teilzahlungen entsprechend der verlängerten Zahlungsdauer verringert, sind die Salden infolge des Zinseffektes größer als bei einem Standardrentner. ${ }^{183}$

In diesem Fall beinhaltet das System der Bürgerrente Anreize, vorzeitig aus dem Erwerbsleben auszuscheiden. Hierbei sei aber betont, dass der Biedenkopf-Miegelsche Originalentwurf einen vorzeitigen Bezug der Rentenleistung nicht vorsieht.

Zusammenfassend gilt, dass das System der gesetzlichen Rentenversicherung und der Bürgerrente für die hier betrachteten Geburtskohorten eine reale Verzinsung der eingezahlten Beiträge bietet. Klar wird auch, dass die Verzinsung in der gesetzlichen Rentenversicherung stark regressiv ist, je später die Individuen der Referenzszenarien geboren werden, desto geringer ist die zu erwartende Rendite. Dies bedeutet aber auch, dass der implizite Steueranteil der GRV, d.h. der Teil der Beitragsleistung, der nicht als Versicherungsprämie interpretiert werden kann, sukzessive ansteigt. ${ }^{184}$

In einer Bürgerrente hingegen bewirkt die Bindung der Rentenhöhe an das Volkseinkommen eine Beteiligung der Rentner am Wachstum der Kapitaleinkommen. In einem Szenario wirtschaftlichen Aufschwunges folgt hieraus, dass

\footnotetext{
183 Maßgeblich ist natürlich der angenommene Kalkulationszinsfuß.

$184 \mathrm{Vgl}$. hierzu auch Abschnitt 2.3, S. 32ff.
} 
sich die Salden aus Aus- und Einzahlungen und damit die reale Rendite nur sehr langsam verringern.

\subsubsection{Zusammenfassung}

Ausgehend von Abfolgen beruflicher Tätigkeiten, die für eine bestimmte Geburtskohorte charakteristisch sind, kann mit einem Modell zur Quantifizierung individueller Rentenansprüche ein unmittelbarer Einkommensvergleich für Altersrentner und Nichtrentner durchgeführt werden. Während sich die Einkommen der Altersrentner im Wesentlichen aus den Bestimmungen des SGB VI bzw. aus den Zahlungen der Bürgerrente und den Einkommen aus eigenständiger Vorsorge ergeben, erfolgt der Vergleich für Nichtrentner auf der Grundlage der bislang erreichten und der zum Zeitpunkt des Eintritts in den Ruhestand zu erwartenden Rentenansprüche.

Als Ergebnis lässt sich hierbei feststellen, dass die Umstellung des Rentensystems auf eine Bürgerrente mit einer Restrukturierung des Gesamtgefüges der Alterssicherung verbunden ist. In einer Umsetzung des Grundgedankens der Subsidiarität gewähren die obligatorischen Leistungen einer Bürgerrente nur ein sozio-kulturelles Existenzminimum. Die Wahrung des in der Erwerbsphase erreichten Lebensstandards gelingt nur in einem Zusammenwirken aller Sicherungssäulen, d.h. die Bedeutung der betrieblichen und der privaten Vorsorge wird besonders betont. Da die Fähigkeit zur eigenständigen Vorsorge eng mit dem Verlauf der individuellen Erwerbsbiographie verbunden ist, gilt auch in einer Bürgerrente, dass das Gesamteinkommen im Ruhestand maßgeblich durch die individuelle Erwerbsbeteiligung bestimmt wird.

Die Substitution der beitragsäquivalenten Leistungen der bestehenden Systeme durch eine pro Kopf einheitliche Rente ist offenkundig für die Personen vorteilhaft, die in den bestehenden Systemen lediglich geringe oder im Extrem keine Rentenansprüche erreichen können. Am Beispiel der Kohorte 1934 lässt sich dies konkretisieren: Hier zählen alle Personen mit Rentenansprüchen in Höhe von bis zu 50 v.H. der Eckrente bei allen betrachteten Leistungsstufen zu den Gewinnern. Im Hinblick auf die stark geschlechtsspezifische Verteilung der Rentenanwartschaften bedeutet dieses Ergebnis insbesondere eine Verbesserung für die Altersicherung der Frauen. Die Bürgerrente kann folglich als ein Beitrag zur geschlechtlichen Gleichberechtigung betrachtet werden.

Neben den Kleinrentnern können auch weitere Personengruppen von einer Systemveränderung profitieren, da die Beitragsbelastungen für alle aus der Literatur bekannten Leistungsstufen einer Bürgerrente geringer als in der GRV sind. 
Die Individuen verfügen also über eine Möglichkeit zur eigenständigen Vorsorge, ohne dass sich die verfügbaren Einkommen in der Erwerbsphase verringern. In Abhängigkeit von der Höhe der Bürgerrente (50 v.H. bis 65 v.H. des Volkseinkommens pro Kopf) betragen die Differenzen zwischen 9,2 und 5,6 Prozentpunkte. Wird die Möglichkeit zur Vorsorge genutzt, kann belegt werden, dass auch Personen mit hohen Rentenansprüchen Vorteile erfahren können. In der Kohorte 1934 zählen z.B. je nach Leistungsniveau zwischen 34,4 v.H. bis 70,0 v.H. der Personen mit Ansprüchen in Höhe von bis unter 75 v.H. der Eckrente zu den Gewinnern. Übersteigen die Forderungen die Eckrente können noch 11,4 v.H. bis 8,0 v.H. Vorteile gegenüber der GRV erzielen. $\mathrm{Ob}$ ein Individuum mit hohen Ansprüchen zu den Gewinner oder zu den Verlierern zählt, wird maßgeblich durch die Ausgestaltung und das Leistungsniveau einer Bürgerrente und durch den Verlauf der individuellen Erwerbsbiographie bestimmt. Hierbei determiniert die Erwerbsbeteiligung die Sparfähigkeit und das Leistungsniveau in Verbindung mit der Ausgestaltung eines solchen Rentensystems implizit die Höhe der möglichen Sparleistungen. Eine Unterbrechung der Erwerbsbeteiligung ist generell mit einer Unfähigkeit zum Sparen und daher mit einer Reduktion des Gesamteinkommens im Ruhestand verbunden. In Ermangelung kompensatorischer Regelungen, man denke hier beispielsweise an die Bewertung rentenrechtlicher Anrechnungszeiten in der gesetzlichen Rentenversicherung, führt also auch eine Unterbrechung infolge von zum Beispiel einer Ausbildung oder - und das wiegt wesentlich schwerer einer Kindererziehung zu einer Verringerung der Alterseinkommen. Die Bürgerrente beinhaltet folglich negative Anreize an Aus- oder Fortbildungen teilzunehmen bzw. Kinder zu erziehen.

Zudem können im Zuge einer Generationenbilanzierung weitere Anreizstrukturen, die mit den beiden Rentensystemen verbunden sind, aufgezeigt werden. Für die gesetzliche Rentenversicherung gilt dabei Folgendes: Das Prinzip der Teilhabeäquivalenz garantiert ein Minimum an intragenerationalen Umverteilungen. Nur diejenigen, die in der Erwerbsphase ein eigenständiges Einkommen erzielen und hiervon Rentenbeiträge entrichten, verfügen im Alter über Leistungen des obligatorischen Rentensystems. Die allokative Verzerrungen der individuellen Arbeitsangebotsentscheidungen werden hierdurch minimiert. Zudem beinhaltet die besondere Bewertung der Kindererziehungszeiten i.d.R. positive Anreize Kinder zu gebären. Werden die Übergangsregeln außer Acht gelassen, kann überdies nachgewiesen werden, dass die Reformbestrebungen zum Abbau der Anreize für Frühverrentungen wirkungsvoll sind. Infolge einer Reduzierung der Rentenansprüche um 0,3 v.H. je Monat eines vorzeitigen 
Rentenbezugs können hierdurch keine nennenswerten Vorteile erzielt werden. ${ }^{185}$

Die Bürgerrente bedingt hingegen eine Abkehr vom Äquivalenzprinzip und damit Umverteilungen, die die individuellen Arbeitsangebotsentscheidungen verzerren. In einer quantitativen Analyse kann belegt werden, dass - wie leicht nachvollziehbar ist - das Ausmaß der Umverteilung positiv mit dem Leistungsniveau korreliert ist. Darüber hinaus kann gezeigt werden, dass ein geringes Leistungsniveau in Höhe von 50 v.H. Umverteilungen in einem Ausmaß induziert, die dem der gesetzlichen Rentenversicherung entspricht. Da die Redistributionen in einer Bürgerrente jedoch ausschließlich durch eine nicht beitragsäquivalente Rentenzahlung und im Unterschied zur GRV nicht z.B. durch eine gesonderte Bewertung der Ausbildungs- oder Kindererziehungszeiten begründet werden, sind diese Ergebnisse vorsichtig zu interpretieren. Aufgrund der Tatsache, dass eine gesonderte Bewertung von rentenrechtlichen Anrechnungszeiten in einer Bürgerrente nicht erfolgt, ist klar, dass ein solches Rentensystem prinzipiell negative Anreize beinhaltet, an Aus- oder Fortbildungen teilzunehmen oder Kinder zu gebären. Zudem kann gezeigt werden, dass eine Bürgerrente Anreize setzen kann, vorzeitig aus dem Erwerbsleben auszuscheiden. Maßgeblich hierfür ist die konkrete Ausgestaltung der rechtlichen Rahmenbedingungen. ${ }^{186}$

Aus der Analyse intra- und intergenerationalen Umverteilungen folgt zudem ein impliziter Anreiz, der grundsätzlich beide Rentensysteme betrifft. Während die heutigen Rentner in der GRV und in einer Bürgerrente eine reale, kapitalmarktäquivalente Verzinsung der entrichteten Beitragsleistungen erreichen, werden nachfolgende Generationen nur noch geringe oder letztlich negative Renditen erzielen. Hieraus folgt, dass der Anteil der Beitragsleistung, der nicht als Versicherungsprämie interpretiert werden kann und eine implizite Steuer begründet, sukzessive ansteigt. Der implizite Anreiz besteht folglich in Ausweichreaktionen, d.h. in einer Umgehung der Beitragsverpflichtung. Während ein solcher Anreiz einem Grundrentensystem mit lohnproportionalen Beitragsleistungen grundsätzlich anhaftet, kann durch diese Analyse belegt werden, dass auch die GRV in zunehmendem Maße mit solchen Wirkungszusammenhängen konfrontiert wird.

185 Hierbei gilt es zu berücksichtigen, dass die Analyse auf dem Rechtsstand nach Ablauf der in den Anlagen 19-21 SGB VI genannten Übergangsregeln basiert.

186 Es ist klar, dass entsprechende Regelungen durchaus auch in einem System der Bürgerrente implementiert werden können. 


\section{Kostenprojektion und Überführung der Rentensysteme}

Eine Vorausberechnung der Kosten eines Rentenreformkonzeptes ist grundsätzlich mit der Problematik verbunden, eine adäquate Vergleichsgröße angeben zu müssen, die die Entwicklung bei einer Beibehaltung der bestehenden Systeme beschreibt. Als eine Folge der langjährigen politischen und wissenschaftlichen Diskussion ist dies trotz oder gerade wegen der Vielzahl der bislang veröffentlichten Studien, der Unterschiedlichkeit der darin getroffenen Annahmen und der hieraus resultierenden Bandbreite möglicher Ergebnisse ein durchaus schwieriges Unterfangen. ${ }^{187}$ Ohne die Berechnungen hinsichtlich ihrer Aussagefähigkeit oder Treffsicherheit (sofern dies überhaupt möglich ist) gegeneinander werten zu wollen, wird im Hinblick auf eine anzustrebende Übersichtlichkeit der hier vorgelegten Projektionen die Studie der Prognos AG (1998) als Referenzgröße für die Fortentwicklung der bestehenden Systeme angesehen. Wie beispielsweise Sinn, Thum (1999) oder der Sozialbeirat (1998) in einer Vergleichbetrachtung verschiedener Prognosen aufzeigen, bewegen sich die Prognos-Ergebnisse zur voraussichtlichen Beitragslast der gesetzlichen Rentenversicherung am unteren Ende des Spektrums vorhandener Kostenschätzungen. Die Prognos-Studie eignet sich daher insbesondere zur Durchführung einer zurückhaltenden Vergleichsanalyse.

Im Einzelnen werden die SOEP-Daten mit den Prognosen zur allgemeinwirtschaftlichen Situation und zur finanziellen Lage der gesetzlichen Rentenversicherung (GRV) des Prognos-Gutachtens ${ }^{188}$ sowie den Ergebnissen der Bevölkerungsfortschreibung des Statistischen Bundesamtes ${ }^{189}$ verknüpft. Damit ergibt sich die Möglichkeit, die Kosten und die Beiträge eines Rentensystems bis zum Jahr $2040 \mathrm{zu}$ bestimmen und zu vergleichen.

Um die Wirkungen veränderter Rahmenbedingungen $\mathrm{zu}$ erfassen, wird eine Bürgerrente mit einem Leistungsniveau in Höhe von 50 v.H. bis 65 v.H. des Volkseinkommens pro Kopf der Wohnbevölkerung untersucht. Die wesentlichen Annahmen der Hochrechnung sind im folgenden Abschnitt zusammengefasst.

187 Vgl. Prognos (1995, 1998 und 2000a), Wissenschaftlicher Beirat beim Bundesministerium für Wirtschaft (1998), Sozialbeirat (1996 und 1999).

188 Vgl. Prognos (1998).

189 Statistisches Bundesamt (2000). 
5.1 Annahmen zur Projektion: Ökonomische und demographische Entwicklung bis 2040

Prognos (1998) stellt die zukünftige Finanzsituation der bestehenden Systeme, hier insbesondere der GRV, anhand von zwei alternativen Szenarien zur gesamtwirtschaftlichen Entwicklung bis zum Jahr 2040 dar. Hierdurch wird implizit ein Korridor möglicher Entwicklungspfade aufgezeigt. Grundlage der hier vorgestellten Projektion ist der gesetzliche Rahmen des Rentenreformgesetzes $1999 .^{190}$

Die untere Variante ist durch eine suboptimale Nutzung der wirtschaftlichen Wachstumspotentiale infolge von Marktabschottungen (Welthandelsvolumen +3,3 v.H. p.a.) und Verteilungskämpfen mit einem Arbeitskostenanstieg oberhalb des Produktivitätszuwachses gekennzeichnet. Das reale Wachstum des Bruttoinlandsproduktes (BIP) beträgt durchschnittlich 0,8 v.H. pro Jahr. Aufgrund eines rückläufigen Beschäftigungsniveaus verharrt die Arbeitslosigkeit durchweg auf einem hohen Niveau.

Im oberen Szenario halten die Liberalisierungstendenzen der Weltwirtschaft an (Welthandelsvolumen $+4,5$ v.H. p.a.), und die Tarifpolitik orientiert sich am Produktivitätszuwachs (nationale Ebene). Die Marktchancen werden besser genutzt, so dass das BIP real um durchschnittlich 1,6 v.H. pro Jahr wächst. Wenngleich das Beschäftigungsniveau zunimmt, ergeben sich infolge der demographischen Verschiebungen erst ab 2010 Entlastungen auf dem Arbeitsmarkt. Die Arbeitslosenquote sinkt bis 2040 auf 4,7 v.H.

Die Abbildung der Bevölkerung erfolgt auf der Grundlage der Bevölkerungsfortschreibung des Statistischen Bundesamtes, in der zwischen drei Varianten möglicher langfristiger Wanderungssalden, V1 (+100000 p.a.) und V2 $(+200000$ p.a.) sowie einem Kontrollszenario V0 $( \pm 0)$, unterschieden wird. Die Altersverteilung der Zuwanderer folgt den Daten des Jahres 1999. In allen Varianten gilt eine im Untersuchungszeitraum konstante Geburtenhäufigkeit in

190 Es sei angemerkt, dass sich inzwischen Veränderungen in der Gesetzeslage (Vgl. z.B. Deutsche Bundesregierung (1998 und 2001d) ergeben haben. Ein Vergleich auf der Grundlage des Rechtsstandes des RRG'99 erscheint dennoch möglich, weil den Reformen u.a. eine Reduktion des Versorgungsniveaus gemein ist. Aktuelle Reformbestrebungen zielen zudem auf eine Wiedereinführung eines Demographie bzw. eines "Nachhaltigkeitsfaktor[s]“" zum Abschmelzen der Rentenansprüche bei steigender Lebenserwartung. Vgl. hierzu Bundesregierung (2003a). 


\section{Entwicklung ausgewählter demographischer und ökonomischer Größen}

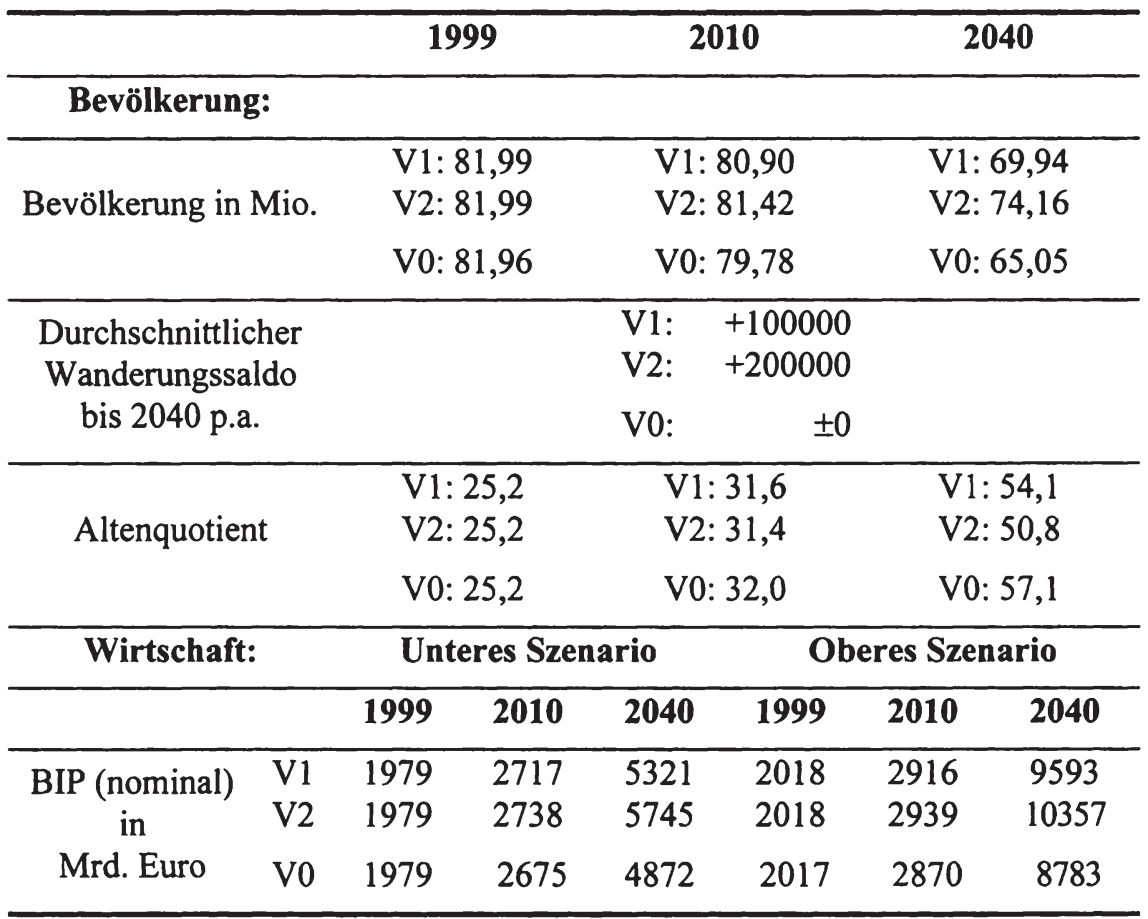

Tabelle 21: Entwicklung ausgewählter demographischer und ökonomischer Größen ${ }^{191}$

Höhe von rund 1,4 Kindern je Frau, und es wird in Anlehnung an den vorherrschenden langfristigen Trend von einer weiteren Zunahme der durchschnittlichen Lebenserwartung ausgegangen. Im Einzelnen erhöht sich diese für ein neugeborenes Mädchen von 80,5 Jahren im Jahr 1998 auf 84,5 im Jahr 2050 bzw. von 74,4 auf 78,1 Jahren für einen Jungen. ${ }^{192}$ Aus diesen Annahmen resultieren signifikante Veränderungen der Bevölkerungsstruktur. Beispielsweise reduziert sich die Gesamtanzahl der Personen von derzeit rund 82,26 Millionen

\footnotetext{
191 In Anlehnung an Prognos (1998) und Statistisches Bundesamt (2000b).

192 Zudem wird angenommen, dass sich die Daten in den neuen und den alten Bundesländern bis zum Jahr 2005 angleichen.
} 
(Stand 2001) auf 64,40 Mio. im Jahr 2050 in der Variante V1 bzw. auf 69,94 Mio. in V2. ${ }^{193}$ Infolge der steigenden Lebenserwartung in Verbindung mit einer geringen Reproduktionsrate ,ergraut" die Bevölkerung zunehmend. In der Variante V1 steigt der Altenquotient ${ }^{194}$ von 25,2 v.H. im Basisjahr 1999 auf 54,1 v.H. im Jahr 2040.

Zur Verknüpfung der demographischen Varianten mit den wirtschaftlichen Szenarien wird generell von einer Exogenität der Fertilitäts- und Mortalitätsraten ausgegangen. Die Wirkungen der unterschiedlichen Wanderungsbewegungen auf das Wirtschaftswachstum werden durch Annahme konstanter proKopf-Beträge für das Bruttoinlandsprodukt, das Volkseinkommen und für die Ausgaben zum Zwecke der Alterssicherung in den bestehenden Systemen abgebildet. ${ }^{195}$ Die wesentlichen Eckpunkte der Szenarien sind der Tabelle $21 \mathrm{zu}-$ sammengestellt.

\subsection{Kostenentwicklung einer eingeführten Bürgerrente - Eine Vor- ausberechnung bis zum Jahr 2040}

Die Vorausberechnung der Kosten erfolgt durch eine unmittelbare Fortführung des Modellansatzes zur „Counterfactual Simulation““. ${ }^{196}$ Die Gesamtkosten $T K^{R}$ eines Rentensystems $R \in\{B R, B S\}$ ergeben sich hiernach aus der Summe der individuellen Bezüge $P E_{t}^{R}$ der Leistungsempfänger $B E_{t}^{R}$ und den Aufwendungen für Rentenäquivalente $K_{t}^{R A E}$, Rehabilitationsmaßnahmen $K_{t}^{\mathrm{Re}}$ sowie für Verwaltungskosten $K_{t}^{V_{w}}$ in einem Jahr $t$.

Für die Vorausberechnung der Gesamtkosten der bestehenden Systeme gilt Folgendes: Die Aufwendungen für Rentenzahlungen $K_{t}^{B S}$ in einem Jahr $t$ ergeben sich aus den Aufwendungen der gesetzliche Rentenversicherung, der Beamtenversorgung und den Zusatzversorgungssystemen im Öffentlichen Dienst. Zur Bestimmung der $K_{t}^{R A E}, K_{t}^{\mathrm{Re}}$ und $K_{t}^{\mathrm{W}_{w}}$ wird ein proportionales Verhältnis

193 Ohne Wanderungen wären es in V0 58,34 Mio.

194 Die Anzahl der Personen in einem Lebensalter von 65 oder mehr Jahren im Verhältnis zur Anzahl der 18- bis 64-Jährigen.

195 Es sei angemerkt, dass es sich hierbei um weitgehende Annahmen handelt. Zur Abbildung der Wirkungsweisen unterschiedlicher Wanderungsströme müssen jedoch Annahmen getroffen werden, die in das Rentenmodell übertragen werden. 
zu den Aufwendungen für Rentenzahlungen in der gesetzlichen Rentenversicherung angenommen.

In einer Bürgerrente ist die Anzahl der Anspruchsberechtigten ein entscheidender Parameter. Zur Projektion der $T K_{t}^{B R}$ gelte Folgendes: Während die Anzahl der Altersrentner direkt aus der jeweiligen Bevölkerungsvariante (V1, V2 oder V0) folgt, ergebe sich die Anzahl der Berechtigten wegen sonstiger Grundlagen aus einer Fortschreibung des aus dem SOEP ermittelten durchschnittlichen Anteils dieser Gruppe an den Personen in einem Lebensalter von 18 bis 64 Jahren. Die Aufwendungen für Rentenäquivalente, Rehabilitationsmaßnahmen und für Verwaltungskosten mögen den Ansätzen in den bestehenden Systemen entsprechen.

Mit diesen Voraussetzungen lassen sich die Kostenpfade der Bürgerrente und der bestehenden Systeme bis zum Jahr 2040 bestimmen. Abbildung 19 illustriert die Ergebnisse am Beispiel der weitergehenden Variante einer Bürgerrente (ALL/65/ALL18) in beiden Wirtschaftsszenarien für die Bevölkerungsvariante V1. Zur Vergleichbarkeit sind die Gesamtkosten eines Rentensystems $T K_{,}^{R}$ als Anteil des Bruttoinlandsproduktes in einem Jahr $t$ angegeben.

Die Darstellung bestätigt das erwartete Bild. Die Aufwendungen für Alterssicherungssysteme werden in beiden Rentensystemen einen größeren Anteil des Bruttoinlandsproduktes erfordern.

Während die Belastungen der bestehenden Systeme (dicke Linien) im oberen Wirtschaftsszenario zunächst um rund 12,5 v.H. des BIP schwanken, beschleunigt sich der Kostenanstieg infolge der demographischen Entwicklung ab dem Jahr 2020 beträchtlich. Eine Fortführung der bestehenden Systeme erfordert beispielsweise im Jahr 2030 Aufwendungen in Höhe von 13,7 v.H. des BIP. Im Jahr 2040 sind es bereits 14,6 v.H. Im Szenario wirtschaftlicher Schwäche hingegen steigen die Kosten aufgrund der annahmegemäß starken Stellung der Arbeitnehmer und der hieraus folgenden hohen Tarifabschlüsse bis zum Jahr 2010 auf 13,4 v.H. und erreichen zum Prognosehorizont im Jahr 2040 rund 16,1 v.H des Bruttoinlandsproduktes.

Ähnliches gilt auch für die Bürgerrente: Auch hier werden die Belastungen deutlich ansteigen. In der Abbildung 19 sind die Kostenpfade einer Bürgerrente (ALL/65/ALL18) für ein Leistungsniveau in Höhe von 50 v.H. (Linien mit Dreiecken) und 65 v.H. (Linien mit Rauten) des Volkseinkommens pro Kopf angegeben. 


\section{Die Entwicklung der Gesamtkosten eines eingeführten Systems einer Bürgerrente}

Oberes Wirtschaftsszenario

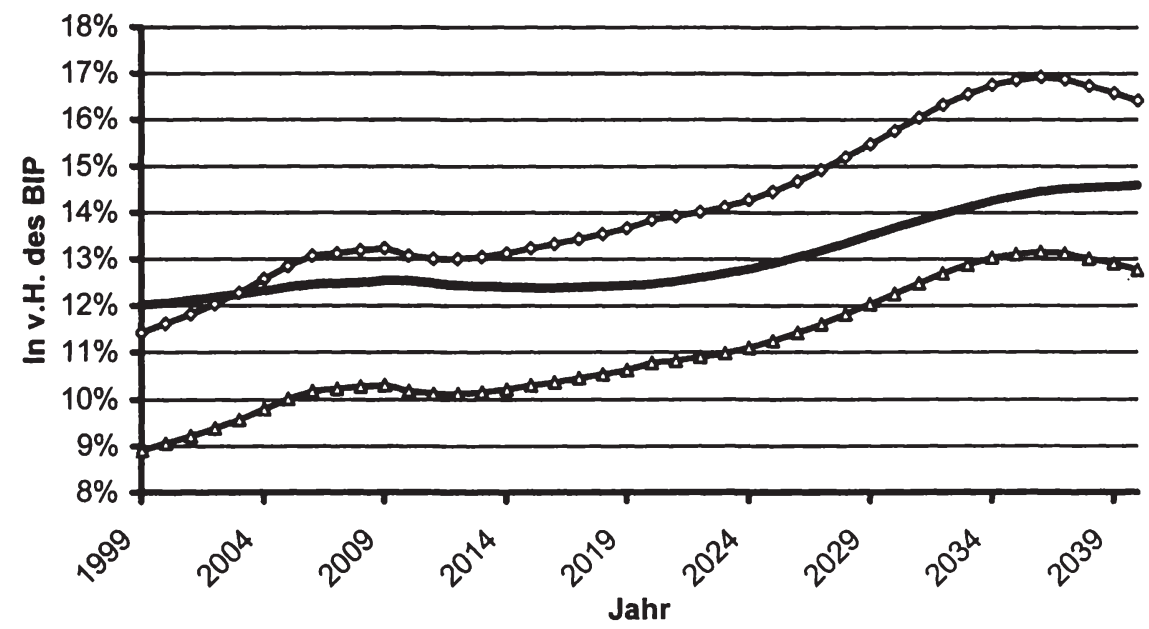

Unteres Wirtschaftsszenario

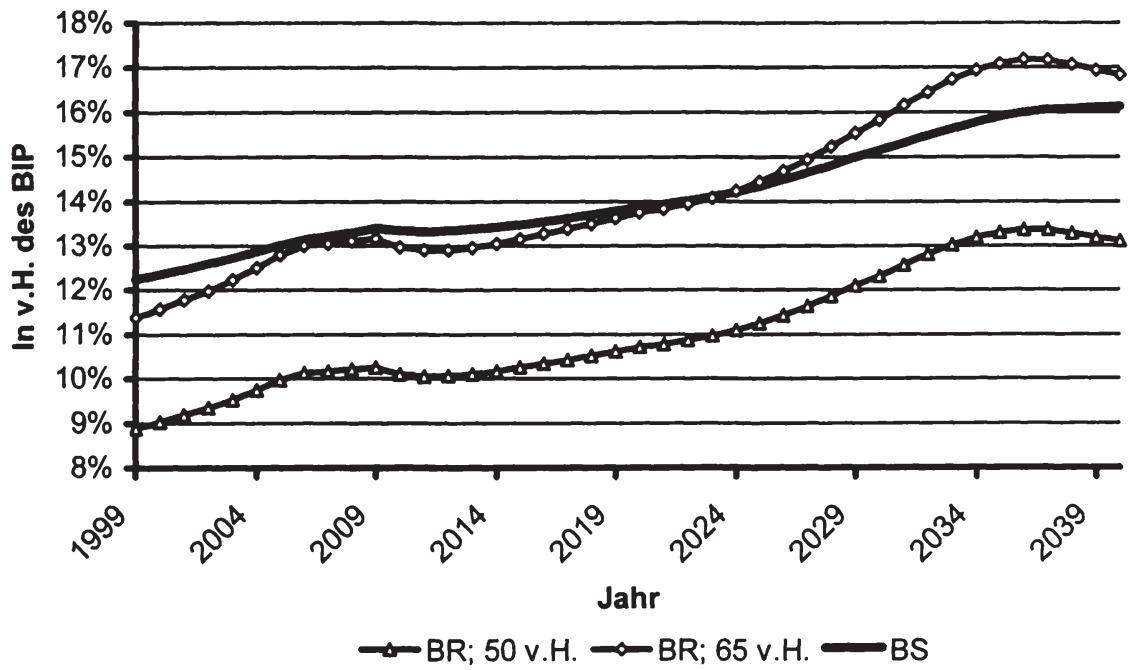

Abbildung 19: Die Entwicklung der Gesamtkosten eines Rentensystems 
Im oberen Wirtschaftsszenario führt ein Leistungsniveau in Höhe von 50 v.H. zunächst zu deutlichen Kostenvorteilen gegenüber den bestehenden Systemen. Im Ausgangsjahr 1999 betragen diese rund 3,1 Prozentpunkte des BIP. Im weiteren Verlauf schmelzen die Vorteile jedoch sukzessive ab und erreichen zum Zeitpunkt der höchsten demographischen Belastung, d.h. im Jahr 2037, ein Niveau in Höhe von rund 1,4 v.H. des BIP. Danach steigen die Vorteile wieder leicht an und betragen zum Prognosehorizont gut 1,8 Prozentpunkte. Im Unterschied hierzu bedingen Leistungen in Höhe 65 v.H. überwiegend höhere Belastungen als in den bestehenden Systemen. Im Extrem betragen diese rund 2,5 v.H. des BIP.

Festzuhalten ist, dass ein geringes Leistungsniveau durchgängig zu Kostenvorteilen gegenüber einer Fortführung der bestehenden Systemen führt. Dieses Ergebnis ist offenkundig vorsichtig zu interpretieren, da die Leistungen eines solches Rentensystems entsprechend gering sind. Zur Analyse der Wirkungen auf die individuellen Einkommen im Ruhestand wird auf Abschnitt 4.2 verwiesen.

Anders im unteren Wirtschaftsszenario: Leistungen in Höhe von 50 v.H. des Volkseinkommens pro Kopf führen hier zu Vorteilen von durchschnittlich 3,0 v.H. des BIP. Und auch Leistungen in Höhe von 65 v.H. bedeuten i.d.R. keine Mehrbelastungen für die Individuen. Die Kostenpfade stimmen für ein solches Leistungsniveau im Wesentlichen mit dem Kostenpfad der bestehenden Systeme überein.

Wird als Maßgröße der Leistungssatz einer Bürgerrente bestimmt, der durchgängig zu Kostenvorteilen gegenüber den bestehenden Systemen führt, zeigt sich, dass im unteren Wirtschaftsszenario Leistungen in Höhe von rund 60 v.H. des Volkseinkommens pro Kopf, das sind in Daten des Jahres 1999 rund $€ 892$ pro Monat, mit Vorteilen verbunden sind. Im wirtschaftlich starken Szenario liegt diese Trennlinie bei nur 55 v.H. des Volkseinkommens pro Kopf oder rund $€ 817$ pro Monat.

Die unterschiedliche Sensitivität der Kostenpfade auf die wirtschaftliche Entwicklung ist in den Berechnungsgrundsätzen der Rentenleistungen begründet. Während die Renten der bestehenden Systeme (hier im Wesentlichen der GRV) an die Entwicklung der Einkommen aus unselbständiger Tätigkeit gekoppelt sind, besteht in einer Bürgerrente eine Bindung an das Gesamteinkommen, z.B. das Volkseinkommen pro Kopf oder das Bruttoinlandsprodukt. 
M.a.W. die wirtschaftliche Entwicklung verändert die Lage des Kostenpfades einer Bürgerrente nur unwesentlich, wohl aber das gesamte Einkommen der Individuen. Entscheidend ist die Anzahl der Berechtigten und somit c.p. die Bevölkerungsstruktur bzw. der Altenquotient. Anders verhält es sich im Falle einer Fortführung der bestehenden Systeme. Hier besitzt neben der Anzahl der Leistungsempfänger auch das unterstellte wirtschaftliche Umfeld einen starken Einfluss auf die Lage des Kostenpfades. Da sich die Lohnentwicklung im oberen Wirtschaftsszenario annahmegemäß am Produktivitätszuwachs orientiert und die Produktion überdies vergleichsweise kapitalintensiv durchgeführt wird, wächst das Volkseinkommen pro Kopf stärker als das Arbeitseinkommen. Im unteren Wirtschaftsszenario hingegen entwickeln sich beide Werte annähernd parallel. Folglich wachsen die Leistungen der bestehenden Systeme in einer Phase wirtschaftlichen Aufschwunges langsamer als die einer Bürgerrente. Der Kostenpfad der bestehenden Systeme verläuft also flacher.

\section{Sensitivität der Kostenpfade auf eine Veränderung der Wanderungssalden}

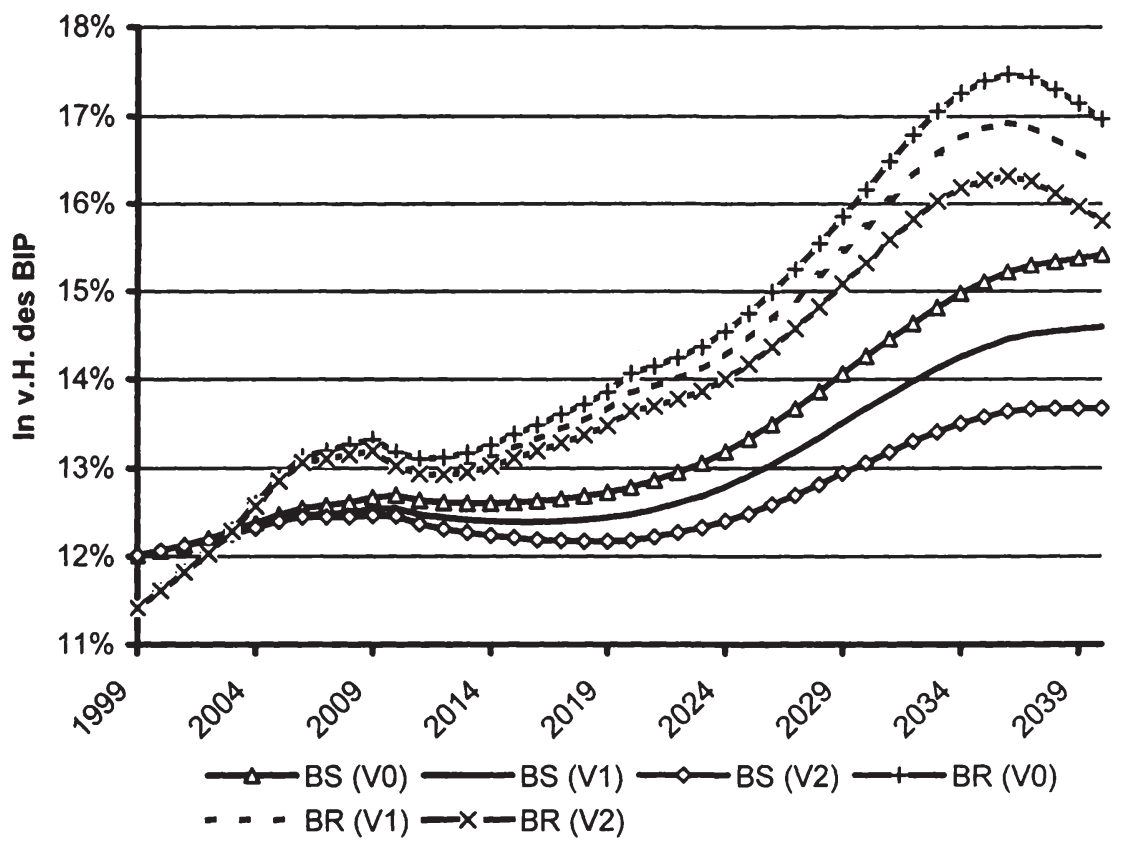

Abbildung 20: Wirkungen unterschiedlicher Zuwanderungssalden 
Ähnliche Ergebnisse ergeben sich auch für die Varianten zur Bevölkerungsentwicklung V2 und V0. Im Falle durchschnittlicher Wanderungsgewinne in Höhe von 200000 Personen pro Jahr (Variante V2) verbleibt der Altenquotient im Extrem mit 51,2 v.H. (Jahr 2037) rund 5,9 v.H. unterhalb des entsprechenden Wertes der Variante V1. Im Kontrollszenario ohne Wanderungsgewinne $\mathrm{V} 0$ beträgt der Altenquotient bis zu 57,4 v.H.

Die resultierenden Kostenwirkungen sind in der Abbildung 20 für das obere Wirtschaftsszenario zusammengestellt. Die Darstellung belegt, dass die Höhe der Zuwanderungssalden einen maßgeblichen Einfluss auf den Kostenpfad der bestehenden Systeme (BS) besitzt. Während sich für das Jahr 2040 in der Variante V1 Kosten in Höhe von 16,3 v.H. des BIP ergeben, sind in einem Szenario mit höheren Wanderungsgewinnen (V2) nur 15,3 v.H. bzw. ohne Wanderungsgewinne (V0) 18,72 v.H. erforderlich.

Da die Leistungen einer Bürgerrente an das Volkseinkommen pro Kopf gebunden sind, entwickeln sich die Hauptkostenfaktoren, nämlich die Bürgerrente wegen Alters und die Bürgerrente wegen sonstiger Anspruchsgrundlagen, im Wesentlichen proportional zum Bruttoinlandsprodukt. Wenngleich veränderte Wanderungssalden die Gesamtkosten einer Bürgerrente maßgeblich bestimmen, bleibt die Lage des Kostenpfades, d.h. das Verhältnis zum BIP, nahezu unverändert. Die Wirkungen veränderter Wanderungsströme sind daher geringer. Für das Jahr 2040 ergeben sind sodann in der Aufwendungen in Höhe von 16,4 v.H. in der Variante V1 und 15,8 v.H. in V2 bzw. 17,0 v.H. in V0.

\subsection{Zusammenfassung}

Die Fiktion einer eingeführten Bürgerrente erlaubt es, eine eingehende Analyse der zukünftigen Entwicklung eines solchen Rentenmodells unabhängig von der spezifischen Ausgestaltung der Übergangsphase durchzuführen. Der gewählte Modellansatz bietet die Möglichkeit, grundsätzliche Wirkungsweisen einer Bürgerrente auf anzunehmende bzw. absehbare Veränderungen der wirtschaftlichen und / oder demographischen Rahmenbedingungen aufzuzeigen.

In einer Projektion bis zum Jahr 2040 zeigt sich, dass eine Bürgerrente erhebliche Kostenvorteile gegenüber einer Fortführung der bestehenden Systeme der Alterssicherung beinhalten kann. Maßgebliche Determinanten der Vorteile sind das Wirtschaftswachstum und offenkundig das gewährte Leistungsniveau einer Bürgerrente. Aufgrund einer Bindung der Leistungen an das Volkseinkommen 
pro Kopf entwickeln sich die Renten im Wesentlichen parallel zum Wirtschaftswachstum. Die Kosten, die eine Volkswirtschaft für Zwecke der Alterssicherung in einer Bürgerrente aufbringen muss, differieren wohl in absoluten Beträgen, sind aber gemessen in Anteilen des Bruttoinlandsproduktes annähernd unabhängig von der wirtschaftlichen Entwicklung.

Anders die Reaktion der bestehenden Systeme: Die Abhängigkeit der Renten vom Wachstum der Einkommen aus unselbständiger Arbeit führt in einer Periode wirtschaftlichen Aufschwungs relativ zum Bruttoinlandsprodukt zu einer unterproportionalen Kostenentwicklung, da die Rentner hier am Wachstum der Kapitaleinkommen nicht partizipieren. Der Kostenpfad der bestehenden Systeme, der den Anteil der Alterssicherungskosten am Bruttoinlandsprodukt misst, verläuft also flacher als der einer Bürgerrente. Geht ein wirtschaftlich schwaches Szenario mit einer starken Stellung der Arbeitnehmervertretungen und entsprechend hohen Tarifabschlüssen einher, ist der Kostenpfad der bestehenden Systeme entsprechend steiler. Im Falle eines wirtschaftlich schwachen Umfeldes ergeben sich also tendenziell größere Kostenvorteile für eine Bürgerrente.

Neben den ökonomischen besitzen offenkundig auch demographische Veränderungen einen maßgeblichen Einfluss auf die zu erwartenden Kosten eines Rentensystems. Wird als demographischer Schock exemplarisch eine Veränderung der Zuwanderungsströme betrachtet, sind die Wirkungen in den bestehenden Systemen der Altersicherung bekannt: Bei Annahme eines konstanten pro-Kopf Betrages des Bruttoinlandsproduktes je Erwerbsfähigen führt eine erhöhte (verringerte) Zuwanderung zu einer geringeren (größeren) Steigung des Kostenpfades.

In einer Bürgerrente zeigt sich ein vergleichbarer Wirkungszusammenhang auf geringerem Niveau. Da sich die Hauptkostenfaktoren einer Bürgerrente, hier die Aufwendungen für Rentenzahlungen, parallel zum Bruttoinlandsprodukt entwickeln, ist die Wirkung veränderter Zuwanderungssalden auf die Kostenpfade geringer. Die bestehenden Systeme reagieren folglich sensitiver auf eine Variation der Zuwanderung.

\subsection{Die Modellierung eines Systemübergangs}

Nach der Vorausberechnung der zu erwartenden Kosten einer eingeführten Bürgerrente gilt es nun, die Umstellung der Systeme zu analysieren. Zentraler Aspekt ist hierbei der Wechsel der Finanzierungsart. Im Einzelnen handelt es 
sich um einen Wechsel vom umlagefinanzierten Konzept („Pay-as-you-go“ oder kurz PAYG) der bestehenden Systeme mit beitragsäquivalenten Renten auf ein Mischsystem, welches sich durch ein umlagefinanziertes Grundrentenkonzept mit pro Kopf einheitlichen Rentenzahlungen und einem ergänzenden privatwirtschaftlich organisierten Kapitaldeckungsverfahren charakterisieren lässt. Die erforderlichen Mittel der Umlagekomponente beider Systeme werden in einer Fortfuihrung des Status quo durch Steuern und lohnabhängigen Beitragsleistungen aufgebracht. Wie aus der Theorie der Alterssicherung bekannt ist, gibt es keinen pareto-optimalen Übergang unter der Voraussetzung eines realistischen Umfeldes - hier insbesondere die Annahme unterschiedlicher Individuen und der Ausschluss von nicht verzerrenden Steuern bzw. Transfers. ${ }^{197}$ Eine Änderung des Alterssicherungssystems bedingt also eine Schlechterstellung einiger Generationen. Ziel des nachfolgenden Abschnittes ist es, die Dauer und das Ausmaß der temporären Mehrbelastung zu quantifizieren, um hieraus möglicherweise Politikempfehlungen ableiten zu können.

\subsubsection{Der Modellaufbau}

\subsubsection{Die Umstellung der gesetzlichen Rentenversicherung}

Die grundlegende Problematik eines Systemwechsels ist in der Rechtsnatur der bereits erworbenen individuellen Rentenansprüche zu sehen, da diesen ein Eigentumscharakter zugesprochen wird. ${ }^{198}$ Innerhalb eines Übergangsszenarios sind diese folglich aufrechtzuerhalten. Die Ansprüche ergeben sich aus dem in Abschnitt 4.2.1 dargestellten Verfahren, welches für Zwecke der Projektion um die von Biedenkopf, Miegel (1997) genannten Übergangsregeln (BM-Regeln) ergänzt wird.

Zur Wahrung des Eigentumscharakters werden im Jahr der Umstellung alle fälligen und nichtfälligen Ansprüche auf das System der Bürgerrente überführt. Wenngleich nur die gezahlten Rentenbeiträge dem verfassungsrechtlichen Schutz unterliegen, wird dieser hier auf die Nettozahlungsgrößen nach Abzug der KVdR / PVdR-Beiträge $\tau_{K V}^{B S}$ bzw. $\tau_{K V}^{B R}$ ausgeweitet. Die zum Zeitpunkt der Umstellung erreichten Nettorenten bleiben somit vollständig erhalten.

\footnotetext{
197 Vgl. Kapitel 2, S. 27ff.

198 Vgl. Fußnote 16, S. 15.
} 
Die Aufstockung der fälligen und nichtfälligen Ansprüche bis zum Regelsatz einer Bürgerrente bzw. die Fortschreibung der nichtfälligen Ansprüche bis zum Erreichen der Regelaltersgrenze erfolgt entsprechend der BM-Regeln. Sei $t^{*}$ das Jahr der Systemumstellung und $T^{*}$ das Jahr, in dem die letzte Person, die Ansprüchen in dem bestehenden System erworben hat, verstirbt. Der monatliche Anspruch $B R_{i, \ell}$ einer Person $i$ auf einen Bezug der Bürgerrente während der Systemumstellung, d.h. innerhalb des Intervalls $\left[t^{*}, T^{*}\right\rfloor$, ergibt sich aus:

$$
\begin{aligned}
& B R_{i, t}=\frac{1-\tau_{K V}^{B S}}{1-\tau_{K V}^{B R}} \cdot P E_{i, i^{\circ}}+\sum_{j=t^{\circ}}^{t} U F_{i, j} \quad \text { Überführungsregel } \\
& \text { mit } \quad U F_{i, j}= \begin{cases}c \cdot B R_{j} & , \text { falls } \frac{B R_{i, j-1}}{B R_{j-1}}<1 \\
\frac{c}{2} \cdot B R_{j} & , \text { falls } \frac{B R_{i, j-1}}{B R_{j-1}} \geq 1 \text { und } a \in\{40, \ldots, 64\} \\
0 & \text {, sonst }\end{cases}
\end{aligned}
$$

Hierbei beschreibt $c$ einen Aufstockungsfaktor der individuellen Rentenansprüche $B R_{i, t}$, die den Regelsatz $B R_{t}$ einer Bürgerrente nicht erreichen. Für die 40- bis unter 65-Jährigen besteht überdies die Möglichkeit, Ansprüche oberhalb des Regelsatzes zu erwirken. Sofern $P E_{i, i^{*}}>B R_{i}$. bzw. $B R_{i, t}>B R_{t}$, werden die Ansprüche dieses Personenkreises in einem jeden Jahr bis zum Erreichen der Regelaltersgrenze, d.h. beispielsweise bis zur Vollendung des 65 . Lebensjahres, um $\frac{c}{2} \cdot B R_{t}$ erhöht. Nach Biedenkopf-Miegel (1997) erfahren hierdurch Personen, die zum Zeitpunkt der Systemumstellung nur über beschränkte beziehungsweise unzureichende Möglichkeiten zur eigenständigen Vorsorge verfügen, eine Kompensation. ${ }^{199}$

199 Im Sinne einer sozial ausgewogeneren Entwicklung der Rentenhöhen über alle Jahrgänge wäre alternativ ein Aufstockungsfaktor $c$ zu benutzen, der sich beispielsweise reziprok zum Lebensalter entwickelt. Ein solcher Faktor könnte z.B. durch $\frac{c}{a^{*}-a^{\prime}}\left(a^{*}\right.$ : Regelaltersgrenze, $a^{\prime}$ Lebensalter zum Zeitpunkt der Systemumstellung) gegeben sein. 
Eine Anwendung der BM-Regel bedeutet aber auch, dass Abhängigkeiten zwischen Individuen, die als Wirtschaftseinheit zu verstehen sind, nicht oder zumindest nicht ausreichend berücksichtigt werden. So kann beispielsweise die Umstellung der fälligen Rentenansprüche eines Rentnerehepaares mit deutlich verschiedenen Anwartschaften (beispielsweise einmal oberhalb und einmal unterhalb des Regelsatzes einer Bürgerrente) dazu führen, dass die hohen Rentenansprüche vollständig erhalten bleiben und zudem die Kleinrente sukzessive auf den Regelsatz angehoben wird. Dies beinhaltet offenkundig einerseits eine erhebliche Besserstellung der Betroffenen, andererseits aber auch eine signifikante Mehrbelastung der Erwerbstätigen. Insofern erscheint es sinnvoll, bei der Umstellung die Gesamtansprüche einer Wirtschaftseinheit, hier z.B. die eines Ehepaares oder einer Haushaltsgemeinschaft, zu erheben und als Grundlage für die Anwendung der Überführungsregeln zu verwenden.

Ein solcher Ansatz wirft natürlich eine Vielzahl von Fragestellungen auf und bedarf zudem einer umfangreichen Datenbasis. Beispielsweise seien hier Fragen der begrifflichen Abgrenzung einer „Wirtschaftseinheit“ - insbesondere im Hinblick auf rechtliche Erwägungen, z.B. den verfassungsrechtlichen Schutz der Ehe - oder des zu berücksichtigen Rentenanspruches - hier Fragen bezüglich einer potenziellen Integration sonstiger Einkünfte, die nicht aus unselbständiger Tätigkeit stammen - genannt. Dies kann hier aus verständlichen Gründen nur erwähnt werden.

Ziel dieser Untersuchung ist es, die durch die Ausgestaltung der Überführungsregeln induzierten Mehrbelastungen begründet zu quantifizieren. Da dies neben den Fragen zur praktischen Umsetzung insbesondere durch eine nur beschränkte Datenverfügbarkeit erschwert wird, sei neben der BM-Regel eine modifizierten Überführungsregel formuliert. Die Aufstockungsbeträge $B R_{i, l}^{\bmod }$ ergeben sich aus:

$$
B R_{i, t}^{\text {mod }}=\frac{1-\tau_{K V}^{B S}}{1-\tau_{K V}^{B R}} P E_{i, i^{+}}+\sum_{j=i^{\dagger}}^{t} U F_{i, j} \quad \text { Überführungsregel }
$$

mit $U F_{i, j}= \begin{cases}c \cdot B R_{j} & , \text { falls } \frac{B R_{i, j-1}^{\bmod }}{B R_{j-1}^{\bmod }}<1 \text { und } a=\{15, \ldots, 64\} \\ 0 & , \text { sonst }\end{cases}$ 
Die modifizierte Überführungsregel (MOD-Regel) betrachtet die Bestandsrentner implizit als Wirtschaftseinheit. Eine Aufstockung der Kleinrenten auf den Regelsatz einer Bürgerrente ist den Personen vorbehalten, die zum Zeitpunkt der Systemumstellung das allgemeine Verrentungsalter, hier das 65. Lebensjahr, noch nicht vollendet haben. Ein Überschießen des Regelsatzes infolge der Aufstockungsbeträge ist ausgeschlossen. Die MOD-Regel gewährleistet also die Aufrechterhaltung der bereits erworbenen Rentenansprüche einer Wirtschaftseinheit und verhindert bzw. verringert zugleich die Möglichkeit zur Wahrnehmung potenzieller „Mitnahmeeffekte“.

\section{Struktur des Modells zur Überführung der Rentenanwartschaften in der gesetzlichen Rentenversicherung}

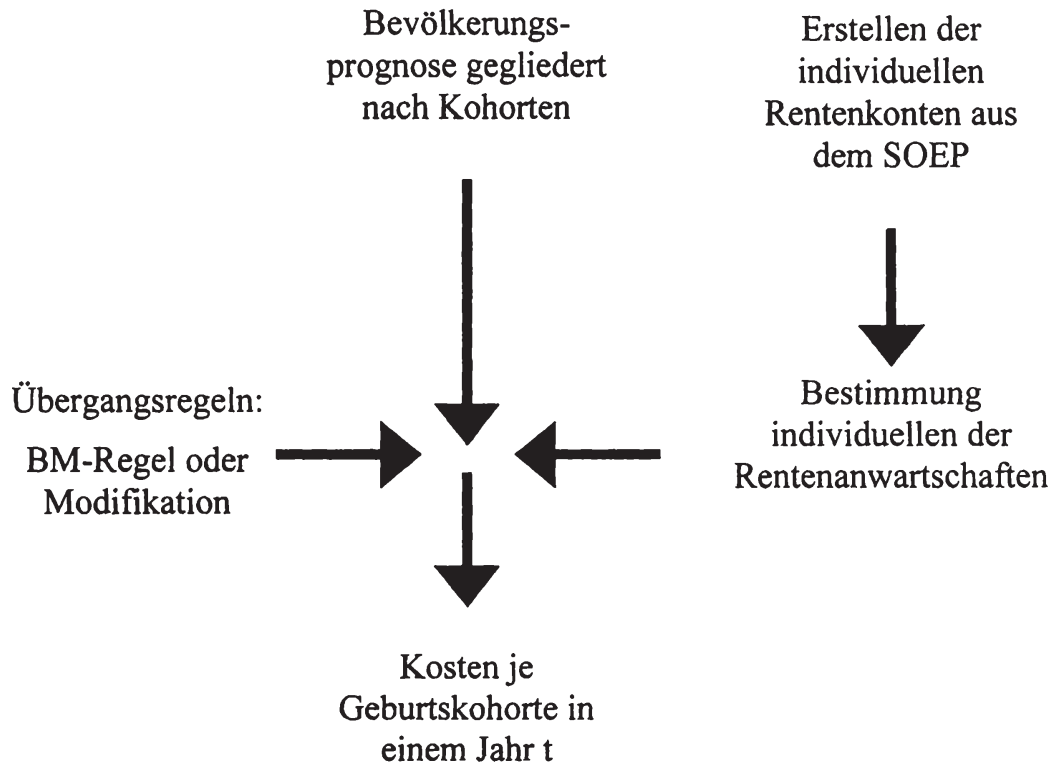

Abbildung 21: Struktur der Übergangsmodellierung für die GRV (Haupteinflussgrößen)

Am Beispiel eines Rentnerpaares wird die veränderte Wirkungsweise deutlich: Die Systemumstellung tangiert die Einkommensverhältnisse des Paares nicht. Die Renteneinkommen werden in gleicher Höhe wie in den bestehenden Sys- 
temen gewährt und entsprechend der wirtschaftlichen Entwicklung dynamisiert. Handelt es sich hingegen um Rentnerpaare oder um alleinstehende Personen oberhalb des Verrentungsalters, d.h. in einem Lebensalter von 65 oder mehr Jahren, mit Rentenansprüchen $B R_{w e, l}^{\bmod }>\mathrm{P}_{\text {we, }}$ (we bezeichnet die betrachtete Wirtschaftseinheit), dann sichert die modifizierte Überführungsregel ausschließlich die zum Zeitpunkt der Systemumstellung bestehenden Rentenansprüche.

Ähnliches gilt auch für die Personen, die zum Zeitpunkt der Umstellung zu den Nichtrentnern zählen. Wird wiederum eine Wirtschaftseinheit mit verschiedenen Ansprüchen (oberhalb und unterhalb des Regelsatzes) betrachtet, dann gewährleistet die MOD-Regel eine Beschränkung bzw. eine Deckelung potenzieller „Mitnahmeeffekte“. Zugleich erfahren Personen in einem Lebensalter von 40 bis 64 Jahren nur begrenzte bzw. keine Kompensationen für entgangene Sparmöglichkeiten.

Kurzum, die Anwendung der beiden alternativen Überführungsregeln ermöglicht es, - ungeachtet der bestehenden Abgrenzungsprobleme - eine Oberbzw. Untergrenze der tatsächlich zu erwartenden Belastungen im Zuge einer Überführung der gesetzlichen Rentenansprüche auf eine Bürgerrente anzugeben. Es resultiert ein Korridor möglicher Lösungswege. Die Grundzüge des Modells zur Umstellung der GRV sind in der Abbildung 21 zusammengefasst.

\subsubsection{Die Überführung der sonstigen Systeme}

Mit Hilfe des vorgestellten Modells gelingt es, die Rentenansprüche der gesetzlich Versicherten zum Zeitpunkt der Umstellung zu bestimmen und auf ein System einer Bürgerrente zu überführen.

Zur Abbildung eines Systemwechsels sind darüber hinaus die Beamtenversorgung (BAV) und ausgewählte sekundäre Alterssicherungssysteme zu berücksichtigen, bei denen vermutet werden kann, dass eine Substitution durch eine Bürgerrente erfolgt. Als ein wichtiges sekundäres System wird hierbei exemplarisch die Zusatzversorgung im öffentlichen Dienst (ZOED) in den Überführungsprozess integriert. Da für diese Systeme geeignete Mikrodaten im SOEP nicht zur Verfügung stehen, gestaltet sich die Überführung schwieriger und ist vermutlich mit größeren Unsicherheiten behaftet. 
Im Zuge der Überführung der $\mathrm{BAV}^{200}$ sind die fälligen und nichtfälligen Versorgungsansprüche $P E_{i, t}^{B V}$ einer Person $i$ in einem Jahr $t$ zu bestimmen. Die den Regelsatz einer Bürgerrente $B R_{t}$ übersteigenden Anteile der fälligen Pensionen sind in einem Ausgleichsbetrag $A B$, zu erfassen. ${ }^{201}$ Im Jahr der Umstellung $t^{*}$ ist $A B_{t}$ offenkundig durch die fälligen Ansprüche der Bestandspensionäre gegeben, die das allgemeine Verrentungsalters $a^{*}$ bereits erreicht haben.

$$
A B_{i}=\sum_{i} P E_{i, t^{*}}^{B V}-B R_{i}: a_{i} \geq a^{*}
$$

Ausgleichsbeträge der

Bestandpensionäre

Die Ausgleichsbeträge aller nachfolgenden Perioden $t>t^{*}$ ergeben sich aus den den Regelsatz übersteigenden Pensionsanteilen der noch lebenden Bestandsund denen der Neupensionäre. Neupensionäre sind Personen mit Versorgungsansprüchen, die in einer Periode $t>t^{*}$ in den Ruhestand eintreten. Wird angenommen, dass sich die durchschnittliche Höhe der Pensionen proportional zu den Einkommen aus unselbständiger Arbeit $Y$, und zur im System erreichten Versicherungszeit $V Z_{i}$ einer Person $i$ ( $V Z$ : maximale Versicherungszeit) entwickelt, dann ergibt sich $A B_{t}$ in einer Periode $t>t^{*}$ aus folgender Fortschreibungs- und Dynamisierungsregel:

$$
A B_{t}=\underbrace{\frac{\mathrm{Y}_{t}}{\mathrm{Y}_{t-1}} \cdot\left(\sum_{i: a_{i} \geq a^{\circ}+t-i} P E_{i, t}^{B V}-B R_{t}\right)}_{\begin{array}{c}
\text { Ausgleichsbeträge der } \\
\text { Bestandspensionäre }
\end{array}}+\underbrace{\frac{\mathrm{Y}_{t}}{\mathrm{Y}_{t-1}} \cdot\left(\sum_{i: a_{i} \geq a^{*}} \frac{V Z_{i}}{V Z} \cdot P E_{i, t}^{B V}-B R_{t}\right)}_{\begin{array}{c}
\text { Ausgleichsbeträge der } \\
\text { Neupensionäre }
\end{array}}
$$

mit

$$
t>t^{*}, V Z_{i}=V Z-\left(t-t^{*}\right)
$$

200 Ohne Versorgungsempfänger nach Kap. I G 131. Aufgrund der geringen Anzahl und des hohen Altersdurchschnittes der Versorgungsempfänger dieser Gruppe, werden diese nicht berücksichtigt. Vgl. hierzu Deutsche Bundesregierung (200lb), S. 38. 
$\mathrm{Da}$ sich die Anzahl der Bestandspensionäre entsprechend der Sterberaten ${ }^{202}$ verringert und sich die Ansprüche der Neupensionäre infolge der verkürzten

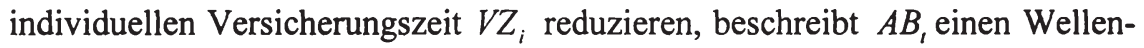
verlauf. Nach einem deutlichen Anstieg folgt ein allmähliches Auslaufen.

Dieses Vorgehen ermöglicht es, die fälligen und nichtfälligen Pensionsansprüche der Versorgungsempfänger des unmittelbaren öffentlichen Dienstes zu bestimmen und in die Modellierung der Systemüberführung zu integrieren.

Da das erklärte Ziel der Zusatzversorgungssysteme des unmittelbaren öffentlichen Dienstes (ZOED) darin besteht, die Rentenansprüche der Arbeitnehmer im öffentlichen Dienst auf die Höhe der Pensionen der Versorgungsempfänger aufzustocken ${ }^{203}$, kann das oben dargestellte Modell zur Berechnung der Ausgleichsbeträge im Wesentlichen auch hier angewendet werden. Im Einzelnen wird dies zur Überführung der Leistungen der Versorgungsanstalt des Bundes und der Länder (VBL), der Arbeitsgemeinschaft kommunale und kirchliche Altersversorgung (AKA) sowie der Bahnversicherungsanstalt Abteilung B (BVA Abt. B) genutzt.

\subsubsection{Die Mehrbelastung im Übergang}

Die numerische Analyse des Systemübergangs erfolgt exemplarisch auf der Grundlage der Daten der 16. Welle (Jahr 1999) des SOEP. Die Ausgangsverteilung der individuellen Rentenansprüche in den bestehenden Systemen ergibt sich aus einer Auswertung der Daten zur Erwerbsbiographie von über 14000 Befragungspersonen, die mit dem im Abschnitt 4.2.1 dargestellten Verfahren in individuellen Rentenkonten zusammengefasst werden. Zur Integration zentraler Leistungsmerkmale der bestehenden Systeme, hier insbesondere die Bewertung der Kindererziehungsleistung in der gesetzlichen Rentenversicherung, sei angenommen, dass stets zwei Kinder zu berücksichtigen sind. ${ }^{204}$ Die Fort-

202 Zur Projektion der Anzahl der Beamten wird der derzeitige Stand (Jahr 2001) proportional zur Anzahl der Erwerbsfähigen fortgeschrieben. Im Vergleich zu Prognos (1998) ergibt sich hierdurch eine leichte Überschätzung der Versorgungsempfänger. Quelle: Statistisches Bundesamt (2000c).

203 Dies bezieht sich vomehmlich auf die Versorgungsrenten der Arbeitnehmer im öffentlichen Dienst. Vgl. hierzu Deutsche Bundesregierung (2001b), S. 42.

204 Zur Betonung der besonderen Bewertung der Kindererziehungszeiten werden durchschnittlich zwei Kinder zur Bestimmung der Ausgangsverteilung der Rentenansprüche angenommen. Vgl. hierzu auch Abbildung 26, S. 169. 
schreibung der Rentenanwartschaften erfolgt durch das im Vorabschnitt dargestellte Überführungsmodell. Die Annahmen zur ökonomischen und demographischen Entwicklung des Abschnittes 5.1 gelten auch hier.

Die Festlegung der Regelaltersgrenzen folgt in beiden Rentensystemen den in den Anlagen 19 bis 21 SGB VI festgelegten Übergangsfristen. Hierdurch wird eine weitgehende Vergleichbarkeit der Kostenverläufe ermöglicht. Die finanziellen Folgen, die hiermit verbunden sind, werden maßgeblich von der individuellen Entscheidung bestimmt, mit Abschlägen vorzeitig aus dem Erwerbsleben auszuscheiden oder bis zur jeweils gültigen Altersgrenze zu arbeiten. Um diese Folgen zu quantifizieren sind Annahmen zu treffen, die in das Modell übertragen werden. Wenngleich die bislang vorliegenden Datenreihen nur wenig Informationen über die zu erwartenden individuellen Verhaltensweisen liefern können, lässt sich ein durchaus preissensitives Verhalten beobachten. Es wird daher ein allmählicher Rückgang der Frühverrentungen angenommen. ${ }^{205}$ Da sich insbesondere bei der Altersrente für Frauen und der Altersrente wegen Arbeitslosigkeit eine größere Wirkung der Rentenabschläge zeigt, wird der Prozentsatz der Personen, der sich für eine Frühverrentung entscheidet, um jährlich 20 v.H. reduziert. Bei Altersrenten wegen langjähriger Versicherung erfolgt eine Anpassung in Höhe von jährlich 5 v.H.

In der Abbildung 22 sind die resultierenden Kostenverläufe beider Überführungsregeln exemplarisch für eine Bürgerrente in Variante (ALL/65/ALL18) bei einer mittleren Leistungsstufe in Höhe von 55 v.H. des Volkseinkommens pro Kopf abgebildet. Im Einzelnen sind der Kostenpfad der BM-Regel (Linien mit Rauten) und der Pfad der MOD-Regel (Linien mit Quadraten) eingezeichnet. Zur Messung der durch den Systemübergang induzierten Mehrbelastung ist in beiden Wirtschaftsszenarien die Fortentwicklung der bestehenden Systeme (dicke Linien) und der Kostenpfad einer eingeführten Bürgerrente (Linien mit Dreiecken) bei entsprechender Leistungsstufe abgebildet. Die Grundlage der hier dargestellten Vorausberechnung ist die Bevölkerungsvariante V1. Zur Veranschaulichung wird die Grafik in drei Abschnitte gegliedert.

Die Darstellung bestätigt das erwartete Bild: Der Systemübergang ist mit zum Teil beträchtlichen Mehrbelastungen verbunden. Dies gilt insbesondere für die ersten Jahre nach einem Systemwechsel, hier dargestellt im ersten Abschnitt der Abbildung 22, die durch das Aufstocken der Kleinrenten auf den Regelsatz 


\section{Mehrbelastungen während des Systemübergangs}

Oberes Wirtschaftsszenario

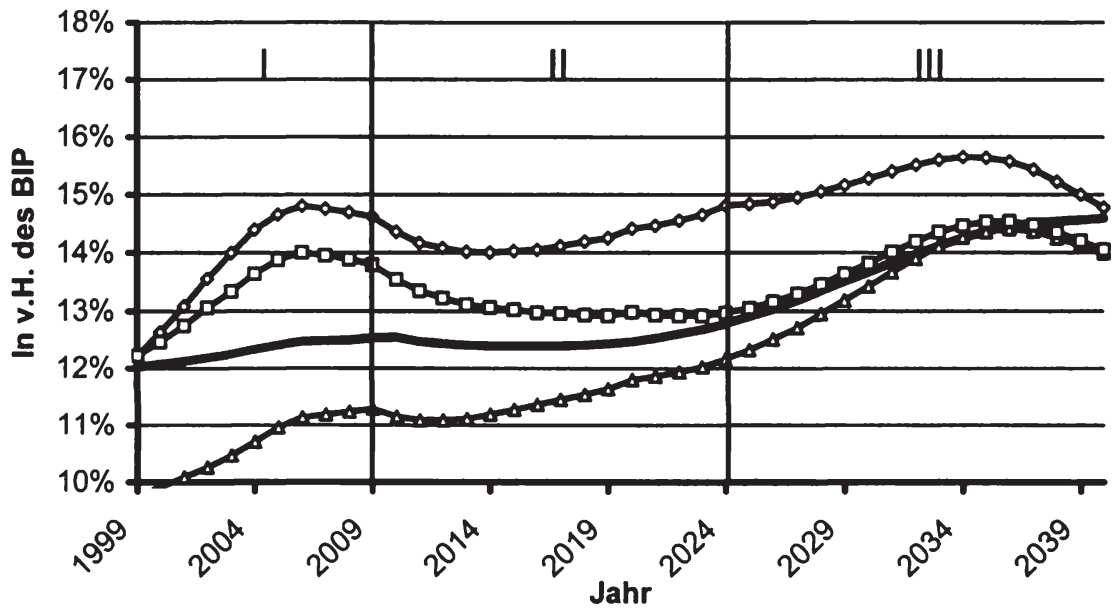

\section{Unteres Wirtschaftsszenario}

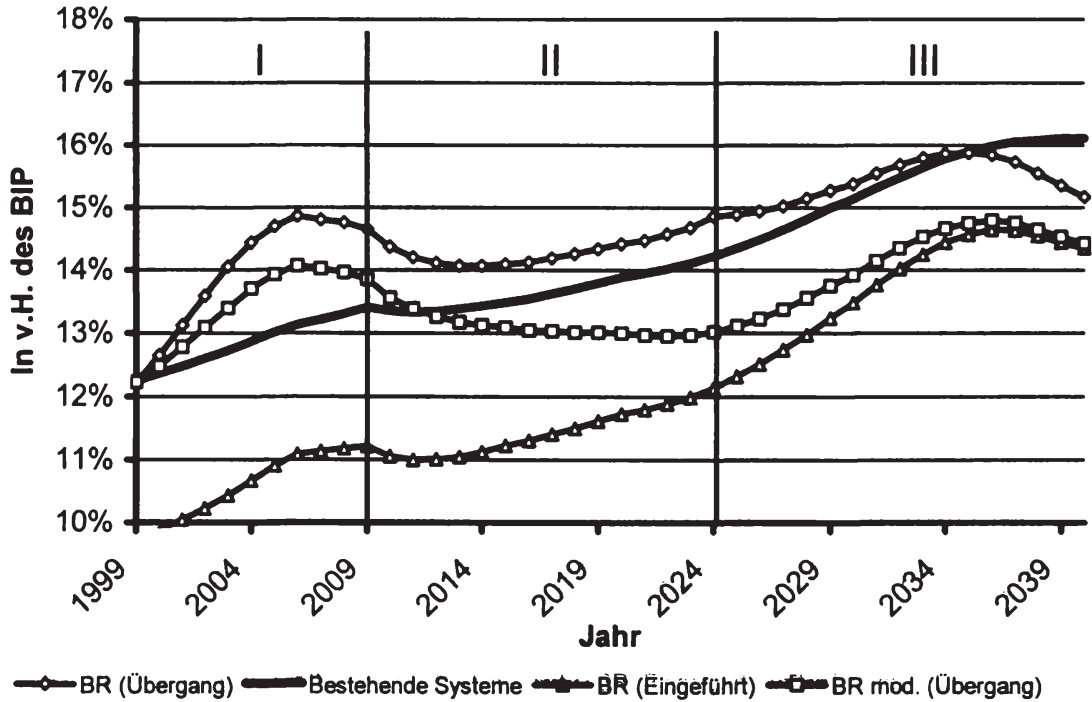

Abbildung 22: Kostenpfade in der Übergangsphase 
einer Bürgerrente geprägt sind. Bei einer Anwendung der BM-Regel steigen die Mehrbelastungen im Szenario wirtschaftlicher Stärke bis zum Jahr 2005 bzw. 2006, d.h. sechs bis sieben Jahre nach der Systemumstellung, auf bis zu 2,3 v.H. des BIP. Im wirtschaftlich schwachen Szenario betragen die Mehrbelastungen aufgrund eines steiler verlaufenden Kostenpfades der bestehenden Systeme im Extrem nur 1,7 v.H. des BIP. Im Falle der MOD-Regel sind die Mehrbelastungen erwartungsgemäß geringer. In der Spitze betragen diese rund 1,6 v.H. bzw. 0,9 v.H. des BIP.

Wenngleich die absoluten Beträge differieren, ist der erste Abschnitt der Systemüberführung, d.h. die ersten zehn Jahre, unabhängig von der unterstellten wirtschaftlichen Entwicklung und der Ausgestaltung der Überführungsregel von bedeutenden Mehrkosten geprägt.

Im zweiten Abschnitt der Überführung, das sind die folgenden 15 Jahre, sind insbesondere die unterschiedlichen Wirkungsweisen der Überführungsregeln von Bedeutung. Während die BM-Regel den Personen in einem Lebensalter von 40 bis unter 65 Jahren aus kompensatorischen Motiven Aufstockungsbeträge auch dann gewährt, wenn die individuellen Rentenansprüche den Regelsatz einer Bürgerrente bereits übersteigen, ist die Aufstockung der Renten in der modifizierten Überführungsregel auf den Regelsatz begrenzt. Hieraus folgt, dass die durchschnittlichen Rentenansprüche der Altersrentner in der MODRegel geringer als in der BM-Regel sind und dass diese sich schneller an den Regelsatz einer Bürgerrente angleichen. ${ }^{206}$ Die resultierenden Kosten einer Bürgerrente sind bei Anwendung der MOD-Regel folglich bedeutend geringer. Der Verlauf der Kostenpfade wird dabei maßgeblich durch die demographischen Einflüsse und durch das sukzessive Zurückführen der Ausgleichsbeträge zur Überführung der Zusatzversorgung im öffentlichen Dienst und der Beamtenversorgung geprägt.

Im wirtschaftlich starken Szenario führt dies dazu, dass die Mehrbelastungen bei der BM-Regel auf einem Niveau in Höhe von rund 1,8 v.H. des BIP verbleiben, während die Kosten bei der MOD-Regel sukzessive abschmelzen und am Ende des zweiten Abschnittes, d.h. rund 25 Jahren nach der Systemumstellung, das Niveau der bestehenden Systeme erreichen. Im Szenario geringen wirtschaftlichen Wachstums führt der steiler verlaufende Kostenpfad der bestehenden Systeme schneller zu einer Kostenparität. Für die MOD-Regel wird diese bereits nach gut zwölf Jahren erreicht.

206 Eine Übersicht über die Entwicklung der durchschnittlichen Rentenansprüche der Altersrentner in der Überführungsphase findet sich in Abbildung 28, S. 170. 
Im dritten Abschnitt, d.h. 25 oder mehr Jahre nach einer Systemumstellung, endet der Prozess der Überführung. In der BM-Regel können Personen, die im dritten Abschnitt in den Ruhestand eintreten, ausschließlich über Rentenansprüche in Höhe des Regelsatzes einer Bürgerrente verfügen. Hierdurch reduzieren sich die durchschnittlichen Rentenansprüche der Altersrentner, und die Kostenpfade nähern sich schrittweise dem Pfad einer eingeführten Bürgerrente an. Im oberen Wirtschaftsszenario führt dies kurz nach dem Jahr 2040 zu einer Kostenparität. Bei Anwendung der modifizierten Übergangsregel stimmen die Kostenverläufe einer Bürgerrente und der bestehenden Systeme zunächst im Wesentlichen überein. Erst nach dem Jahr 2036, d.h. kurz nach dem Überschreiten der demographischen Hauptlast, ergeben sich hier signifikante Kostenvorteile.

In einem wirtschaftlich schwachen Umfeld kann die Kostenparität aus den bereits genannten Gründen schneller erreicht werden. Die BM-Regel führt hier ab dem Jahr 2035 zu Kostenvorteilen, und die MOD-Regel, die innerhalb des gesamten dritten Abschnittes Vorteile beinhaltet, nähert sich allmählich dem Kostenverlauf einer eingeführten Bürgerrente an.

Die Berechnungen zeigen, dass die durch einen Systemwechsel induzierten Mehrbelastungen vor allem im ersten Abschnitt, d.h. in der ersten Dekade nach der Umstellung, zu finden sind. Dies wird offenkundig durch die Aufstockung der Kleinrenten auf den Regelsatz der Bürgerrente bedingt. Das Ausmaß der Mehrbelastungen ist dabei in dem Korridor zwischen der Biedenkopf-Miegelschen- und der modifizierten Überführungsregel als Ober- beziehungsweise als Untergrenze zu erwarten.

\subsection{Zusammenfassung}

Der verfassungsrechtliche Schutz der Rentenanwartschaften gemäß Art. 14 GG gewährt den Rentnern und den Personen, die bereits Ansprüche auf Leistungen der bestehenden Systemen erwirkt haben, einen weitreichenden Bestandsschutz. Die Umstellung der Rentensysteme auf eine Bürgerrente ist daher ein langfristiges Problem, welches buchstäblich erst mit dem Tode des letzten Individuums mit einem Anspruch auf Leistungen dieser Alterssicherungssysteme einen Abschluss findet.

Dennoch kann in einer Projektion der Kosten einer Systemüberführung gezeigt werden, dass die Phase der Mehrbelastungen bedeutend kürzer ist. In Abhän- 
gigkeit vom Wirtschaftswachstum und der Ausgestaltung einer konkreten Überführungsregel können sich bei einem mittleren Leistungsniveau bereits nach rund einer Dekade Vorteile gegenüber einer Fortführung der bestehenden Systemen ergeben. Während die ökonomische Entwicklung infolge der unterschiedlichen Berechnungsgrundlagen der Rentensysteme dazu führt, dass ein geringes Wirtschaftswachstum die Mehrbelastung einer Systemüberführung reduziert, bestimmt die Ausgestaltung der Überführungsregel die Zeitdauer, innerhalb der sich die durchschnittlichen Rentenansprüche der Altersrentner an den Regelsatz einer Bürgerrente anpassen.

Die Formulierung der Überführungsregel besitzt folglich einen entscheidenden Einfluss auf die Höhe und die Dauer der Mehrbelastung eines Systemübergangs. Wird beispielsweise ein Szenario wirtschaftlichen Aufschwunges angenommen, schwanken die Höhen der Mehrbelastungen in Abhängigkeit von der konkreten Überführungsregel zwischen maximal 1,6 v.H. und 2,3 v.H. des Bruttoinlandsproduktes und die Dauer der Mehrbelastung zwischen 25 und gut 40 Jahren. In einem Szenario geringen wirtschaftlicher Stärke beträgt die Höhe der Mehrbelastungen im Extrem zwischen 0,9 v.H. und 1,7 v.H. und die Dauer zwischen zwölf und 35 Jahren.

An diesen Daten wird deutlich, dass die Aufstellung einer solchen Regel auch eine Frage einer intergenerationalen Ausgewogenheit ist. Stellt eine großzügige Überführungsregel zweifelsohne die Personen besser, die zum Stichtag der Systemumstellung zu den Bestandsrentner zählen, so begründet diese zugleich erhebliche Mehrbelastungen der Erwerbstätigen in den folgenden Jahren.

Andererseits führt die konsequente Ausrichtung einer Überführungsregel auf die Umstellung der individuellen Ansprüche auch zu einer bedeutenden Besserstellung der Kleinrentner. Da hiervon insbesondere Frauen profitieren, berührt die Gestaltung der Überführungsregel auch den Bereich der geschlechtlichen Gleichberechtigung. In die Formulierung einer Überführungsregel sind daher Komponenten individueller Gesichtspunkte aber auch Aspekte einer intergenerationalen, sozialen Ausgewogenheit einzubeziehen. Die Gewichtung dieser Komponenten ist eine Frage eines politischen Abwägungsprozesses. 


\section{Schlussbetrachtung}

In der vorliegenden Arbeit wird ein in Anlehnung an Biedenkopf, Miegel (1997) und Beveridge (1942) konzipiertes Grundrentensystem, die Bürgerrente, untersucht. Zentrale Aspekte sind dabei die Ermittlung der Kosten und der Wirkungen eines solches Rentensystems auf die Einkommenssituation der Altersrentner. Ein weiterer Schwerpunkt ist in der Quantifizierung der durch einen Systemübergang induzierten Mehrbelastungen der Erwerbstätigen zu sehen, wobei insbesondere die Höhe und die zeitliche Ausdehnung dieser Mehrbelastungen von Interesse ist.

Hierzu wird ein grundsätzlich neuer Modellansatz zur Analyse eines Rentensystems formuliert. Dieser zeichnet sich dadurch aus, dass die Kosten eines Rentensystems durch eine Aggregation individueller Rentenansprüche bestimmt werden. Es wird also von der Mikro- auf die Makroebene geschlossen. Als Datengrundlage dient dabei im Wesentlichen das Sozioökonomische Panel des Deutschen Instituts für Wirtschaftsforschung in Berlin.

Hierbei konnten die folgenden Ergebnisse erzielt werden:

1. Aus einer Simulation ergibt sich, dass eine Bürgerrente je nach Ausgestaltung erhebliche Kostenvorteile gegenüber den bestehenden Systemen beinhalten kann. Werden die grundsätzlichen Anspruchsgrundlagen des Status quo auf eine Bürgerrente übertragen, so dass die gesicherten Personenkreise und die versicherten Risiken in beiden Systemen übereinstimmen, betragen die Einsparpotentiale für ein Leistungsniveau in Höhe von 50 v.H. des Volkseinkommens pro Kopf (in Größen des Jahres 2001: $€ 655$ netto) rund 30 v.H. Für Leistungen in Höhe von 65 v.H. (in Größen des Jahres 2001: $€ 852$ netto) ergeben sich Einsparungen in Höhe von rund 10 v.H.

Wird eine Grundrente im Sinne des Biedenkopf-Miegelschen Vorschlags begründet, dann beschränkt sich die Anzahl der Berechtigten auf diejenigen, die alle Anspruchsvoraussetzungen erfüllen. Die Kostenvorteile sind dann aufgrund einer geringeren Anzahl der Empfangsberechtigten jeweils um rund sechs Prozentpunkte größer.

2. Diese Ergebnisse sind vorsichtig zu interpretieren. Während das gegenwärtige System die Aufrechterhaltung des in der Erwerbsphase erreichten Lebensstandards anstrebt, orientieren sich die Leistungen einer Bürgerrente an einer anderen Zielsetzung, nämlich an einem Mindestbedarf. In einer Bürgerrente 
kann die Lebensstandardsicherung nur in einem Zusammenwirken aller Tragsäulen der Alterssicherung, hier insbesondere mit der privaten Vorsorge, erreicht werden. Neben den Kosten sind also auch die Wirkungen auf die individuelle Einkommenssituation eines Altersrentners ein konsekutiver Bestandteil der Analyse eines solches Rentensystems.

3. Die Substitution der im Grundsatz teilhabeäquivalenten Renten der bestehenden Systeme durch eine pro Kopf einheitliche Bürgerrente und ergänzender, kapitalgedeckter Einkommen aus privater Vorsorge bedeutet zunächst Vorteile für Personen, die in den bestehenden Systemen nur geringe oder im Extrem keine Rentenansprüche erreichen können. Da die Verteilung der Rentenansprüche stark geschlechtsspezifisch geprägt ist, bedeutet dies insbesondere eine Verbesserung in der Alterssicherung der Frauen.

4. Darüber hinaus zeigt die exemplarische Auswertung der Geburtskohorte 1934, dass auch Personen mit Rentenansprüchen oberhalb des Regelsatzes einer Bürgerrente Vorteile durch eine Systemumstellung erreichen können. Für ein Leistungsniveau in Höhe von 50 v.H. des Volkseinkommens pro Kopf können beispielsweise 34,4 v.H. der Personen mit Rentenansprüchen zwischen 75 v.H. und 100 v.H. der Eckrente (in Größen des Jahres 2001: $€ 782$ bis $€$ 1043) und 11,4 v.H. der Personen mit Ansprüchen oberhalb der Eckrente Vorteile erfahren. Für ein Leistungsniveau in Höhe von 65 v.H. betragen die Gewinneranteile in den genannten Einkommensklassen 70 v.H. bzw. 8,0 v.H. Eine Leistungssteigerung bedingt also eine Einkommensumverteilung. Während Personen mit mittleren Rentenansprüche (hier bis zur Eckrente) profitieren, reduziert sich der Gewinneranteil in der Gruppe der Personen mit hohen Rentenansprüchen, da die mit einer Leistungssteigerung einhergehende Anhebung des Beitragssatzes hier besonders stark wirkt.

5. Die Koppelung der Leistungen an das Volkseinkommen pro Kopf bedingt, dass die Versicherten am Wachstum der Einkommen aus unselbständiger Tätigkeit und am Wachstum der Kapitaleinkommen partizipieren. In einer Projektion der Kosten eines solchen Rentensystems zeigt sich daher ein klassischer Effekt. Während die Kosten der bestehenden Systeme in einem rezessiven Umfeld i.d.R. schneller als das Bruttoinlandsprodukt wachsen, bewirkt die Bindung einer Bürgerrente an das Wachstum der Kapitaleinkommen einen korrigierenden Effekt. Die Kosten einer Bürgerrente entwickeln sich also annähernd parallel zum Wirtschaftswachstum. 
6. Der verfassungsrechtliche Bestandsschutz der Rentenanwartschaften nach Art. 14 GG bedingt, dass der Übergang auf eine Bürgerrente ein langfristiges Problem ist, welches buchstäblich erst mit dem Tode des letzten Individuums mit Ansprüchen auf Leistungen der bestehenden Systeme einen Abschluss findet. In einer quantitativen Analyse eines Übergangsszenarios kann jedoch gezeigt werden, dass die Phase der Mehrbelastungen in Abhängigkeit vom Wirtschaftswachstum, des Leistungsniveaus und von der Ausgestaltung der Überführungsregel durchaus innerhalb eines oder zweier Jahrzehnte durchführbar ist. Werden die Rentenansprüche entsprechend der Biedenkopf-Miegelschen Vorschläge ausschließlich für einzelne Personen erhoben und in einer Bürgerrente dynamisiert, beträgt die Dauer der Mehrbelastung in einem Szenario geringen wirtschaftlichen Wachstums bei Leistungen in Höhe von beispielsweise 55 v.H. des Volkseinkommens pro Kopf rund 35 Jahre. Werden hingegen die Gesamtansprüche von Haushalten als Grundlage der Dynamisierung angesehen, verringert sich die Dauer der Mehrbelastungen aufgrund der geringeren Anzahl der Renten, die auf den Regelsatz aufzustocken sind, auf rund zwölf Jahre. In einem wirtschaftlich expansiven Szenario ergeben sich knapp über 40 Jahre bzw. 25 Jahre. In die Formulierung der Überführungsregel sind daher dem Grundgedanken einer Bürgerrente folgend individuelle Gesichtspunkte aber auch Aspekte einer intergenerationalen, sozialen Ausgewogenheit zu berücksichtigen.

7. Schließlich wird das Ausmaß der Mehrbelastung durch das Wirtschaftswachstum, die Leistungshöhe einer Bürgerrente und die Ausgestaltung der Überführungsregel bestimmt. In einem rezessiven Wirtschaftsszenario betragen die Mehrbelastungen bei einem Leistungsniveau in Höhe von beispielsweise 55 v.H. des Volkseinkommens pro Kopf im Extrem zwischen 0,9 v.H. und 1,7 v.H. des Bruttoinlandsproduktes. In einem expansiven Szenario sind es maximal 1,6 v.H. bis 2,3 v.H.

8. Die Studie zeigt, dass eine Umstellung auf eine Bürgerrente selbst unter der Prämisse einer Aufrechterhaltung der Eigentumsähnlichkeit der bereits erworbenen Rentenanwartschaften möglich und finanzierbar ist. Im Unterschied zu den aus der Literatur bekannten Berechnungen wird die Realisierungsmöglichkeit auf Mikrodatenbasis empirisch begründet. $\mathrm{Ob}$ eine solche Grundrente eingefürt wird, bleibt allerdings vor allem eine Frage eines politischen Abwägungsprozesses. 
Karsten J. Adamski - 978-3-631-75003-2

Downloaded from PubFactory at 01/11/2019 09:24:00AM

via free access 
7. Anhang

7.1 Tabellen und Abbildungen

\section{Beitragssätze in einer Bürgerrente (Variante SEL/65/SEL55)}

\begin{tabular}{c|c|c|c|c|c|c|c}
\hline & $\mathbf{1 9 9 5}$ & $\mathbf{1 9 9 6}$ & $\mathbf{1 9 9 7}$ & $\mathbf{1 9 9 8}$ & $\mathbf{1 9 9 9}$ & $\mathbf{2 0 0 0}$ & $\mathbf{2 0 0 1}$ \\
\hline $\mathbf{G R V}$ & $18,6 \%$ & $19,2 \%$ & $20,3 \%$ & $20,3 \%$ & $19,5 \%$ & $19,3 \%$ & $19,1 \%$ \\
\hline $\mathbf{5 0 , 0} \%$ & $9,2 \%$ & $9,3 \%$ & $9,4 \%$ & $9,1 \%$ & $8,9 \%$ & $9,2 \%$ & $9,1 \%$ \\
\hline $\mathbf{5 2 , 5} \%$ & $9,7 \%$ & $9,8 \%$ & $9,9 \%$ & $9,7 \%$ & $9,5 \%$ & $9,8 \%$ & $9,7 \%$ \\
\hline $\mathbf{5 5 , 0} \%$ & $10,2 \%$ & $10,3 \%$ & $10,5 \%$ & $10,2 \%$ & $10,0 \%$ & $10,3 \%$ & $10,2 \%$ \\
\hline $\mathbf{5 7 , 5} \%$ & $10,7 \%$ & $10,9 \%$ & $11,0 \%$ & $10,7 \%$ & $10,6 \%$ & $10,9 \%$ & $10,8 \%$ \\
\hline $\mathbf{6 0 , 0} \%$ & $11,3 \%$ & $11,4 \%$ & $11,6 \%$ & $11,3 \%$ & $11,1 \%$ & $11,4 \%$ & $11,4 \%$ \\
\hline $\mathbf{6 5 , 0} \%$ & $12,3 \%$ & $12,5 \%$ & $12,7 \%$ & $12,4 \%$ & $12,2 \%$ & $12,5 \%$ & $12,5 \%$ \\
\hline
\end{tabular}

Tabelle 22: Beitragssätze in der Bürgerrente am Beispiel der Variante SEL/65/SEL55 
Bestimmung der Levenshtein-Distanz am Beispiel der zweier Tätigkeitsprofile aus der Geburtskohorte 1974

\begin{tabular}{|c|c|c|c|c|c|c|c|c|c|c|c|c|c|c|c|c|c|c|c|c|c|c|c|}
\hline & Ziel & & 0 & & 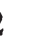 & 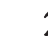 & & & 2 & & & & & & t & & & & 5 & & 4 & & 0 \\
\hline \multirow[t]{2}{*}{ Quell } & & . & & & & & & & & & & & & & & & & & & & & & \\
\hline & $-\quad 0$ & & 0.5 & .5 & 1 & .5 & 1.5 & .5 & 2 & .5 & 2.5 & .5 & 3 & .5 & 3.5 & .5 & 4 & .5 & 4.5 & .5 & 5 & .5 & 5.5 \\
\hline \multirow[t]{2}{*}{10} & $-\quad .5$ & 0 & .5 & 1 & .5 & 1 & & 1 & .5 & 1 & & 1 & & & & & & & & 1 & & & \\
\hline & $-\quad .5$ & .5 & & .5 & 0.5 & .5 & 1 & .5 & 1.5 & .5 & 2 & .5 & 2.5 & .5. & 3 & .5 & 3.5 & .5 & 4 & .5 & 4.5 & .5 & 5 \\
\hline \multirow[t]{2}{*}{4} & $-\quad .5$ & 1 & .5 & 1 & .5 & 1 & & 1 & & 0 & & 1 & & 0 & & 0 & & & & 0 & & & \\
\hline & $-\quad 1$ & .5 & 0.5 & .5 & 1 & .5 & 1.5 & .5 & 2 & .5 & 1.5 & .5 & 2 & .5 & 2.5 & .5 & 3 & .5 & 3.5 & .5 & 4 & .5 & 4.5 \\
\hline \multirow[t]{2}{*}{2} & $-\quad .5$ & 1 & .5 & 0 & .5 & 0 & .5 & 0 & & 1 & & 1 & .5 & 1 & & & & 1 & & & & & \\
\hline & $-\quad 1.5$ & .5 & 1 & .5 & 0.5 & .5 & 1 & .5 & 1.5 & .5 & 2 & .5 & 2.5 & .J & 3 & .5 & 3.5 & .5 & 4 & .5 & 4.5 & .3 & 5 \\
\hline \multirow[t]{2}{*}{2} & $-\quad .5$ & 1 & .5 & 0 & & 0 & .5 & 0 & & 1 & & 1 & & 1 & & 1 & & & & 1 & & & \\
\hline & 2 & .5 & 1.5 & .5 & 1 & .5 & 0.5 & .5 & 1 & .5 & 1.5 & .5 & 2 & .5 & 2.5 & .5 & 3 & .5 & 3.5 & .5 & 4 & .5 & 4.5 \\
\hline \multirow[t]{2}{*}{2} & $-\quad .5$ & 1 & .5 & 0 & & 0 & .5 & 0 & & 1 & .5 & 1 & .5 & 1 & .5 & & & 1 & & & & & \\
\hline & $-\quad 2.5$ & .5 & 2 & .5 & 1.5 & .5 & 1 & .5 & 0.5 & .5 & 1 & .5 & 1.5 & .5 & 2 & .5 & 2.5 & .5 & 3 & .5 & 3.5 & .5 & 4 \\
\hline
\end{tabular}




\begin{tabular}{|c|c|c|c|c|c|c|c|c|c|c|c|c|c|c|c|c|c|c|c|c|c|c|c|}
\hline \multirow{3}{*}{$\frac{\text { Quell }}{4}$} & \multirow{3}{*}{\begin{tabular}{r}
\multicolumn{2}{c}{ Ziel } \\
$-\quad .5$ \\
$-\quad 3$
\end{tabular}} & \multicolumn{2}{|c|}{10} & \multicolumn{2}{|c|}{2} & \multicolumn{2}{|c|}{2} & \multicolumn{2}{|c|}{2} & \multicolumn{2}{|c|}{4} & \multicolumn{2}{|c|}{6} & \multicolumn{2}{|c|}{4} & \multicolumn{2}{|c|}{4} & \multicolumn{2}{|c|}{6} & \multicolumn{2}{|c|}{4} & \multicolumn{2}{|c|}{10} \\
\hline & & 1 & .5 & 1 & & & & 1 & & 0 & & 1 & & & & 0 & & & & & & & \\
\hline & & .5 & 2.5 & .5 & 2 & . & 1.5 & .5 & 1 & .5 & 0.5 & .5 & 1 & .5 & 1.5 & .5 & 2 & .5 & 2.5 & .5 & 3 & .5 & 3.5 \\
\hline \multirow[t]{2}{*}{4} & .5 & & .5 & 1 & & 1 & & 1 & & 0 & & 1 & & 0 & & 0 & & & & 0 & & & \\
\hline & $-\quad 3.5$ & .5 & 3 & .5 & 2.5 & .5 & 2 & .5 & 1.5 & .5 & 1 & .5 & 1.5 & .5 & 1 & .5 & 1.5 & .5 & 2 & .5 & 2.5 & .5 & 3 \\
\hline \multirow[t]{2}{*}{4} & $-\quad .5$ & 1 & .5 & 1 & & 1 & & 1 & & 0 & .5 & 1 & & 0 & & 0 & & 1 & & 0 & & & \\
\hline & 4 & .5 & 3.5 & .5 & 3 & J.J & 2.5 & .5 & 2 & .5 & 1.5 & .5 & 2 & .5 & 1.5 & .0 & 1 & .5 & 1.5 & .5 & 2 & .5 & 2.5 \\
\hline \multirow[t]{2}{*}{4} & $-\quad .5$ & 1 & .5 & 1 & .5 & 1 & & 1 & .5 & 0 & & 1 & & 0 & & 0 & & 1 & & & & & \\
\hline & $-\quad 4.5$ & .5 & 4 & .5 & 3.5 & כ. & 3 & .5 & 2.5 & .5 & 2 & .5 & 2.5 & .5 & 2 & .5 & 1.5 & .5 & 2 & .5 & 1.5 &.$J$ & 2 \\
\hline \multirow[t]{2}{*}{10} & .5 & 0 & .5 & 1 & .5 & 1 & & 1 & .5 & 1 & .5 & 1 & & 1 & & 1 & .5 & 1 & & 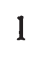 & .5 & & \\
\hline & 5 & .5 & & .5 & 4 & .5 & 3.5 & .5 & 3 & .5 & 2.5 & .5 & 3 & .5 & 2.5 & .5 & 2 & .5 & 2.5 & .5 & 2 & .5 & 2.5 \\
\hline \multirow[t]{2}{*}{10} & $-\quad .5$ & 0 & .5 & 1 & .5 & 1 & .5 & 1 & .5 & 1 & .5 & 1 & .5 & 1 & .5 & 1 & & 1 & & 1 & .5 & & \\
\hline & $\begin{array}{l}-\quad 5.5 \\
\end{array}$ & .5 & & .5 & 4.5 & .5 & 4 & .5 & 3.5 & .5 & 3 & .5 & 3.5 & .5 & 3 & .5 & 2.5 & .5 & 3 & .5 & 2.5 & .5 & 2 \\
\hline
\end{tabular}




\section{Anteil der Gewinner in der Kohorte 1934}

(SEL/65/SEL55)

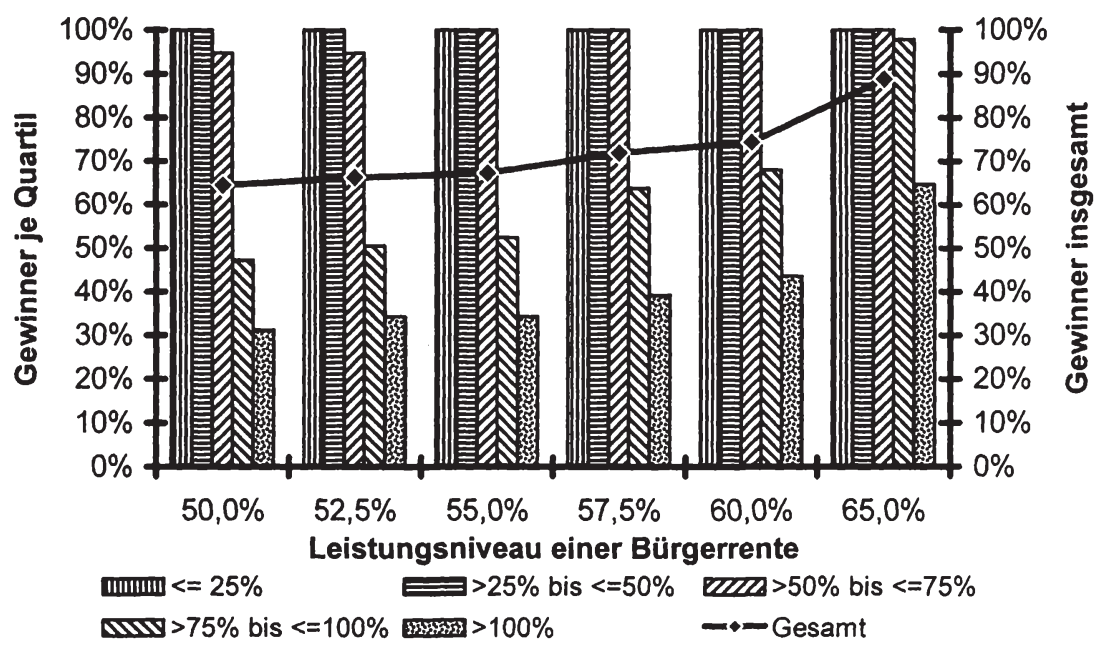

Abbildung 24: Anteil der Gewinner in der Kohorte 1934

\section{Einkommensveränderung in der Kohorte 1934 (SEL/65/SEL55)}

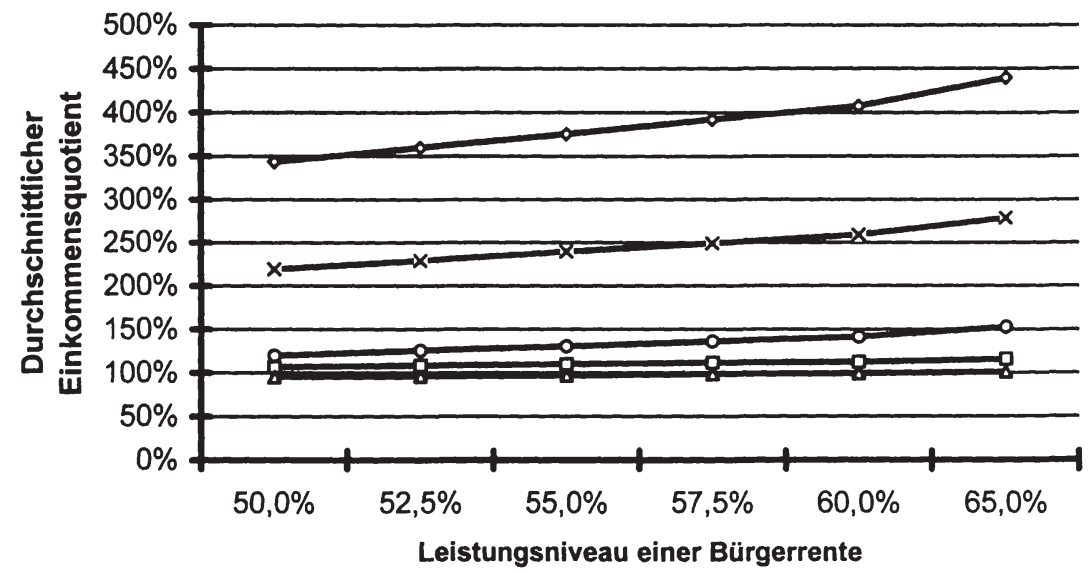

$\longrightarrow<=25 \% \rightarrow-x-25 \%$ bis $<=50 \% \longrightarrow 50 \%$ bis $<=75 \% \rightarrow->75 \%$ bis $<=100 \% \longrightarrow->100 \%$

Abbildung 25: Einkommensveränderung in der Kohorte 1934 


\section{Rentenanwartschaften und Befragungspersonen}

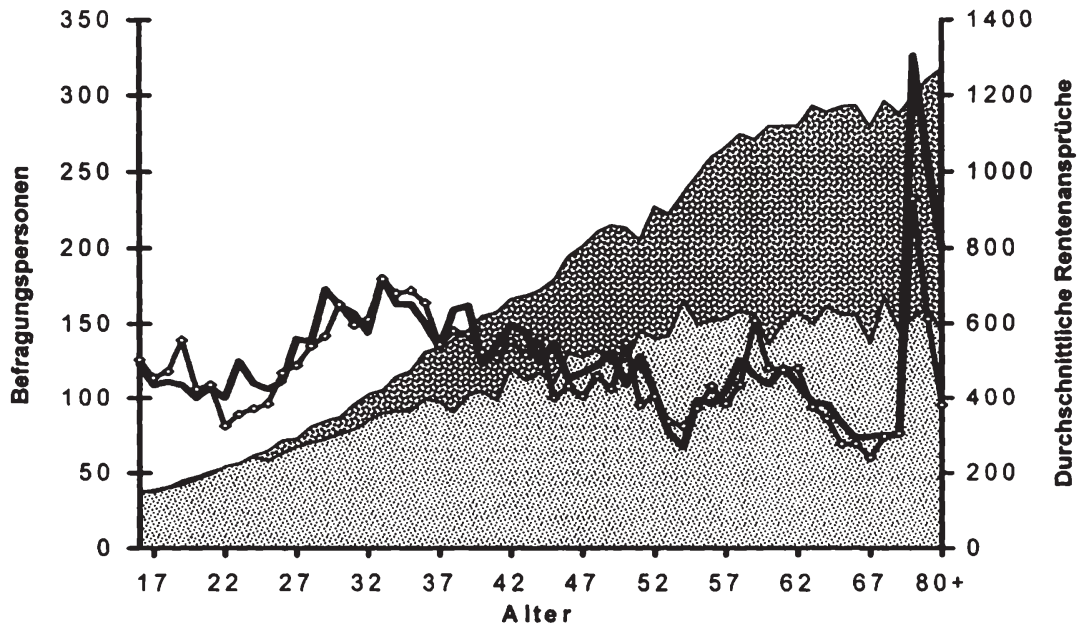

Anspruche Männer Ansprüche Frauen - Befragte Männer Befragte Frauen

Abbildung 26: Rentenanwartschaften und Befragungspersonen

Entwicklung der Rentenansprüche der Neurentner

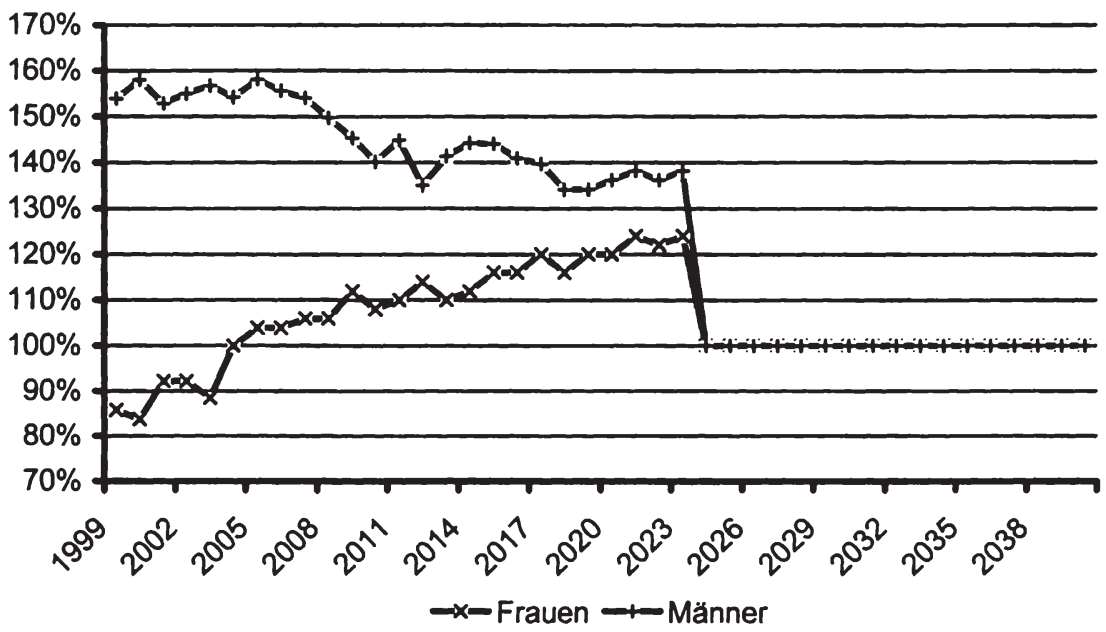

Abbildung 27: Durchschnittlichen Rentenansprüche in der Übergangsphase 


\section{Entwicklung der durchschnittlichen Rentenansprüche der Altersrentner in der Bürgerrente}

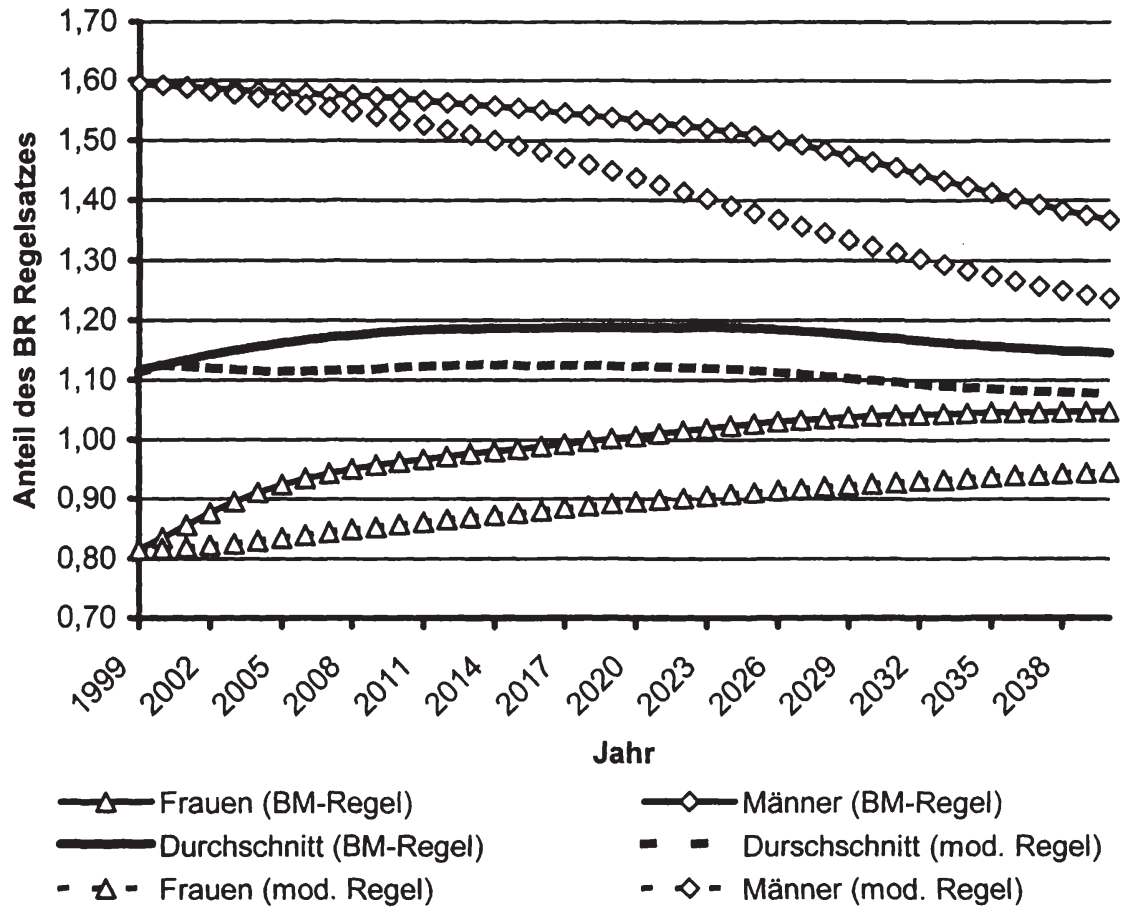

Abbildung 28: Entwicklung der durchschnittlichen Rentenansprüche der Altersrentner in einer Bürgerrente 


\section{2 Überblick über ausgewählte Algorithmen}

Im Folgenden werden die Grundzüge wesentlicher Algorithmen dargestellt. Hierzu wird von den Spezifika einzelner Programmiersprachen abstrahiert und eine allgemeine Darstellung in Form eines „Pseudo Codes“ verwendet.

Sämtliche an die Module übergebenen Variablen werden als global definiert angenommen, d.h. Veränderungen an diesen innerhalb eines Moduls wirken sich auch außerhalb des Moduls aus. Hierzu werden exemplarisch drei Beispiele dargestellt.

Beispiel 1: Bestimmung der Rentenpunkte

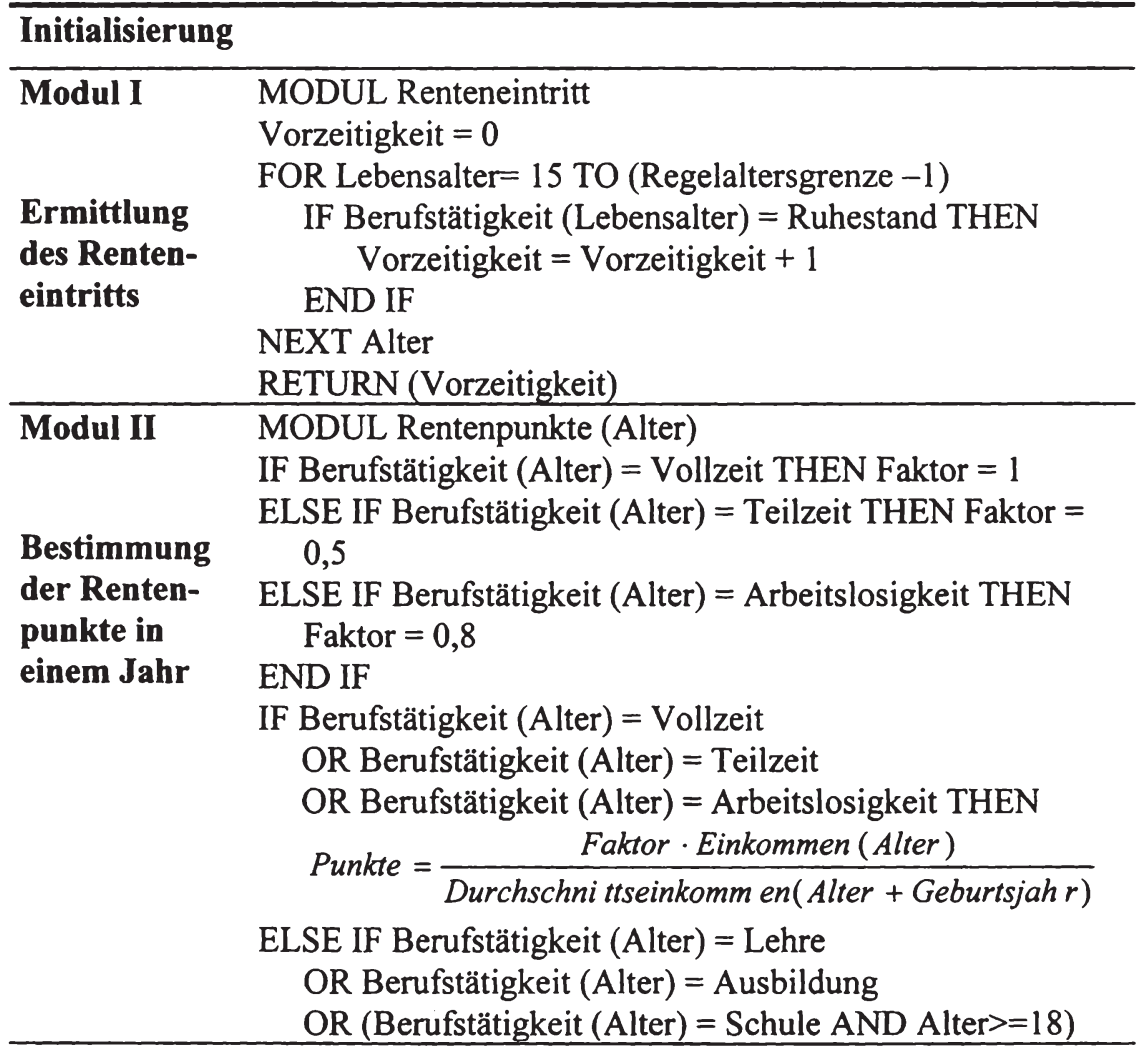


THEN

$$
\text { Punkte }=0,75
$$

ELSE IF Punkte $=0$

END IF

RETURN (Punkte)

\begin{tabular}{|c|c|}
\hline Modul III & MODUL Prognose (Alter) \\
\hline & Summe $=0$ \\
\hline & IF Alter $>=21 \mathrm{THEN}$ \\
\hline Prognose & FOR Zähler $=($ alter-1) TO (alter-5) \\
\hline gem. der & SUMME = SUMME + MODUL Rentenpunkte \\
\hline Renten- & (Zähler) \\
\hline information & NEXT Zähler \\
\hline & Durchschnitt $=0,2 \cdot$ Summe \\
\hline & RETURN (Durchschnitt) \\
\hline Programm & READ Geburtsjahr (Person) aus Datei \\
\hline & READ Akt_Jahr aus Datei \\
\hline & READ Regelaltersgrenze aus Datei \\
\hline & Punkte $=0$ \\
\hline & FOR Alter $=15$ TO $($ Regelaltersgrenze -1$)$ \\
\hline & READ Berufstätigkeit (Alter) aus Datei \\
\hline & READ Einkommen (Alter) aus Datei \\
\hline & $\begin{array}{l}\text { READ Durchschnittseinkommen (Alter + Geburtsjahr) } \\
\text { aus Datei }\end{array}$ \\
\hline & Punkte $=$ Punkte + MODUL Rentenpunkte (Alter) \\
\hline & NEXT Alter \\
\hline & IF Akt_Jahr - Geburtsjahr (Person) < Regelaltersgrenze \\
\hline & THEN Prognose $=($ Regelaltersgrenze - Alter - MODUL \\
\hline & Renteneintritt) $\cdot$ MODUL Prognose (Alter) \\
\hline & ELSE Prognose $=0$ \\
\hline & END IF \\
\hline & Gesamtpunkte $=$ Punkte + Prognose \\
\hline
\end{tabular}

Beispiel 2: Einkommensvergleich der Altersrentner

\begin{tabular}{ll}
\hline Initialisierung \\
\hline Modul I & MODUL GRV_Rente (Jahr) \\
& READ Rentenwert (Jahr) aus Datei \\
Gesetzliche & Zugangsfaktor $=1$ - MODUL Renteneintritt $\cdot 0,036$ \\
Rente & IF Zugangsfaktor $>=0,108$ THEN Zugangsfaktor $=0,108$ \\
\hline
\end{tabular}




\begin{tabular}{|c|c|}
\hline & $\begin{array}{l}\text { GRV_Rente }=0 \\
\text { FOR Alter }=15 \text { TO (Regelaltersgrenze }-1) \\
\quad \text { GRV_Rente }=\text { GRV_Rente }+ \text { MODUL Rentenpunkte } \\
\quad(\text { Alter }) \cdot \text { Rentenwert }(\text { Jahr }) \cdot \text { Zugangsfaktor } \cdot(1-((\text { KVdR } \\
\quad(\text { Jahr })-\text { PVdR (Jahr })) / 2) \\
\text { NEXT Alter } \\
\text { RETURN (GRV Rente })\end{array}$ \\
\hline Modul II & $\begin{array}{l}\text { MODUL BR_Rente (Jahr) } \\
\text { READ Volkseinkommen (Jahr) aus Datei }\end{array}$ \\
\hline Bürgerrente & $\begin{array}{l}\text { BR_Rente }=\text { Volkseinkommen }(\mathrm{Jahr}) \cdot \text { Leistungssatz } \cdot(1- \\
\text { KVdR (Jahr) - PVdR (Jahr) }) / 12 \\
\text { RETURN (BR Rente) }\end{array}$ \\
\hline Modul III & $\begin{array}{l}\text { MODUL Erford_Kapital (GRV_Rente, BR_Rente) } \\
\text { IF GRV_Rente > BR_Rente THEN }\end{array}$ \\
\hline $\begin{array}{l}\text { Erforder- } \\
\text { liches } \\
\text { Gesamt- } \\
\text { kapital }\end{array}$ & $\begin{array}{l}\text { Zahldauer }=\text { Lebenserwartung }- \text { Regelaltersgrenze }+ \\
\text { MODUL Renteneintritt } \\
\text { Erford_Kapital }=\frac{\left(\text { GRV_Re nte }-B R \_ \text {Re nte }\right) \cdot \text { Entsparzins }}{(\text { Entsparzins }+1)^{\text {zahldauer }} \cdot \text { Entsparzins }}\end{array}$ \\
\hline & $\begin{array}{l}\text { END IF } \\
\text { RETURN (Erford Kapital) }\end{array}$ \\
\hline Modul IV & $\begin{array}{l}\text { MODUL Sparguthaben (Person, Jahr, Anteil) } \\
\text { Sparguthaben }=0\end{array}$ \\
\hline $\begin{array}{l}\text { Mögliches } \\
\text { Sparguthaben }\end{array}$ & $\begin{array}{l}\text { FOR Alter = } 15 \text { TO ( Regelaltersgrenze }-1) \\
\text { READ Berufstätigkeit (Person, Alter) aus Datei } \\
\text { READ Einkommen (Alter) aus Datei } \\
\text { IF Beschäftigung (Person, Alter) = Vollzeit THEN } \\
\text { Sparguthaben = Sparguthaben } \cdot(1+\text { Sparzins (Jahr)) } \\
\text { + Einkommen (Alter) Anteil·(GRV_Beitrag (Jahr) - } \\
\text { BR_Beitrag (Jahr)) } \\
\text { ELSE IF Beschäftigung (Person, Alter) } \diamond \text { Ruhestand } \\
\text { THEN } \\
\text { Sparguthaben }=(1+\text { Sparzins }(J a h r)) \cdot \text { Sparguthaben } \\
\text { END IF } \\
\text { NEXT Alter } \\
\text { RETURN (Sparguthaben, Anteil) }\end{array}$ \\
\hline
\end{tabular}




\begin{tabular}{|c|c|}
\hline Modul V & $\begin{array}{l}\text { MODUL DAUER (GRV_Rente, BR_Rente, Person, Jahr, } \\
\text { Anteil) }\end{array}$ \\
\hline \multirow{12}{*}{$\begin{array}{l}\text { Maximale } \\
\text { Bezugsdauer } \\
\text { der Einkom- } \\
\text { men aus dem } \\
\text { möglichen } \\
\text { Sparguthaben }\end{array}$} & IF GRV_Rente > BR_Rente THEN \\
\hline & IF MODUL Sparguthaben (Person, Jahr, Anteil). \\
\hline & $\begin{array}{l}\text { Sparzins }(\text { Jahr })>=\left(G R V \_ \text {Rente }- \text { BR_Rente }\right) \text { THEN } \\
\text { Dauer }=\text { unendlich }\end{array}$ \\
\hline & ELSE \\
\hline & 1 \\
\hline & In $1-$ MODUL Sp arg th.(Pers., Jahr, Anteil)Entspar \\
\hline & $G R V_{-} \operatorname{Re} n t e-B R_{-} \operatorname{Re} n t e$ \\
\hline & $\ln (1+$ Entsparzins $)$ \\
\hline & END IF \\
\hline & END IF \\
\hline & $\begin{array}{c}\text { Dauer }=\text { Lebenserwartung }- \text { Regelaltersgrenze }+ \text { MODUL } \\
\text { Renteneintritt }- \text { Dauer }\end{array}$ \\
\hline & RETURN (Dauer) \\
\hline Modul VI & $\begin{array}{l}\text { MODUL ANTEIL (GRV_Rente, BR_Rente, Person, Jahr, } \\
\text { Anteil) }\end{array}$ \\
\hline Erforder- & Min $=$ Untergrenze \\
\hline licher Anteil & Max $=$ Obergrenze \\
\hline am möglichen & DO REPEAT \\
\hline Sparguthaben & Anteil $=(\operatorname{Min}+\operatorname{Max}) / 2$ \\
\hline & IF MODUL Sparguthaben (Person, Jahr, Anteil) > \\
\hline & MODUL Erford_Kapital (GRV_Rente,BR_Rente) \\
\hline & THEN Max = Anteil \\
\hline & ELSE Min = Anteil \\
\hline & End IF \\
\hline & IF MODUL Sparguthaben (Person, Jahr, Anteil) $=0$ \\
\hline & THEN Anteil $=0$ \\
\hline & EXIT REPEAT \\
\hline & END IF \\
\hline & IF Anteil $\approx$ Obergrenze THEN EXIT REPEAT \\
\hline & UNTIL (1 > MODUL Sparguthaben (Person, Jahr, Anteil) - \\
\hline & MODUL Erford_Kapital (GRV_Rente, BR_Rente) > -1) \\
\hline & RETURN (Anteil) \\
\hline
\end{tabular}




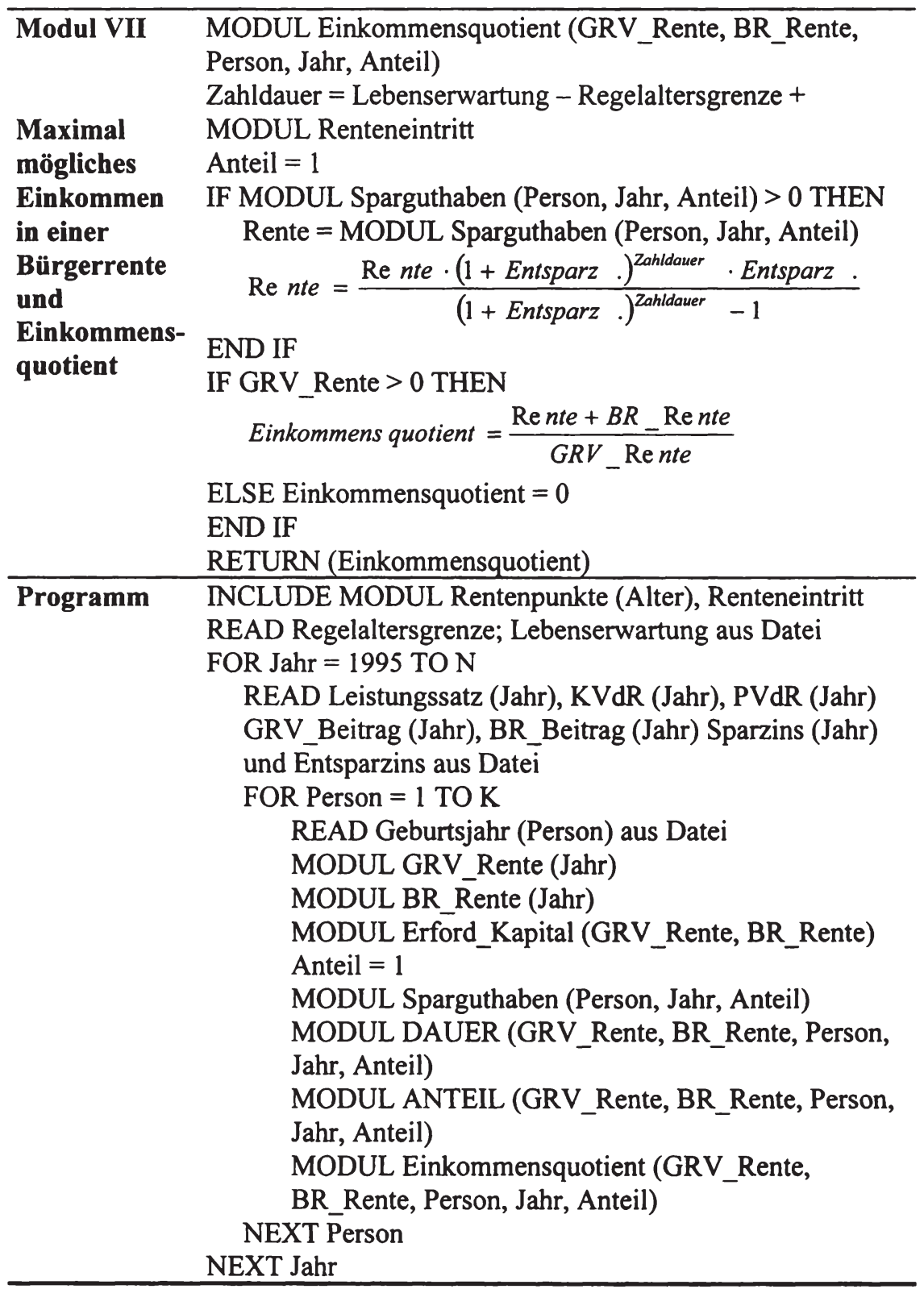


Beispiel 3: Kosten der Überführung

\begin{tabular}{|c|c|}
\hline Initialisierung & \\
\hline Modul I & MODUL Rentenanspruch (Jahrgang, Sex) \\
\hline & FOR Befragungsperson $=$ Erste TO Letzte \\
\hline Ansprüche der & READ Gewicht (Befragungsperson) aus Datei \\
\hline Rentner in & IF Geschlecht (Befragungsperson, Jahrgang) $=$ Sex \\
\hline einem Jahrgang & THEN \\
\hline (Ausgangs- & Punkte $=0$ \\
\hline verteilung) & FOR Alter $=15$ TO $($ Regelaltersgrenze -1$)$ \\
\hline & $\begin{array}{l}\text { Punkte }=\text { Punkte }+ \text { MODUL Rentenpunkte } \\
\text { (Alter) }\end{array}$ \\
\hline & NEXT Alter \\
\hline & Gesamtpunkte $=$ Gesamtpunkte + Punkte . \\
\hline & Gewicht (Befragungsperson, Jahrgang) \\
\hline & END IF \\
\hline & NEXT Befragungsperson \\
\hline & Anspruch $=$ Gesamtpunkte $/$ Gesamtgewicht \\
\hline & GRV_Rente $=$ Anspruch $\cdot$ Rentenwert $($ Akt_Jahr $) \cdot(1-($ \\
\hline & KVdR (Akt_Jahr) + PVdR (Akt_Jahr)) / 2) \\
\hline & BR_Rente $=$ Volkseinkommen (Akt_Jahr) $/ 12$. \\
\hline & Leistungssatz \\
\hline & GRV $=$ Rente \\
\hline & Anspruch $=\frac{\text { BR_Rente) }}{\text { BR_t }}$ \\
\hline & RETURN (Anspruch) \\
\hline Modul II & MODUL Dynamisierung (Jahr, Jahrgang, Anspruch) \\
\hline & Alter $=$ Akt_Jahr - Jahrgang \\
\hline Dynamisierung & IF Alter(Jahrgang) + Jahr $>=100$ THEN Anspruch $=0$ \\
\hline der Rentenan- & ELSE IF Alter(Jahrgang)+Jahr $>=65$ \\
\hline sprüche der & AND Alter(Jahrgang)+Jahr $<100$ THEN \\
\hline GRV-Rentner & $\begin{array}{l}\text { IF Anspruch }<=0,96 \text { THEN Anspruch = Anspruch }+ \\
0,04\end{array}$ \\
\hline & ELSE IF Anspruch $>=1$ THEN Anspruch = Anspruch \\
\hline & ELSE Anspruch $=1$ \\
\hline & END IF \\
\hline & ELSE IF Alter(Jahrgang) $>=40$ \\
\hline & AND Alter(Jahrgang) $<65$ \\
\hline & AND Alter (Jahrgang) $+\mathrm{Jahr}<=65$ THEN \\
\hline & $\begin{array}{l}\text { IF Anspruch }<=0,96 \text { THEN Anspruch }=\text { Anspruch }+ \\
0,04\end{array}$ \\
\hline
\end{tabular}


ELSE IF Anspruch $>=1$ THEN Anspruch = Anspruch $+0,02$

ELSE Anspruch = 1

END IF

ELSE IF Alter(Jahrgang) $<40$

AND Alter (Jahrgang) + Jahr $<=65$ THEN

IF Anspruch $<=0,96$ THEN Anspruch = Anspruch + 0,04

ELSE IF Anspruch >= 1 THEN Anspruch = Anspruch

ELSE Anspruch $=1$

END IF

END IF

RETURN (Anspruch)

Modul III MODUL Ueberführung (Jahr, Jahrgang)

REM Anspruch der Bestandsrentner und -pensionäre

Überführung

FOR Alter $=$ Regelaltersgrenze TO 100

der Versorgungsansprüche

IF Alter $>=$ Regelaltersgrenze + Jahr THEN

Personen $(\mathrm{Jahr})=$ Personen $(\mathrm{Jahr})+$ Personen (Alter)

END IF

NEXT Alter

Berechtigte $(\mathrm{Jahr})=$ Berechtigte $\cdot \frac{\text { Personen }(\mathrm{Jahr})}{\text { Personen }}$

Leistung $($ Jahr $)=$ Leistung $\cdot \frac{\text { Einkommen }(\text { Jahr })}{\text { Einkommen }}$

IF Leistung (Jahr) $>$ MODUL BR_Rente (Jahr) THEN

Anspruch_Bestandsrentner $=$ Leistung $(\mathrm{Jahr})$.

Berechtigte (Jahr) - MODUL BR_Rente (Jahr)

ELSE Anspruch_Bestandsrentner $=0$

END IF

REM Anspruch der Neurentner und -pensionäre

FOR Alter $=$ Regelaltersgrenze TO 100

IF Alter $>=$ Regelaltersgrenze

AND Alter $>=$ Regelaltersgrenze + Jahr THEN

Personen $(\mathrm{Jahr})=$ Personen $(\mathrm{Jahr})+$ Personen

(Alter)

END IF

NEXT Alter 
Berechtigt e $($ Jahr $)=$ Berechtigt e $\cdot \frac{\text { Personen }(\text { Jahr })}{\text { Personen }}$

IF Jahr $<=40$ THEN

$$
\text { Wartezeit }=\frac{40-J a h r}{40}
$$

ELSE Wartezeit $=0$

Leistung $(\mathrm{Jahr})=$ Leistung $\cdot$ Wartezeit $\cdot \frac{\text { Einkommen }(\mathrm{Jahr})}{\text { Einkommen }}$

IF Leistung (Jahr) > MODUL BR_Rente (Jahr) THEN

Anspruch_Neurentner = Leistung $(\mathrm{Jahr})$.

Berechtigte (Jahr) - MODUL BR_Rente (Jahr)

ELSE Anspruch_Neurentner $=0$

END IF

Versorgungsanspruch $=$ Anspruch_Bestandsrentner +

Anspruch Neurentner

RETURN (Versorgungsanspruch)

Modul IV MODUL Beamte_und_ZOED (Jahr, Jahrgang)

Versorgung $=0$

Überführung

der Beamtenversorgung und der ZOED
FOR System $=$ Beamte TO ZOED

READ Anzahl der Personen oberhalb der Regelaltersgrenze im Erhebungsjahr (Personen), Höhe der Einkommen aus unselbständiger Tätigkeit im Erhebungsjahr (Einkommen), Anzahl der Berechtigten (System) im Erhehungsjahr (Berechtigte), Höhe der Leistung (System) im Erhebungsjahr (Leistung) aus Datei Versorgung $=$ Versorgung + MODUL Ueberführung (Jahr, Jahrgang)

NEXT System RETURN (Versorgung)

Modul VI MODUL Ausgleich_Regelaltersgrenze (Jahr) Frühverrentungsart__ $1=$ Langjährig Versichert

Anhebung RegelaltersFrühverrentungsart_2 = Altersrente wegen Arbeitslosigkeit grenze
Frühverrentungsart_3 = Altersrente für Frauen

FOR Alter $=60 \mathrm{TO}($ Regelaltersgrenze -1$)$

FOR Zähler = Frühverrentungsart_1 TO

Frühverrentungsart 3

READ Anteil der Frührentner (Anteil) der Frühverrentungsart_Zähler im Lebensalter 
$=$ Alter zum Zeitpunkt $($ Jahr -1$)$ aus Datei

READ Anzahl der Personen (Personen ) im

Lebensalter $=$ Alter in einem $\mathrm{Jahr}=\mathrm{Jahr}$ aus Datei

IF Zähler = Frühverrentungsart_1 THEN

IF 0,95 - Anteil $(\mathrm{Jahr}-1)>$ Mindestwert

THEN Anteil $(\mathrm{Jahr})=0,95 \cdot$ Anteil $(\mathrm{Jahr}-1)$

ELSE Anteil $=$ Mindestwert

END IF

ELSE IF Zähler = Frühverrentungsart_2

OR Zähler = Frühverrentungsart_3 THEN

END IF

Anteil $(\mathrm{Jahr})=0,80 \cdot$ Anteil $(\mathrm{Jahr}-1)$

Ausgleichskosten $=$ Ausgleichskosten + Personen

NEXT Alter

(Jahr) · Anteil (Jahr)

RETURN (Ausgleichskosten)

Programm INCULDE MODUL BR_Rente (BR_Rente)

INCULDE MODUL Rentenpunkte (Alter)

READ Aktuelles Jahr (Akt_Jahr)

FOR Jahr 1 TO Z

Gesamtanspruch $=0$

READ Leistungssatz (Jahr), KVdR (Jahr), PVdR (Jahr)

FOR Jahrgang $=$ Ältester TO Jüngster

FOR Sex = Frau To Mann

MODUL Rentenanspruch (Jahrgang, Sex)

READ Personenanzahl (Jahr, Jahrgang, Sex)

Anspruch (Sex) $=$ MODUL Dynamisierung

(Jahrgang, Jahr, Anspruch) · Personenanzahl

NEXT Sex

Gesamtanspruch $=$ Gesamtanspruch + Anspruch

(Frau) + Anspruch (Mann)

NEXT Jahrgang

Gesamtkosten $(\mathrm{Jahr})=$ Gesamtanspruch + MODUL

Beamte_und_ZOED (Jahr, Jahrgang) + MODUL

Ausgleich_Regelaltersgrenze (Jahr)

NEXT Jahr 


\subsection{Abkürzungsverzeichnis}

AdL Alterssicherung der Landwirte

AKA Arbeitsgemeinschaft der kommunalen und kirchlichen Altersversorgung

BSHG Bundessozialhilfegesetz

BAV Beamtenversorgung

BIP Bruttoinlandsprodukt

BM-Regel Überführungsregel nach Biedenkopf, Miegel (1997)

BVA Abt. B Bahnversicherungsanstalt Abteilung B

BSV Berufsständische Versorgungssysteme

c.p. certeris paribus

GRV Gesetzliche Rentenversicherung

i.d.R. In der Regel

OMA Optimal Matching Analysis

HBL Hilfe in besonderen Lebenslagen

HLU Hilfe zum Lebensunterhalt

KDV Kapitaldeckungsverfahren

KVdR Krankenversicherung der Rentner

MOD-Regel Modifizierte Überführungsregel

PAYG Pay-as-you-go

PVdR Pflegeversicherung der Rentner

SOEP Sozioökonomisches Panel

UV Umlageverfahren

VBL Versorgungsanstalt des Bundes und der Länder

VDR Verband Deutscher Rentenversicherungsträger

v.H. Vom Hundert

ZOED Zusatzversorgung im öffentlichen Dienst 


\subsection{Symbolverzeichnis}

$\beta_{\lambda}$

$\lambda_{i, t}$

$\varepsilon_{k, K}$

$\Lambda$

$\mu_{k, K}$

$v_{t}$

$\pi_{k, K}$

$\tau^{K V}$

$\tau_{1}^{R}$

$\varpi$

$\Omega$

$a$

$a^{*}$

$A B$,

br,

$B E_{t}^{R}$

$B R_{t}$

$B R_{t, i}$

$B R_{i, I}^{\bmod }$

c
Tätigkeitsartfaktor der Tätigkeit $\lambda$

Tätigkeit $\lambda \in \Lambda$, die eine Person $i$ im Jahr $t$ überwiegend ausübt

Proportionalitätsfaktor zur Proratisierung der Rentenansprüche bei unvollständigen Erwerbbiographien

Menge der möglichen Tätigkeiten

Anteil der möglichen Sparleistung $S_{k, K}^{(a)}$ der zu TC führt

Diskontfaktor im Jahr $t$

Einkommensquotient

Beiträge der Rentner eines Rentensystems $R$ zur Kranken- und Pflegeversicherung der Rentner

Beitragssatz in einem Rentensystem $R$

Eine elementare Operation $\varpi \in \Omega$

Menge der möglichen elementaren Operationen

Lebensalter (in den Grenzen $[0, A]$ )

Verrentungsalter

Ausgleichsbeträge der Versorgungsempfänger

Leistungsniveau einer Bürgerrente in v.H. des Volkseinkommens pro Kopf in einem Jahr $t$

Leistungsbezieher eines Rentensystem $R$ im Jahr $t$

Regelsatz einer Bürgerrente $i$ im Jahr $t$

Ansprüche auf die Bürgerrente einer Person $i$ in der Überführungszeit bei Anwendung der BM-Regel

Ansprüche auf die Bürgerrente einer Person $i$ in der Überführungszeit bei Anwendung der modifizierten Regel

Aufstockungsfaktor fur Ansprtiche $B R_{t, t}<B R_{t}$ 
$\operatorname{co}(\omega)$

$C I$

$C V_{k, K}^{R}$

$d_{i}$

$d_{\Omega}(q s, z s)$

$\overline{d_{\Omega}^{\prime T}}(q s, z s)$

$\overline{d_{\Omega}^{I T}}(q s, z s)$

$\overline{d_{\Omega}^{I T_{1}, I T_{j}}}(q s, z s)$

$d_{p, q}$

$D M$

$D R_{k, K}$

EM, $E P_{i}^{(a)}$

$E P_{i}^{B I O}$

$E P_{i}^{G E S}$

$E P_{i}^{K I N D}$

$G E_{i}$

$i$

$i^{s}$

id
Kosten einer elementaren Operation $\varpi \in \Omega$

Eine Person

Barwert der Beitragszahlungen Person $k$ der Kohorte $K$ an ein Rentensystem $R$

Dummyvariable zur Kennzeichnung einer Eigenschaft der Person $i$

Kostenminimale Distanz zwischen den Sequenzen $(q s, z s)$

Durchschnittliche Distanz zwischen zwei beliebigen Sequenzen $q s, z s \in I T$

Durchschnittliche Distanz zwischen zwei beliebigen Sequenzen $q s, z s \in I T_{j}$

Durchschnittliche Distanz zwischen einer beliebigen Sequenz $q s \in I T_{i}$ und $z s \in I T_{j}: 1<j<k, i \neq j$

Kostenminimaler Distanzwert in der p-ten Zeile und der q-ten Spalte der Distanzmatrix $D M$

Eine Distanzmatrix

Zeitdauer über die eine typischen Person $k$ der Kohorte $K$ die Rentenlücke $P E_{k, K}^{G A P}$ schließen kann

Anzahl der Erwerbstätigen in einem Jahr $t$

Persönliche Entgeltpunkte einer Person $i$ im Alter von a Jahren

Gesamtanzahl an persönlichen Entgeltpunkten einer Person $i$ aus der Erwerbsbiographie

Gesamtanzahl an persönlichen Entgeltpunkten einer Person $i$

Persönliche Entgeltpunkte einer Person $i$ im Alter aufgrund der Kindererziehung

Gewicht einer Person $i$ in der Grundgesamtheit

Personenindex

Kapitalmarktzins in der Ansparphase

Kosten für Einfügen oder Löschen eines Elementes in der Quellsequenz 
$I M_{I T}$

IT

$I T_{j}$

$k$

$\mathrm{K}^{R}$

$\mathrm{K}_{t}^{R A E}$

$\mathrm{K}_{\text {, }}^{\mathrm{Re}}$

$\mathrm{K}_{\text {, }}^{\text {Vw }}$

$P_{i, t}(\lambda)$

$P^{B V}$

$P E_{k, K}^{G A P}$

$P E_{i, \ell}^{R}$

$P E_{k, K}^{R}$

qs

$P V_{k, K}^{R}$

$R$

$R F_{i}$

$R W_{1}$

$s$

$s b$

$S_{k, K}^{(a)}$

$t$

$t^{*}$

$T^{*}$
Menge der Individuen $i$ in der Gruppe $I T_{j} \subseteq I T$

Menge der individuellen Erwerbsbiographien

Teilmenge der individuellen Erwerbsbiographien

Typische Person

Ausgaben für Rentenzahlungen in einem Rentensystem $R$ im Jahr $t$

Aufwendungen für Rentenäquivalente im Jahr $t$

Aufwendungen für Rehabilitationsmaßnahmen im Jahr $t$

Verwaltungskosten im Jahr $t$

Wahrscheinlichkeit, dass ein beliebiges Individuum $i \mathrm{zu}$ einem Zeitpunkt $t$ eine Tätigkeit $\lambda$ ausübt

Ansprüche der Versorgungsempfänger

Rentenlücke einer typischen Person $k$ der Kohorte $K$

Monatsbetrag einer Rente an eine Person $i$ im Jahr $t$ in einem Rentensystem $R$

Monatsbetrag einer Rente aus privater Vorsorge einer

Person $k$ der Kohorte $K$

Eine Quellsequenz

Barwert der erwarteten Rentenzahlungen eines Rentensystems $R$ an eine Person $k$ der Kohorte $K$

Rentensystem $R \in\{B S, B R\}$

Rentenartfaktor einer Person $i$

Aktueller Rentenwert im Jahr $t$

Standardrentner

Kosten für Ersetzen eines Elementes in der Quellsequenz

Mögliche Sparleistung einer typischen Person $k$ der

Kohorte $K$

Jahresindex

Jahr der Systemumstellung

Das letzte Jahr der Systemumstellung 
Notwendige Gesamtkapital zur Deckung der Rentenlücke

$\mathrm{TK}^{R}$ Gesamtkosten eines Rentensystems $R$ im Jahr $t$

$T S_{k, K}^{\left(a^{\circ}\right)}$ Mögliche Sparkapital in der Erwerbsphase

$U F_{i, t}$ Überführungsbeträge einer Person $i$ im Jahr $t$

$V Z$ Maximale Versicherungszeit in den bestehenden Systemen

$V Z_{i}$ Versicherungszeit einer Person $i$

$w z_{i}$

$W Z$

Nachgewiesene Wartezeit einer Person $i$

$\mathrm{Y}$,

$Y_{k, K}^{(a)}$

Wartezeit ab der ein Anspruch auf Rentenleistung besteht

Durchschnittliches Einkommen aller sozialversicherungspflichtig Beschäftigten

Durchschnittliches Einkommen einer typischen Person $k$ der Geburtskohorte $K$ in einem Jahr $t=K+a$

$z s$

$\mathrm{Z}$,

$Z F_{i}$

Eine Zielsequenz

Zuschüsse des Bundes zur Finanzierung der Bürgerrente Zugangsfaktor einer Person $i$ 
7.5 Tabellen- und Abbildungsverzeichnis

\subsubsection{Tabellen}

Tabelle 1: $\quad$ Voraussetzungen zum Bezug Bürgerrente .............................21

Tabelle 2: Quantitative Analyse - Eine Übersicht .................................49

Tabelle 3: Kostensensitivität einer marginalen Veränderung der Regelaltersgrenze.

Tabelle 4: Kostensensitivität einer marginalen Veränderung des Startalters der Hinterbliebenensicherung .64

Tabelle 5: Kostensensitivität einer marginalen Veränderung der Wartezeitregelung .66

Tabelle 6: Kostensensitivität einer marginalen Veränderung der Wartezeitregelung bei einer Proratisierung der Rentenansprüche ....67

Tabelle 7: Kostensensitivität einer marginalen Veränderung des Leistungsniveaus

Tabelle 8: $\quad$ Kostenelastizitäten einzelner Determinanten ..........................69

Tabelle 9: $\quad$ Menge der Tätigkeiten........................................................72

Tabelle 10: Ermittlung der typischen Tätigkeiten am Beispiel des ersten Clusters in der Geburtskohorte 1974 ......................................83

Tabelle 11: Berechnungsgrundsätze der GRV-Renten.............................92

Tabelle 12: Berechnung der persönlichen Entgeltpunkte............................94

Tabelle 13: Beitragssätze in der Bürgerrente am Beispiel der Variante ALL/65/ALL18 ….................................................................96

Tabelle 14: Einkommen der Altersrentner (Cluster 1, Kohorte 1934).....112

Tabelle 15: $\quad$ Einkommen der Altersrentner (Cluster 3, Kohorte 1934).....114

Tabelle 16: Einkommen der Altersrentner, Finanztableau zum Zeitpunkt der Erhebung (Cluster 8, Kohorte 1944) ................................116

Tabelle 17: Einkommen der Altersrentner, Finanztableau zum Zeitpunkt des Eintritts in den Ruhestand (Cluster 8, Kohorte 1944).....117 
Tabelle 18: Einkommen der Altersrentner, Finanztableau zum Zeitpunkt der Erhebung (Cluster 9, Kohorte 1944) .............................. 118

Tabelle 19: $\quad$ Einkommen der Altersrentner, Finanztableau zum Zeitpunkt des Eintritts in den Ruhestand (Cluster 9, Kohorte 1944).... 120

Tabelle 20: Durchschnittlicher Pro-Kopf Saldo am Beispiel der Kohorte 1934

Tabelle 21: Entwicklung ausgewählter demographischer und ökonomischer Größen

Tabelle 22: Beitragssätze in der Bürgerrente am Beispiel der Variante SEL/65/SEL55

\subsubsection{Abbildungen}

Abbildung 1: Renditen eines Rentensystems im Vergleich - KDV versus GRV....

Abbildung 2: Möglichkeiten von systeminternen Leistungskumulationen in der GRV

Abbildung 3: Systemexterne Leistungskumulationen aufgrund eigener Ansprüche in den alten Bundesländern.

Abbildung 5: Kosten einer Bürgerrente im Vergleich zu den bestehenden

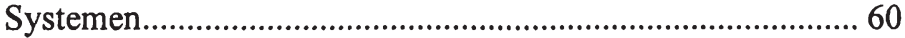

Abbildung 6: Tätigkeitsprofil einer Beispielperson..................................... 73

Abbildung 7: Distanzermittlung mit OMA................................................... 77

Abbildung 8: Clusteranzahl nach dem „Ellbow“-Kriterium am Beispiel der Geburtskohorte 1934 ...................................................... 79

Abbildung 9: Güte der Gruppierung am Beispiel der Kohorte 1934 ............. 80

Abbildung 10: Beispiel von fünf typischen Erwerbsverläufen in der Kohorte 1934 .....

Abbildung 11: Durchschnittlicher Einkommensverlauf eines typischen Individuums 90

Abbildung 12: Anpassung der Prognosewerte an die tatsächlichen Entgeltpunkte am Beispiel der Geburtskohorte 1934 104 
Abbildung 13: Preis eines temporären Abweichens von einer Normalerwerbsbiographie in der GRV...........................................108

Abbildung 14: Wirkungen eines temporären Abweichens von einer Normalerwerbsbiographie in der Bürgerrente.

Abbildung 15: Einkommen und Anteil der Gewinner in der Kohorte 1934.122

Abbildung 16: Einkommensveränderungen in der Kohorte 1934.

Abbildung 17: Wirkung der Erwerbseinkommen auf die Vorteilhaftigkeit einer Bürgerrente am Beispiel eines Standardrentners der Kohorte 1934.

Abbildung 18: Umverteilungen in den Rentensystemen...............................134

Abbildung 19: Die Entwicklung der Gesamtkosten eines Rentensystems.... 144

Abbildung 20: Wirkungen unterschiedlicher Zuwanderungssalden .............. 146

Abbildung 21: Struktur der Übergangsmodellierung für die GRV ...............152

Abbildung 22: Kostenpfade in der Übergangsphase ....................................157

Abbildung 23: Distanzermittlung mit der OMA nach Sankoff, Kruskal (1999) und Abbott, Hrycak (1990)..........................................169

Abbildung 24: Anteil der Gewinner in der Kohorte 1934 ..............................168

Abbildung 25: Einkommensveränderung in der Kohorte 1934.....................168

Abbildung 26: Rentenanwartschaften und Befragungspersonen....................169

Abbildung 27: Durchschnittlichen Rentenansprüche in der Übergangsphase

Abbildung 28: Entwicklung der durchschnittlichen Rentenansprüche der Altersrentner in einer Bürgerrente 


\subsection{Literaturverzeichnis}

AARON, HENRY J. (1966), The Social Insurance Paradox, in: Canadian Journal of Economics and Political Science, Vol. 32: 371-374.

ABBOT, ANDREW (1995), Sequence Analysis: New Methods for Old Ideas, in: Annual Review of Sociology, Vol. 21: 93-113.

AbBot, ANDrew, AlEXANDra HrycaK (1990), Measuring Resemblance in Sequence Data: An Optimal Matching Analysis of Musicians' Careers, in: American Journal of Sociology, Vol. 96, No. 1: 144-185.

Abbot, AndRew, Stanley DeViney (1992), The Welfare State as Transnational Event: Evidence from Sequences of Policy Adoption, in: Social Science History, Vol. 16, No. 2: 245-274.

ABBot, ANDREW, JOHN ForRest (1986), Optimal Matching Methods for Historical Sequences, in: Journal of Interdisciplinary History, Vol. 16, No. 3: 471-494.

ADAMSKI, KARSTEN J. (1999), Die Biedenkopfsche Konzeption der Bürgerrente, Diplomarbeit, Universität der Bundeswehr Hamburg, Hamburg.

AdAMSKI, Karsten J., GÖTZ Uebe (2001), Social Security, A micro data approach, Paper presented at the University of Lodz conference "Macromodels 2001", Krag, Poland.

AdAMSKI, Karsten J., Götz Uebe (2002C), Activity and Pension Profiles from the GSOEP, Paper presented at the University of Lodz conference "Macromodels 2002", Cedcyna, Polen, forthcoming in: Welfe, Wladyslaw (Ed.) (2003), Macromodels 2002, Modeling Economies in Transition, Lodz, Poland.

AdAMSKI, KARSTEN J. (2003A), Implications of a Transition to a Base Pension Scheme in Germany, Paper presented at the Hamburg Institute of International Economics, Hamburg, in: Discussion Papers in Statistics and quantitative Economics, Nr. 108, Universität der Bundeswehr Hamburg, Hamburg.

ADAMSKI, KARSTEN J. (2003B), Umstellung der Rentensysteme auf eine Grundrente, Eine quantitative Analyse eines Systemubergangs, in: Diskussionsbeiträge zur Statistik und quantitativen Ökonomik, Nr. 111, Universität der Bundeswehr Hamburg, Hamburg. 
AISENBREY, SilKe (2000), Optimal Matching Analyse, Anwendungen in den Sozialwissenschaften, in: Allmendinger, Jutta (Hrsg.), Studien zur Wissenschafts- und Organisationssoziologie, Bd. 2, Opladen: Leske und Budrich.

Bach, Stefan, Wolfgang Wiegard (2002), Public Economics, in: Zimmermann, Klaus F. (Ed.), Frontiers in Economics, Berlin: Springer, 37-94.

BÄCKER, GERHARD (2000), Bedürftigkeitsorientierte Grundsicherung im Alter. Element einer Rentenreform mit Zukunft? In: Soziale Sicherheit, Zeitschrift für Arbeit und Soziales, 49. Jg.: 42-47.

Bäcker, Gerhard, Reinhard Bispinck, Klaus HofemanN, Gerahrd NaEGELE (2000), Sozialpolitik und soziale Lage in Deutschland, Bd. 1+2, 3. Aufl., Wiesbaden: Westdeutscher Verlag.

Backhaus, Klaus, Bernd Erichson, Wulff Plinke, Rolf Weiber (2003), Multivariate Analysemethoden, Eine Anwendungsorientierte Einführung, 10. Aufl., Berlin: Springer.

Balasko, Yves, Karl Shell (1980), The Overlapping-generations Model, The Case of Pure Exchange without Money, in: Journal of Economic Theory, Vol. 23, No. 3: 281-306.

BARTH, HANS J. (1999), Langfristige Perspektiven der GRV vor dem Hintergrund politischer, wirtschaftlicher und demographischer Veränderungen, in: Allgemeines statistisches Archiv, Bd. 83: 45-54.

Besendorfer, Daniel, Cristoph BorgmanN, Bernd Raffelhüschen (1998), Ein Plädoyer für intergenerative Ausgewogenheit: Reformvorschläge auf dem Prüfstand, in: Diskussionsbeiträge, Institut für Finanzwissenschaft an der Universität Freiburg im Breisgau, Nr. 95.

Beveridge, William (1942), Social Insurance and Allied Services, Presented to Parliament by Command of His Majesty, His Majesty's Stationary Office, London. Dt. Fassung: Der Beveridgeplan, Sozialversicherung und verwandte Leistungen, Bericht von Sir William Beveridge, Dem Britischen Parlament überreicht im November 1992, Zürich: Europa Verlag, 1943.

BIEDENKOPF, KURT (1993), Eröffnungsrede der 25. Richterwoche des Bundessozialgerichtes am 18.10.1993 in Kassel. 
BiEdenKopf, Kurt, MeinhaRd Miegel (1997), Von der Arbeitnehmerrente zur Bürgerrente - Das Konzept der Grundsicherung im Alter für alle Bürgerinnen und Bürger, Manuskript, Dresden.

Boeri, Tito, AXel Börsch-Supan, Guido TABellini (2001), Would you like to shrink the welfare state? A Survey of European citizens, in: Economic Policy, No. 32: 9-44.

Bonin, Holger (2001), Generational Accounting, Theory and Application, Berlin: Springer.

BLÜM, NORBERT (1995), Langfristige Zukunftsperspektiven der Gesetzlichen Rentenversicherung, in: Konrad Adenauer Stiftung (Hrsg.), Langfristige Stabilisierung der gesetzlichen Rentenversicherung - Reformmodelle im Vergleich, Sankt-Augustin: 17-27.

BÖRSCH-SUPAN, AXEL (1998A), Übergangsmodelle vom Umlage- zum Kapitaldeckungsverfahren in der deutschen Rentenversicherung, in: Barbara Seel (Hrsg.), Sicherungssysteme in einer alternden Gesellschaft, Perspektiven sozialer Sicherung zwischen Selbstverantwortung und Solidarität, Frankfurt am Main: Campus,198-213.

BÖRSCH-SUPAN, AXEL (1998B), Germany: A Social Security System on the Verge of Collapse, in: Siebert, Horst (1998), (Ed.) Redesigning Social Security, Tübingen: Mohr Siebeck, 129-160.

BöRSCH-SUPAN, AXEL (1998C), Zur deutschen Diskussion eines Übergangs vom Umlage- zum Kapitaldeckungsverfahren in der gesetzlichen Rentenversicherung, in: Finanzarchiv, Bd. 55, Nr. 3: 399-428.

BÖRSCH-SUPAN, AXEL (2000A), Was lehrt uns die Empirie in Sachen Rentenreform, in: Perspektiven der Wirtschaftspolitik, Bd. 1, Nr. 4: 431-451.

BÖRSCH-SUPAN, AXEL (2000B), Soziale Sicherung: Herausforderungen an der Jahrhundertwende, in: Beiträge zur angewandten Wirtschaftsforschung, Nr. 590-00, Institut für Volkswirtschaftslehre und Statistik, Universität Mannheim.

BÖRSCH-SUPAN, AXEL (2001A), Blaupause für eine nachhaltige Rentenreform in Deutschland, in: Beiträge zur angewandten Wirtschaftsforschung, Nr. 599-00, Institut für Volkswirtschaftslehre und Statistik, Universität Mannheim.

BÖRSCH-SUPAN, AXEL (2001B), Was für die Kapitaldeckung und was für das Umlageverfahren spricht, in: Beiträge zur angewandten Wirtschafts- 
forschung, Nr. 587-00, Institut für Volkswirtschaftslehre und Statistik, Universität Mannheim.

BREYER, FrIEDRICH (1990), Ökonomische Theorie der Alterssicherung, in: Vahlens Handbücher der Wirtschafts- und Sozialwissenschaften, München: Vahlen.

BREYER, FRIEDRICH (2001), Why funding is not the Solution to the "Social Security Crisis", in: IZA Discussion Paper, No. 328, Bonn.

BREYER, FRIEDRICH (1994), Leitlinien für eine Systemkorrektur in der Rentenversicherung, in: Diskussionsbeiträge Serie I, Nr. 274, Fakultät für Wirtschaftswissenschaften und Statistik, Universität Konstanz.

BREYER, FRIEDRICH (1996), Zur Kombination von Kapitaldeckungs- und Umlageverfahren in der Deutschen Rentenversicherung, in: Gesellschaft für Versicherungswissenschaft und -gestaltung e.V. (Hrsg.), Die Alterssicherungssysteme vor der demographischen Herausforderung - Das Säulen-Modell der Weltbank als Lösungsansatz, Bonn, 115-138.

BREYER, FRIEDRICH (1998A), Individuelle und kollektive Sicherungsversprechen im demographischen Wandel, in: Barbara Seel (Hrsg.), Sicherungssysteme in einer alternden Gesellschaft, Perspektiven sozialer Sicherung zwischen Selbstverantwortung und Solidarität, Frankfurt am Main: Campus, 48-76.

Breyer, Friedrich (1998B), The Economics of Minimum Pensions, in: Siebert, Horst (1998), (Ed.) Redesigning Social Security, Tübingen: Mohr Siebeck, 273-294.

BREYER, FRIEDRICH (2000A), Kapitaldeckungs- versus Umlageverfahren, in: Perspektiven der Wirtschaftspolitik, Bd. 1, Nr 4: 383-405.

BREYER, FRIEDRICH (2000B), On the Intergenerational Pareto Efficiency of Pay-as-you-go Financed Pension Systems, in: Journal of Institutional and Theoretical Economics, Vol. 8, No. 145: 643-658.

BRUNNER, JOHANN K. (1994), Redistribution and the Efficiency of the Pay-asyou-go Pension System, in: Journal of Institutional and Theoretical Economies: 511-523.

BRUNNER, JOHANN K. (1998), Kapitaldeckungsverfahren versus Umlageverfahren: Grundsätzliches zur Systemdiskussion, in: Arbeitspapier, Nr. 9818, Sozial- und Wirtschaftswissenschaftliche Fakultät, Universität Linz-Auhof. 
BRUNNER, JOHANN K. (1999) Transfer zwischen den Generationen, in: Arbeitspapier, Nr. 9818, Sozial- und Wirtschaftswissenschaftliche Fakultät, Universität Linz-Auhof.

BUNDESMINISTERIUM DES INNERN, (2001A) (HRSG.), Entwicklung der Versorgungsausgaben seit 1970, URL: http://www.bmi.bund.de/dokumente/ artikel/ix51656.htm, 22.08.01.

BUNDESMINISTERIUM DES INNERN, (2001B) (HRSG.), Daten und Schlussfolgerungen zum Zweiten Versorgungsbericht der Bundesregierung, Das Wichtigste in Kürze, Berlin.

BUNDESMINISTERIUM FÜR ARBEIT UND SOZIALORDNUNG (1997), Vorschläge der Kommission „Fortentwicklung der Rentenversicherung“, Bonn.

BUNDESMINISTERIUM FÜR ARBEIT UND SOZIALORDNUNG (1998) (Hrsg.), Alterssicherungsbericht 1997, Bonn. Siehe auch: Deutscher Bundestag, Bundestags-Drucksache 13/9570, 30.12.1997.

BUNDESMINISTERIUM FÜR ARBEIT UND SOZIALORDNUNG (1999) (Hrsg.), Grundinformationen und Daten zur Sozialhilfe, Bonn.

BUNDESMINISTERIUM FÜR ARBEIT UND SOZIALORDNUNG (2001) (Hrsg.), Lebenslagen in Deutschland: Der erste Armuts- und Reichtumsbericht der Bundesregierung, Berlin.

BUNDESMINISTERIUM FÜR GESUNDHEIT UND SOZIALE SICHERUNG (2003) (Hrsg.), Sozial-Kompass Europa, Soziale Sicherheit in Europa im Vergleich, Bonn.

Burger, AleXANDer (1996), Deregulierungspotentiale in der Gesetzlichen Rentenversicherung, Reformnotwendigkeiten versus Reformmöglichkeiten, in: Hohenheimer Volkswirtschaftliche Schriften, Bd. 23, Frankfurt am Main: Peter Lang.

Burger, Alexander (1997), Zur Diskussion um eine Grundrente, In: Soziale Sicherheit, Zeitschrift für Arbeit und Soziales: 281-285.

Burgert, Derik, Hugo Dicke, Hans H. GlismanN, ERnst-Jürgen HoRn (1999), Ein quantitatives Szenario des Übergangs vom bestehenden System der Alterssicherung auf ein System der Grundsicherung für alle, in: Kieler Arbeitspapier Nr. 924, Institut für Weltwirtschaft, Kiel.

Buslei, HermanN, Florian Kraus (1996), Wohlfahrtseffekte eines graduellen Übergangs auf ein niedriges Rentenniveau, in :Steiner, Viktor, Klaus F. Zimmermann (Hrsg.), Soziale Sicherung und Arbeitsmarkt - 
Empirische Analyse und Reformansätze, ZEW-Wirtschaftsanalysen, Bd. 6, Baden-Baden: Nomos, 57-91.

BUTTLER, GÜNTER, NORBERT JÄGER, KARL RAPPL (1987), Was kostet die Einführung der Grundrente? in: Verband Deutscher Rentenversicherungsträger, Deutsche Rentenversicherung, Nr. 11-12: 748-762.

CHAN, TAK Wing (1995), Optimal Matching Analysis: A Methodological Note on Studying Career Mobility, in: Work and Occupations, Vol. 22, No. 4: $467-490$.

Clausing, Peter (1985), Auswirkungen des Grundrentenmodells von Miegel/ Wahl auf die Rentenversicherung, in: Die Angestelltenversicherung, 32. Jg.: 480-484.

DEUTSCHE BUNDESBANK (2002), Habenzinsen, Sparbriefe mit laufender Zinszahlung, vierjährige Laufzeit, Durchschnittssatz, Zeitreihe SU 0031.

DEUTSCHE BUNDESREGIERUNG (1996), Rentenversicherungsbericht 1996, in: Bundestagsdrucksache 13/5370, Bonn, 29.07.1996.

DEUTSCHE BUNDESREgIERUNG (1997A), Rentenversicherungsbericht 1997, in: Bundestagsdrucksache 13/8300, Bonn, 24.07.1997.

DEUTSCHE BUNDESREGIERUNG (1997), Alterssicherungsbericht 1997, in: Bundestagsdrucksache 13/9570, Bonn, 30.12.1997.

DEUTSCHE BUNDESREGIERUNG (1998A), Rentenversicherungsbericht 1998, in: Bundestagsdrucksache 13/11290, Bonn, 17.07.1998.

DEUTSCHE BUNDESREGIERUNG (1998B), (Hrsg.), Gesetz zu Korrekturen in der Sozialversicherung und zur Sicherung der Arbeitnehmerrechte, BGBL, Teil I 1998, Nr. 85, 28.12.1998, 3843-3852.

DEUTSCHE BUNDESREGIERUNG (1999A), Rentenversicherungsbericht 1999 und Gutachten des Sozialbeirates zum Rentenversicherungsbericht 1999, in: Bundestagsdrucksache 14/2116, Bonn, 02.12.1999.

DEUTSCHE BUNDESREGIERUNG (1999B), Lebenssituation von Seniorinnen und Senioren in der Bundesrepublik Deutschland, in: Bundestagsdrucksache 14/1717, Berlin, 06.10.1999.

DEUTSCHE BUNDESREGIERUNG (2001 A), Zweiter Versorgungsbericht der Bundesregierung, in: Bundestagsdrucksache 14/7220, Berlin, 19.10.2001. 
DEUTSCHE BUNDESREGIERUNG (2001B), Alterssicherungsbericht 2001, in: Bundestagsdrucksache 14/7640, Berlin, 23.11.2001.

DEuTSCHE BUNDESREGIERUNG (2001C) (Hrsg.), Gesetz zur Ergänzung des Gesetzes zur Reform der gesetzlichen Rentenversicherung und zur Förderung eines kapitalgedeckten Altersvorsorgevermögens (Altersvorsorgevermögensgesetz), in: BGBL, Teil I 2001, Nr. 13, 21.03.2001, 403-418.

DEUTSCHE BUNDESREGIERUNG (2002), Rentenversicherungsbericht 2002, in: Bundestagsdrucksache 15/110, Berlin, 21.11.2002.

DEUTSCHE BUNDESREGIERUNG (2003A), Nachhaltigkeitsfaktor soll der demographischen Entwicklung Rechnung tragen, Berlin, URL: http://www. Bundesregierung.de/Themen-A-Z/Rente-,474.490564/nachhaltigkeitsfak tor-soll-der.htm, 02.06.03.

DEUTSCHES INSTITUT FÜR AlteRSVORSORgE (1998A) (HRSG.), Reformvorschläge zur gesetzlichen Alterssicherung in Deutschland, Ein systematischer Überblick, Bonn.

DEUTSCHES INSTITUT FÜR ALTERSVORSORGE (1998B) (HRSG.), Effizienzvergleich, Renditen der gesetzlichen Rentenversicherung im Vergleich zu alternativen Anlageformen, Frankfurt am Main.

DICHMANN, WERNER (1994), Subsidiarität, Herkunft, sozialpolitische Implikationen und ordnungspolitische Konsequenzen eines Prinzips, in: Ordo, Jahrbuch für die Wirtschaft und Gesellschaft, Bd. 45: 195-249.

DIE GRÜNEN (1985), Grundrente statt Altersarmut, Antrag des Abgeordneten Bueb und der Fraktion Die Grünen, in: Bundestagsdrucksache 10/ 3496, Bonn, 18.06.1985.

Eckerle, Konrad, Michael Schlesinger, Gudrun Blaha (1995), PrognosGutachten 1995, Perspektiven der gesetzlichen Rentenversicherung für Gesamtdeutschland vor dem Hintergrund veränderter politischer und ökonomischer Rahmenbedingungen, in: VDR (Hrsg.) (1995), DRV-Schriften, Bd. 4, Frankfurt am Main. Zitiert als: Prognos (1995).

Eckerle, Konrad, Thomas OCZIPKa (1998), Prognos-Gutachten 1998, Auswirkungen veränderter ökonomischer und rechtlicher Rahmenbedingungen auf die gesetzliche Rentenversicherung in Deutschland, in: VDR (Hrsg.) (1998), DRV-Schriften, Bd. 9, Frankfurt am Main. Zitiert als: Prognos (1998). 
EITENMÜLLER, STEFAN (1996), Die Rentabilität der gesetzlichen Rentenversicherung - Kapitalmarktanaloge Renditeberechnungen für die nahe und ferne Zukunft, in: Deutsche Rentenversicherung, Nr. 12: 184-798.

EITENMÜLLER, STEFAN (2000), Reformoptionen für die gesetzliche Rentenversicherung: Auswirkungen der Rentenreform 2001 und die Verteilung der Umstiegskosten, Studie der PROGNOS AG, in: Edition HansBöckler-Stiftung Nr. 58, Düsseldorf. Zitiert als: Prognos (2000b).

EITENMÜLlER, STEFAN, KonRAD ECKERLE (2000), Umfinanzierung der Alterssicherung, Endbericht der PROGNOS AG im Auftrag der Hans-BöklerStiftung, in: Edition Hans-Böckler-Stiftung Nr. 33, Düsseldorf. Zitiert als: Prognos (2000a).

ERzBerger, Christian (2001), Sequenzmusteranalyse als fallorientierte Analysestrategie, in: Sackmann, Reinhold, Matthias Wingens (Hrsg.), Strukturen des Lebenslaufs, Übergang - Sequenz - Verlauf, in: Heinz, Walter R., Statuspassagen und Lebenslauf, Bd. 1, München, 135-162.

Feldstein, Martin, Elena Rangulova (2001), (Hrsg.), Individual risk in an investment-based social security system, in: Working Paper Series, No. 8074, Cambridge, Mass.

Feldstein, Martin, ANdrew Samwick (1998), The Transition Path in Privatizing Social Security, in: Feldstein, Martin (1998) (Eds.), Privatizing Social Security, Chicago, London, The University of Chicago Press, 215-265.

Fenge, Robert, (1995), Pareto-efficiency of the Pay-as-you-go Pension System with Intergenerational Fairness, in: Andel, Norbert et. al., Finanzarchiv, Bd. 52, Heft. 3: 357-363.

FENGE, ROBERT, (1997), Effizienz der Alterssicherung, in: Wissenschaftliche Beiträge, Nr. 149, Heidelberg: Physica.

FENGE, ROBERT, RoBert SCHWAGER (1995), Pareto-improving transition from a pay-as-you-go to a fully funded pension system in a model with differing earning abilities, in: Zeitschrift für Wirtschafts- und Sozialwissenschaften, Nr. 115: 367-376.

FENGE, RoBert, JACOB VON WEIZSÄCKER (1999), To what extent are public pensions pareto-improving? On the interaction of means tested basic 
income and public pensions, in: CESifo Working Paper Series, No. 197: 2-23.

Fleck, Claudia (1996), Konzeption eines Mikrosimulationsmodells für die Bereiche 'Demographie' und 'Erwerbsbeteiligung' auf der Grundlage des Sozioökonomischen Panels, Dissertation, Darmstadt.

GlismanN, Hans H. (1997), Aufwand und Ertrag in der deutschen gesetzlichen Alterssicherung (GRV), in: Kieler Arbeitspapier Nr. 840, Institut für Weltwirtschaft, Kiel.

HÄRTEL, HANS-HAGEN, (2001), Schutz der Ehe noch zeitgemäß? In: Wirtschaftsdienst, Nr. 8: 424

HAISKEN-DeNew, John P., JoAChIM R. Frick (HRSG.) (2000), DTC, Desktop Companion to the German Socio-Economic Panel Study (GSOEP), Version 4.0, Berlin.

HalPin, Brendan, TaK Wing Chan (1998), Class Careers as Sequences: An Optimal Matching Analysis of Work-Life Histories, in: European Sociological Rewiew, Vol. 14, No. 2: 111-130.

HAUENSCHILD, NiLS (2000), Pareto-improving Transition from Pay-as-you-go to Fully Funded Social Security under Uncertain Incomes, in: Finanzarchiv, Bd. 57, Nr. 1: 39-62.

HAUSER, RICHARD (1993), Mindestsicherung im Alter, Ausgewählte ökonomische Aspekte unter Berücksichtigung von Wanderungen, in: Schmähl, Wilfried (1993) (Hrsg.), Mindestsicherung im Alter: Erfahrungen, Herausforderungen, Strategien, Schriften des Zentrums für Sozialpolitik, Bd. 2, Frankfurt am Main: Campus, 75-99.

HAUSER, RiCHARD (1996), Ziele und Möglichkeiten einer Sozialen Grundsicherung, in: Schriftenreihe „Dialog Sozial“, Bd. 1, Baden-Baden: Nomos.

HAUSER, RICHARD (2000), Bedarfsorientierte Mindestsicherung: Einbau in die Rente?, in: Soziale Sicherheit, Zeitschrift für Arbeit und Soziales, 49. Jg.: 187-192.

HEUbECK, KLAUS, BERT RÜRUP (2000), Finanzierung der Altersversorgung des öffentlichen Dienstes, Probleme und Optionen, Gutachten im Auftrag der Arbeitsgemeinschaft kommunale und kirchliche Altersversorgung (AKA) e.V., in: Rürup, Bert (Hrsg.) Sozialökonomische Schriften, Bd. 20, Frankfurt am Main: Peter Lang. 
Hirte, Georg, Reinhard Weber (1997), Pareto improving Transition from a Pay-as-you-go to a Fully Funded System - is it Politically Feasible?, in: Finanzarchiv, Bd. 54, Heft. 3: 303-330.

Homburg, Stefan (1987), Theorie der Alterssicherung, in: Studies in Contemporary Economics, Berlin: Springer.

Homburg, STEFan (1990), The Efficiency of Unfunded Pension Schemes, in: Journal of Institutional and Theoretical Economics, No 146: 640-647.

Homburg, Stefan (2000), Ein Schnellkurs in Sachen Rentenreform, in: Perspektiven der Wirtschaftspolitik, Nr. 4: 379-381.

HÜTHER, MiCHAEL (1998), Rezension zu Hauser, Richard (1996), Ziele und Möglichkeiten einer Sozialen Grundsicherung, in: Finanzarchiv, Bd. 55, Nr. 2: 270-274.

INFRATEST BURKE SOZIALFORSCHUNG (2000), SOEP'99, Methodenbericht zum Befragungsjahr 1999 (Welle 16) des sozio-oekonomischen Panels.

JÄHRLING-RAHNEFELD, BRIGITTE (2002), Verfassungsmäßigkeit der Grundrente, Verfassungsprobleme eines Systemwechsels in der gesetzlichen Rentenversicherung in: Europäische Hochschulschriften, Reihe II Rechtswissenschaft, Bd. 3434, Frankfurt am Main: Peter Lang.

JAMES, ESTELLE (1996), The world bank's three-pillar system: Will it provide income security to the world's aging population? In: Gesellschaft fur Versicherungswissenschaft und -gestaltung e.V. (Hrsg.), Die Alterssicherungssysteme vor der demographischen Herausforderung - Das Säulen-Modell der Weltbank als Lösungsansatz, Bonn, 43-62a.

JeSS, HeINRICH (1998), Das Prognos-Gutachten 1998, Ausgewählte Ergebnisse und Erläuterungen, in: Die Angestellten Versicherung, Nr. 9: 353-368.

Koch, Achim, Martina Wasmer, Janet Harkness, Evi Scholz (2001), Konzeption und Durchführung der "Allgemeinen Bevölkerungsumfrage der Sozialwissenschaften" (ALLBUS) 2000, in: ZUMA-Methodenbericht Nr. 5, Mannheim

KOMMISSION FÜR DIE NACHHALTIGKEIT IN DER FINANZIERUNG DER SOZIALEN SICHERUNGSYSTEME (2003A) (Hrsg.), Rürup-Kommission legt Vorschläge zum Rentensystem vor, Berlin. 
KOMMISSION FÜR DIE NACHHALTIGKEIT IN DER FINANZIERUNG DER SOZIALEN SiCHERUNGSYSTEME (2003B) (Hrsg.), Bericht der Kommission, Berlin.

KOMMISSION „SOZIALE SICHERHEIT“ (2003) (Hrsg.), Bericht der Kommission „Soziale Sicherheit" zur Reform der sozialen Sicherungssysteme, Berlin.

KotLIKOFF, LAURENCE J. (1998), Simulating the Privatization of Social Security in General Equilibrium, in: Privatizing Social Security, Chicago: The University of Chicago Press, 265-306.

KÖPP, GÜNTHER (1995), Probleme der Rentenfinanzierung, Bern: Haupt.

KÖPP, GÜNTHER, GÖTZ UEBE (1994), Long run simulation of German social security system, in: Bachem Achim, Ulrich Derigs, Michael Jünger, Rainer Schrader, (eds.), Operations Research '93, Heidelberg: Physica, 300-303.

KiffManN, Mathias (2001), Langfristige Folgen einer Einbeziehung der Selbständigen in die gesetzliche Rentenversicherung, in: DIW Diskussionspapier Nr. 251, Berlin.

KIRNER, ELLEN (1987), Keine Mindestrente für die Armen, weil Sie nichts geleistet haben, in: Deutsches Zentrum für Altersfragen (Hrsg.), Die ergraute Gesellschaft, Berlin, 243-262.

Klanberg, Frank, Alois Prinz (1988), Perspektiven der Mindestsicherung, in: Sozialpolitische Schriften, Nr. 58, Berlin.

KRUPP, HANS-JÜRgen, JOACHIM WeEBer (1997), Pro und Contra - Eine Analyse aus volkswirtschaftlicher Sicht, in: Verband Deutscher Rentenversicherungsträger, Nr. 3-4, 205-219.

LANGEN, HANS-GEORG (2001), Die Renteninformation: Ein neues Medium für die Versicherten der gesetzlichen Rentenversicherung, in: Die Angestelltenversicherung, Jg. 48, Nr. 10: 1-8.

Levensthein, VladimiR I. (1966), Binary Codes Capable of Correcting Deletions, Insertions and Reversals, in: Cybernetics and Control Theory, No. 10: 707-710.

LEYENDECKER, WOLFGANG (1986), Rentenmodell für die Zukunft? In: Verband Deutscher Rentenversicherungsträger (Hrsg.), Deutsche Rentenversicherung, Nr. 3-4, 143-160.

Ludwig, AleXANDER (2002), Rentenreform im globalen Kontext, Ein Multi-Regionen-Simulationsmodell, in: Europäische Hochschulschriften, Reihe 
V, Volks- und Betriebswirtschaft, Bd. 2819, Frankfurt am Main: Peter Lang.

LÜTH, ERIK (2000), Private Intergenerational Transfers and Population Aging: The German Case, Dissertation, Universität Freiburg (Breisgau), Heidelberg: Physica.

MEZGER, ERIKA, (Hrsg.) (2000), Zukunft der Alterssicherung, Tagung am 23./ 24. September 1999 in Berlin, in: Edition Hans-Böckler-Stiftung Nr. 28, Düsseldorf.

Miegel, Meinhard (1981), Sicherheit im Alter, Plädoyer für die Weiterentwicklung des Rentensystems, in: Schriften des Instituts für Wirtschafts- und Gesellschaftspolitik, Stuttgart.

Miegel, Meinhard, Stefanie Wahl (1985), Gesetzliche Grundsicherung, Private Vorsorge, Der Weg aus der Rentenkrise, Stuttgart: Bonn Aktuell.

Miegel, Meinhard, Stefanie Wahl (1999), Solidarische Grundsicherung, Private Vorsorge, Der Weg aus der Rentenkrise, München: Aktuell im Olzog Verlag.

MitsChKE, JoACHIM (2000), Grundsicherungsmodelle - Ziele, Gestaltung, Wirkungen und Finanzbedarf, Eine Fundamentalanalyse mit besonderem Bezug auf die Steuer- und Sozialordnung sowie den Arbeitsmarkt der Republik Österreich, Baden-Baden: Nomos.

MÜLlER, UlRICH (2000), Maßzahlen der Bevölkerungsstatistik, in: Müller, Ulrich, Berhard Nauck, Andreas Diekmann (Hrsg.), Handbuch der Demographie 1, Modelle und Methoden, Berlin: Springer, 1-91.

Müller, Hans-Wolf, Roland Tautz (1996), Ein Grundrentensystem ist teuer, in: Verband Deutscher Rentenversicherungsträger, Deutsche Rentenversicherung, Nr. 12, 770-783.

NEUMANN, MANFRED (1997), Vom Umlageverfahren zum Kapitaldeckungsverfahren: Optionen zur Reform der Alterssicherung, in: Rentenkrise. Und wie wir sie meistern können. Frankfurter Institut, Kleine Standbibliothek Nr. 21, Frankfurt am Main, 87-128.

Neumann, Manfred (1998), Replacing Germany's Public Old Age Pension System with a Fully Funded System, in: Morath, Konrad (Hrsg.) (1998), Verläßliche soziale Sicherung, Frankfurter Institut, Stiftung Marktwirtschaft und Politik, Frankfurt am Main, 53-63. 
OhSmanN, SaBINe, UlRich Stolz (1997), Beitragszahlungen haben sich gelohnt. Betrachtungen zur Rendite der Altersrenten in der gesetzlichen Rentenversicherung, in: Deutsche Angestelltenversicherung, 119-124.

Pestiau, Pierre (2000), Investing Social Security in the Equity Market. Does it Make a Difference, In: National Tax Journal, Vol. LIII, No. 1: 4157.

PfaFf, ANITA B. (1999), Veränderte Erwerbsbiographien und ihre Auswirkungen auf die Sozialpolitik, in: Wandel der Arbeitswelt - Folgerungen für die Sozialpolitik, Baden-Baden: Nomos, 31-60.

PISCHNER, RAINER (2000), Überarbeitete Querschnittshochrechnung der Wellen G-N (1990 bis 1997) des Sozioökonomischen Panels (SOEP) unter Einbeziehung der Ergänzungsstichprobe E (Welle O), Berlin.

Prognos (1998), siehe Eckerle (1998).

RAFFELHÜSCHEN, BERND (1993), Funding Social Security Through Paretooptimal Conversion Policies, in: Felderer, Bernhard (Ed.), Journal of Economics, S. 7, Wien: Springer, 105-131.

RAFFELHÜSCHEN, BERND, ALF E. RISA (1995), Reforming social security in a small open economy, in: European Journal of Political Economy, Vol. 11: 469-485.

RAHN, MONIKA (1999), Reform der gesetzlichen Rentenversicherung in Deutschland aus rechtsvergleichender Sicht, in: Verband Deutscher Rentenversicherungsträger, Deutsche Rentenversicherung, Nr. 12, 377-409.

RAwLS, JoHN (1971), A Theory of Justice, Cambridge, Mass.: Havard University Press. Dt. Fassung: Rawls John (1975), Eine Theorie der Gerechtigkeit, Frankfurt am Main: Suhrkamp.

ROMER, PAUL M. (1986), Increasing Returns and Long-Run Growth, in: Journal of Political Economy, Vol. 94, No. 5: 1002-1037.

ROHWER, GÖTZ, UlRICH PÖTTER (1999), TDA Users Manual, Bochum.

REINHARD, HANS-JOACHIM (2000), Umverteilung und Grundrente - Frauen und Alterssicherung in den USA und Kanada, in: Zeitschrift für Sozialreform: 762-778.

RULAND, FranZ (1998), Die Zukunft der Rentenversicherung, in: Jörg-E. Cramer et. al. (Hrsg.), Handbuch zur Altersvorsorge: gesetzliche, betriebliche und private Vorsorge in Deutschland, Frankfurt a.M.: Knapp, 935-972. 
RULAND, FRANZ (1999), Contra: Bedürftigkeitsorientierte Mindestsicherung, in: Deutsche Rentenversicherung, No. 8-9, 480-493.

RULAND, Franz (2000), Einstieg in den Systemwechsel, in: Soziale Sicherheit, Jg. 49, Nr. 2: 38-41.

RÜRUP, BerT (2002), The German Pension system: Status quo and Reform Options, in: Feldstein, Martin, Horst Siebert (Hrsg.) (2002), Social Security Pension Reform in Europe, Chicago: University of Chicago Press, 137-163.

SACHVERSTÄNDIGENRAT ZUR BEGUTACHTUNG DER GESAMTWIRTSCHAFTLICHEN ENTWICKLUNG (1998), Vor weitreichenden Entscheidungen, Jahresgutachten 1998/99, Stuttgart: Metzler-Poeschel.

SACHVERSTÄNDIGENRAT ZUR BEGUTACHTUNG DER GESAMTWIRTSCHAFTLICHEN ENTWICKLUNG (2001), Für Stetigkeit - Gegen Aktionismus, Jahresgutachten 2001/02, Stuttgart: Metzler-Poeschel.

SAMUELSON, PAUL A. (1958), An exact consumption-loan model of interest with or without the social contrivance of money, in: The Journal of Political Economy, Vol. 66, No. 6: 467-482.

SANKhoff, David, Joseph Kruskal (1999), Time Wraps, String Edits, and Macromolecules, The Theory and Practice of Sequence Comparison, reissue ed., 1. print (1983), Stanford, Calif.: Addison-Wesley.

SCHACHT, UlRICH (2001), Finanztheoretische Analyse der Alterssicherung, in: Europäische Hochschulschriften, Reihe V, Volks- und Betriebswirtschaft, Bd. 2793, Frankfurt am Main: Peter Lang.

SCHAEPER, HILDEGARD (1999), Erwerbsverläufe von Absolventinnen und Absolventen: Eine Anwendung der Optimal Matching Technik, in: Arbeitspapier, Nr. 57, Sonderforschungsbereich 186 der Universität Bremen: Statuspassagen und Risikolagen im Lebensverlauf.

SCHMÄHL, WINFRIED (1974), Systemänderung in der Altersvorsorge, Von der einkommensabhängigen Altersrente zur Staatsbürger-Grundrente. Eine theoretische und empirische Untersuchung ökonomischer Probleme im Übergangszeitraum, Opladen: Westdeutscher Verlag.

SCHMÄHL, WINFRIED (1986), Ökonomische Probleme des Übergangs zu einem Grundrentenmodell, in: Die Angestelltenversicherung, Nr. 4: 122-127. 
SCHMÄHL, WINFRIED (1988), Übergang zu Staatsbürger-Grundrenten, Ein Beitrag zur Deregulierung in der Alterssicherung, in: Thiemeyer, Theo (Hrsg.), Regulierung und Deregulierung im Bereich der Sozialpolitik, Schriften des Vereins für Sozialpolitik, N.F., Bd. 177, Berlin: Duncker \& Humblot, 83-138. Zitiert nach: Schmähl, Winfried (1993) (Hrsg.), Mindestsicherung im Alter: Erfahrungen, Herausforderungen, Strategien, Schriften des Zentrums für Sozialpolitik, Bd. 2, Frankfurt am Main: Campus, 263-333.

SCHMÄHL, WINFRIED (1993), Alternative Strategien für die Mindestsicherung im Alter in Deutschland, Arbeitspapier, Nr. 92, Zentrum für Sozialpolitik, Bremen.

SCHMÄHL, WINFRIED (1996), Die Alterssicherung aus gesamtwirtschaftlicher und ordnungspolitischer Sicht, In: Gesellschaft für Versicherungswissenschaft und -gestaltung e.V. (Hrsg.), Die Alterssicherungssysteme vor der demographischen Herausforderung - Das Säulen-Modell der Weltbank als Lösungsansatz, Bonn, 19-41.

SCHMÄHL, WiNfRIED (1998), Perspektiven der Alterssicherung - Anmerkungen zu Konzeptionen und Instrumenten, in: Barbara Seel (Hrsg.), Sicherungssysteme in einer alternden Gesellschaft, Perspektiven sozialer Sicherung zwischen Selbstverantwortung und Solidarität, Frankfurt am Main: Campus, 154-181.

SCHMÄHL, WINFRIED (1999), Steigende Lebenserwartung und soziale Sicherung - Tendenzen, Auswirkungen und Reaktionen, in: Arbeitspapier, Nr. 4, Zentrum für Sozialpolitik, Bremen.

SCHMÄHL, WINFRIED (2000A), Perspektiven der Alterssicherungspolitik in Deutschland - Über Konzeptionen, Vorschläge und einen angestrebten Paradigmenwechsel, in: Perspektiven der Wirtschaftspolitik, Bd. 1, Nr. 4: 407-430.

SCHMÄHL, WINFRIED (2000B), Alterssicherung von Frauen im Prozess ökonomischen, gesellschaftlichen und politischen Wandels - zugleich zur Einführung in das Thema des Kolloquiums, in: Schmähl, Winfried, Klaus Michaelis (Hrsg.), Alterssicherung von Frauen, Leitbilder, gesellschaftlicher Wandel und Reformen, Wiesbaden: Westdeutscher Verlag, 9-35. 
SCHMÄHL, WINFRIED (2001) (HRSG.), Wechselwirkungen zwischen Arbeitsmarkt und sozialer Sicherung, in: Schriften des Vereins für Sozialpolitik, Bd. 285, Berlin: Duncker \& Humblot.

SCHMÄHL, WINFRIED (2003) (HRSG.), Soziale Sicherung und Arbeitsmarkt, in: Schriften des Vereins für Sozialpolitik, Bd. 294, Berlin: Duncker \& Humblot.

SCHUlte, BERND (1993), Praktizierte Formen der Mindestsicherung im Alter ein rechtsvergleichender Überblick, in: Schmähl, Winfried (1993) (Hrsg.), Mindestsicherung im Alter: Erfahrungen, Herausforderungen, Strategien, Schriften des Zentrums für Sozialpolitik, Bd. 2, Frankfurt am Main: Campus, 27-74.

Siebert, Horst (1998), Pay-as-You-Go versus Capital-Funded Pension System: The Issues, in Siebert, Horst (1998) (Ed.), Redesigning Social Security, Tübingen: Mohr Siebeck, 3-34.

SinN, HANS-Werner, MARCEl Thum (1999), Gesetzliche Rentenversicherung: Prognosen im Vergleich, in: Ifo Diskussionsbeiträge, Nr. 62, Ifo-Institut für Wirtschaftsforschung, München.

Simon, Sivlia (2001), Umverteilung in der Sozialversicherung, Begründung, Analyse und Bewertung in gegenwarts- und zukunftsorientierter Perspektive, in: Europäische Hochschulschriften, Reihe V, Volks- und Betriebswirtschaft, Bd. 2714, Frankfurt am Main: Peter Lang.

SoEP Group (2000), (HrSG.), Benutzerhandbuch, Fragebögen, Welle A bis Z, Berlin.

Soep Group (2001 A), The German Socio-Economic Panel (GSOEP) after more than 15 years - Overview, in: Holst, Elke, Dean R. Lillard, Thomas A. DiPrete (Hrsg.), Proceedings of the 2000 Fourth International Conference of German Socio-Economic Panel Study Users (GSOEP 2000), Vierteljahrshefte zur Wirtschaftsforschung, Vol. 70, No. 1: 7-14.

SOEP GROUP (2001 B), Leben in Deutschland, Berlin.

SozialbeiRat (1996), Gutachten des Sozialbeirates zum Rentenversicherungsbericht 1996, in: Bundestagsdrucksache 13/5370, 29.07.96.

Sozialbeirat (1997), Gutachten des Sozialbeirates zu den mittel- und langfristigen Vorausberechnungen des Rentenversicherungsberichtes 1997, in: Bundestagsdrucksache 13/8300, 24.07.97, 217-228. 
SOZIALBEIRAT (1998), Gutachten des Sozialbeirates zum Rentenversicherungsbericht 1998 und Stellungsnahme zu einigen weiteren Berichten zur Alterssicherung, in: Bundestagsdrucksache 13/11290, 17.07.98, 239252.

SOZIALBEIRAT (1999), Gutachten des Sozialbeirates zum Rentenversicherungsbericht 1999, in: Bundestagsdrucksache 14/2116, 02.12.99, 131-148.

SOZIALBEIRAT (2001), Sondergutachten des Sozialbeirates zur Rentenreform, in: Bundestagsdrucksache 14/5394, 13.02.01.

SOZIALBEIRAT (2002), Gutachten des Sozialbeirates zum Rentenversicherungsbericht, in: Bundestagsdrucksache 15/110, 27.11.02, 121-140.

SPIESS, MARTIN (2000), Derivation of design weights: The case of the German Socio-Economic Panel (GSOEP), Berlin.

SPREMANN, Klaus (1984), Intergenerational Contracts and their Decomposition, in: Journal of Economics, Vol. 44: 237-253.

Statistisches Bundesamt (LFD. JAHRE A), (Hrsg.) Statistisches Jahrbuch, Wiesbaden.

STATISTISCHES BUNDESAMT (LFD. JAHRE B), Bevölkerung und Erwerbstätigkeit, Fachserie 1, Reihe 3, Wiesbaden.

STATISTISCHES BundesAMT (LFD. JAHRE C), Finanzen und Steuern, Fachserie 14, Reihe 6.1, Versorgungsempfänger des öffentlichen Dienste, Wiesbaden.

STATistisches Bundesamt (LFD. JAhre D), Sozialleistungen, Fachserie 13, Reihe 2, Sozialhilfe, Wiesbaden.

STATISTISCHES BUNDESAMT (LFD. JAHRE E), Bevölkerung und Erwerbstätigkeit, Fachserie 1, Reihe 4.2.2, Wiesbaden.

STATISTISCHES BUNDESAMT (2000A), Pflegestatistik über stationäre Einrichtungen, Pflegebedürftige nach Art und Träger der Einrichtung Fachserie 4, Reihe 1, Wiesbaden.

STATISTISCHES BUNDESAMT (2000B), Weitere Zunahme der Lebenserwartung, URL: http://www.destatis.de/presse/deutsch/pm/p0aa7022htm.htm, 23.07.02.

STATISTISCHES BUNDESAMT (2000C), Bevölkerungsentwicklung Deutschlands bis 2050, Ergebnisse der 9. koordinierten Bevölkerungsvorausberechnung, CD-Rom, Wiesbaden. 
STATISTISChEs BundeSAMT (2003), Bevölkerung Deutschlands bis 2050, 10. koordinierte Bevölkerungsvorausberechnung, CD-ROM, Wiesbaden.

STEINIGER, Christian (1996), Grundsicherung als sozialpolitische Alternative?, in: Institut für Gesellschaftswissenschaften (Hrsg.), Die Neue Ordnung, Jg. 50, Nr. 3: 202-210.

StRAUBhaAR, THOMAS (2000), Wieso nicht Rente mit 67? In: Wirtschaftsdienst, Jg. 2000, Nr. 9: 518-519.

StraubhaAR, Thomas (2001), East-West Migration: Will it be a Problem? In: Intereconomics, Vol. 36, No. 4: 167-170.

SÜDDEUTSCHE ZEITUNG (2000) Grünen-Sozialexpertin für radikale Rentenreform, 27.03.2000.

SÜDDEUTSCHE ZEITUNG (2003A), Rürup-Plan: Rente erst ab 67, 24.04.03

SÜDDEUTSCHE ZEITUNG (2003B), Erst mit 70 Jahren in die Rente, 03.07.03.

SÜdDEUTSCHE ZEITUNG (2003C), Euro um Euro, Zahn um Zahn, 21.07.03.

VERBAND DEUTSCHER RENTENVERSICHERUNGSTRÄGER (LFD. JAHRE), (Hrsg.), VDR-Statistik, Rentenbestand am 31. Dezember [..], (Lfd. Bände), Frankfurt am Main.

VERBAND DEUTSCHER RENTENVERSICHERUNGSTRÄGER (1997), Verband Deutscher Rentenversicherungsträger lehnt Biedenkopfs „Bürgerrente“ ab, URL: http://www.vdr.de, 16.07.2002.

VERBAND DEutScher RENTENVERSICHERUNGSTRÄGER (2000), (Hrsg.), Rentenversicherung in Zeitreihen, DRV-Schriften Bd. 22, Frankfurt am Main.

VERBAND DEUTSCHER RENTENVERSICHERUNGSTRÄGER (2002), VDR-Statistik, Rentenzugang, Bd. 145, Frankfurt am Main.

Verband Deutscher RentenVersicherungsträger (2003), Ausgaben RV, URL: http://www.vdr.de, 24.07.03.

WAHL, STEFANIE (1995), Übergang zu einer steuerfinanzierten StaatsbürgerGrundsicherung, in: Konrad Adenauer Stiftung (Hrsg.), Langfristige Stabilisierung der gesetzlichen Rentenversicherung - Reformmodelle im Vergleich, Sankt-Augustin, 72-79. 
WAHL, StEFANIE (1999), Wege aus der Rentenkrise, in: Ludwig Erhard Stiftung (Hrsg.), Orientierungen zur Wirtschafts- und Gesellschaftspolitik 80 , Nr. 2, Bonn.

WeizsäCKer, CARL Christian (1979), Das eherne Zinsgesetz, Kyklos, 32, 270-288.

WESTDEUTSCHE LANDESBANK (2002), Zur aktuellen Zinsentwicklung, Nr. 32.

WigGer, BERTHOLD U. (2001), Pareto-improving intergenerational transfers, in: Oxford Economic Papers, Vol. 53, No. 2: 260-280.

WiNDIZIO, MichaEL (2001), Übergänge und Sequenzen, Der Einfluss von Arbeitslosigkeit auf den weiteren Erwerbsverlauf, in: Sackmann, Reinhold, Wingens, Matthias (Hrsg.), Strukturen des Lebenslaufs, Übergang - Sequenz - Verlauf, Weinheim: Juventa, 163-198.

WiRTSCHAFTSJUNIOREN (2002), Ergebnisse Bevölkerungsbefragung 2002, Manuskript.

WISSENSCHAFTLICHER BEIRAT BEIM BUNDESMINISTERIUM FÜR WIRTSCHAFT (1998), Grundlegende Reform der gesetzlichen Rentenversicherung, Gutachten vom 20./21. Februar 1998, BMWi-Studienreihe 99.

WORLD BANK (1994), Averting the old Age Crisis: Policies to protect the old and promote growth, Oxford: Oxford University Press. 


\section{SCHRIFTEN ZUR WIRTSCHAFTSTHEORIE UND WIRTSCHAFTSPOLITIK}

Herausgegeben von Rolf Hasse, Jörn Kruse, Wolf Schăfer, Thomas Straubhaar, Klaus W. Zimmermann

Band 1 Lars Bünning: Die Konvergenzkriterien des Maastricht-Vertrages unter besonderer Berũcksichtigung ihrer Konsistenz. 1997.

Band 2 Andreas Henning: Beveridge-Kurve, Lohnsetzung und Langzeitarbeitslosigkeit. Eine theoretische Untersuchung unter Berücksichtigung des Insider-Outsider-Ansatzes und der Entwertung des Humankapitals. 1997.

Band 3 Iris Henning: Die Reputation einer Zentralbank. Eine theoretische Untersuchung unter besonderer Berücksichtigung der Europäischen Zentralbank. 1997.

Band 4 Rüdiger Hermann: Ein gemeinsamer Markt für Elektrizităt in Europa. Optionen einer Wettbewerbsordnung zwischen Anspruch und Wirklichkeit. 1997.

Band 5 Alexander Tiedtke: Japan und der Vorwurf des Trittbrettfahrerverhaltens in der US-amerikanisch-japanischen Allianz. 1997.

Band 6 Wolfgang Grimme: Ordnungspolitisches Konzept der Regionalpolitik. Darstellung der Defizite und des Reformbedarts der Regionalpolitik am Beispiel Mecklenburg-Vorpommems. 1997.

Band 7 Christian Ricken: Determinanten der Effektivităt der Umweltpolitik. Der nationale Politikstil im Spannungsfeld von Ökonomie, Politik und Kultur. 1997.

Band 8 Christian Schmidt: Real Convergence in the European Union. An Empirical Analysis. 1997.

Band 9 Silvia Marengo: Exchange Rate Policy for MERCOSUR: Lessons from the European Union. 1998.

Band 10 Jens Kleinemeyer. Standardisienung zwischen Kooperation und Wettbewerb. Eine spieltheoretische Betrachtung. 1998.

Band 11 Stefan M. Golder. Migration und Arbeitsmarkt. Eine empirische Analyse der Performance von Auslăndem in der Schweiz. 1999.

Band 12 Stefan Kramer. Die Wirkung einer Internationalisierung des Yen auf die japanischen Finanzmărkte, die japanische Geldpolitik und die Usancen der Fakturienung. 1999.

Band 13 Antje Marielle Gerhold: Wirtschaftliche Integration und Kooperation im asiatisch-pazifischen Raum. Die APEC. 1999.

Band 14 Tamim Achim Dawar: Deutsche Direktinvestitionen in Australien. Eine Evaluation der theoretischen Erklărungsansătze und der Standortattraktivităt des Produktions- und Investitionsstandortes Australien. 1999.

Band 15 Hans-Markus Johannsen: Die ordnungspolitische Haltung Frankreichs im Prozeß der europăischen Einigung. 1999.

Band 16 Annette Schönherr: Vereinigungsbedingte Dimensionen regionaler Abeitsmobilităt. Wirkungen unter analytisch einfachen Bedingungen und potentielle individuelle Migrationsgewinne in Deutschland nach der Vereinigung. 1999.

Band 17 Henrik Müller. Wechselkurspolitik des Eurolandes. Konfliktstoff für die neue wăhrungspolitische Ära. 1999.

Band 18 Lars H. Wengorz: Die Bedeutung von Unternehmertum und Eigentum für die Existenz von Unternehmen. Eine methodenkritische Analyse der Transformation des Wirtschaftssystems in Russland. 2000.

Band 19 Eckart Bauer: Konzeptionelle Grundfragen eines Kinderleistungsausgleichs im Rahmen einer umlagefinanzierten zwangsweisen Rentenversicherung. 2000. 
Band 20 Hubertus Hille: Enlarging the European Union. A Computable General Equilibrium Assessment of Different Integration Scenarios of Central and Eastern Europe. 2001.

Band 21 Tobias Just: Globalisierung und Ideologie. Eine Analyse der Existenz und Persistenz von Partisaneffekten bei zunehmender Intemationalisienung der Märkte. 2001.

Band 22 Simone Claber: Großbritannien und die Europäische Integration unter besonderer Berücksichtigung ordnungspolitischer Aspekte. 2002.

Band 23 Silvia Rottenbiller: Essential Facilities als ordnungspolitisches Problem. 2002.

Band 24 Peggy Dreyhaupt von Speicher: Die Regionen Polens, Ungams und der Tschechischen Republik vor dem EU-Beitritt. Interregionale Disparităten, Bestimmungsfaktoren und Lర̋sungsansätze. 2002.

Band 25 Gerhard Rősl: Seigniorage in der EWU. Eine Analyse der Notenbankgewinnentstehung und -verwendung des Eurosystems. 2002.

Band 26 Jöm Quitzau: Die Vergabe der Femsehrechte an der Fußball-Bundesliga. Wohlfahrtsökonomische, wettbewerbspolitische und sportökonomische Aspekte der Zentralvermarktung. 2003.

Band 27 Małgorzata Stankiewicz: Die polnische Telekommunikation vor dem EU-Beitritt. 2003.

Band 28 Sarah Schniewindt: Einführung von Wettbewerb auf der Letzten Meile. Eine ökonomische Analyse von Netzinfrastruktur und Wettbewerbspotential im Teilnehmeranschlussbereich. 2003.

Band 29 Jens Bruderhausen: Zahlungsbilanzkrisen bei begrenzter Devisenmarkteffizienz. Ein kapitalmarkttheoretischer Ansatz. 2004.

Band 30 Philip Jensch: Einkommensteuerreform oder Einkommensteuerersatz? Altemative Ansätze einer Reform der direkten Besteuerung unter besonderer Berücksichtigung steuerpraktischer Gesichtspunkte. 2004.

Band 31 Karsten J. Adamski: Mikroanalyse eines Grundrentenkonzeptes der Alterssicherung. Eine empirische Studie zur Wirkung einer Grundrente auf Basis des Sozioökonomischen Panels. 2004.

www.peterlang.de 